\title{
Advanced DOC-DPF Model to Predict Soot Accumulation and Pressure Drop in Diesel Particulate Filters
}

Alessandro Cozzolini

Follow this and additional works at: https://researchrepository.wvu.edu/etd

\section{Recommended Citation}

Cozzolini, Alessandro, "Advanced DOC-DPF Model to Predict Soot Accumulation and Pressure Drop in Diesel Particulate Filters" (2014). Graduate Theses, Dissertations, and Problem Reports. 5407.

https://researchrepository.wvu.edu/etd/5407

This Dissertation is protected by copyright and/or related rights. It has been brought to you by the The Research Repository @ WVU with permission from the rights-holder(s). You are free to use this Dissertation in any way that is permitted by the copyright and related rights legislation that applies to your use. For other uses you must obtain permission from the rights-holder(s) directly, unless additional rights are indicated by a Creative Commons license in the record and/ or on the work itself. This Dissertation has been accepted for inclusion in WVU Graduate Theses, Dissertations, and Problem Reports collection by an authorized administrator of The Research Repository @ WVU.

For more information, please contact researchrepository@mail.wvu.edu. 


\title{
Advanced DOC-DPF Model to Predict Soot Accumulation and Pressure Drop in Diesel Particulate Filters
}

\author{
by \\ Alessandro Cozzolini \\ Dissertation submitted to the \\ College of Engineering and Mineral Resources \\ at West Virginia University \\ in partial fulfillment of the requirements \\ for the degree of \\ Doctor of Philosophy \\ in \\ Mechanical Engineering \\ Mridul Gautam, Ph.D., Chair \\ Vincenzo Mulone, Ph.D. \\ Andrew Nix, Ph.D. \\ Ettore Pennestri', Ph.D. \\ Gregory Thompson, Ph.D.
}

Department of Mechanical and Aerospace Engineering

Morgantown, West Virginia

2014

Keywords: Diesel Particulate Filter, Emissions, Soot Model

Copyright 2014 Alessandro Cozzolini 


\begin{abstract}
Advanced DOC-DPF Model to Predict Soot Accumulation and Pressure Drop in Diesel Particulate Filters

by

Alessandro Cozzolini

Doctor of Philosphy in Mechanical Engineering

West Virginia University

Mridul Gautam, Ph.D., Chair
\end{abstract}

Diesel Particulate Filters (DPFs) are regarded as the most effective technology to reduce particulate matter (PM) emissions from modern diesel engines. PM mass collected inside the filter needs to be periodically oxidized to clean the porous walls in order to avoid excessive backpressure that could negatively affect engine and turbocharger performances leading to higher fuel consumption. Diesel Oxidation Catalysts (DOCs), on the other hand, represent an effective means to reduce hydrocarbon $(\mathrm{HC})$ emissions and set the proper chemical conditions at the inlet of the DPF to enhance soot oxidation during filter regeneration. Due to the impact that these devices have on tailpipe emissions, monitoring functions aimed at assessing the health of these systems need to be implemented in the engine on-board diagnostics (OBD) system. Further, in-depth understanding of filtration and regeneration mechanisms, together with the ability of predicting actual DPF loading conditions, could play a key role in optimizing regeneration strategies adopted to keep the particulate filter within safe operating conditions. Hence, the use of real-time, yet accurate models is of primary importance to face with advanced control challenges, such as the integration of DOCs and DPFs with the engine or other critical aftertreatment components, or to properly develop model-based OBD monitors.

This study aims at addressing the challenges related with real-time modeling of both DOCs and DPFs with special regard to the calibration of key parameters. At first, the development of a 1-D model for the diesel oxidation catalyst is presented;: two different approaches representing two different trade-offs in terms of model complexity/speed are 
discussed and analyzed. A 1-D model of a DPF is then presented, addressing the coupling of filtration and regeneration over cake, washcoat and wall thicknesses. Moreover, the approach followed to develop the DPF model is innovative as it is directly integrated via analytical functions, thus improving the discretized approach used in similar models. Finally, an innovative model tuning methodology called Virtual Conditioning is presented and applied to generate robust model calibrations.

Numerical results are compared with experimental data gathered at West Virginia University's (WVU) Engine and Emissions Research Laboratory (EERL) using a Mack heavy-duty diesel engine coupled to a Johnson Matthey C-CRT aftertreatment system. The study shows that: a) DOC model is capable of replicating measured outlet emissions concentrations with an accuracy of $15 \%$ and minimal computational requirements over both steady-state and transient operating conditions; b) DPF wall and washcoat layer present different regeneration and collection dynamics, whose behavior is important to capture filter pressure drop and temporal evolution of the collected mass; c) advanced filtration and regeneration process treatment in the wall together with a robust calibration process allow for the use of constant model parameters to replicate combinations of steady-state and transient engine cycles; and d) the model can replicate filter back pressure and mass history of DPFs under subsequent regeneration and loading processes. 


\section{Contents}

$\begin{array}{ll}\text { Abstract } & \text { i }\end{array}$

List of Figures $\quad$ vii

List of Tables $\quad$ x

List of Symbols $\quad$ xi

1 Introduction 1

1.1 Emissions from Diesel Engines . . . . . . . . . . . . . . . 1

1.1.1 Oxides of Nitrogen . . . . . . . . . . . . . . 2

1.1 .2 Particulate Matter . . . . . . . . . . . . . . . 3

1.2 Emission Regulations . . . . . . . . . . . . . . . . . . . . . 4

1.3 Engine and Emissions Control Technologies . . . . . . . . . . . 6

1.3.1 In-Cylinder Emission Reduction Strategies . . . . . . . . . . 6

1.3.2 Diesel Exhaust Aftertreatment . . . . . . . . . . . . . 8

1.4 Aftertreatment Modeling . . . . . . . . . . . . . . . . . . 17

1.4.1 Diesel Oxidation Catalyst . . . . . . . . . . . . . 17

1.4 .2 Diesel Particulate Filter . . . . . . . . . . . . . . . . 21

1.5 Thesis Objective . . . . . . . . . . . . . . . . 23

2 Modeling of Diesel Oxidation Catalyst 25

2.1 Background . . . . . . . . . . . . . . . . 25

2.2 Model Description . . . . . . . . . . . . . . . . . . 28

2.2.1 Conservation of Mass and DOC Pressure Drop . . . . . . . . . . 30

2.2.2 Chemical Reactions \& Kinetic Rates . . . . . . . . . . . . . . 30

2.2.3 Species Conservation . . . . . . . . . . . . . . . . . . . 32

2.2.4 Energy Balance . . . . . . . . . . . . . . . . . 37

2.2.5 Heat and Mass Transfer Coefficients . . . . . . . . . . . . . 38

2.3 Model Implementation: Elemental Cell Approach . . . . . . . . . . . . . 39

2.3.1 Energy Equations . . . . . . . . . . . . . . . . . . . . . 40

2.3.2 Species Conservation . . . . . . . . . . . . . . 43 
3 Modeling of Diesel Particulate Filter $\quad 45$

3.1 General Structure . . . . . . . . . . . . . . . . . . 45

3.2 Wall Sub-model . . . . . . . . . . . . . . . . . . 49

3.2.1 Continuous Filtration Theory . . . . . . . . . . . . . . 49

3.2.2 Partition between wall and cake layer PM mass . . . . . . . . 56

3.2.3 Continuous Wall Regeneration . . . . . . . . . . . . . . 59

3.3 Layer Sub-Model . . . . . . . . . . . . . . . . . . . 61

3.3.1 PM Thermal Oxidation Kinetics: Cake Layer . . . . . . . . . . . 62

3.3.2 PM Catalytic Oxidation Kinetics: Washcoat Layer . . . . . . . . 65

3.3.3 Oxidation of $\mathrm{NO}$ into $\mathrm{NO}_{2}$ in the Washcoat Layer . . . . . . . . 68

3.4 Energy Sub-model . . . . . . . . . . . . . . . 71

3.5 Pressure Drop Sub-model . . . . . . . . . . . . . . . 73

4 Experimental Setup $\quad 76$

4.1 Engine and Aftertreatment System . . . . . . . . . . . . . . . 76

4.2 Instrumentation and Laboratory Setup . . . . . . . . . . . . . . 78

4.2 .1 Test Matrix . . . . . . . . . . . . . . . 80

4.2.2 Experimental Procedure . . . . . . . . . . . . . 82

5 Calibration Methodology $\quad 84$

5.1 DPF Model . . . . . . . . . . . . . . . . . . 84

5.1.1 Virtual Conditioning . . . . . . . . . . . . . . 84

5.1 .2 Model Tuning . . . . . . . . . . . . . . . . . . 88

5.2 DOC Model . . . . . . . . . . . . . . . . . . . . . . 90

6 Results and Discussion $\quad 94$

6.1 DPF Loading Cycle . . . . . . . . . . . . . . . . . . . 94

6.1.1 Engine Performance and Emissions . . . . . . . . . . . . 94

6.1 .2 DOC Model . . . . . . . . . . . . . . . . . . 96

6.1.3 DPF Model . . . . . . . . . . . . . . . . . 102

6.2 Transient Cycle: Federal Testing Procedure (FTP) . . . . . . . . . . . . 110

6.2.1 Engine Performance and Emissions . . . . . . . . . . . . . . . . 110

6.2 .2 DOC Model . . . . . . . . . . . . . . . . . 112

6.2 .3 DPF Model . . . . . . . . . . . . . . . . . . . 118

6.3 DPF Regeneration Cycle . . . . . . . . . . . . . . . . . . 123

6.3.1 Engine Performance and Emissions . . . . . . . . . . . . . 123

6.3 .2 DOC Model . . . . . . . . . . . . . . . . . . 124

6.3.3 DPF Model . . . . . . . . . . . . . . . . . . . . . . . 128

7 Conclusions and Future Work 132 
7.1 Conclusions . . . . . . . . . . . . . . . . . . . . 132

7.2 Future Work . . . . . . . . . . . . . . . . 136

$\begin{array}{ll}\text { References } & 137\end{array}$ 


\section{List of Figures}

1.1 Thermal Formation of Nitric Oxides . . . . . . . . . . . . . . . . 3

1.2 Particulate Matter Morphology . . . . . . . . . . . . . . . . . 4

1.3 Particulate Matter Composition . . . . . . . . . . . . . . . . . 4

1.4 PM Size Distributions . . . . . . . . . . . . . . . . 5

1.5 Pressure Drop Curves for Clean and Loaded DPFs . . . . . . . . . . . . . 11

1.6 Activation Energy for the Catalytic and Non-Catalytic Pathway of a Chemical Reaction. . . . . . . . . . . . . . . . . . . 13

$1.7 \quad \mathrm{NO}_{2} / \mathrm{NO}_{x}$ Equilibrium as Function of Temperature . . . . . . . . . . 16

1.8 Representation of a DOC Channel. . . . . . . . . . . . . . . . . . . 19

2.1 DOC Virtual Elemental Cell . . . . . . . . . . . . . . . . . . . . 40

2.2 Elemental Cells Connection . . . . . . . . . . . . . . . . . . . 40

2.3 Modules for Species Conservation and Energy Balance . . . . . . . . . . 41

2.4 Simulated Substrate Wall Temperature during an FTP Cycle: case $x / L=0.42$

2.5 Simulated Substrate Wall Temperature during an FTP Cycle: case $x / L=$

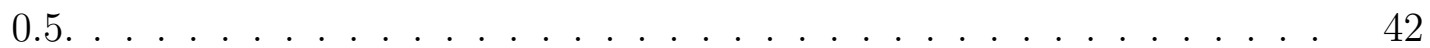

2.6 Simulated Substrate Wall Temperature during an FTP Cycle: case $x / L=1.43$

3.1 Code Structure. . . . . . . . . . . . . . . . . . . . . . . 47

3.2 Representation of the Computational Volume. . . . . . . . . . . . . 48

3.3 Schematic of the Unit Collector Theory . . . . . . . . . . . . . . . 49

3.4 Evolution of Particle Collection Efficiency during a Loading Process. . . . 54

3.5 PM Mass Balance: Partition in Wall and Cake Layer. . . . . . . . . . . . 57

3.6 Graphic Representation of $d w / d x$ in both Washcoat Layer and Substrate Wall. . . . . . . . . . . . . . . . . . . . . 60

3.7 DPF Channel Geometry . . . . . . . . . . . . . . . . . . 74

4.1 Johnson Matthey C-CRT. . . . . . . . . . . . . . . . . . . 77

4.2 Test Engine and C-CRT on Engine Dynamometer at WVU-EERL. . . . . 79

4.3 Laboratory Setup at WVU-EERL. . . . . . . . . . . . . . . 80

4.4 Instrumented C-CRT Used during Engine Testing. . . . . . . . . . . . . . 81

4.5 Pressure drop Measured Across the DPF during the R25 Engine Mode. . 81

5.1 Example of DPF Conditioning. . . . . . . . . . . . . 85 
5.2 Evolution of Substrate Permeability during Model Virtual Conditioning. $\quad 87$

5.3 Evolution of Substrate Properties during Virtual Conditioning. . . . . . . 87

5.4 Distribution of Trapped Mass within the DPF during Virtual Conditioning. 88

5.5 Filter Pressure Drop During Last Part of Virtual Conditioning . . . . . . 88

5.6 Comparison between Measured and Simulated post-DOC Exhaust Temperature. . . . . . . . . . . . . . . . . . . . . . 92

6.1 Time Averaged PM Size Distribution over the R10 Cycle. . . . . . . . . . 95

6.2 Simulated vs Measured Exhaust Temperature: R10 Cycle . . . . . . . . . 97

6.3 DOC Warm-Up during R10 Cycle. . . . . . . . . . . . . . . . . 97

6.4 Continuous Concentrations Downstream the DOC. . . . . . . . . . . 99

6.5 Concentration Distribution over DOC Channel . . . . . . . . . . . . . 100

6.6 Cumulative Mass Emissions Downstream the DOC. . . . . . . . . . . . . 101

6.7 Exhaust Mass Emissions during the DPF Loading Process . . . . . . . . 102

6.8 Overall Pressure Drop during Loading Cycle . . . . . . . . . . . . . . . . 103

6.9 Comparison between Simulated and Measured DPF Pressure Drop during the Loading Cycle 1. . . . . . . . . . . . . . . . . . . . . . 104

6.10 Comparison between Simulated and Measured DPF Pressure Drop during the Loading Cycle 2. . . . . . . . . . . . . . . . . . . . 105

6.11 Comparison between Simulated and Measured DPF Temperature . . . . 106

6.12 Simulated PM Mass Balance within the DPF. . . . . . . . . . . . . . 106

6.13 PM Mass Distribution Within the DPF . . . . . . . . . . . . . . . 107

6.14 PM Mass Distribution Within the DPF . . . . . . . . . . . . . . 108

6.15 Partition of Overall Pressure Drop. . . . . . . . . . . . . . . . . 109

6.16 Experimental versus Simulated PM Loading . . . . . . . . . . . . . . . 109

6.17 Input for DOC and DPF Models during FTP Cycles . . . . . . . . . . . 111

6.18 PM Mass Distribution Within the DPF . . . . . . . . . . . . . . . . . . 112

6.19 Parity Plot for Engine Exhaust Temperature Downstream the DOC: FTP Cycle . . . . . . . . . . . . . . . . . . . . . . 113

6.20 Continuous NO Trace Downstream the DOC: Simulated versus Measured Values. . . . . . . . . . . . . . . . . . . . . . . . . . . 114

6.21 Continuous HC Trace Downstream the DOC: Simulated versus Measured Values. . . . . . . . . . . . . . . . . . . . . . . . . 115

6.22 Continuous CO Trace Downstream the DOC: Simulated versus Measured Values. . . . . . . . . . . . . . . . . . . . . . . . . 115

6.23 Total Mass Emissions over FTP Cycle: Simulated versus Measured Values 116

6.24 Cumulative Mass Emissions over the FTP Cycle . . . . . . . . . . . . . . 117

6.25 Filter Pressure Drop during the FTP Cycle: Comparison between Simulated and Measured Values . . . . . . . . . . . . . . . . . . . . . . . 119 
6.26 Parity Plot for Simulated Filter Pressure Drop . . . . . . . . . . . . . . . 119

6.27 Comparison between DPF Substrate Temperature and Outlet Exhaust Temperature over the FTP Cycle. . . . . . . . . . . . . . . . . . . 120

6.28 Filter Mass Balance over the FTP Cycle . . . . . . . . . . . . . . . . . 121

6.29 Evolution of Soot Layer Thickness during the FTP Cycle. . . . . . . . . 122

6.30 Time Averaged PM Size Distribution over the R100 Cycle. . . . . . . . . 124

6.31 Post-DOC Exhaust Temperature during R100 Cycle: Comparison between Measured and Simulated Results. . . . . . . . . . . . . . . . . . . . . . 125

6.32 Continuous Concentrations Downstream the DOC during the R100 Cycle 126

6.33 Concentration Distribution over DOC Channel Cycle: R100 . . . . . . . 127

6.34 Cumulative Mass Emissions over the R100 Cycle. . . . . . . . . . . . . . 128

6.35 DPF Mass Balance during the R100 Cycle . . . . . . . . . . . . . . . . 129

6.36 DPF Substrate Temperature during R100 Cycle . . . . . . . . . . . . . 129

6.37 Evolution of DPF Properties during Regeneration. . . . . . . . . . . . . 130

6.38 DPF Differential Pressure during R100 Cycle: Comparison between Simulated and Measured Values. . . . . . . . . . . . . . . . . . . 131 


\section{List of Tables}

1.1 EPA Heavy-Duty Diesel Engines Emission Standards . . . . . . . . . 6

2.1 Adsorption Equilibrium Constants . . . . . . . . . . . . . . 33

$3.1 \quad \mathrm{O}_{2}$ and $\mathrm{NO}_{2}$ exponents. . . . . . . . . . . . . . . . . . . . . . . 69

4.1 Test Engine Specifications. . . . . . . . . . . . . . . . . . . 77

4.2 Johnson Matthey C-CRT Specifications. . . . . . . . . . . . . 78

4.3 Characteristics of the Steady-State Engine Modes. . . . . . . . . . . . 82

5.1 DPF Calibrated Parameters . . . . . . . . . . . . . . . . . 90

5.2 Additional DPF Model Parameters. . . . . . . . . . . . . . . . . . . . 90

5.3 DOC Thermal Calibratable Parameters. . . . . . . . . . . . . . . . . . . 92

5.4 DOC Species Conservation Calibratable Parameters. . . . . . . . . . . 93

6.1 Brake Specific Emissions for the R10 Cycle . . . . . . . . . . . . . . . . 94

6.2 Raw Emissions Measured over the R10 cycle. . . . . . . . . . . . . . . . . 95

6.3 Simulated vs Measured Raw Emissions: R10 Cycle . . . . . . . . . . . . 98

6.4 Brake Specific Emissions for the FTP Cycles . . . . . . . . . . . . . . . . 110

6.5 Brake Specific Emissions for the R100 Cycle . . . . . . . . . . . . . . . 123

6.6 Raw Emissions Measured over the R100 cycle. . . . . . . . . . . . . . . . 123

6.7 Simulated vs Measured Raw Emissions: R100 Cycle . . . . . . . . . . . . 125

6.8 Total Mass Emissions Emitted over the R100 Cycle. . . . . . . . . . . . 127 


\section{Notation}

\section{Symbols}

\begin{tabular}{|c|c|c|}
\hline Symbol & Description & Units \\
\hline$A$ & pre-exponential factor in Arrhenius equation & {$[\mathrm{m} / \mathrm{s} / \mathrm{K}]$} \\
\hline$A_{p}$ & average area of soot particles & {$\left[\mathrm{m}^{2}\right]$} \\
\hline$a$ & channel hydraulic diameter & {$[\mathrm{m}]$} \\
\hline$b$ & unit spherical cell size & {$[\mathrm{m}]$} \\
\hline$C_{m}$ & PM mass concentratioon & {$\left[\mathrm{kg} / \mathrm{m}^{3}\right]$} \\
\hline$c_{p g}$ & gas phase specific heat & {$[\mathrm{J} / \mathrm{kg} / \mathrm{K}]$} \\
\hline$c_{p s}$ & solid phase heat capacity & {$[\mathrm{J} / \mathrm{kg} / \mathrm{K}]$} \\
\hline$D$ & diffusion coefficient & {$\left[\mathrm{m}^{2} / \mathrm{s}\right]$} \\
\hline$d_{c}$ & DPF substrate unit collector diameter & {$[\mathrm{m}]$} \\
\hline$d_{\text {pore }}$ & DPF substrate pore diameter & {$[\mathrm{m}]$} \\
\hline$d_{p}$ & PM particle size & {$[\mu \mathrm{m}]$} \\
\hline$E_{i}$ & activation energy & {$[\mathrm{J} / \mathrm{kmol}]$} \\
\hline$E_{\text {cake }}$ & soot layer filtration efficiency & {$[-]$} \\
\hline$F$ & friction coefficient & {$[-]$} \\
\hline$f$ & thermal selectivity & {$[-]$} \\
\hline$G_{i}$ & inhibition factors & {$[\mathrm{K}]$} \\
\hline$g(\epsilon)$ & Kuwabara hydrodynamic factor & {$[-]$} \\
\hline$g_{c} O$ & $\mathrm{CO}$ selectivity of soot oxidation by $\mathrm{NO}_{2}$ & {$[-]$} \\
\hline$\Delta H_{i}$ & heat of reaction & {$[\mathrm{J} / \mathrm{mol}]$} \\
\hline$\Delta H_{a, i}$ & energy factor in adsporption constant equation & {$[\mathrm{J} / \mathrm{mol}]$} \\
\hline$h$ & heat transfer coefficient & {$\left[\mathrm{W} / \mathrm{m}^{2} / \mathrm{K}\right]$} \\
\hline$h_{D}$ & mass transfer coefficient & {$[\mathrm{m} / \mathrm{s}]$} \\
\hline$K_{a, i}$ & adsorption equilibrium constant & {$[-]$} \\
\hline$k$ & DPF wall permeability & {$\left[\mathrm{m}^{2}\right]$} \\
\hline$k_{\text {cat }}$ & rate of catalytic soot oxidation & {$[\mathrm{m} / \mathrm{s}]$} \\
\hline$k_{\text {soot }}$ & soot layer permeability & {$\left[\mathrm{m}^{2}\right]$} \\
\hline$k_{t h}$ & rate of catalytic soot oxidation & {$[\mathrm{m} / \mathrm{s}]$} \\
\hline$L$ & component length (DOC/DPF) & {$[\mathrm{m}]$} \\
\hline
\end{tabular}




$\begin{array}{lll}M_{i} & \text { molecular weigth } & {[\mathrm{kg} / \mathrm{kmol}]} \\ P e & \text { Peclet number } & {[-]} \\ Q & \text { volumetric flow } & {\left[\mathrm{m}^{3} / \mathrm{s}\right]} \\ R & \text { universal gas constant } & {[\mathrm{J} / \mathrm{mol} / \mathrm{K}]} \\ R_{i} & \text { soot layer depletion rate by species "i" } & {[\mathrm{m} / \mathrm{s}]} \\ R a & \text { Rayleigh number } & {[-]} \\ r_{i} & \text { reaction rate } & {\left[\mathrm{mol} / \mathrm{m}^{3} \mathrm{~s}\right]} \\ S_{p} & \text { specific soot area of deposit layer } & {[1 / \mathrm{m}]} \\ u & \text { gas velocity over the longitudinal direction } & {[\mathrm{m} / \mathrm{s}]} \\ u_{w} & \text { interstitial gas velocity } & {[\mathrm{m} / \mathrm{s}]} \\ w & \text { wall thickness } & {[\mathrm{m}]} \\ w_{s} & \text { soot later thickness } & {[\mathrm{m}]} \\ Y_{i} & \text { mole fraction } & {[-]}\end{array}$

\section{Greek Letters}

$\begin{array}{ll}\text { Letter } & \text { Description } \\ \alpha & \text { DPF channel width } \\ \beta & \text { index of completeness of soot oxidaton } \\ \lambda & \text { thermal conductivity } \\ \nu & \text { kinematic viscosity } \\ \epsilon & \text { porosity } \\ \eta & \text { efficiency } \\ \rho & \text { density } \\ \mu & \text { dynamic viscosity } \\ \psi & \text { percolation factor } \\ \phi & \text { partition coefficient }\end{array}$

Units
$\left[\mathrm{m}^{2} / \mathrm{s}\right]$
$[-]$
$[\mathrm{W} / \mathrm{m} / \mathrm{K}]$
$\left[\mathrm{m}^{2} / \mathrm{s}\right]$
$[-]$
$[-]$
$\left[\mathrm{kg} / \mathrm{m}^{3}\right]$
$[\mathrm{kg} / \mathrm{m} / \mathrm{s}]$
$[-]$
$[-]$

\section{Abbreviations}

$\begin{array}{ll}\text { Abbr. } & \text { Description } \\ C R T & \text { continuously regenerating trap } \\ C & \text { carbon } \\ C O & \text { carbon monoxide } \\ C O_{2} & \text { carbon dioxide } \\ C V S & \text { constant volume sampling } \\ D O C & \text { diesel oxidation catalyst } \\ D P F & \text { diesel particulate filter } \\ E C U & \text { engine control unit } \\ E E P S & \text { engine exhaust particle sizer }\end{array}$


EERL engine and emissions reasearch laboratory

EGR exhaust gas recirculation

$E S C \quad$ european stationary cycle

FTP federal testing procedure

$H C$ hydrocarbons

$L A F Y \quad$ Los Angeles freeway

$N O_{x} \quad$ oxides of nitrogen

$\mathrm{O}_{2} \quad$ oxygen

$P M \quad$ particulate matter

$S C R \quad$ selective catalytic reduction

USLD ultra low sulfur diesel

$V G T \quad$ variable geometry turbocharger 


\section{Chapter 1}

\section{Introduction}

Modern diesel engines are traditionally identified as one of the most versatile power sources available for both on-road and off-road automotive applications. The reduced fuel consumption and high torque output coupled with excellent reliability and durability made these engines the best solution for heavy-duty applications. Further, continuous improvements in engine performance have led this technology to be an interesting solution also in regards to light-duty applications due to the higher thermal efficiency which ultimately translates into better fuel economy compared with gasoline engines of same power and lower $\mathrm{CO}_{2}$ emissions for mile traveled [1, 2]. Among the technical reasons which contribute to better performance of modern diesel engines important contribution comes from the ability to utilize a much higher compression ratio and to operate without the need of a throttle, facts that lead to increased thermal efficiency and reduced pumping losses respectively [3].

\subsection{Emissions from Diesel Engines}

Although modern diesel engines offer benefits in terms of fuel economy and performance, the characteristic lean operation still makes control and reduction of diesel exhaust emissions a major engineering challenge. Regulated exhaust emissions from diesel engines include unburned hydrocarbons (HC) and carbon monoxide (CO) as well as oxides of nitrogen $\left(\mathrm{NO}_{x}\right)$ and particulate matter $(\mathrm{PM})$; due to the overall lean combustion 
of diesel fuel, in-cylinder control of $\mathrm{NO}_{x}$ formation is extremely hard to achieve. Further, the heterogeneous nature of the air/fuel mixture at the same time enhances the formation of particulate emissions; it is common to refer to the $\mathrm{NO}_{x} / \mathrm{PM}$ trade-off relation where the factors commonly known to induce a reduction in $\mathrm{NO}_{x}$ formation usually promote the generation of particulate matter and viceversa.

\subsubsection{Oxides of Nitrogen}

Nitric oxide (NO) and nitrogen dioxide $\left(\mathrm{NO}_{2}\right)$ are collectively identified as oxides of nitrogen $\mathrm{NO}_{x}$, with $\mathrm{NO}$ representing the larger portion of the two. The main source of $\mathrm{NO}$ formation is a thermal mechanism known as extended Zeldovitch mechanism [4]. These reactions typically occur in the combustion chamber at temperatures above $1800 \mathrm{~K}$; three main chemical reactions that participate in the Zeldovitch mechanism can be described as follows:

$$
\begin{aligned}
& N_{2}+\mathrm{O} \rightarrow \mathrm{NO}+\mathrm{N} \\
& \mathrm{N}+\mathrm{O}_{2} \rightarrow \mathrm{NO}+\mathrm{O} \\
& \mathrm{N}+\mathrm{OH} \rightarrow \mathrm{NO}+\mathrm{H}
\end{aligned}
$$

The reactions listed above are relatively slow and very sensitive to temperature; nitric oxide emissions produced by this mechanism appear in significant quantities only after the start of heat release, as depicted in Figure 1.1. Conversely, $\mathrm{NO}_{2}$ is formed by recombination reactions between $\mathrm{NO}$ and various other oxidants taking place at engine temperatures below $1200 \mathrm{~K}$, while at higher temperatures it will quickly revert back to $\mathrm{NO}$ in the presence of $\mathrm{O}_{2}$.

One effect of the relationship between combustion temperature and $\mathrm{NO}_{x}$ formation is that any technology or engine control strategy which impacts on the rate of heat release will considerably affect $\mathrm{NO}_{x}$ emissions $[6,7]$; therefore it is not surprising that modern engine controls employ strategies involving simultaneous adjustments of exhaust gas recirculation, start of injection and injection pressure to achieve a reduction of $\mathrm{NO}_{x}$ 


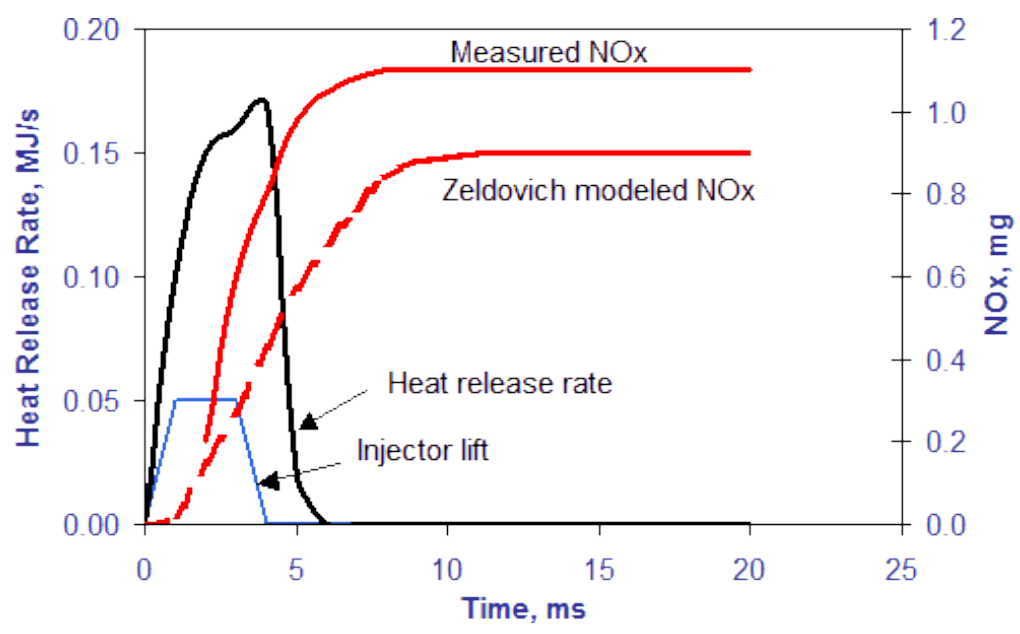

Figure 1.1: Graphic representation of the thermal formation of nitric oxide during the combustion process [5].

emissions $[8,9]$.

Oxides of nitrogen are an object of environmental concerns due the reactivity with volatile organic fraction (VOC) in presence of sunlight to form ground-level ozone $\left(\mathrm{O}_{3}\right)$ [10]. While ozone formed in the stratosphere behaves as a barrier protecting the earth, the ozone at ground level can lead to respiratory illness and other health problems [11-13].

\subsubsection{Particulate Matter}

Diesel particulate matter consists mainly of solid carbonaceous material and ash combined with volatile organic and sulfur compounds into a complex structure, schematically depicted in Figure 1.2 and Figure 1.3. The solid carbonaceous part of PM is formed in fuel-rich regions during the combustion process and subsequently oxidized when excess oxygen is present [14].

However, part of the solid carbon may escape the oxidation process and form a residue of fine carbon cores suspended in the engine exhaust. As engine exhaust starts to cool down passing through the exhaust manifold, carbon cores agglomerate forming particles in the 0.05 to $1.0 \mu \mathrm{m}$ size range. An idealized diesel aerosol number and mass-weighted distributions from [16] is given in Figure 1.4. 

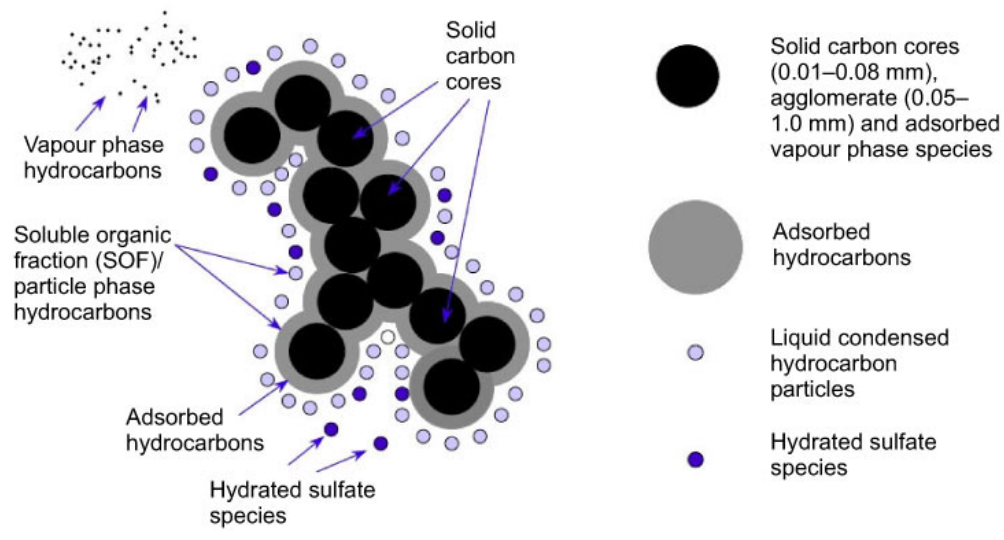

Figure 1.2: Graphic representation of particulate matter morphology [15].

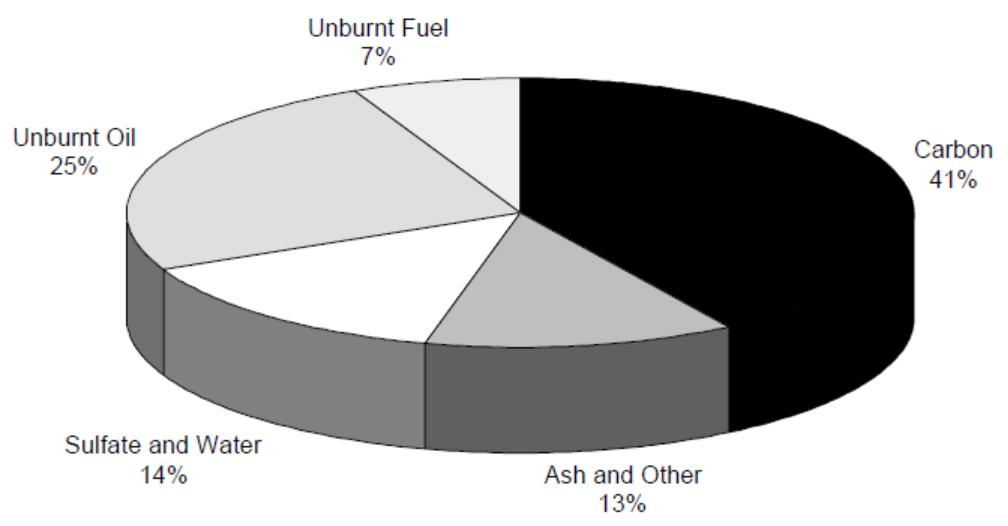

Figure 1.3: General composition of particulate matter [16]

Chemical and morphological characteristics [17] of PM are such that epidemiological [18] and toxicological [19] studies have identified diesel particulate matter as a source for adverse respiratory health effects [20] and potential carcinogen [21].

\subsection{Emission Regulations}

Owing to health and environmental concerns correlated with engine exhaust emissions, the Environmental Protection Agency (EPA) and the California Air Resource Board (CARB) established regulations under the Clean Air Act Amendments (CAAA) in an effort to push manufacturers to limit exhaust emissions from diesel engines used in automotive applications [22]. Similarly, emission regulations have been developed in Europe by the European Parliament and Council.

Emissions limits for US heavy-duty compression ignition engines are listed in Ta- 


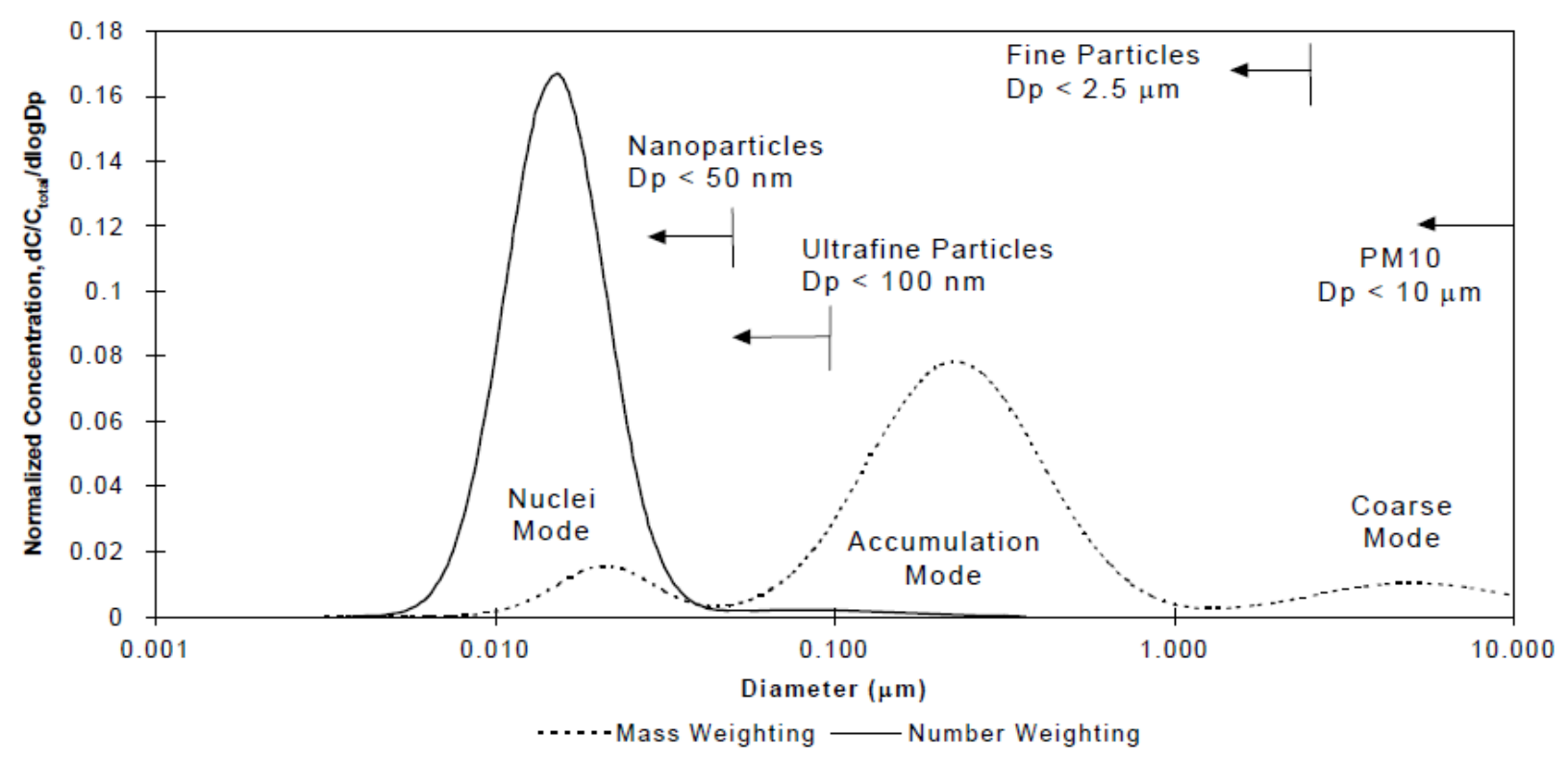

Figure 1.4: Mass and number based particle size distribution [16].

ble 1.1; it is worth noting how $\mathrm{NO}_{x}$ and $\mathrm{PM}$ standards have been reduced by $95 \%$ and 90\% respectively over the past two decades. Further, in an effort to ensure that on-road vehicles remain as clean as possible over the entire useful life, both EPA and CARB introduced regulations to define requirements for an engine on-board diagnostic (OBD) system. These regulations require the development of a monitoring system capable of detecting malfunctions of some key engine subsystems and components whose failure would critically increase tailpipe emissions.

Whenever a malfunction is detected, the OBD system is required to a) illuminate a malfunction indicator light (MIL) to notify the driver of the failure, and b) store a fault code identifying the detected malfunction [23].

The introduction and evolution of emission regulations had a considerable impact on the development of the technology currently employed in modern diesel engines. Developments in heavy-duty engines have been primarily aimed at improving fuel economy, durability, reliability, and costs resulting in advances that tend to be conservative and incremental [24]. However, the introduction of new legislative requirements forced the adoption of new technologies in order to achieve the desired emission levels and, at the same time, guarantee the lowest fuel consumption. 
Table 1.1: EPA Emission Standards [g/bhp-hr] [22]

\begin{tabular}{lcccc}
\hline \hline & & & & \\
Model Year & HC/NMHC & $\mathrm{CO}$ & $\mathrm{NO}_{x}$ & $\mathrm{PM}$ \\
\hline $1979-84$ & 1.5 & 25 & - & - \\
$1985-87$ & 1.3 & 15.5 & 10.7 & - \\
$1988-89$ & 1.3 & 15.5 & 10.7 & 0.6 \\
1990 & 1.3 & 15.5 & 6 & 0.6 \\
$1991-93$ & 1.3 & 15.5 & 5 & 0.25 \\
$1994-97$ & 1.3 & 15.5 & 5 & 0.1 \\
$1998-2003$ & 1.3 & 15.5 & 4 & 0.1 \\
$2004-2006$ & - & 15.5 & - & 0.1 \\
2007 & 0.14 & 15.5 & 0.2 & 0.01 \\
\hline \hline
\end{tabular}

In contrast from light-duty applications where advanced combustion strategies have started to be implemented to optimize mainly low-load engine operating conditions $[25$, 26], developments on heavy-duty diesel engines addressed more traditional strategies and hardware which tend to be more beneficial at higher engine loads [27], conditions typically encountered in heavy-duty applications.

\subsection{Engine and Emissions Control Technologies}

\subsubsection{In-Cylinder Emission Reduction Strategies}

In an effort to answer to the increasingly stringent emission regulations, engine manufacturers introduced several new technologies over the past decade. One of the most effective means to reduce engine-out $\mathrm{NO}_{x}$ emissions is exhaust gas recirculation (EGR). The basic principle behind the EGR system is to recirculate a portion of the exhaust gas back to the intake line. The addition of engine exhaust to intake air increases the overall heat capacity of the charge, leading to a reduction of peak combustion temperature. Since most of $\mathrm{NO}_{x}$ formation can be addressed to the thermal mechanism, a reduction in combustion temperature translates into an abatement of $\mathrm{NO}_{x}$ emissions. However, lower temperature of combustion and reduced oxygen availability typically leads to a decrease in thermal efficiency, worsens fuel economy and increases PM emissions. 
The recirculation of the EGR flow can be achieved in several ways: internally, by means of valve lift overlapping, or externally by means of a pressure differential between exhaust and intake lines. The most common configuration used in modern heavy-duty engines is the Externally Cooled High Pressure Loop EGR. In this particular configuration the EGR flow is redirected into the intake manifold from the exhaust manifold upstream the turbocharger. This approach allows for flexible control of the amount of EGR recirculation as the differential pressure across intake and exhaust manifold may be controlled using intake throttling, variable geometry turbochargers (VGT), and venturi devices. A drawback of this particular implementation is that it leads to a deterioration of fuel economy due to the increased back pressure needed in the exhaust manifold to drive the EGR flow. Recently, new developments in turbocharger technology enabled a recovery of fuel economy when compared to traditional VGT systems [28].

Although EGR systems are considered the most efficient means to successfully reduce engine-out $\mathrm{NO}_{x}$ emissions, research efforts have been redirected on the development of advanced combustion modes aimed at lowering both $\mathrm{NO}_{x}$ and PM [29, 30]. Among all the possible combustion strategies, Premixed Diesel Combustion appears to be the most popular to be proven to achieve simultaneous reduction of both particulate matter and oxides of nitrogen. The basic idea is to lower temperature of combustion to reduce $\mathrm{NO}_{x}$ and, at the same time to reduce the heterogeneity of the air/fuel mixture in an effort to minimize the fuel-rich areas known to be source of PM formation [6].

If it is true that efforts have been dedicated to the development of combustion modes capable of limiting pollutants engine-out levels, it is also true that with the introduction of the US-EPA 2007 and 2010 heavy-duty emission standards the control strategies employed in HDD engines changed considerably due to the adoption of exhaust aftertreatment systems $[31,32]$. 


\subsubsection{Diesel Exhaust Aftertreatment}

The need to meet emission standards and at the same time minimize fuel consumption pushed engine manufacturers towards the adoption of devices used to treat the engine exhaust after it leaves the combustion chamber. These devices are commonly labeled as exhaust aftertreatment systems and represent standard equipment for a heavy-duty vehicle compliant with the most recent US emission standards. In its most general configuration an aftertreatment device consists of a monolith coated with some precious/catalytic material and placed in a steel canister. Depending upon the particular application the aftertreatment system may present different features.

\section{Selective Catalytic Reduction}

The Selective Catalytic Reduction (SCR) of $\mathrm{NO}_{x}$ is the leading technology used to meet the emission limits outlined in the US-2010 HD emission regulation; due to the excess oxygen present in the engine exhaust, reduction of $\mathrm{NO}_{x}$ to $\mathrm{N}_{2}$ and $\mathrm{H}_{2} \mathrm{O}$ is a difficult task $[33,34]$. The reduction can be achieved by passing the engine exhaust through a catalyst in the presence of a reducing agent [35]. The noble metals used to catalyze the reduction can vary from metals of $\mathrm{Pt}[36,37]$ to $\mathrm{Cu}[38]$ and zeolites with metal oxides $[36,39]$.

The reducing agent commonly used in mobile applications is urea at $32.5 \%$ which is injected in the exhaust upstream of the SCR monolith. The injected urea eventually decomposes to give $\mathrm{NH}_{3}$ via the two-step reaction:

$$
\begin{gathered}
\left(\mathrm{NH}_{2}\right) \mathrm{C}(\mathrm{O}) \mathrm{NH}_{2} \rightarrow \mathrm{HNCO}+\mathrm{NH}_{3} \\
\mathrm{HNCO}+\mathrm{H}_{2} \mathrm{O} \rightarrow \mathrm{CO}_{2}+\mathrm{NH}_{3}
\end{gathered}
$$

Over the SCR catalyst, ammonia participates in several chemical reactions which reduce $\mathrm{NO}_{x}$ to elemental nitrogen. The most relevant reactions taking place in the SCR 
substrate are [40]:

$$
\begin{aligned}
& 4 \mathrm{NH}_{3}+4 \mathrm{NO}+\mathrm{O}_{2} ; \rightarrow 4 \mathrm{~N}_{2}+6 \mathrm{H}_{2} \mathrm{O} \\
& 4 \mathrm{NH}_{3}+2 \mathrm{NO}_{2}+\mathrm{O}_{2} ; \rightarrow 3 \mathrm{~N}_{2}+6 \mathrm{H}_{2} \mathrm{O} \\
& 2 \mathrm{NH}_{3}+\mathrm{NO}+\mathrm{NO}_{2} ; \rightarrow 2 \mathrm{~N}_{2}+3 \mathrm{H}_{2} \mathrm{O}
\end{aligned}
$$

The first equation is known as standard $S C R$ reaction where one mole of ammonia reduces one mole of NO. The second equation is addressed as $\mathrm{NO}_{2}-\mathrm{SCR}$ and uses $\mathrm{NO}_{2}$ as main reagent to produce $\mathrm{N}_{2}$ and $\mathrm{H}_{2} 0$. The optimal temperature window for these two reactions falls between $300^{\circ} \mathrm{C}$ and $400^{\circ} \mathrm{C}$ [41]. In this context extensive research work is being conducted to determine the optimal combination of metal catalysts to shift the optimal temperature window towards lower values and thus enhance SCR activities at those engine points characterized by low loads [42, 43]. The third and last reaction is known as fast $S C R$ reaction and it is faster than the standard SCR by one order of magnitude, especially at low temperatures $[44,45]$. It is worth noting that this reaction requires equal parts of $\mathrm{NO}$ and $\mathrm{NO}_{2}$ whilst the engine-out composition of diesel exhaust is approximately $90 \% \mathrm{NO}$ [46]. In order to guarantee such $\mathrm{NO} / \mathrm{NO}_{x}$ ratio $[47,48]$, it is common practice to place a Diesel Oxidation Catalyist (DOC) upstream of the SCR [1] to convert part of the engine-out $\mathrm{NO}$ into $\mathrm{NO}_{2}$.

In order to achieve optimum performances, SCR systems require implementation of advanced control logics in the engine/aftertreatment control units (ECU/ACU) [49, 50]. The aim of these controls is to optimize the injection rates of urea at any given engine load/speed point to maximize $\mathrm{NO}_{x}$ conversion efficiency and, at the same time, limit the possible ammonia slip [51].

Although the implementation and calibration of a SCR system can result in a difficult and resource-consuming task, one of the main advantages offered by the adoption of this technology is represented by the possibility to de-couple tailpipe and engine-out emissions [24]; the high $\mathrm{NO}_{x}$ removal efficiency enables the implementation of engine 
calibrations aimed at maximizing thermal efficiency and fuel economy [32, 7].

\section{Diesel Particulate Filter}

The Diesel Particulate Filter (DPF) is one of the leading and widely used technologies for particulate matter removal in diesel exhaust [52]. Although considerable improvements in engine combustion efficiency have been achieved during the past decade, the amount of PM produced by modern diesel engines requires the adoption of this aftertreatment component to clean the exhaust from solid particles [53]. In its most general configuration, the DPF is formed by a wall-through monolithic device composed of hundreds of adjacent channels that are alternatively blocked at the filter outlet thus forcing the exhaust to flow through the filter wall where PM particles are collected.

The filtration mechanism in a DPF strongly depends on filter material and design [54]. The most common configuration is represented by a wall-through monolith made of a porous ceramic material known as cordierite. This synthetic material is characterized by a low thermal expansion coefficient which increases the mechanical integrity of the filter and a microstructure with average porosity between $45 \%$ and $55 \%$ [55] and a pore size in the range 10 to $20 \mu \mathrm{m}$.

The typical filtration process taking place in a monolithic DPF is a complex phenomenon that depends upon the history of the filter itself; particle collection mechanisms can in fact be totally different between a clean and a loaded/conditioned filter $[56,57]$.

In case of a clean filter, the filtration process initially takes place in the substrate wall and is referred to as deep-bed filtration. During deep-bed filtration particles are collected in the wall pores via three main mechanisms [58]: Brownian diffusion, inertial impaction and interception collection [59]. The relative intensity of these mechanisms depends upon particle size and flow velocity.

As the porous wall becomes less and less porous due to the accumulation of PM, some particles eventually will start to deposit directly on the wall surface leading to the "cake 


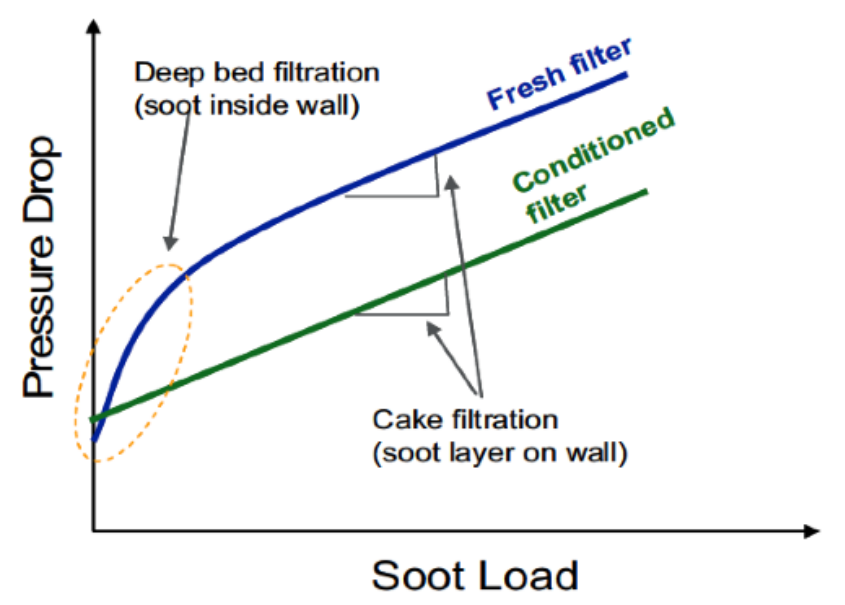

Figure 1.5: Pressure/Soot Load relationships for a clean and conditioned DPF [56].

filtration mechanism. During cake filtration, particles are prevented from entering the wall structure and start to deposit on the surface of the wall leading to a change in the relationship between mass trapped and filter pressure drop (Figure 1.5). The cake layer starts to act as an additional filter on top of the substrate wall and the growth of the layer is directly proportional to the increase in differential pressure across the filter.

Although characterized by different rates, both deep-bed and cake filtration mechanisms lead to increased resistance of the exhaust flow through the soot layer and filter wall resulting in an increase of the overall pressure drop across the DPF and thus higher backpressure acting on the exhaust ports of the engine. In order to reduce the negative effects induced by higher values of backpressure, PM trapped in the DPF needs to be periodically oxidized to clean both wall surface and substrate pores and reduce pressure differential across the filter.

Although considerable amount of oxygen is available in the exhaust due to the lean engine operation, soot oxidation needs considerably high temperatures, of the order of $600^{\circ} \mathrm{C}[60,61]$, to activate; however, heavy-duty diesel engines do not generate exhaust temperatures in this range during normal operation. Hence, a multitude of techniques and strategies have been developed to ensure periodic DPF regeneration. These methods can eventually be categorized in two groups: active and passive methods. 
In active systems, filter regeneration is triggered by manually raising the exhaust temperature to levels that are suitable for soot oxidation. Usually this is achieved with the combustion of additional fuel at pre-determined intervals. Duration and number of regeneration events can be optimized considering integrated fuel consumption, engine time, and vehicle total distance. Nevertheless the past few years have seen also the development of model-based control strategies [62-65] to determine frequency of filter regenerations.

Several approaches exist to raise the exhaust temperature via utilization of diesel fuel: injection of fuel directly into the exhaust line, late in-cylinder injection, throttling [66], and temporary alteration of the engine calibration to obtain higher engine-out temperatures. In case of post injection of diesel fuel, the strategy includes the utilization of a diesel oxidation catalyst (DOC) upstream of the DPF [67] where the injected hydrocarbons are oxidized thus releasing the necessary heat.

Although active systems offer the possibility to control the onset of regeneration and allow for implementation of strategies aimed at optimizing the process, there will always be a fuel penalty associated with this approach. Further, since oxidation of soot is an exothermic process there exists the risk to reach temperatures so high in the filter to produce irreversible damages to the filter substrates (cracks and/or melting).

In an effort to reduce the need for active regeneration events, research has been focused towards the so-called continuously regenerating traps $[53,60]$. This type of PM filter uses the $\mathrm{NO}_{2}$ contained in the engine exhaust to continuously oxidize PM at much lower temperatures $\left(300 / 400^{\circ} \mathrm{C}\right)[55,68]$. For this reason, continuously regenerating traps (CRTs) usually are coupled with a DOC placed upstream the filter to increase the $\mathrm{NO}_{2}$ to- $\mathrm{NO}_{x}$ ratio of the exhaust entering the device. Further, in order to make the continuous oxidation process even more efficient, some applications include the deposition of catalytic coating also on the substrate of the PM filter to lower the activation energy required by the oxidation process. 


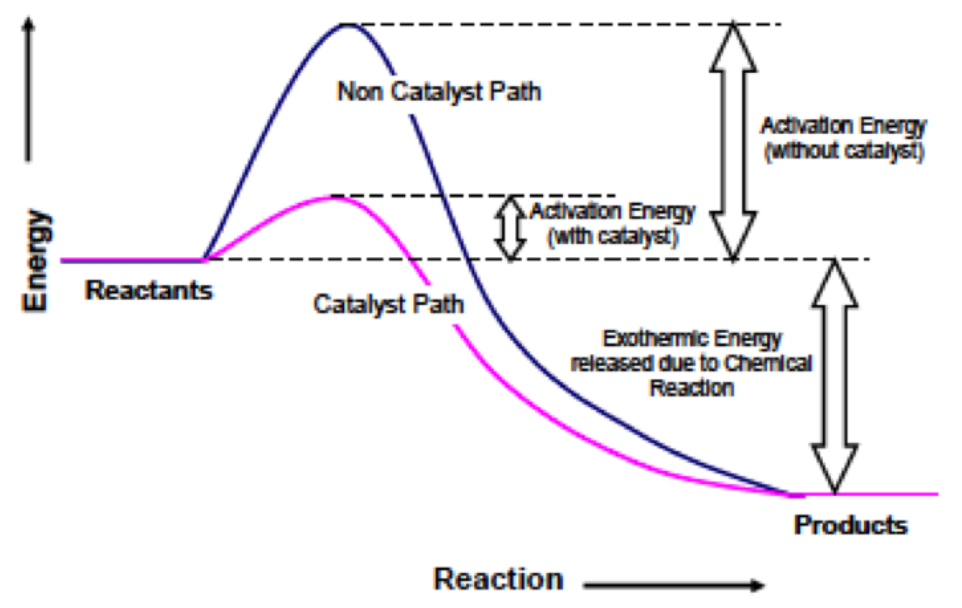

Figure 1.6: Activation energies for catalytic and non-catalytic pathway of a chemical reaction [69].

Such a system is commonly referred to as catalyzed particulate filter (CPF) and usually is more compact than a CRT system. However, the soot layer that builds up on top of the filter walls undergoes a less efficient oxidation in a CPF than in a CRT system. This is due to the fact that in a CPF nitric oxide is converted into $\mathrm{NO}_{2}$ underneath the soot layer whereas in a CRT system $\mathrm{NO}_{2}$ is formed in the DOC upstream the DPF and has therefore a higher chance to make contact with soot particles [55]. In order to combine the advantages of the two technologies, some applications use a DOC upstream of CPF to maximize the use of the $\mathrm{NO}_{x}$ emitted by the engine.

Due to the direct impact that DPF's have on tailpipe PM emissions and due to the possibility of failures to occurr, EPA and CARB mandated the implementation of dedicated monitoring functions in the OBD system. The aim of these monitors is to periodically check the health of the DPF system to ensure compliance with PM emission standards. In particular, depending on the vehicle model year, particulate filters need to be monitored for the following malfunction criteria [23]:

- Filtering Performance: the OBD system is required to detect a decrease in filtration efficiency before PM emissions reach a certain threshold.

- Frequent Regeneration: the monitor function should detect a malfunction when the regeneration of the PM trap occurs more frequently than what is specified by 
the manufacturer leading PM emissions to exceed certain thresholds.

- Incomplete Regeneration: the OBD system should detect a malfunction when the filter does not regenerate properly.

- Missing Substrate: the OBD system should detect a malfunction if either the PM filter substrate is completely destroyed, removed, or missing.

- Active/Intrusive Injection: the OBD system should detect a malfunction if the injection systems ability to regulate fuel injection deteriorates to a point where the PM filter is unable to achieve proper regeneration.

- Feedback Control: the OBD system should detect any failure of the control system that governs filter regeneration.

One of the methodologies used to fulfill these requirements is to use model-based diagnostics where certain measured variables are compared to expected modeled values to deliver a diagnosis of the systems health [70].

\section{Diesel Oxidation Catalyst}

Diesel engine exhaust is a lean mixture usually lower in temperature than the gasoline counterpart. Due to this reason the three-way catalyst (TWC) normally used in stoichiometric gasoline/natural gas applications fails to operate in diesel exhaust. While SCR systems and DPFs are used to limit $\mathrm{NO}_{x}$ and PM emissions respectively, the diesel oxidation catalyst is used as a means to reduce carbon monoxide, unburned hydrocarbons and soluble organic fraction (SOF) emissions [71].

With the introduction of DPFs and SCRs, the diesel oxidation catalyst has become a critical component for the performance of the overall exhaust aftertreatment system [72]. The $\mathrm{NO}_{2}$ generated in the DOC from oxidation of NO is used to: a) improve the performance of downstream SCR systems via enhancement of the fast-SCR reaction rate and b) improve passive regeneration of soot within the particulate filter. Further, oxidation of HC's is used to generate exotherms for active regenerations of DPF filters [72, 60]. 
The most important reactions taking place in the diesel oxidation catalyst are:

$$
\begin{gathered}
C_{n} \mathrm{H}_{m}+(n+m / 4) \mathrm{O}_{2} \rightarrow n \mathrm{CO}_{2}+\mathrm{m} / 2 \mathrm{H}_{2} \mathrm{O} \\
\mathrm{CO}+1 / 2 \mathrm{O}_{2} \rightarrow \mathrm{CO}_{2} \\
\mathrm{NO}+1 / 2 \mathrm{O}_{2} \leftrightarrow \mathrm{NO}_{2} \\
\mathrm{H}_{2}+1 / 2 \mathrm{O}_{2} \rightarrow \mathrm{H}_{2} \mathrm{O}
\end{gathered}
$$

In the DOC, the combination of both chemical equilibrium and the mass transfer equilibrium act together in order to achieve steady-state conditions [69]; reactants diffuse onto the catalyst surface where reactions take place. The products of the reactions are then desorbed back into the gas phase. The diffusion of species from gas to solid phase depends on the exhaust temperature and the difference in concentration existing between the two phases. The conversion rate of a particular species can therefore be considered as the minimum of the chemical reaction rate, and physical bulk mass transfer from gas to solid phase.

In case of $\mathrm{HC}$ and $\mathrm{CO}$ oxidation, chemical kinetics in the solid phase governs the performance of the DOC at low temperatures. When the catalyst surface reaches a certain critical temperature known as "light-off temperature the oxidation reactions take place almost immediately and conversion efficiencies rapidly reach a steady-state condition. Above the temperature of light-off the rates of chemical reaction become faster than the rate of mass transfer and the overall conversion process starts to be limited by the mass transfer of components from the bulk flow to catalyst surface.

In case of $\mathrm{NO}$ oxidation, thermodynamic considerations need to be taken into account. The oxidation of NO is an exothermic process, and as such, the reaction is less thermodynamically favored [73] at higher temperatures. Due to these limitations, the conversion efficiency of $\mathrm{NO}$ oxidation reaches its maximum at temperatures in the range $300 / 380^{\circ} \mathrm{C}$ and then starts to decrease again (Figure 1.7). 


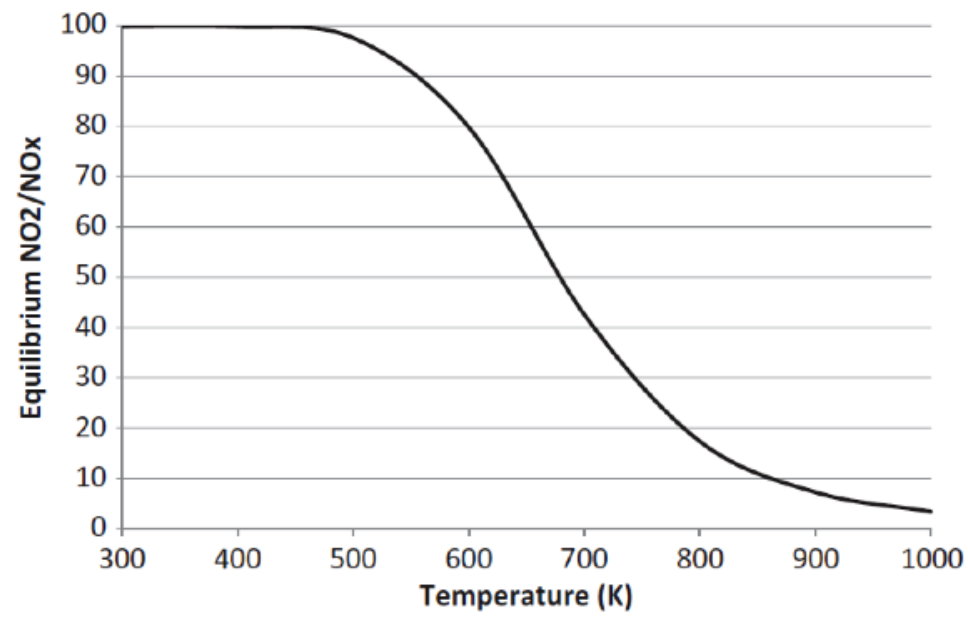

Figure 1.7: $\mathrm{NO}_{2} / \mathrm{NO}_{x}$ Equilibrium as function of temperature [73].

As a result, the oxidation of NO is kinetically limited at low temperatures and thermodynamically limited at higher temperatures.

Conversion efficiencies also strongly depend upon the particular structure of the DOC. As in case of DPFs, also for the case of DOCs ceramic substrates appear to have superior strength when compared to other materials such as metals. Conversely, dispersion of catalyst particles on ceramic substrates is generally more difficult to achieve [74]; for this reason a high surface material is usually applied to the surface of the ceramic substrate (washcoat layer), and the catalyst particles are then dispersed on top of it. Typical washcoat materials include alumina $\left(\mathrm{Al}_{2} \mathrm{O}_{3}\right)$, silica $\left(\mathrm{S}_{i} \mathrm{O}_{2}\right)$ and zeolites with the latter being used for low-temperature applications and to improve performance at cold starts [75-77].

As long as the catalyst material is concerned, platinum $(\mathrm{Pt})$ and palladium $(\mathrm{Pd})$ have been proven to enhance $\mathrm{HC}$ and $\mathrm{CO}$ removal from diesel engines [78, 79] and limit sulfate formation [80] respectively. Finally, the addition of ceria $\left(\mathrm{C}_{e} \mathrm{O}_{2}\right)$ has been proven to lower the light-off temperature [81] thus improving overall performance at lower exhaust temperatures.

Due to the impact on both DPF regeneration and SCR activity, the diesel oxidation catalyst is a component of the aftertreatment system that needs to be periodically monitored to check for possible failures and/or deterioration. The OBD system shall detect 
the following malfunction criteria [23]:

- NMHC Conversion Efficiency: the OBD system is required to detect failure/deterioration of the catalyst that would cause $\mathrm{HC}$ emissions to exceed a certain threshold.

- Aftertreatment Assistance: the OBD system should detect when the DOC is unable to generate a sufficient exotherm to achieve regeneration of the PM filter. Further, the system should be able to detect when the DOC is unable to generate the necessary feedgas constituents for proper SCR system operation.

- Missing Substrate: the OBD system should be able to detect if the DOC is missing.

Similarly to the case of DPFs, a model-based approach is usually adopted to generate monitoring functions capable of fulfilling the requirements listed above.

\subsection{Aftertreatment Modeling}

Although laboratory and on-road testing remain the most effective means to successfully integrate and optimize aftertreatment systems and powertrain, advanced simulation tools provide a promising alternative to the traditional design of experiment. Some of the factor combinations can be analyzed by means of virtual simulations thus minimizing the effective number of experiments and optimizing engine/vehicle testing resources [82]. There exist a plethora of research studies focused on aftertreatment modeling; the modeling approach (from zero-dimensional to tri-dimensional) is usually dictated by type of outcome that the modeling activity is asked to provide.

\subsubsection{Diesel Oxidation Catalyst}

In general, exhaust simulation models owe their complexity to the presence of multiple physical phenomena (mass, heat transfer etc) and chemical reactions which take place 
simultaneously in the DOC $[83,84]$. Although both flow and thermal fields are far from being uniform at the inlet of the monolith, it is common practice to assume that the catalyst is perfectly insulated and that it is exposed to a uniform flow. As a result, the effects of radial thermal gradients are neglected and both temperature and concentration profiles are assumed to be the same in all channels [85]. This allows the DOC to be modeled following the film approach [86] as if it was composed by one single channel; this is a necessary simplification to limit the computational efforts associated with the solution of the governing equations in the whole monolith.

Design variables such as channel size, cell density, wall thickness, and material of the support as well as channel cross-sectional shape play an important role on the overall performance of the converter. In order to correctly replicate those processes and gain knowledge on the relations between design parameters and conversion efficiencies, 3-D models of the DOC channel have been presented in the literature. These models are capable of providing insight in to local parameters such as temperature, velocity, density and pressure drop parameters opposed to spatially-averaged values.

The flow field in the thin channels of a honeycomb structure is usually laminar and it can be modeled by the three-dimensional Navier-Stokes equations which, however, are known to require significant computing efforts. Given the influence of the catalyst support $[87,88]$ and catalyst composition/loading $[53,61]$ on DOC performances, the need for a tool to perform simple case studies involving these design parameters pushed the development of models where detailed descriptions of the three dimensional flow field is coupled to energy conservation and complex chemical kinetics. Cordiner et al. [89], as an example, employed a 3-D model to analyze the effects of washcoat in-channel distribution on light-off temperatures.

Given the axi-symmetric shape of the channels, the model can be simplified analyzing both the transport and kinetic processes in two dimensions. As a consequence, the mass and heat transfer in the single channel can be mathematically described by two 


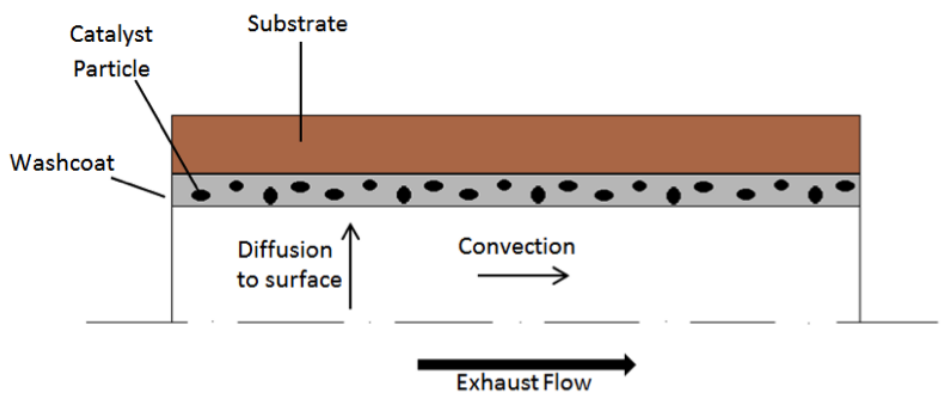

Figure 1.8: Simplified representation of the channel of a diesel oxidation catalyst.

equations: a convection-diffusion equation in the fluid coupled with a species conservation equation in the solid phase. It is worth noting that these models include the 2-D computation of flow and thermal fields in the channel, coupled with species conservation and wall reaction [90]. Given the possibility to impose the flux boundary condition at the fluid-washcoat interface, the results obtained with this modeling approach are usually in accordance with those derived from the more complex 3-D formulation and still offer the possibility to analyze the effects of parameter such as catalyst loading and its axial distribution.

Although these approaches have proven to be an effective means during the design phase, multi-dimensional simulation of a DOC channel still requires strong computational time which would make it nearly impossible to be used for simulation of full transient regulatory cycles. This represents one of the reasons that led to the development and adoption of 1-D two-phase models, obtained by averaging properties and variables over the channel cross-section.

The radial gradients in temperature and concentration between fluid and wall surface collapses in a discontinuity which is accounted for by the introduction of asymptotic heat and mass transfer coefficients based on Nusselt and Sherwood numbers respectively. This approach has proven to be adequate as the intra-pore diffusion taking place in the washcoat is negligible for the wall thicknesses used in aftertreatment components [91]. 
Although these assumptions represent a valuable step towards the simplification of the mathematical representation of the processes taking place in the DOC, real-time solution of a system of non-linear coupled algebraic-differential equations is still a major challenge. On the other side, simpler integral approaches may lack the accuracy needed to correctly replicate thermal and chemical dynamics over transient conditions normally encountered during on-road operation.

As long as the chemical kinetics is concerned, one of the issues associated with asymptotic models lies in having accurate reaction kinetics for all of the reacting species over the specified catalyst formulation [86]. In general, two approaches can be followed to represent reaction kinetics in a converter: micro-kinetic and macro-kinetic approaches. Although the first method allows for more accurate estimation of the reaction rates, it is also true that development of a step-by-step reaction pathway for each of the reactions of interest makes the problem extremely complex and time consuming. Extensive experimental activity involving the usage of synthetic gas benches and small-scale samples of the monolith are necessary to generate those sets of data needed for the calibration of the reaction kinetics model $[92,93]$. Further, the obtained calibration parameters may need adjustments when applied to real-world diesel exhaust [94]. This fact generated motivation in the research community to investigate on the reasons for this discrepancy; it is common agreement that one of the cause for the disparity between synthetic gas bench and real-world exhaust data can be attributed to the speciation of hydrocarbons [95].

On the other hand, the second approach involving the development of global reaction rates offers the possibility for some simplification; step-by-step reaction pathways are replaced by simpler algebraic equations involving global reaction rates and species conservation. Although synthetic gas bench experiments would represent the most rigorous approach for the correct estimation of calibration parameters such as pre-exponential factors and activation energies, the test matrix over which these rate expressions are calibrated should be representative of the conditions at which the DOC is actually used [96, 97]. A calibration procedure based on data generated using real diesel exhaust and standard-size 
monoliths, however, may be difficult due to the simultaneous effects of reaction kinetics and mass transfer.

\subsubsection{Diesel Particulate Filter}

Similarly to what was presented in the case of the DOC, the increasing interest in DPF functionalities and the need for advanced controls pushed towards the development of a number of computational models characterized by different degrees of detail. Research on DPF modeling can be divided in several categories depending upon the particular object of the study.

While the uniformity of the flow conditions at the inlet of a system [98] is one of the common assumptions used in aftertreatment modeling, the possibility of radial nonuniformities justified the development of macroscopic scale models of DPFs aimed at capturing radial variations of the exhaust gas stream velocity and temperature at the inlet of the filter. This can be attained by either solving the full 3-D flow and thermal fields at the inlet of the DPF [99], or approximating the radial non-uniformities in collections of several channels along the radius of the filter [100], or using a self-consistent continuum model for the entire porous filter following the principles of volume averaging in multiphase flows [101].

In these models, evolution of filter characteristics such as porosity and permeability can be implemented based on integral assumptions extended to the whole filter [102-104]. Further application of 3-D DPF modeling has been presented by Konstandopolous [105] to investigate on the relative contributions of secondary inertial pressure drop terms arising in case of high filtration velocities through the filter walls.

A reduction in dimensionality of the problem is possible with a 2-D approach; when the transient operation is slow compared to the residence time of the exhaust gas within the filter, the quasi-steady state approximation can be used to model the filter channels. When the conditions of the exhaust entering the filter can be assumed to be spatially 
uniform, simulation of a pair of inlet/outlet channels is equivalent to model the whole monolith. Similarly to what was stated for the DOC, this approach is usually preferred due to the proven accuracy and the intrinsic simplicity when compared to the full 3-D approach [106, 107].

A plethora of studies focusing on the development of 2-D models of DPF channels are available in the literature; the basic framework developed by Bisset [108] for the thermal oxidation of soot particles in PM traps coupled to the innovative work of Lee [109] laid the foundations for a prolific field of research focused on DPF modeling. Following studies targeted the adaptation of advanced filtration theories such as those presented by Oh [110] to the wall-flow extruded structures typical of DPFs $[111,58]$ as well as investigation on soot microstructure [112] and its effect on regeneration/filtration behavior. Articles presenting validation of 2-D models against experimental data represent a good portion of the available literature; the development of these tools enabled further analysis of DPF functionalities and control, especially in terms of advanced regeneration strategies $[113$, 114] and analysis of aging [115].

Although two dimensional models have proven to be an effective means to simulate the thermal and chemical processes taking place within a pair of channels, an increasing number of efforts have been directed also towards the development of simpler approaches; this is the case of $1-\mathrm{D}$ and $0-\mathrm{D}$ representations of DPFs.

The 1-D approach is based on the assumption that key phenomena can be integrated over the channel length, direction where changes in variables such as temperature, oxygen and $\mathrm{NO} / \mathrm{NO}_{2}$ concentrations are assumed to remain almost constant. Although many studies presented above have shown how particulate and ash accumulation in DPF channels are not uniform over the longitudinal direction, these spatially lumped models have only temporal dependence and no spatial dependence. Since partial differential equations are replaced with ordinary differential equations, computational time is usually reduced. 
The mass of PM trapped in the filter is assumed to be independent of the radial or axial position and it is allowed to vary only over the filter wall thickness. Also, the mass of particulate collected as a cake layer on top of the substrate wall is assumed to be uniformly distributed over the channel length. Similarly, the temperature of the filter wall is computed considering the filter as a single control volume. The considerable amount of models that have been developed is the result of the proven efficiency of the 1-D approach; detailed temporal evolution of soot load inside the filter, pressure drop and substrate temperature is obtained with a relatively simple set of equations; this fact has enabled the adoption of simple DPF models for development of control strategies [116].

Although model-based theories have been successfully proposed during the past decade, coupled 1-D DOC-DPF models available in the literature are usually calibrated and tested against steady-state data to prove the accuracy of the model to capture filter loading and regeneration. These calibrations are usually engine-mode dependent meaning that a dedicated calibration is employed to match experimental data at each engine operating point. Therefore, the application of these models for diagnostics purposes still represents a major challenge. Nevertheless, with the implementation of the latest OBD requirements model-based diagnostics can be expected to undergo further development; in this regard, simple but accurate modeling of aftertreatment systems is mandatory if effective monitoring of these devices has to be achieved using the limited computational power of an engine/aftertreatment control unit (ECU/ACU).

\subsection{Thesis Objective}

Based on the given background, it is possible to acknowledge the need for further contribution towards the development of a) simple approaches to aftertreatment modeling and b) robust model calibrations based on engine testing data. The achievement of these goals will eventually enable the release of more robust OBD functions to monitor the health of engine/aftertreatment over the vehicle useful life. 
The global objective of this work is to replicate the dynamics taking place in a continuously regenerating PM trap during steady-state and transient operating conditions, using an approach based upon 1-D modeling of the DOC-DPF system. The accomplishment of this primary objective is achieved by completion of the following specific goals:

(a) To improve the state of the art filtration/regeneration theory by application of a continuous analytical treatment for the solution of the governing equations, with special regard to the susbstrate wall. This novel solution method allows for detailed representation of the dynamics taking place in the wall eliminating the need for any type of discretization.

(b) To develop a 1-D DOC model to provide feed-signals for the DPF model in terms of exhaust composition and temperature. The capability of the DPF model to track filter soot loading during transient operations can be achieved provided the input satisfy certain levels of accuracy. Models available in the literature use off-line maps based on steady-state experiments to estimate transient values. This approach leads to significant deviations of the simulated results from measured values, especially during highly transient conditions.

(c) To develop a tuning procedure for the DPF model to generate robust calibrations which are independent of the particular operating condition. This is a novel characteristic as it is common procedure to adjust calibration parameters according to the specific engine mode that needs to be replicated. 


\section{Chapter 2}

\section{Modeling of Diesel Oxidation \\ Catalyst}

This chapter discusses the methodology that was followed to model the diesel oxidation catalyst and the particulate filter. After a review of the most relevant literature is presented, governing equations for species conservation, energy balance and mass balance are discussed in details. Finally, the implementation of the governing equations in Matlab-Simulink is presented.

\subsection{Background}

In order to reproduce the phenomena occurring within a diesel oxidation catalyst, correct modeling of the governing equations is required. To that aim, the set of equations that are commonly employed include energy balances of both gas and solid phase, mass conservation, and species conservation equations. Many different approaches have been proposed to correctly solve this set of equations and different simplifications and hypotheses can be adopted in order to reduce the complexity of the problem.

The most relevant DOC models available in the literature are eventually based on the framework first developed by Voltz et al. [117]. In this work, experimental investigations on the kinetics of $\mathrm{CO}$ and propylene oxidation on a platinum-based catalyst were 
performed. In particular, both Eley-Rideal and Langmuir-Hinshelwood kinetics were discussed and rate equations for $\mathrm{CO}$ and propylene oxidation were generated, which included terms to model the inhibition effects due to $\mathrm{CO}, \mathrm{C}_{3} \mathrm{H}_{6}$ and NO. These kinetic models were at first calibrated against experimental data gathered using synthetic gas mixtures and then extended to the case of real engine exhaust. The calibration process included the definition of a cost objective function and its minimization as means to optimize the calibration parameters.

Cavendish et al. [118] presented a solution based on the Galerkins method for the non-linear transient two-point boundary value problem, which describes the interaction between diffusion and chemical reaction in a catalyst pellet. The methodology was later adopted by $\mathrm{Oh}$ et al. [119] to solve the set of equations of a 1-D mathematical model developed to study the transient response of a DOC to a step change in feedstream temperature as a function of catalyst design parameters such as length and cell density. The equations for the reaction rates that were presented in Voltz et al. [117] were fitted to new reactor data presented in [120].

In a study aimed at evaluating the $\mathrm{NO}_{2}$ formation downstream of a diesel oxidation catalyst Triana et al. [121] proposed a 1-D model for the DOC of a continuously regenerating trap (CRT). The model neglected heat and mass accumulation in the gas phase and assumed quasi steady-state operation due the short residence time of the exhaust in the monolith. Model parameters such as activation energies and pre-exponential factors were calibrated against experimental data gathered running a medium-duty diesel engine at several steady-state operating points covering a wide range of exhaust temperatures and flow rates. The estimated parameters were in accordance with values reported in the literature [118].

In an effort to investigate the performance of catalyzed particulate filters, Kandylas et al. [122] and Koltsakis et al. [123] developed a 1-D model of DOC aimed at simulating the conversion of engine-out $\mathrm{NO}$ into $\mathrm{NO}_{2}$. The objective of the study was to assess the 
impact of a diesel oxidation catalyst placed upstream of a continuously regenerating trap. Apart from converting $\mathrm{CO}$ and $\mathrm{HC}$, the main role of the $\mathrm{DOC}$ is to oxidize $\mathrm{NO}$ into $\mathrm{NO}_{2}$ to enhance the passive oxidation of particulate matter. One of the key features of this study was the introduction of mathematical factors calibrated to model the limiting effects of chemical equilibrium. Further, Koltsakis et al. [123] included analysis of regeneration efficiency via application of a 3-D DPF model.

Further analysis of the dynamics taking place in a continuously regenerating trap is offered by York et al. [124] who presented a 1-D DOC-DPF model calibrated towards micro-reactor data and then validated against engine test data. The DOC model included speciation of hydrocarbons into six components and thermodynamic limitation of the $\mathrm{NO} / \mathrm{NO}_{2}$ chemical reaction. Engine test data included a custom low temperature cycle and the European Stationary Cycle (ESC). The integrated DOC-DPF model was then used to predict the soot load and the balance point for different DOC/DPF configurations.

A general investigation on the difficulties associated with the calibration of DOCs models was provided by Lafossas et al. [92]. The work investigated on the plausibility of transferring the model calibration obtained using small scale catalyst and test bench data to the case of full-scale systems and real exhaust. The outcome of the study is a methodology to use the parameters calibrated over synthetic gas test data to simulate driving cycle conditions with real exhaust without the need of modifications.

Chatterjee et al. [40] presented a model combining a spatially heterogeneous approach for mass and heat balance with a global kinetic approach to model the chemical reactions. The model employed transient mass balance for both gas and solid phases, as well as characterization of washcoat storage capacity. Further, a submodel for the condensation/evaporation of $\mathrm{H}_{2} \mathrm{O}$ was included in the thermal balance to fit simulation results towards experimental data gathered on a FTP cycle. Simulated transient traces of NO and $\mathrm{NO}_{x}$ over the FTP cycle showed agreement with experimental data. 
The dynamics taking place within a diesel oxidation catalyst include both reaction kinetics and convection-diffusion of components from the gas phase to the washcoat; a study that analyzes the mass transfer regime in automotive catalytic converters was proposed by Santos et al. [85] who evaluated the performance of existing correlations for the mass transfer rate expressed as function of thr Sherwood number. The presented data showed that the calculated Sherwood numbers are usually below the asymptotic values and chemical species dependent, hence suggesting the adoption of dedicated expressions for each of the components of engine exhaust.

The model proposed in this work has been developed to accomplish the simultaneous tasks of a) being able to compute conversion efficiencies over transient cycles and b) limit the complexity of the mathematical framework to allow for fast solution of the governing equations. In order to achieve these goals the problem was divided into two separate phases. In the first phase, a classic 1-D model of a DOC was developed using an approach based on previous works and available in the literature. Once a reliable model was obtained, the main parameters were calibrated towards experimental data following the methodology discussed later in this work. When satisfactory agreement between simulated and measured data was achieved, the simplification process was started and a simpler version of the DOC model was developed and tested maintaining the same calibration.

\subsection{Model Description}

This section discusses the mathematical representation of mass and heat transfer as well as chemical kinetics taking place in one channel of a diesel oxidation catalyst. Assuming uniform distribution of variables at the inlet section, identical conditions are supposed to prevail in the channels of the monolith and therefore the representation of the whole reactor can be reduced to the analysis of a single channel.

The assumptions used to derive the governing equations are listed as follows: 
- The DOC operates under quasi steady-state conditions. This assumption is justified by the short residence time of the exhaust gases flowing through the monolith [121].

- Temperature, concentrations, and velocity of the gas phase flowing in a single channel are considered to be cross-sectional averaged quantities. The discontinuity between gas phase and solid phase is accounted for by the introduction of heat and mass transfer coefficients. This assumption allows modeling of the most important phenomena in only one direction thus limiting the complexity of the model itself [85].

- Energy and species accumulation in the gas phase are neglected. This assumption allows neglecting the time derivative term contained in the equations for energy and species conservation of the gas phase [121].

- Axial heat conduction in the gas phase is negligible compared to heat convection.

- Chemical reactions occur at the catalyst surface and the rate of reaction is a function of the substrate temperature. Reactions are assumed to be of the second order and reaction rates constants are assumed to follow the Arrhenius form.

- The substrate temperature is assumed to be constant in the radial direction but it is allowed to vary over the DOC length.

- The exhaust gas is assumed to follow ideal gas behavior.

- The flow within the DOC channel is laminar (low Re numbers) and fully developed [125].

- The compressibility of exhaust gas is not considered due to the low pressure at which DOCs usually operate. 


\subsubsection{Conservation of Mass and DOC Pressure Drop}

The conservation of mass within each channel of a DOC can be expressed as:

$$
\frac{\partial u}{\partial x}+\frac{\partial v}{\partial y}+\frac{\partial w}{\partial z}=0
$$

where $u$, denotes the velocity component in longitudinal direction while, $v$ and $w$ represent the velocity components in the transverse directions. Considering the assumptions listed above, however, equation 2.1 can be simplified to:

$$
\frac{\partial u}{\partial x}=0
$$

The pressure drop across the diesel oxidation catalyst can be modeled using the mass balance for laminar flow in the individual channel [121]:

$$
\Delta P=\frac{2 \mu K L Q}{C F A \text { a OFA }}
$$

where $\mu$ is the dynamic viscosity of the exhaust gas, $K$ is the fanning friction factor [121], $L$ is the length of the channel, $Q$ is the volumetric flow rate, $C F A$ is the frontal area of the monolith, $O F A$ is the open frontal area and, $a$ is the channel hydraulic diameter.

\subsubsection{Chemical Reactions \& Kinetic Rates}

Chemical reaction pathways occurring in a diesel oxidation catalyst can be complicated. Typically, a considerable amount of intermediate reactions can take place simultaneously; also, when considering a limited number of reactions, the problem might remain nontrivial if a multi-step microkinetic model is developed for the oxidation process of the reacting species [126]. Considering the scope of the model being presented in this work, three 1-step chemical reactions involving the pollutants of interest are assumed to take place in the diesel oxidation catalyst:

$$
R_{1}: \mathrm{CO}+\frac{1}{2} \mathrm{O}_{2} \rightarrow \mathrm{CO}_{2}
$$




$$
\begin{aligned}
R_{2}: C_{x} H_{y}+\left(x+\frac{y}{4}\right) O_{2} & \rightarrow x \mathrm{CO}_{2}+\frac{y}{2} \mathrm{H}_{2} \mathrm{O} \\
R_{3}: N O+\frac{1}{2} \mathrm{O}_{2} & \leftrightarrow \mathrm{NO}_{2}
\end{aligned}
$$

Oxidation of carbon monoxide into carbon dioxide is simplified [121] when compared to the rigorous representation presented in $[117,73]$. The oxidation of unburned hydrocarbons can be modeled using equation 2.5 where propane $\left(\mathrm{C}_{3} \mathrm{H}_{6}\right)$ has been selected as representative hydrocarbon in the model although it is not necessarily the most representative hydrocarbon in diesel exhaust. This approach is commonly applied in the literature to model oxidation of hydrocarbons in DOC and it is justified by the relatively low $\mathrm{HC}$ concentrations $(\sim 100 / 200 \mathrm{ppmC})$ in diesel exhaust [121].

The kinetic rate expressions for the chemical reactions listed above are adopted from the literature. In particular, the expressions suggested by Voltz et al. [117] and Oh et al. [119] are used for the reaction rates of equations 2.4 and 2.5, while the formulation proposed by Koltsakis et al. [123] is employed for the reaction rates of equation 2.6:

$$
\begin{gathered}
r_{C O}=\frac{k_{C O} y_{s, C O} y_{s, O_{2}}}{G_{1}} \\
r_{C_{3} H_{6}}=\frac{k_{C_{3} H_{6}} y_{s, C_{3} H_{6}} y_{s, O_{2}}}{G_{2}} \\
r_{N O \rightarrow N O_{2}}=\frac{k_{N O} y_{s, N O} y_{s, O_{2}}}{G_{3}}\left(1-\frac{y_{N O_{2}}}{y_{O_{2}}^{0.5} y_{N O} K_{E q}}\right) \\
r_{N O_{2} \rightarrow N O}=\frac{k_{N O_{2}} y_{s, N O_{2}}}{G_{3}}\left(1-\frac{y_{N O} y_{O_{2}}^{0.5}}{y_{N O_{2}} K_{E q}}\right)
\end{gathered}
$$

It is worth noting that the last two equations vanish when $\mathrm{NO}$ and $\mathrm{NO}_{2}$ reach equilibrium concentrations. In this regard, the expression for the equilibrium constant is adopted from York et al. [124] and is as follows:

$$
\ln \left(K_{e}\right)=7.0325 \frac{1000}{T}-9.078
$$

where the temperature, $T$, has units of $[K]$. 
The reaction rate constants are assumed to follow an Arrhenius form of the type:

$$
k_{i}=A_{i} \exp \left(\frac{-E_{i}}{R T_{s}}\right)
$$

The terms of the form $G_{i}$ appearing in the equations for the rates of chemical reaction account for inhibition effects and can be expressed according to [123, 127] as:

$$
\begin{gathered}
G_{1}=T_{s}\left(1+K_{a, 1} Y_{s, C O}+K a, 2 Y_{s, H C}\right)^{2}\left(1+K_{a, 3} Y_{s, C O}^{2} Y_{s, H C}^{2}\right)\left(1+K_{a, 4} Y_{s, N O}^{0.7}\right) \\
G_{2}=T_{s}\left(1+K_{a, 5} Y_{s, C O}+K a, 6 Y_{s, H C}\right)^{2}\left(1+K_{a, 7} Y_{s, C O}^{2} Y_{s, H C}^{2}\right)\left(1+K_{a, 8} Y_{s, N O}^{0.7}\right)\left(1+K_{a, 9} Y_{s, O_{2}}\right)^{1.5} \\
G_{3}=T_{s}\left(1+K_{a, 10} Y_{s, C O}+K a, 11 Y_{s, H C}\right)^{2}\left(1+K_{a, 12} Y_{s, C O}^{2} Y_{s, H C}^{2}\right)\left(1+K_{a, 13} Y_{s, N O}^{0.7}\right)
\end{gathered}
$$

where the values for the adsorption equilibrium constants can be computed using the following expression [123]:

$$
K_{a, i}=K_{a 0, i} \exp \left(-\frac{\Delta H_{a, i}}{R T_{s}}\right)
$$

The consumption rate of oxygen can be calculated applying an oxygen balance to the chemical reactions previously described to obtain:

$$
r_{O_{2}}=0.5 r_{C O}+4.5 r_{C_{3} H_{6}}+0.5 r_{N O}
$$

\subsubsection{Species Conservation}

Assuming a nigligible rate of intra-pore diffusion in the channel walls [86], the performance of a diesel oxidation catalyst depends mainly on two physiochemical processes that occurr simultaneously as exhaust gas flows through the channel. On one side, there are the rates of chemical reactions taking place at the washcoat surface; on the opposite side, there is the rate of mass transfer of the reacting species from the bulk flow to the 
Table 2.1: Adsorption Equilibrium Constants [123]

\begin{tabular}{lcc}
\hline \hline & & \\
Constant & $\Delta H_{a, i}[\mathrm{~J} / \mathrm{mol}]$ & $K_{a 0, i}$ \\
\hline$K_{a, 1}$ & -7990 & 655 \\
$K_{a, 2}$ & $-310^{5}$ & $2.0810^{3}$ \\
$K_{a, 3}$ & -96534 & 3.98 \\
$K_{a, 4}$ & 31036 & $4.7910^{5}$ \\
$K_{a, 5}$ & -7990 & 655 \\
$K_{a, 6}$ & -3000 & $2.0810^{3}$ \\
$K_{a, 7}$ & -96534 & 3.98 \\
$K_{a, 8}$ & 31036 & $4.7910^{5}$ \\
$K_{a, 9}$ & 0 & 0 \\
$K_{a, 10}$ & 20 & -7990 \\
$K_{a, 11}$ & 100 & -3000 \\
$K_{a, 12}$ & 3.98 & -96534 \\
$K_{a, 13}$ & $210^{5}$ & 31036 \\
\hline \hline
\end{tabular}

channel walls. At steady-state conditions and for temperatures higher than the light-off temperature, a non-uniform transverse concentration of the species is present in a cross section of the channel with the bulk concentration of a reacting species decreasing almost to zero in proximity of the channel wall. At the same time, this difference in concentrations generates and maintains a diffusion flux of reacting species from the bulk phase to the washcoat, thus feeding the chemical reactions.

These simultaneous phenomena are usually modeled by two coupled species conservation equations: one for the gas phase and one for the solid phase. The simultaneous solution of this set of equations ensure the correct representation of the physiochemical processes occurring in the diesel oxidation catalyst.

In this study two different approaches to solve this problem will be presented. The first method, used in the "complete model" in this work, solves for both gas and solid phase species conservation equations according to the procedures commonly discussed in the literature. The second method, used in the "simple model" in this work, drops the solution of the species conservation equation in the solid phase and replaces the 
estimation of the mass transfer-chemical reaction equilibrium with solutions derived from two limiting cases.

\section{Complete Model}

The 1-D equation for the species conservation in gas phase for one channel of diesel oxidation catalyst can be expressed as:

$$
\frac{\partial Y_{g, i}}{\partial t}+u \frac{\partial Y_{g, i}}{\partial x}+h_{D_{i}} S\left(Y_{g, i}-Y_{s, i}\right)=0
$$

while for the solid phase the rate of accumulation of species $i$ in the solid phase equals the difference between the rate of mass transfer to the reacting wall and the rate of chemical reaction according to:

$$
\frac{\partial Y_{s, i}}{\partial t}=\frac{P}{R T_{g}} h_{D_{i}}\left(Y_{g, i}-Y_{s, i}\right)-r_{i}
$$

In the equations presented above the subscripts $g$ and $s$ denote gas and solid phase respectively whilst subscript $i$ refers to the specific gas component $\left(\mathrm{CO}, \mathrm{C}_{3} \mathrm{H}_{6}, \mathrm{NO}\right.$ and $\left.\mathrm{NO}_{2}\right)$. Also, $h_{D_{i}}$ denotes the mass transfer coefficient while, $S$, represents the ratio between the active area and volume of the diesel oxidation catalyst. Further, assuming quasi steady-state operation in light of the short residence time of the exhaust gasses within the channels of the DOC, the equations described above simplify to:

$$
\begin{gathered}
u \frac{\partial Y_{g, i}}{\partial x}=h_{D_{i}} S\left(Y_{s, i}-Y_{g, i}\right) \\
\frac{P}{R T_{g}} h_{D_{i}}\left(Y_{g, i}-Y_{s, i}\right)=r_{i}
\end{gathered}
$$

where it is worth noting that since no accumulation of mass is allowed in the solid phase, the rate of transport of each reacting species from the gas phase to the wall must be equal to the rate of chemical reaction involving the same species. Hence, the behavior of a single channel can be modeled by a convection-diffusion equation for the fluid phase coupled with a reaction equation within the washcoat. Also, Equation 2.20 denotes a set 
of non-linear partial differential equations while Equation 2.21 represents a set of nonlinear, algebraic equations which need to be solved simultaneously. Boundary condition for Equation 2.20 is as follows:

$$
Y_{g, i}(x=0, t)=Y_{g, i}^{\text {inlet }}
$$

\section{Simple Model}

Although rigorous solution of the two sets of equations presented in the previous section would allow for accurate representation of the equilibrium between mass transfer and chemical kinetics, a simpler approach aimed at limiting the complexity of the solution can be defined based on two limiting cases. As discussed earlier in this work, the conversion of pollutants in a diesel oxidation catalyst can be described by two inherently coupled processes: the mass transfer of reacting species from the bulk phase to the substrate and the chemical reaction taking place on the surface on the substrate. This ultimately translates into the need of solving for the surface concentration of the reacting species at every time step. Considerations can be drawn, however, depending on the particular operating condition.

Following an approach already presented in a previous work [63], the two sets of equations presented in the previous section can be dropped and replaced with the following simplified forms:

- For substrate temperatures that are lower than the light-off temperature, the rates of chemical reactions taking place at the surface of the washcoat can be assumed to be negligible; as a result, the concentration of reacting species in the vicinity of the substrate $\left(Y_{s, i}\right)$ remains close to the bulk concentration $\left(Y_{g, i}\right)$ therefore limiting the mass transfer from gas to solid phase. Since the limitation is introduced by the low reaction rate this operating condition is usually referred to as kinetic-limited regime. When the behavior of the diesel oxidation catalyst is kinetic-limited, the rate of chemical reaction is the "dominating" factor and therefore the process is 
represented by the following equations:

$$
\begin{gathered}
Y_{s, i} \simeq Y_{g, i} \\
u \frac{\partial Y_{g, i}}{\partial x}=-r_{i}
\end{gathered}
$$

- For substrate temperatures that are higher than the temperature of light-off, the chemical reactions takes place almost instantaneously on the substrate surface; in these conditions the catalyst performanace is strongly influenced by the transport mechanims of reacting species to the substrate wall. Eventually the mass transfer process becomes rate-limiting at the highest temperatures [128]. In these operating conditions, the rates of chemical reaction are so fast that the concentration of the reacting species in the proximity of the substrate wall drops to values close to zero; as a result the difference in concentration between gas phase and solid phase increases thus enhancing the transport of mass from gas phase to solid phase. Due to the exponential relationship between reaction rate and temperature, however, the rate of chemical reaction becomes much faster than the rate of mass transport. In this case the limitation is introduced by the transport of species from bulk flow to reacting site; this operating condition is usually referred to as transport-limited regime. The behavior of the diesel oxidation catalyst is governed by the following equations:

$$
\begin{gathered}
Y_{s, i} \simeq 0 \\
u \frac{\partial Y_{g, i}}{\partial x}=h_{D_{i}} S Y_{g, i}
\end{gathered}
$$

In light of the limiting cases discussed above, the rate at which pollutants are converted in the diesel oxidation catalyst can therefore be modeled as the minimum between the rate of mass transport of reactants from the gas phase to the substrate wall and the rate of chemical reaction taking place at surface of the substrate [69, 63]. 


\subsubsection{Energy Balance}

The energy balance for the channel of a diesel oxidation catalyst is obtained considering the convective heat transfer between exhaust gas and substrate wall and the heat generated by the exothermic chemical reactions. The conservation of energy for the exhaust gas flowing in the channel is modeled by the following equation:

$$
O F A \rho_{g} c_{p, g} \frac{\partial T_{g}}{\partial t}+\rho_{g} c_{p, g} u \frac{\partial T_{g}}{\partial x}=h S\left(T_{s}-T_{g}\right)
$$

where $\rho_{g}$ and $c_{p, g}$ denote the density and the specific heat of the exhaust gas respectively, and $h$ represents the convection heat transfer coefficient. Since quasi-steady state operation was assumed, the transient term can be dropped to obtain the following simplified form:

$$
\frac{\partial T_{g}}{\partial x}=\frac{h S}{\rho_{g} u c_{p, g}}\left(T_{s}-T_{g}\right)
$$

The energy balance for the substrate wall is given by equation 2.29:

$$
(1-O F A) \rho_{s} c_{p, s} \frac{\partial T_{s}}{\partial t}=(1-O F A) \lambda_{s} \frac{\partial^{2} T_{s}}{\partial x^{2}}+h S\left(T_{g}-T_{s}\right)+S \sum_{i=1}^{i=4}\left(-\Delta H_{i}\right) r_{i}
$$

In contrast to the gas phase, the time constant for heat transfer in the substrate material is significant and therefore both the transient and conductive terms are included into the energy balance. In equation $2.29 \rho_{s}$ and $c_{p, s}$ denote the density and specific heat of the substrate respectively, $-\Delta H_{i}$ represent the enthalpy of combustion for the $i-t h$ species, and $\lambda_{s}$ is the thermal conductivity of the substrate wall. Values for the enthalpy of combustion are adopted from the work of Sampara [86] and reported in Table 2.2.4.

Boundary conditions for equations 2.28 and 2.29 are as follows:

$$
\begin{gathered}
T_{g}(x=0, t)=T_{g}^{\text {inlet }} \\
\frac{\partial T_{s}}{\partial x}(x=0, t)=\frac{\partial T_{s}}{\partial x}(x=L, t)=0
\end{gathered}
$$




\begin{tabular}{lc}
\hline \hline Reaction & $\Delta H_{i}[\mathrm{~J} / \mathrm{mol}]$ \\
\hline DieselFuel & $-856010^{3}$ \\
$\mathrm{CO}$ & $-28310^{3}$ \\
$\mathrm{NO}$ & $-57.110^{3}$ \\
$\mathrm{C}_{3} H_{6}$ & $-192610^{3}$ \\
\hline \hline
\end{tabular}

while the initial condition for equation 2.29 can be expressed as:

$$
T_{s}(x, t=0)=T_{s, 0}(x)
$$

\subsubsection{Heat and Mass Transfer Coefficients}

The governing equations presented in the previous section assume both heat and mass transfer coefficients to be a known quantity. A work by Shamim [128] showed that there are contradicting reports on the values of Nusselt $(\mathrm{Nu})$ and Sharwood (Sh) numbers to be used in the correlations for heat and mass transfer coefficients in a monolith catalyst: differences in monolith geometry, engine operating conditions and modeling approach all have major influence on the final expressions used in the literature.

The approach followed in this work was to consider general correlations for both Nusselt and Sherwood numbers and to include the resulting constants into the calibration process of the DOC model. The expressions used in this work are as follows:

$$
\begin{aligned}
& N u=A_{N}\left(\operatorname{Re} \operatorname{Pr} \frac{d}{L}\right)^{B_{N}} \\
& S h=A_{S}\left(\operatorname{Re} S c \frac{d}{L}\right)^{B_{S}}
\end{aligned}
$$

where constants $A_{N}, B_{N}, A_{S}$, and $B_{S}$ are determined during the calibration process based on experimental data, as discussed later in this work. 


\subsection{Model Implementation: Elemental Cell Approach}

The governing equations discussed in the previuos sections were implemented following an approach based on modular programming; this technique emphasizes the separation of functionalities into independent, confined modules such that each contains the necessary relationships to solve for one portion of the model. The modules are then connected through interfaces which represent the variables provided to and required by each module. This makes a modular designed system much more reusable than a traditional design.

Based on this programming phylosophy, Matlab-Simulink was selected as the environment to build the model for the diesel oxidation catalyst; each aspect of the DOC was implemented in dedicated blocks (heat transfer, species conservation, etc). Interconnections between each individual block allowed then for simulation of the whole system.

In order to correctly implement the governing equations into the simulation environment, the diesel oxidation catalyst was divided into elemental cells of length $\Delta x$ where properties are assuemed to hold constant during the time step $\Delta t$. The generic elemental cell is depicted in Figure 2.1 while Figure 2.2 represents how connections between multiple cells are established. The number of elemental cells used during the calibration process was arbitrary set to be 10 in order to limit the computational effort needed to solve the set of equations.

Each elemental cell contains modules for the solution of the governing equations; the high level decomposition is translated into two main blocks targeting the species conservation and energy balance respectively. The structure of each elemental cell is depicted in Figure 2.3. The two main blocks are interconnected as species conservation and energy balance are coupled sets of equations and need to be solved simultaneously. 


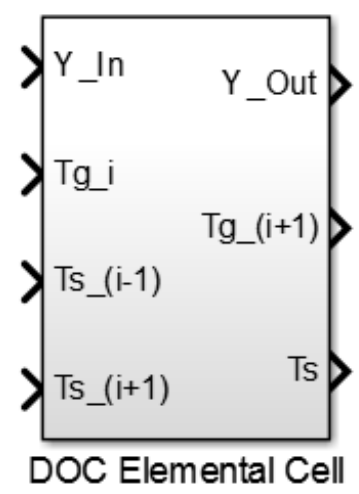

Figure 2.1: DOC Elemental Cell.

\subsubsection{Energy Equations}

It is worth noting that the elemental cell at the generic position $i$ requires temperature signales from both cells at $i-1$ and $i+1$; this fact is a consequence of the scheme that was used to implement the energy equation of the solid phase. The adopted approach was aimed at implementing a second order accurate scheme by replacing the derivatives by a central difference approximation. A possible method to simulate the thermal inertia of the monolith would be to divide the structure into $n$ elemental cells, each one characterized by its own heat capacity, and then solve for $n$ energy balances . This approach does not require modeling of heat conduction as the time delay in temperature variation for front

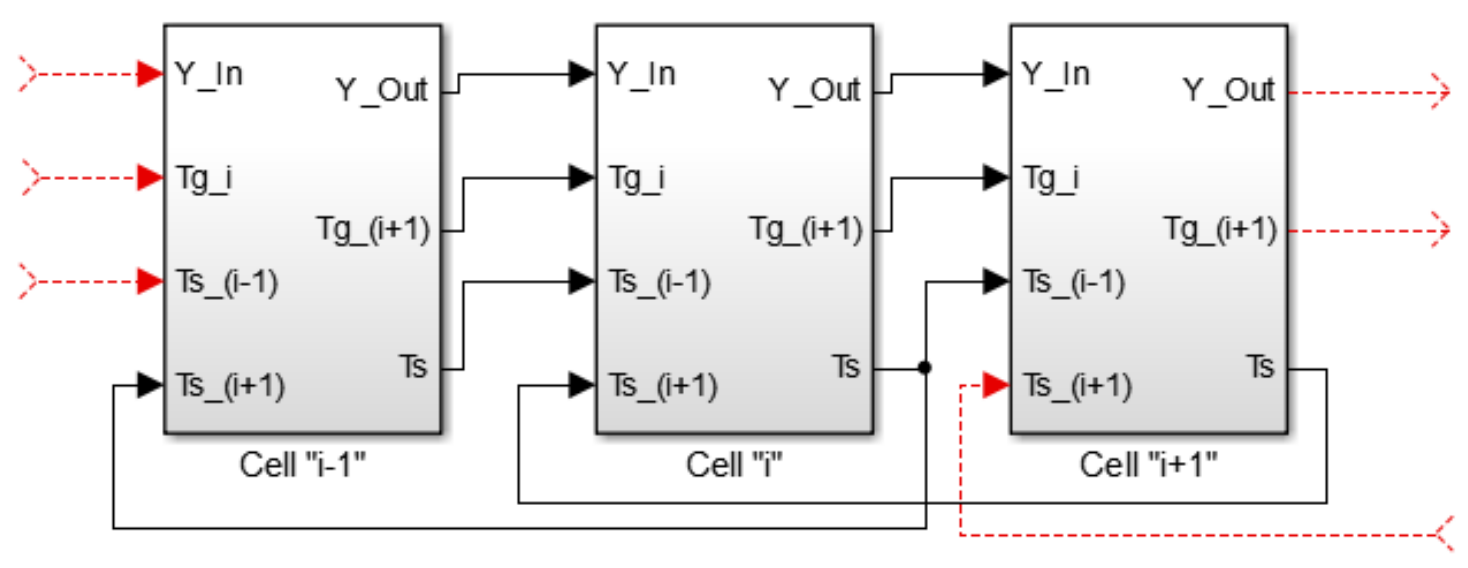

Figure 2.2: Connections between DOC Elemental Cells. 


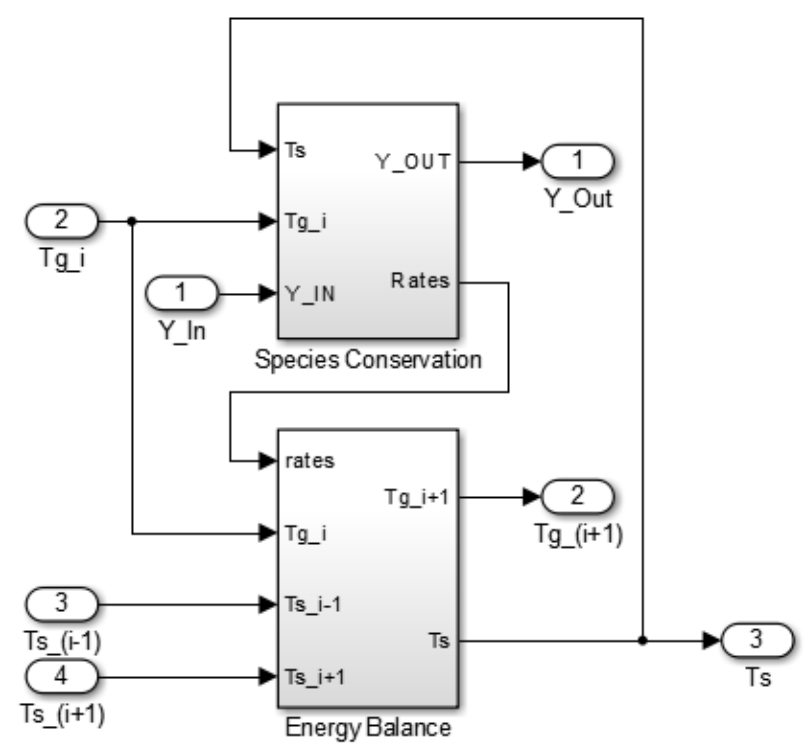

Figure 2.3: Modules for Species Conservation and Energy Balance.

and back of the monolith is simulated via insertion of the $n$ heat capacities. However, heat conduction in the solid phase plays a major role in case of DOCs and DPFs due to the low conductivity of the ceramic substrate. The terms $i-1$ and $i+1$ arise from terms representing heat conduction in and out of the element.

The module computing the amount of heat flowing into the elemental cell through conduction is based on the following approximated expression:

$$
\dot{Q}_{\text {cond }}^{i n}=-\frac{\lambda_{s}(1-O F A)}{\Delta x^{2}}\left(T_{s, i}-T_{s, i-1}\right)
$$

Similarly, heat flowing out from element $i$ to element $i+1$ is given by:

$$
\dot{Q}_{\text {cond }}^{\text {out }}=-\frac{\lambda_{s}(1-O F A)}{\Delta x^{2}}\left(T_{s, i+1}-T_{s, i}\right)
$$

The reliability of the approach discussed above was tested against a more traditional method based on the Crank-Nicolson scheme. A full Federal Testing Procedure (FTP) engine transient cycle was simulated using both thermal modules and results were compared to check if the approximation used to model heat conduction was in agreement 
with a second order accurate scheme. Comparison of results obtained with these two methodologies is given in Figure 2.4, Figure 2.5, and Figure 2.6 which show simulated substrate temperature at different locations over the length of the DOC.

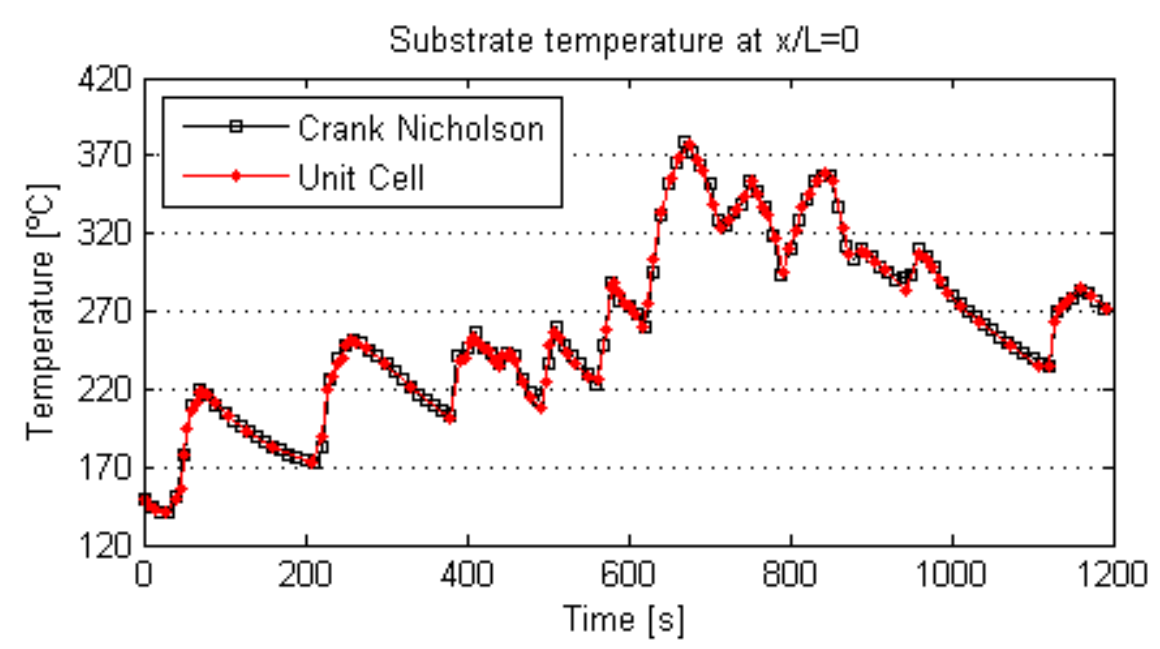

Figure 2.4: Simulated substrate wall temperature during an FTP cycle: case $x / L=0$.

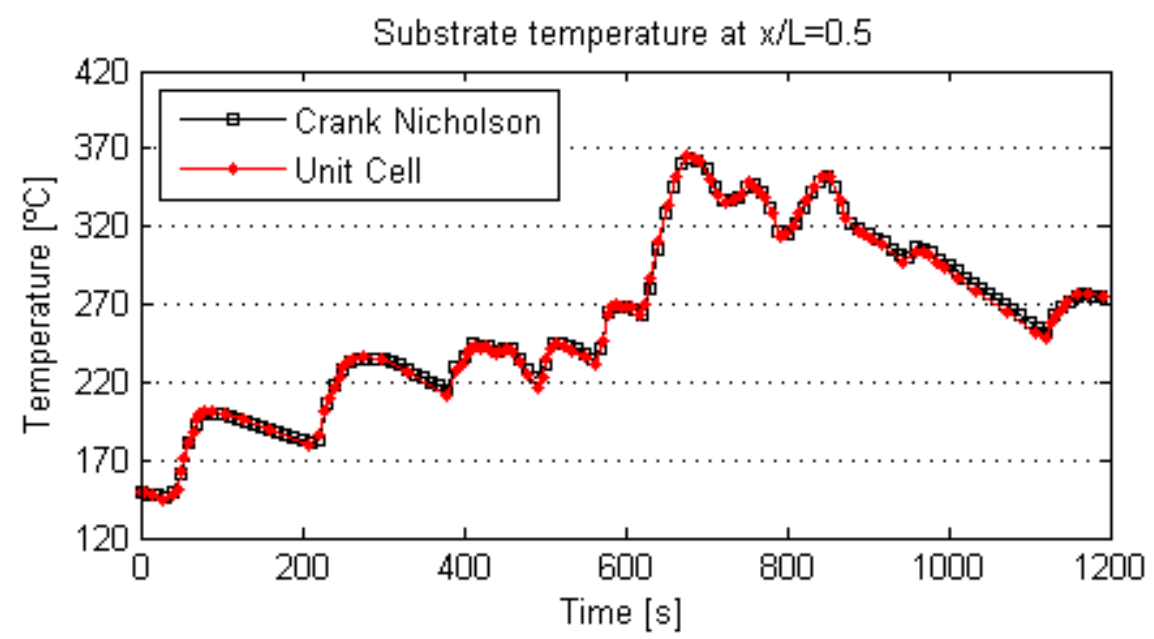

Figure 2.5: Simulated substrate wall temperature during an FTP cycle: case $x / L=0.5$.

At the first time step $t$ the temperature of the substrate wall is known via initial condition expressed by equation 2.32; therefore, energy equation for the gas phase (equation 2.28) can be solved for $T_{g}$ using a methodolgy presented in [129] and based on local analytical solution of equation 2.28 via NTU method. The implemented solution is therefore as follows:

$$
T_{g, i+1}=T_{s, i}+\left(T_{g, i}-T_{s, i}\right) e^{-N T U}
$$




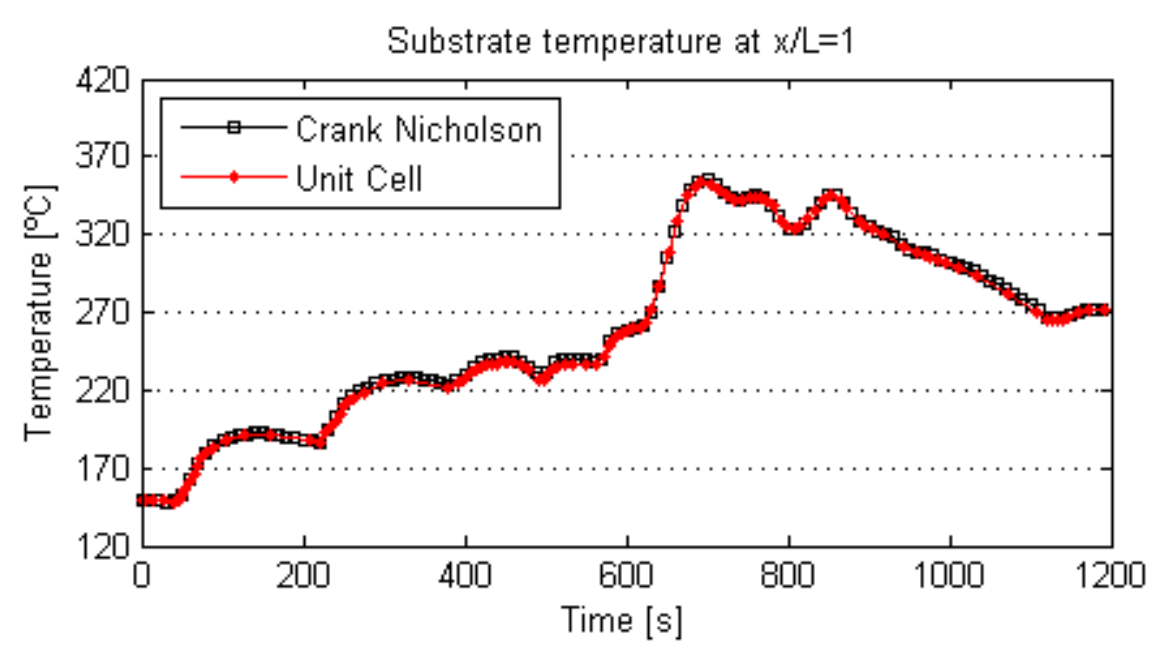

Figure 2.6: Simulated substrate wall temperature during an FTP cycle: case $x / L=1$.

with

$$
N T U=\frac{h S}{\dot{m}_{g} c_{p, g}}
$$

Once the temperature of the gas phase is computed, the substrate temperature can be updated using the approach discussed earlier in this section.

\subsubsection{Species Conservation}

As long as species conservation is concerned, the implementation of the governing equations followed two different approaches for the complete and simple models. In case of the complete model the solution for the species conservation involves both surface and bulk concentrations; at any time step $t$, with known values for $T_{g, i}$ and boundary condition for $Y_{g, i}$, equation 2.21 contains only unknowns $Y_{s, i}$. This is a system of non-linear algebraic equations that can be solved using the Newton's method [129].

Once the concentration of the reacting species at the substrate wall is computed, integration of equation 2.20 over the monolith segment $\Delta x$ leads to the following expression [129]:

$$
Y_{g, i+1}=Y_{s, i}+\left(Y_{g, i}-Y_{s, i}\right) e^{\frac{-h_{D} \rho S}{\dot{m}_{g}}}
$$

On the other hand when the simple model is employed, the approach based on limiting cases is used to simplify the governing expressions and the resulting equations 2.24 
and 2.26 can be readily integrated over the monolith segment $\Delta x$. 


\section{Chapter 3}

\section{Modeling of Diesel Particulate Filter}

\subsection{General Structure}

The model hereby is an evolution of a previous work presented by Mulone et al. [63] which incorporates the findings of Premchand et al. [130], Bisset [108] and Konstandopoulos et al.[57]. The model is an in-house 1-D representation of a diesel particulate filter over the wall thickness and it is the result of the collaboration between West Virginia University and the University of Rome "Tor Vergata". According to the 1-D approach, exhaust flow and filter properties are assumed to be constants over the DPF length. Although this is not the case in real filters, for the sake of the development of a filtration and regeneration model the 1-D approach is usually regarded as a good compromise between the obtained degree of detail and the required computational effort. Further, as suggested by Opris in [58], the soot loading procedure is such that cake layer formation is auto-adjusted from the standpoint of soot longitudinal distribution; in fact, if there are zones of the channels which are more loaded with respect to others, these zones will also generate a greater pressure drop. The main consequence of this fact is that the flow within the channels will "correct" itself in order to follow the path of least resistance, thus focusing the soot loading on portions of the channels that were initially characterized by a lower amount of PM mass. Consequently, the particulate layer thickness will change in a quasi-isotropic manner making reasonable the choice of a less spatially developed 
approach.

The numerical code of the filtration and regeneration models was built using Matlab language and then included in the Simulink environment. The code consists of a main program which uses several dedicated subroutines to sequentially solve for filter properties and soot loading (Figure 3.1):

- Input File and Constants Definition: the code loads two external files containing information about the engine cycle to be simulated and values for the main constants used in the code itself.

- Compute Flow Properties: main flow properties based on exhaust composition and temperature are calculated and stored by this subroutine.

- Filtration Model: the code solves for the filtration process taking place in both filter walls and cake layers, storing a temporary value for the PM mass trapped in the filter.

- Regeneration Model: the code applies the regeneration theory based upon both thermal and catalytic soot oxidation by $\mathrm{NO}_{2}$ and $\mathrm{O}_{2}$, computing the final amount of PM mass trapped in the filter used to upgrade filter properties.

- Energy Model: this subroutine computes the energy balance of the whole filter, taking into account also the heat generated by the oxidation of soot, to upgrade filter wall temperature. 


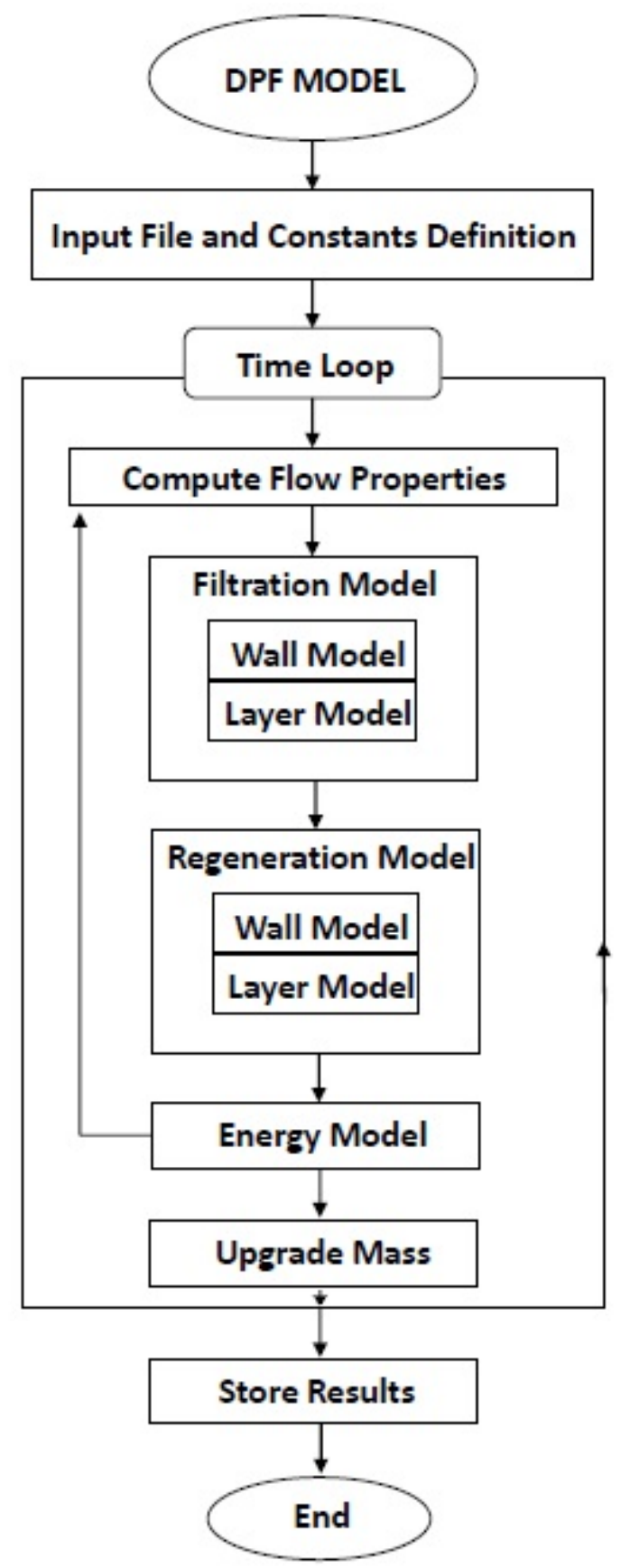

Figure 3.1: Code Structure. 


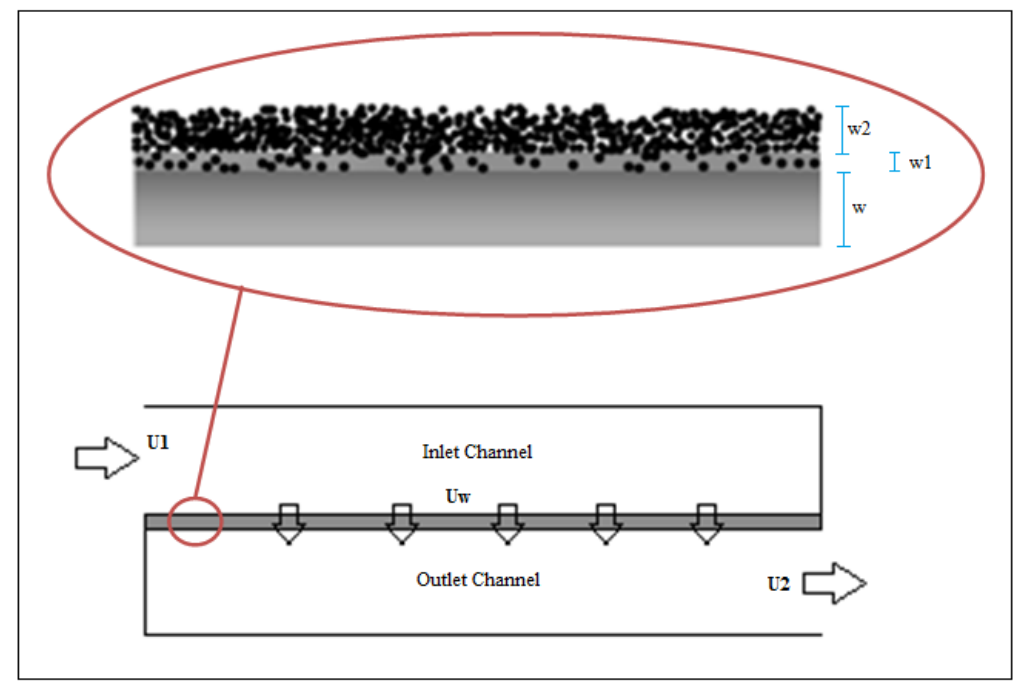

Figure 3.2: Representation of the Computational Volume.

The unit-cell analyzed by the filtration and regeneration model is a single pair of inletoutlet channels (Figure 3.2) assumed to be representative of the whole filter under the assumption of uniform inlet conditions [130, 57, 108].

The main assumption used in the development of the DPF model are listed below:

- The system can be modeled using a quasi steady-state approach. This assumption is valid considering the relatively short residence time required for the exhaust gas to pass through the particulate filter.

- Concentrations of gaseous species, exhaust temperature and velocity are referred to the cross section of the channel (Figure 3.2) and are considered to be constant over the filter length.

- The temperature of the exhaust gas leaving the DPF is assumed to be equal to the temperature of the substrate wall. This assumption is made possible considering the relatively high thermal conductivity of the substrate wall and soot particles [57].

- PM mass trapped in the filter wall and in the washcoat/cake layers is assumed to have the same temperature of the substrate wall [131]. 


\subsection{Wall Sub-model}

The wall sub-model comprises transient soot particle filtration as well as wall regeneration due to thermal oxidation of soot by $\mathrm{O}_{2}$ and $\mathrm{NO}_{2}$ according to the framework discussed in [63, 132] and based on [122, 133, 107]. However, differently from previous studies, this work presents a correlation that links the thickness of the cake/washcoat layer to the wall filtration efficiency.

\subsubsection{Continuous Filtration Theory}

The filtration theory developed in this study is an extension of the "unit collector theory" $[111,57]$ which has been modified to be analytically represented [63, 132]. The analytical treatment is a feature of the presented approach that allows for detailed representation of the wall transient behavior.

According to the theory presented by Konstandopoulos et al. [111], the microstructure of the substrate wall can be represented as formed by packed individual spherical cells of characteristic dimension $b$ (Figure 3.3), each one hosting a spherical "unit collector" with initial size (clean filter) $d_{c, 0}$ assumed to collect PM particles by the mechanisms of Brownian diffusion and direct interception as the exhaust gas flows through the filter wall.

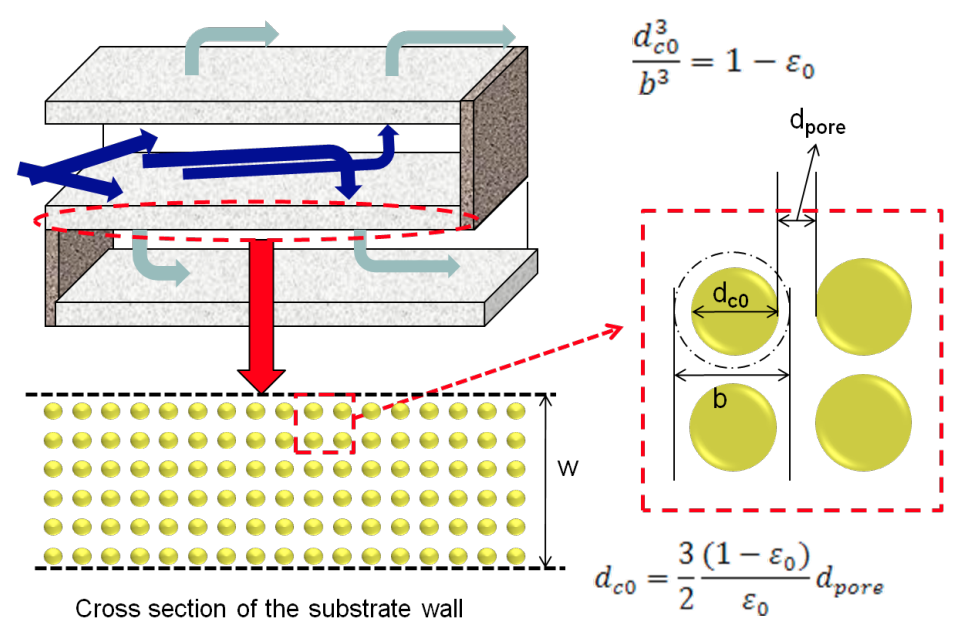

Figure 3.3: Representation of the unit collector theory from [134]. 
The initial collector size can be geometrically related to the unit cell dimension by the wall porosity of the clean filter according to equation 3.1, while the correlation with the substrate pore size is given by equation 3.2 :

$$
\begin{gathered}
b^{3}=\frac{d_{c, 0}{ }^{3}}{1-\varepsilon_{0}} \\
d_{c, 0}=\frac{3}{2}\left(\frac{1-\varepsilon_{0}}{\varepsilon_{0}}\right) d_{\text {pore }}
\end{gathered}
$$

The Brownian collection efficiency of a single unit collector is given by a relationship between the Peclet number (equation 3.4) and a geometrical function known as "Kuwabara unit cell function" [57], reported in equation 3.5:

$$
\begin{gathered}
\eta_{D}=3.5 g(\varepsilon) P e^{-\frac{2}{3}} \\
P e=\frac{U_{i} d_{c}}{D_{p}} \\
g(\varepsilon)=\left[\frac{\varepsilon}{2-\varepsilon-\frac{9}{5}(1-\varepsilon)^{\frac{1}{3}}-\frac{1}{5}(1-\varepsilon)^{2}}\right]^{\frac{1}{3}}
\end{gathered}
$$

where $U_{i}$ denotes the interstitial velocity which can be expressed as a function of the wall porosity and wall appraoch velocity $u_{w}$ as:

$$
U_{i}=\frac{u_{w}}{\varepsilon}
$$

The particle diffusivity appearing in equation 3.4 can be computed as: 


$$
D_{p}=S C F \frac{k_{b} T}{3 \pi \mu d_{p}}
$$

where $k_{b}$ is the Boltzmann's constant, $T$ is the absolute temperature of the exhaust, $\mu$ is the dynamic viscosity of the exhaust gas, $d_{p}$ is the PM particle size and the $S C F$ is the Stokes-Cunningham slip correction factor which takes into account slip flow conditions when the gas mean free path has dimensions which are comparable with the mean pore size of the filter wall. A relationship for the $S C F$ is given below:

$$
S C F=1+K n_{p}\left(1.257+0.4 e^{-\frac{1.1}{K n_{p}}}\right)
$$

The Knudsen number appearing in equation 3.8 is computed as follows:

$$
K n_{p}=\frac{2 \lambda}{d_{p} \text { ore }}
$$

and the mean free path $(\lambda)$ is defined as:

$$
\lambda=\frac{\mu}{P} \sqrt{\frac{\pi R T}{2 M W}}
$$

In the previuous equation, $M W$ denotes the molecular weight of the exhaust gas, $R$ indicates the universal gas constant, $P$ the absolute pressure and $\mu$ the kinematic viscosity of the gas.

The second collection mechanism assumed to take place in the substrate wall is the "direct interception" [111]. The related collection efficiency can be expressed as a function of the "Kuwabara function" and an interception parameter $\left(N_{R}\right)$ defined as the ratio 
between pore and collector sizes:

$$
\eta_{R}=1.5 N_{R}^{2} \frac{[g(\varepsilon)]^{3}}{\left(1+N_{R}\right)^{\frac{3-2 \varepsilon}{\varepsilon}}}
$$

Assuming the two collection mechanisms to be independent of each other, the global collection efficiency can be computed as:

$$
\begin{gathered}
\eta_{D R}=\eta_{D}+\eta_{R}-\eta_{D} \eta_{R} \\
N_{R}=\frac{d_{p}}{d_{c}}
\end{gathered}
$$

The equations discussed so far can be employed to model the filtration of PM particles on a number basis. In fact, both diffusion and interception collection mechanisms are a function of the particle size $\left(d_{p}\right)$ through the diffusion coefficient (3.7) and the interception parameter (3.13). Further, filtration mechanism depends upon properties of the substrate, such as the wall porosity, which can vary during the loading cycle leading to a distribution of the porosity over the wall thickness. Under quasi steady-state assumption, the number concentration of the $i$-th particle size decreases over the wall thickness following the relationship:

$$
\frac{d N_{i}(x, t)}{d x}=-f_{N}(x, t) N_{i}(x, t)
$$

where $N_{i}(x, t)$ denotes the particle number concentration of the $i$-th size at time $t$ for a general position $x$ within the wall thickness, and $f_{N}(x, t)$ is a function of the substrate properties and the combined collection efficiency $\eta_{D R}$ : 


$$
f_{N}(x, t)=\frac{3}{2} \frac{\eta_{D R}(x, t)[(1-\varepsilon(x, t))]}{d_{c}(x, t) \varepsilon(x)}
$$

In the most general case, properties reported in equation 3.15 are a function of both time and wall thickness. The deposition profile in terms of particle number can be computed by integration of equation 3.14 taking into account that $f_{N}(x, t)$ is a function of $x$ and can be represented, from a computational standpoint, as a polynomial of order $n:$

$$
N_{i}(x, t)=N_{i}^{x=0} \mathrm{e}^{-\int_{0}^{x} f(x, n) d x}
$$

where the number count for the $i_{t} h$ particle size at the filter inlet serves as boundary condition. In the particular case of clean filter, however, wall properties can be assumed to be homogeneous over the wall thickness and therefore the same equation can be easily integrated to give the deposition profile for the first time step:

$$
N_{i}(x)=N_{i}^{x=0} \mathrm{e}^{-f x}
$$

The condition of "clean" substrate is lost as soon as PM particles start to flow through the filter and are trapped due the collection mechanisms introduced earlier. As PM mass is accumulated in the filter, wall pore size decreases leading to a reduced overall porosity. This leads to an enhancement of the collection efficiency as particles can flow in a restricted path. The upgraded wall properties can be computed using the same equations cited above once the collected PM mass has been calculated. The evolution of the wall collection efficiency as a function of the particle size during a typical loading cycle can be observed in Figure 3.4.

In order to take into account the different contribution of each particle size to the trapped mass distribution, collection efficiency on a mass basis has been defined by integration of PM size distribution across the entire particle spectrum. This method, differ- 


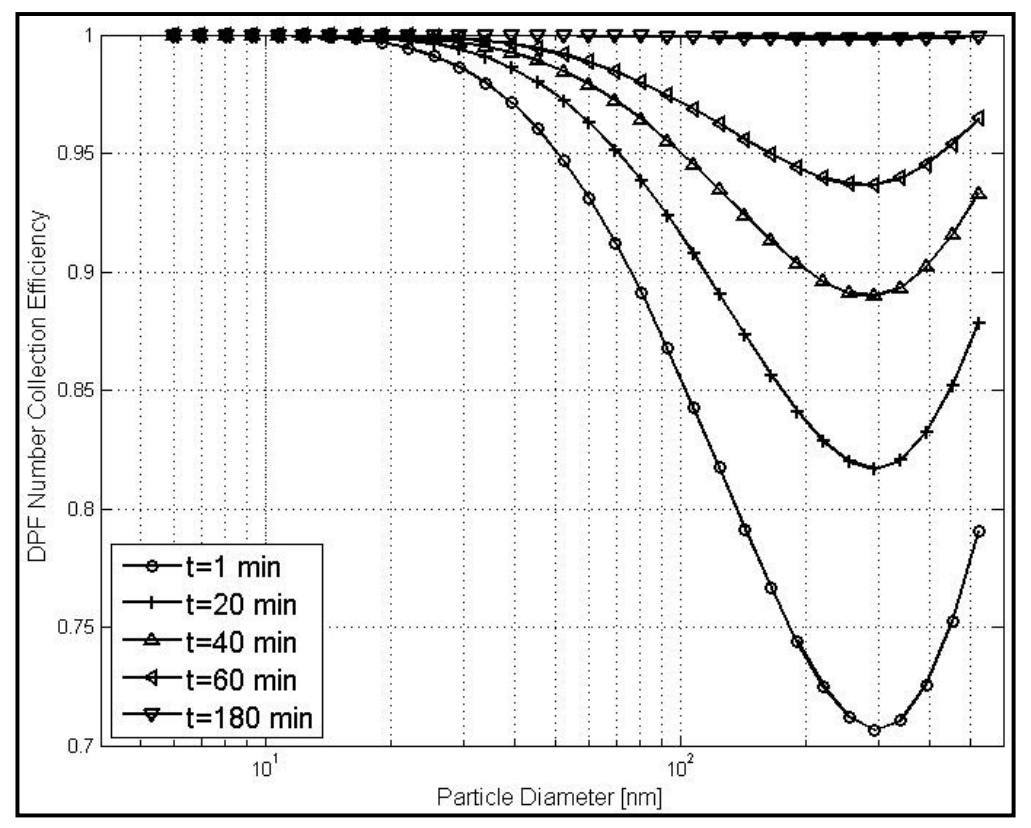

Figure 3.4: Evolution of particle collection efficiency during a loading process.

ently from what presented by Mohammed et al. [135] for example, offers the possibility to treat directly PM mass concentrations expressed as $P M_{\text {mass }} /$ Gas volume $_{\text {en }}$ thus allowing the wall submodel to use data collected by a PM soot sensor without going through the calculation of any particle density which, in general, can be a function of engine operation and fuel composition.

The PM mass collection efficiency can therefore be computed as:

$$
\eta_{\text {mass }}(x)=\frac{\sum_{i=1}^{i=n} \eta_{D R}^{i}(x) N_{i}(x) d \log D_{p} d_{i}^{3}}{\sum_{i=1}^{i=n} N_{i}(x) d \log D_{p} d_{i}^{3}}
$$

where the upper limit $n$ of the summation represents the last bin of the particle spectrometer used to collect the particle size distribution. The introduction of an overall mass collection efficiency enables for the definition of a governing equation for the mass filtration process. Following an approach similar to the one presented for the case of particle number, PM mass concentration decreases over the wall thickness according to:

$$
\frac{d C_{m}(x)}{d x}=-f_{m a s s} C_{m}(x)
$$


where $C_{m}$ denotes the PM mass concentration and $f_{\text {mass }}$ is a combination of the mass collection efficiency and wall properties:

$$
f_{\text {mass }}(x)=\frac{3}{2} \frac{\eta_{\text {mass }}(x)[(1-\varepsilon(x))]}{d_{c}(x) \varepsilon(x)}
$$

Concentration profiles over the wall thickness can be computed by integration of equation 3.19 where $f_{\text {mass }}$ is a function of the wall thickness $x$ and can be represented by a polynomial of $n-t h$ order.

Denoting with $Q_{c h}$ the exhaust volumetric flow rate related to one channel of the filter, the differential equation for the rate of mass collection is given by:

$$
\frac{d \dot{m}_{p}}{d x}=\frac{d C_{m}}{d x} Q_{c h}=f_{m a s s}(x) C(x) Q_{c h}
$$

whose integration over the $x$-domain leads to the total amount of PM mass collected by wall filtration in one channel:

$$
m_{\text {wall }}=\int_{0}^{w} f(x) C_{m}(x) Q_{c h} d x
$$

In order to upgrade the filter wall properties to take into account the reduction in porosity, an expression to correlate the mass trapped by the wall to the mass trapped by a single collector is needed:

$$
n_{\text {cell }}=\frac{a L w\left(1-\varepsilon_{0}\right)}{\frac{4}{3} \pi\left(\frac{b}{2}\right)^{3}}=\frac{a L w\left(1-\varepsilon_{0}\right)}{V_{\text {cell }}}
$$

where $a$ denotes the channel width, $L$ represents the channel length, $w$ is the wall thickness and $b$ the size of a unit cell. Assuming the microstructure of the substrate to be continuous over the $x$-direction, the rate of PM mass collection over a single collector can be expressed as: 


$$
\frac{d \dot{m}_{p}}{d n}=\frac{d \dot{m}_{p}}{d x} \frac{d x}{d n}=f_{\text {mass }}(x) C(x) Q_{c h} \frac{V_{\text {cell }}}{a L\left(1-\varepsilon_{0}\right)}
$$

Once the deposition rate for a single collector has been computed, it is possible to calculate the increase in collector size as:

$$
d_{c}(x, t)=2\left(\frac{3}{4 \pi} \frac{m_{\text {coll }}(x, t)}{\rho_{\text {soot }, w}}+\left(\frac{d_{c 0}}{2}\right)^{3}\right)^{\frac{1}{3}}
$$

where $\rho_{\text {soot }, w}$ is the apparent soot packing density in the wall thickness. The increase in collector size affects the wall porosity and permeability. The upgraded values for these two properties can be computed as:

$$
\begin{gathered}
\varepsilon(x, t)=1-\left(\frac{d_{c}(x, t)}{d_{c 0}}\right)^{3}\left(1-\varepsilon_{0}\right) \\
\frac{k(x, t)}{k_{0}}=\left(\frac{d_{c}(x, t)}{d_{c 0}}\right)^{2} \frac{f(\varepsilon)}{f\left(\varepsilon_{0}\right)}
\end{gathered}
$$

where the "Kuwabara" hydrodynamic factor is a function of wall porosity and is expressed as $[57]$ :

$$
f(\varepsilon)=\frac{9}{2} \frac{\left(2-\frac{9}{5}(1-\varepsilon)^{\frac{1}{3}}-\varepsilon-\frac{1}{5}(1-\varepsilon)^{2}\right)}{1-\varepsilon}
$$

\subsubsection{Partition between wall and cake layer PM mass}

Depending upon the particular filter condition, PM particles entering the DPF may be collected either in the filter wall by the "deep bed filtration" mechanism or on the wall surface forming a thin layer of soot which becomes thicker as more PM mass is deposited. In case of "deep bed" filtration, particles enter the substrate wall and are collected according to the mechanisms presented in the previous section. However, as the wall becomes more and more loaded some PM particles may start to deposit directly 


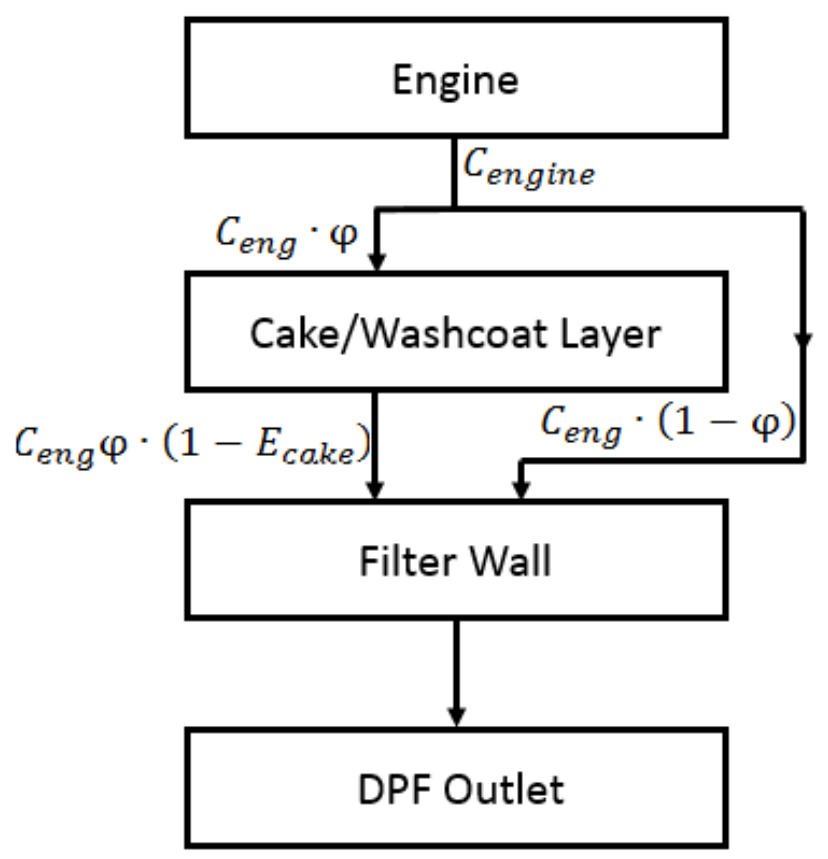

Figure 3.5: PM mass balance: partition in wall and cake layer.

on the wall surface. This thin layer of deposited mass eventually starts to act as an additional filter membrane preventing PM particles to reach the substrate wall. This thin layer of soot can be characterized by a dedicated filtration efficiency as suggested in $[135]$ :

$$
E_{\text {cake }}(t)=A_{\eta}\left(1-\mathrm{e}^{-\eta_{c} \frac{w_{c a k e}}{d_{c \text { cake }}}}\right)
$$

where $A_{\eta}$ is a tuning parameter used to prevent cake filtration efficiency to reach the unity in case of large cake later thicknesses, $\eta_{c}$ is the collection efficiency for a collector in the cake layer. The size of the unit collectors in the cake layer can be assumed to be equal to the average size of the upstream particles in first approximation.

The mass balance needed to estimate the amount of PM mass entering the filter wall and the cake layer is represented in Figure 3.5.

$$
C_{\text {wall }}(t)=C_{\text {eng-out }}\left[1-\phi(t) E_{\text {cake }}(t)\right]
$$




$$
C_{\text {cake }}(t)=C_{\text {eng-out }}\left[\phi(t) E_{\text {cake }}(t)\right]
$$

The PM mass concentration entering the filter is at first divided in two components by using the "partition coefficient" defined in [106] and computed as equation 3.32. This coefficient ranges between 0 (clean filter, pure deep bed filtration) and 1 (pure cake layer deposition) and its value is determined considering the wall surface condition $(x=0)$ which is representative of the collection mechanism. The parameter $\psi$ is a dimensionless constant used to determine the maximum possible size for a loaded collector as fraction of the unit spherical cell $(b)$ used to represent the wall microstructure.

$$
\phi(t)=\left(\frac{\left(d_{c}(0, t)\right)^{2}-d_{c 0}^{2}}{(\psi b)^{2}-d_{c 0}^{2}}\right)
$$

The portion of entering PM mass that is used for the cake layer is then processed using the cake filtration efficiency to determine the extent of cake deposition, taking into account the cake layer thickness itself.

The equation presented above correlate the superficial effect modeled by the partition coefficient with the cake filtration efficiency in a smooth manner thus allowing a continuous switch between deep bed filtration to pure cake deposition. Moreover, the PM mass entering the substrate wall becomes independent of the wall characteristics as it is governed only by the cake filtration efficiency after long loading time. This characteristic allows for ash modeling.

Previous studies ([56]) have shown how the deep bed filtration mechanism is inhibited in aged filters as consequence of cyclic loading/regeneration events. The inhibition mechanism is the result of a thin membrane of unburnt soot or ash that builds up on the wall surface and is not removed during the regeneration process, even if the substrate wall undergoes a full regeneration. As result, new PM particles are prevented to reach the surface of the wall. The adoption of the sole "partition coefficient" may not be enough in order to replicate this behavior as the amount of mass entering the wall is ultimately a function of the wall collector size which changes according to the amount of PM mass 
contained in the wall itself. After a successful regeneration event the partition coefficient could become lower than 1 thus allowing new PM mass to reach the wall. On the other side, the introduction of a link between partition coefficient and cake filtration efficiency allows to model filter aging after successive loading/regeneration cycles.

\subsubsection{Continuous Wall Regeneration}

The regeneration process in the substrate wall is assumed to deplete particulate matter by thermal means due to $\mathrm{O}_{2}$ and $\mathrm{NO}_{2}$ oxidation. The oxidation of PM soot by catalytic means is assumed to be confined to the washcoat layer which, as presented in [57, 121], is modeled as a soot layer building up on top of the wall surface.

Since PM mass is collected in the wall with a continuous change in deposition density, the soot oxidation model developed by Bisset [108, 131] and Konstandopoulos [136] needed to be to improved in order to be consistent with the continuous analytical treatment characterizing the filtration theory. In fact, differently from past studies, the soot oxidation theory cannot be applied in a discrete manner since the wall thickness is not divided into slabs where properties are assumed to be constant but, on the contrary, they change continuously.

To that aim, the PM mass trapped in the filter wall is represented as an equivalent soot layer computed per unit wall length. This quantity, denoted as $d w / d x$ and already presented by the same author in [132] and [63], is very important in order to treat regeneration in cake layer and in the wall in a unified fashion, allowing to use same definitions for the reaction rates.

$$
\frac{d w}{d x}=\left(\frac{1}{\rho_{\text {soot }, w}}\right)\left(\frac{1-\varepsilon_{0}}{V_{c e l}}\right) \frac{d m_{p}}{d x}
$$

In particular, $d w / d x$ in the washcoat and cake layers is always equal to unity as it is the derivative of a parameter with respect to itself, while it is a function of both time and wall thickness in the substrate wall. A graphic representation of this parameter is 


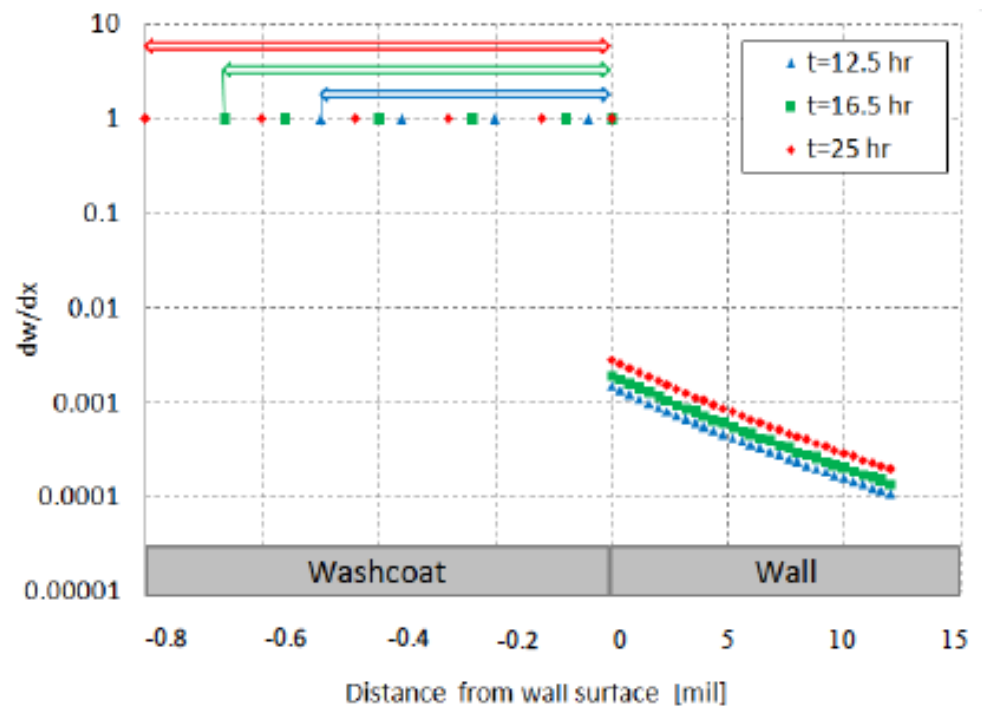

Figure 3.6: Graphic representation of $d w / d x$ in both washcoat layer and substrate wall. given in Figure 3.6 from [63].

The different behavior of this parameter can be summarized considering that the wall can collect soot in a constant thickness by varying the value of $d w / d x$, while on the contrary the cake/washcoat layer collects soot by varying thickness and keeping constant $d w / d x$. Since depletion rates are proportional to the soot layer thickness, mass conservation for the gas species in the wall may be written as:

$$
\begin{gathered}
\frac{d}{d x}\left(\rho_{w} v_{w} Y_{O_{2}, w}\right)=-S_{p} \rho_{w} Y_{O_{2}, w} k_{t h, O_{2}}\left(1-\frac{f_{C O}}{2}\right) \frac{d w}{d x}=\frac{d R_{O_{2}, w}}{d x} \\
\frac{d}{d x}\left(\rho_{w} v_{w} Y_{N O_{2}, w}\right)=-S_{p} \rho_{w} Y_{N O_{2}, w} k_{t h, N O_{2}}\left(2-g_{C O}\right) \frac{d w}{d x}=\frac{d R_{N O_{2}, w}}{d x}
\end{gathered}
$$

where all the variables are presented and discussed in the section "Layer Sub-model" where the whole regeneration theory is presented in detail.

Molar fractions of $\mathrm{O}_{2}$ and $\mathrm{NO}_{2}$ can be obtained by integration of equations 3.34 and 3.35 across the wall thickness: 


$$
\begin{gathered}
Y_{O 2, w}(x)=Y_{O 2, \text { Inlet }} \exp \left(\frac{-S_{P} k_{t h, O 2}\left(1-\frac{f_{C O}}{2}\right) \int_{0}^{x}\left(\frac{d w}{d x}\right)}{v_{w}}\right) \\
Y_{N O 2, w}(x)=Y_{N O 2, \text { Inlet }} \exp \left(\frac{-S_{P} k_{t h, O 2}\left(1-g_{C O}\right) \int_{0}^{x}\left(\frac{d w}{d x}\right)}{v_{w}}\right)
\end{gathered}
$$

The time rate of change of a soot layer with fixed density during soot depletion via $\mathrm{O}_{2}$ and $\mathrm{NO}_{2}$ oxidation can be written as:

$$
\frac{\partial}{\partial t}\left(\rho_{\text {soot-lyr }} w\right)=-\left[\frac{M_{c}}{M_{O_{2}}}\left(\frac{R_{O_{2}, w}}{1-\frac{f_{C O}}{2}}\right)+\frac{M_{c}}{M_{N O_{2}}}\left(\frac{R_{N O_{2}, w}}{2-g_{C O}}\right)\right]
$$

hence, the time rate of change of the distribution profile of the equivalent soot layer in the wall $d w / d x$ can be obtained by derivation of equation 3.38 with respect to the wall thickness $w$ under the assumption of quasi steady-state conditions:

$$
\frac{\partial}{\partial t}\left(\rho_{\text {soot-lyr }} \frac{\partial w(x)}{\partial x}\right)=-\left[\frac{M_{c}}{M_{O_{2}}}\left(\frac{\frac{\partial\left(R_{O_{2}, w}\right)}{\partial x}}{1-\frac{f_{C O}}{2}}\right)+\frac{M_{c}}{M_{N O_{2}}}\left(\frac{\frac{\partial\left(R_{N O_{2}, w}\right)}{\partial x}}{2-g_{C O}}\right)\right]
$$

Finally, once the equivalent soot layer has been computed it is possible to convert this value back to distribution of mass and upgrade the collectors size in the substrate wall as well as filter properties such as permeability and porosity.

\subsection{Layer Sub-Model}

The "Layer Sub-Model" applies soot oxidation theory to the cake and washcoat layer, in a similar fashion to what was presented by Bisset [108] and Konstandopoulos [57]. In fact, as already discussed in the previous section, as exhaust gas flows through the particulate filter, PM particles may eventually be collected directly on top of the wall surface, forming one or two layers depending upon the substrate nature. Referring to Figure 3.2, if the substrate is loaded with catalytic coating the "2-Layers" can be applied 
and the soot deposition is assumed to start in a layer $\left(w_{1}\right)$ where both catalytic and thermal oxidation take place. When this washcoat layer reaches a predetermined maximum thickness, particles are assumed to enter a second layer $\left(w_{2}\right)$ where oxidation due to catalytic means can be assumed to be negligible due to the distance from the active coating. On the other hand, in case the substrate wall is not loaded with any catalytic material the soot layer is assumed to build up directly in a cake later undergoing soot oxidation due to thermal means only.

\subsubsection{PM Thermal Oxidation Kinetics: Cake Layer}

In the cake layer, soot oxidation is assumed to be driven by thermal means only. Diesel exhaust comprises of two gases which are suitable for soot oxidation: $\mathrm{O}_{2}$ and $\mathrm{NO}_{2}$. Although the available $\mathrm{NO}_{2}$ concentration is typically much lower than the available $\mathrm{O}_{2}$ (due the lean combustion of Diesel), oxidative reaction pathways due to $\mathrm{NO}_{2}$ cannot be disregarded considering the significant difference in activation energy when compared to oxygen. In fact, thermal oxidation of soot particles due to $\mathrm{O}_{2}$ has been shown to take place at extremely high exhaust temperatures $\left(500-600^{\circ} \mathrm{C},[57]\right)$ while soot oxidation by $\mathrm{NO}_{2}$ may take place already at temperatures $150^{\circ} \mathrm{C}$.

Although transport processes in a DPF occur between both solid and gas phase, in this study chemical reactions in the gas phase are neglected in the cake layer. Oxidation of $\mathrm{NO}$ back to $\mathrm{NO}_{2}$ is however modeled in the washcoat layer similarly to what presented by Mohammed [135]. Assuming soot particles to be purely carbonaceous, soot oxidation reactions due to $\mathrm{O}_{2}$ can be written as:

$$
\begin{aligned}
& \mathrm{C}+\mathrm{O}_{2} \rightarrow \mathrm{CO}_{2} \\
& \mathrm{C}+\frac{1}{2} \mathrm{O}_{2} \rightarrow \mathrm{CO}
\end{aligned}
$$


or, in terms of a global 1-step heterogeneous reaction:

$$
C+\left(1-\frac{f_{C O}}{2}\right) O_{2} \rightarrow f_{C O} C O+\left(1-f_{C O}\right) C O_{2}
$$

where the carbon monoxide selectivity $f_{C} O$ has the following dependance:

$$
f_{C O}=\frac{1}{1+k_{t h} y_{O_{2}}^{n 1} \exp \left(-\frac{E_{f_{1}}}{R T_{w}}\right)}
$$

The selectivity depends on the oxygen molar fraction that contributes to thermal regeneration $y_{\mathrm{O}_{2}}^{n 1}$, exhaust temperature in the cake layer (assumed to be equal to the wall temperature $T_{w}$ ) and activation energy $E_{f_{1}}$. The reaction rate constant $k_{t h}$ is assumed to be of an Arrhenius form:

$$
k_{t h}=A_{t h} T_{w}^{n} \exp \left(-\frac{E_{t h}}{R T_{w}}\right)
$$

where $A_{t h}$ is the pre-exponential factor for oxidation due to thermal means while the group $\left(-\frac{E_{t h}}{R T_{w}}\right)$ is the activation temperature of the thermal oxidation process.

Similarly, soot oxidation by $\mathrm{NO}_{2}$ can be modeled as follows [131, 62]:

$$
\begin{gathered}
\mathrm{C}+2 \mathrm{NO}_{2} \rightarrow \mathrm{CO}_{2}+2 \mathrm{NO} \\
\mathrm{C}+\mathrm{NO}_{2} \rightarrow \mathrm{CO}+\mathrm{NO}
\end{gathered}
$$

and, in terms of 1-step heterogeneous reaction $[122,133]$ :

$$
\mathrm{C}+\left(2-g_{\mathrm{CO}}\right) \mathrm{NO}_{2} \rightarrow g_{\mathrm{CO}} \mathrm{CO}+\left(1-g_{\mathrm{CO}}\right) \mathrm{CO}_{2}+\left(2-g_{\mathrm{CO}}\right) \mathrm{NO}
$$


The $C O$ selectivity is given by [137]:

$$
g_{C O}=\frac{1}{1+k_{N O_{2}} y_{N O_{2}}^{n 3} \exp \left(\frac{E_{f 3}}{R T_{w}}\right)}
$$

and the reaction rate constant is given by equation 3.49 :

$$
k_{N O_{2}}=A_{N O_{2}} T_{w}^{n} \exp \left(-\frac{E_{N O_{2}}}{R T_{w}}\right)
$$

Particulate matter oxidation in the cake layer strongly depends upon the availability of $\mathrm{O}_{2}$ and $\mathrm{NO}_{2}$ at a given position in the layer itself. Following the approach developed by Bisset [108] and assuming that transport takes place mainly due to convection rather than diffusion, the $\mathrm{O}_{2}$ and $\mathrm{NO}_{2}$ mass fractions are conserved across the layer according to:

$$
\begin{gathered}
\frac{\partial}{\partial x}\left(\rho_{w} v_{w} Y_{O_{2}}\right)=-S_{p} \rho_{w} Y_{O_{2}} k_{t h, O_{2}}\left(1-\frac{f_{C O}}{2}\right) \\
\frac{\partial}{\partial x}\left(\rho_{w} v_{w} Y_{N O_{2}}\right)=-S_{p} \rho_{w} Y_{N O_{2}} k_{t h, N O_{2}}\left(2-g_{C O}\right)
\end{gathered}
$$

where $Y_{\mathrm{O}_{2}}$ and $\mathrm{Y}_{\mathrm{NO}_{2}}$ denote the mass fractions of $\mathrm{O}_{2}$ and $\mathrm{NO}_{2}$ entering the cake layer and that can be assumed to be equal to the values measured at the filter inlet, $v_{w}$ indicates the exhaust gas velocity approaching the soot layer and $\rho_{w}$ represents the exhaust density.

The reactive surface area of diesel PM $\left(S_{p}\right)$ can be expressed as:

$$
S_{p}=A_{p} \rho_{\text {soot }, \text { cake }}
$$

Temperature difference between gas and solid phase may be considered negligible according to previous studies [130], hence both gas density and velocity can be treated as 
constants allowing for simple integration of equations 3.50 and 3.51 which leads to the expressions for $\mathrm{O}_{2}$ and $\mathrm{NO}_{2}$ depletion rates:

$$
\begin{gathered}
R_{O_{2}, \text { cake }}=\rho_{w} v_{w} Y_{O_{2}, \text { cake }}\left[1-\exp \left(\frac{-S_{p} k_{t h, O_{2}}\left(1-\frac{f_{C O}}{2}\right) w_{\text {cake }}}{v_{w}}\right)\right] \\
R_{N O_{2}, \text { cake }}=\rho_{w} v_{w} Y_{N_{2}, \text { cake }}\left[1-\exp \left(\frac{-S_{p} k_{t h, N O_{2}}\left(2-g_{C O}\right) w_{\text {cake }}}{v_{w}}\right)\right]
\end{gathered}
$$

Knowledge of the depletion rates enables for the computation of the rate of consumption of the cake layer as:

$$
\frac{\partial}{\partial t}\left(\rho_{\text {soot }, \text { cake }} w_{\text {cake }}\right)=-\left[\frac{M_{c}}{M_{\mathrm{O}_{2}}}\left(\frac{R_{\mathrm{O}_{2}, \text { cake }}}{1-\frac{f C O}{2}}\right)+\frac{M_{c}}{M_{\mathrm{NO}_{2}}}\left(\frac{R_{\mathrm{NO}_{2}, \text { cake }}}{2-g_{C O}}\right)\right]
$$

\subsubsection{PM Catalytic Oxidation Kinetics: Washcoat Layer}

In case of catalytic coated substrates, the oxidation of PM particles should include also the reaction pathway due to $\mathrm{O}_{2}$ catalytic oxidation. The adoption of a recipe of noble metals on the surface of the substrate, in fact, helps to lower the activation temperature for the oxidation reaction due to oxygen. Hence, PM trapped in the washcoat layer is initially oxidized by catalytic means using the available $\mathrm{O}_{2}$ that leaves the cake layer and then is thermally oxidized using the remaining fraction of oxygen. This model has been initially developed by Konstandopoulos [106] who introduced the soot oxidation fraction $\beta$.

The 1-step heterogeneous reaction due to catalytic means is modeled similarly to the non-catalyzed reaction of $\mathrm{O}_{2}$, thus assuming PM soot to be purely carbonaceous: 


$$
C+\left(1-\frac{f_{C O}^{\prime}}{2}\right) O_{2} \rightarrow f_{C O}^{\prime} C O+\left(1-f_{C O}^{\prime}\right) C O_{2}
$$

where the catalytic $\mathrm{CO}$ selectivity is of the form:

$$
f_{C O}^{\prime}=\frac{1}{1+k_{\text {cat }} y_{O_{2}}^{n 2} \exp \left(\frac{E_{f 2}}{R T_{w}}\right)}
$$

The reaction rate constant $k_{\text {cat }}$ is assumed again to be of the Arrhenius form:

$$
k_{c a t}=A_{c a t} T_{w}^{n} \exp \left(\frac{-E_{c a t, O_{2}}}{R T_{w}}\right)
$$

Using the oxidation fraction $\beta, \mathrm{PM}$ can be assumed to be catalytically oxidized by a fraction $\beta$ of the available oxygen and then thermally oxidized by the remaining $(1-\beta)$ portion. Hence, the oxygen mass balance across the washcoat layer can be modeled as:

$$
\frac{\partial}{\partial x}\left(\rho_{w} v_{w} Y_{O 2, w s h}\right)=-S_{p} \rho_{w} Y_{O_{2}, w s h}\left[k_{t h, O_{2}}\left(1-\frac{f_{C O}}{2}\right)(1-\beta)+k_{c a t}\left(1-\frac{f_{C O}^{\prime}}{2}\right) \beta\right]
$$

Integration of the differential $\mathrm{O}_{2}$ mass balance across the washcoat layer thickness leads to the total oxygen depletion rate:

$$
R_{O_{2}, t o t}=\rho_{w} v_{w} Y_{O_{2}, w s h}\left[1-\exp \left(\frac{-S_{p} k^{*} w_{w s h}}{v_{w}}\right)\right]
$$

where:

$$
k^{*}=k_{t h}\left(1-\frac{f_{C O}}{2}\right)(1-\beta)+k_{c a t}\left(1-\frac{f_{C O}^{\prime}}{2}\right) \beta
$$

The catalytic and thermal fractions of the total oxygen depletion rate can then be computed applying the following: 


$$
\begin{gathered}
R_{O_{2}, t h}=R_{O_{2}, t o t}\left[\frac{k_{t h}\left(1-\frac{f_{C O}}{2}(1-\beta)\right)}{k^{*}}\right] \\
R_{O_{2}, c a t}=R_{O_{2}, t o t}\left[\frac{k_{c a t}\left(1-\frac{f_{C O}^{\prime}}{2} \beta\right)}{k^{*}}\right]
\end{gathered}
$$

and the $\mathrm{O}_{2}$ mass fraction leaving the washcoat layer and entering the wall thickness has the form of:

$$
Y_{O_{2}, w a l l}=Y_{O_{2}, w s h} \exp \left(\frac{-S_{p} k^{*} w_{w} s h}{v_{w}}\right)
$$

Concurrently to $\mathrm{O}_{2}$ catalytic and thermal oxidation, PM soot undergoes oxidation due to $\mathrm{NO}_{2}$ similarly to what already presented for the cake layer. The mass balance across the washcoat layer can be expressed as:

$$
\frac{\partial}{\partial x}\left(\rho_{w} v_{w} Y_{N_{2}, w s h}\right)=-S_{p} \rho_{w} Y_{N_{2}, w s h} k_{N O_{2}}\left(2-g_{C O}\right)
$$

and assuming isothermal conditions between washcoat layer and substrate wall, integration of equation 3.65 leads to the expressions for the $\mathrm{NO}_{2}$ depletion rate in the washcoat and the mass fraction entering the filter wall:

$$
\begin{gathered}
R_{\mathrm{NO}_{2}, w s h}=\rho_{w} v_{w} Y_{\mathrm{NO}_{2}, w s h}\left[1-\exp \left(\frac{-S_{p} k_{N O_{2}}\left(2-g_{C O}\right) w_{w s h}}{v_{w}}\right)\right] \\
Y_{\mathrm{NO}_{2}, \text { wall }}=Y_{\mathrm{NO}_{2}, w s h} \exp \left(\frac{-S_{p} k_{\mathrm{NO}_{2}}\left(2-g_{C O}\right) w_{w s h}}{v_{w}}\right)
\end{gathered}
$$

At last, the time rate of change for the washcoat layer can be computed taking into account all the reaction pathways described above: 


$$
\begin{aligned}
\frac{\partial}{\partial t}\left(\rho_{\text {soot }, w s h} w_{w s h}\right) & = \\
& -\left[\frac{M_{c}}{M_{O_{2}}}\left(\frac{R_{O_{2}, t h_{w s h}}}{1-\frac{f_{C O}}{2}}\right)+\frac{M_{c}}{M_{O_{2}}}\left(\frac{R_{O_{2}, c a t_{w s h}}}{1-\frac{f_{C O}^{\prime}}{2}}\right)\right] \\
& -\left[\frac{M_{c}}{M_{N O_{2}}}\left(\frac{R_{N O_{2}, w s h}}{2-g_{C O}}\right)\right]
\end{aligned}
$$

\subsubsection{Oxidation of $\mathrm{NO}$ into $\mathrm{NO}_{2}$ in the Washcoat Layer}

In case of particulate filters coated with catalytic material, the regeneration process due to continuous oxidation of PM by $\mathrm{NO}_{2}$ strongly depends upon the availability of the oxidizer at the inlet of filter. In general, engine-out $N O_{x}$ emissions from a modern heavy duty diesel engine are characterized by high values of the $\mathrm{NO} / \mathrm{NO}_{2}$ ratio, meaning that most ( $>85 \%$ ) of the $N O_{x}$ emissions are formed by nitric oxide. In order to guarantee a reasonable amount of $\mathrm{NO}_{2}$ at the filter inlet, engine and aftertreatment manufacturers usually couple the coated particulate filter with a Diesel Oxidation Catalyst placed upstream of the PM trap. This approach allows for simultaneous reduction of some of the regulated emissions $\left(\mathrm{CO}\right.$ and $\mathrm{HC}$ ) and for an increase in $\mathrm{NO}_{2}$ concentrations via $\mathrm{NO}$ oxidation.

However, as recent studies [138, 139] have discussed, a second mechanism for the production of $\mathrm{NO}_{2}$ takes place within the catalyzed PM filter. In fact, when the filter substrate is catalyzed with appropriate elements, a catalyzed oxidation of $\mathrm{NO}$ into $\mathrm{NO}_{2}$ may take place within the washcoat layer as the exhaust gas flows through leading to an increase in the net amount of $\mathrm{NO}_{2}$ available for soot oxidation. Konstandopoulos $[106,107]$ presented an approach to model this condition and these works have been then extended by Mohammed [135] who assumed re-oxidation of $N O$ to take place in the washcoat layer where the reaction can be facilitated by catalytic means.

The kinetic expression adopted to model such reaction is given by: 
Table 3.1: $\mathrm{O}_{2}$ and $\mathrm{NO}_{2}$ exponents.

\begin{tabular}{lrl}
\hline \hline Exponent & Value & \\
\hline $\mathrm{y}$ & 0.22 & \\
$\mathrm{n}$ & 0.5 & (Low Space Velocity) \\
$\mathrm{n}$ & 1.4 & (High Space Velocity) \\
\hline \hline
\end{tabular}

$$
\frac{d y_{N O_{2}}}{d t}=k_{N O} y_{O_{2}}^{\gamma} y_{N O}^{n}
$$

where the exponents for $\mathrm{O}_{2}$ and $\mathrm{NO}_{2}$ molar concentration are given in Table 3.1 according to what suggested in [135]. The reaction constant $k_{\mathrm{NO}_{2}}$, accordingly to the approach followed so far, is assumed to be of the Arrhenius form and expressed as:

$$
k_{N O}=A_{N O} T_{w}^{n} \exp \left(\frac{-E_{N O}}{R T_{w}}\right)
$$

where the pre-exponential constant $A_{N O}$ is used as tuning parameter to fit experimental data while the activation energy $E_{N O}$ has been considered equal to $9.06 E+4(k J / k m o l)$ according to what suggested in [106] and [121].

As the exhaust gas flows through the washcoat layer, $N O$ is eventually oxidized to form $\mathrm{NO}_{2}$ therefore the concentrations of $\mathrm{NO}, \mathrm{NO}_{2}$ and $\mathrm{O}_{2}$ are not constant across the layer thickness. This fact may lead to diffusive transport effects of the cited components across the layer due to the resulting gradients in concentratios. However, consistently with the oxidation theory applied to washcoat and cake layer, intra-layer diffusive effects are disregarded considering the limited thickness of the washcoat layer and the consequent short exhaust residence time. Moreover, changes in oxygen concentrations can be retained to be negligible due to the fact that $\mathrm{O}_{2}$ concentrations in diesel exhaust are usually orders of magnitude higher than those of $\mathrm{NO}$ and $\mathrm{NO}_{2}$. 
The model developed by Mohammed [135] assumes the washcoat layer to be divided in finite sub-layers. Under the quasi steady-state assumption, oxidation of PM particles is assumed to take place within each sub-layer while the re-oxidation of $\mathrm{NO}$ into $\mathrm{NO}_{2}$ is allowed between the sub-layers. The oxidation of PM mass trapped in the washcoat layer is modeled using the same theory presented in the previous section being the only difference confined to the integration domain of the differential equations. In this case, in fact, governing equations are integrated over the thickness of each sub-layer leading to the following expressions for the depletion rates:

$$
\begin{gathered}
R_{O_{2}, t o t, s u b_{i}}=\rho_{w} v_{w} Y_{O_{2}, s u b_{i-1}}\left[1-\exp \left(\frac{-S_{p} k^{*} w_{s u b_{i}}}{v_{w}}\right)\right] \\
R_{O_{2}, t h, s u b_{i}}=R_{O_{2}, t h, s u b_{i}}\left[\frac{k_{t h}\left(1-\frac{f_{C O}}{2}\right)(1-\beta)}{k^{*}}\right] \\
R_{O_{2}, c a t, s u b_{i}}=R_{O_{2}, c a t, s u b_{i}}\left[\frac{k_{c a t}\left(1-\frac{f_{C O}^{\prime}}{2}\right) \beta}{k^{*}}\right] \\
R_{N_{2}, s_{u b}}=\rho_{w} v_{w} Y_{N O_{2}, s u b_{i-1}}\left[1-\exp \left(\frac{-S_{p} k_{N O_{2}}\left(2-g_{C O}\right) w_{s u b_{i}}}{v_{w}}\right)\right]
\end{gathered}
$$

where $k^{*}$ is still expressed by equation 3.61 .

Due to the presence of the catalytic coating, $\mathrm{NO}$ eventually re-oxidize to give $\mathrm{NO}_{2}$ following the reaction path described above. This conversion is assumed to take place between the virtual sub-layers. In order to apply equation 3.69, the molar concentration between each pair of sub-layers is needed. An estimate for the needed concentration can be computed by applying a nitrogen atoms balance, as suggested by [140]:

$$
y_{\mathrm{NO}, \mathrm{sub}_{i}}+y_{\mathrm{NO}_{2}, \mathrm{sub}_{i}}=y_{\mathrm{NO}, \mathrm{sub}_{i-1}}+y_{\mathrm{NO}_{2}, \mathrm{sub}_{i-1}}
$$

Once the molar concentration has been computed, re-oxidation of $N O$ can be modeled by applying a first order finite difference scheme to equation 3.69 and the computation 
can be extended until the whole washcoat layer has considered. Since each sub-layer eventually is oxidized with a rate proportional to the net $\mathrm{NO}_{2}$ concentration available at each level, the equation representing the depletion of the whole washcoat layer (equation3.68) needs to be applied to each sub-layer. Hence, the total depletion can be obtained by adding together all the contributions given by the single sub-layers:

$$
w_{w s h}^{\text {depleted }}=\sum_{i=1}^{i=n} w_{i}^{\text {depleted }}
$$

\subsection{Energy Sub-model}

Filtration properties and oxidation kinetics presented in the previous sections show a strong dependency upon the filter temperature. Particle diffusivity, exhaust viscosity and reactions activation energies all require a reliable estimate for the filter wall temperature. Hence, a correct estimation of the energy balance for the whole DPF is mandatory if a reliable simulation of these processes has to be performed. Considering the 1-D nature of the model presented in this work, the framework developed by Kladopoulou et al. [62] has been adopted as main reference.

Assuming the temperature of the exhaust exiting the DPF to be equal to the substrate temperature within the filter, the energy balance for the solid phase becomes the only governing equation of interest:

$$
\left(m_{\text {soot }} c_{p, \text { soot }}+\rho_{\text {wall }} c_{p, \text { wall }} V_{\text {wall }}\right) \frac{d T_{w}}{d t}=\dot{Q}_{o x}+\dot{m}_{g} c_{p, g}\left(T_{g, \text { in }}-T_{w}\right)-\dot{Q}_{\text {loss }}
$$

where $\rho_{\text {wall }}$ denotes the bulk density of the substrate wall and may computed, according to what discussed in [62], considering the density of the ceramic material and the porosity of the filter: 


$$
\rho_{\text {wall }}=\rho_{\text {material }}\left(1-\varepsilon_{0}\right)
$$

while the volume of the solid fraction of the filter can be estimated as:

$$
V_{\text {substrate }}=V_{D P F}-V_{\text {Channels }}=\frac{\pi D_{f}^{2}}{4} L-\alpha^{2} N_{\text {cell }} L
$$

Heat losses to the environment have been simulated by implementation of heat losses through the DPF packaging and by convection to the surroundings:

$$
Q_{\text {loss }}=\frac{T-T_{e n v}}{R_{t}}
$$

where $T$ is the temperature of the filter, $T_{e} n v$ is the temperature of the environment and $R_{t}$ is the overall thermal resistance across the insulation material, metal can and convective heat transfer coefficient. The overall thermal resistance can be computed by $[62]$ :

$$
R_{t}=\frac{\ln \left(r_{i n s} / r_{f}\right)}{2 \pi k_{i n s} L}+\frac{\ln \left(r_{c} / r_{i n s}\right)}{2 \pi k_{c} L}+\frac{1}{h 2 \pi r_{c} L}
$$

where $r_{i n s}, r_{f}$ and $r_{c}$ are outer radius of insulation layer, filter radius and outer radius of canister. The thermal conductivity between filter and insulation is denoted with $k_{\text {ins }}$ and the thermal conductivity between insulation and metal canister is represented by $r_{c}$.

The convective heat transfer $h$ is computed according to Bejan [141] using the correlations for the case of natural convection over a horizontal cylinder with uniform surface temperature. This particular case was selected to replicate the experimental conditions. In the cited case, the Nusselt number can be expressed as:

$$
N U_{D}=\left[0.6+\frac{0.387 R a_{d}^{1 / 6}}{\left(1+\left(\frac{0.559}{P r}\right)^{9 / 16}\right)^{8 / 27}}\right]^{2}
$$


where $\operatorname{Pr}$ is the Prandtl number and $R a$ denotes the Rayleigh number which can be computed as follows:

$$
R a_{D}=\frac{g \beta\left(T_{w}-T_{e n v}\right) D^{3}}{\alpha \nu}
$$

In the equation above, $g$ denotes the gravitational acceleration, $\beta$ indicates the coefficient of thermal expansion, $\alpha$ the thermal diffusivity and $\nu$ the kinematic viscosity of the surrounding air.

The rate of heat production due to PM soot oxidation can be computed as [132]:

$$
\begin{aligned}
\dot{Q}_{O x}= & \frac{\Delta H^{t h}}{M_{\mathrm{O}_{2}}}\left(\frac{R_{\mathrm{O}_{2}, c k+w s h}^{t h}}{1-f_{C O} / 2}\right)+\frac{\Delta H^{c a t}}{M_{\mathrm{O}_{2}}}\left(\frac{R_{\mathrm{O}_{2}, w s h}^{c a t}}{1-f_{C O}^{\prime} / 2}\right) \\
& +\frac{\Delta H^{N O_{2}}}{M_{N O_{2}}}\left(\frac{R_{\mathrm{NO}_{2}, c k+w s h}}{2-g_{C O}}\right)
\end{aligned}
$$

where the reaction rate are given according to:

$$
\begin{gathered}
\Delta H^{t h}=f_{C O} \Delta H_{C O}+\left(1-f_{C O}\right) \Delta H_{C O_{2}} \\
\Delta H^{c a t}=f_{C O}^{\prime} \Delta H_{C O}+\left(1-f_{C O}^{\prime}\right) \Delta H_{C O_{2}} \\
\Delta H^{N O_{2}}=g_{C O} \Delta H_{C O}+\left(2-g_{C O}\right) \Delta H_{C O_{2}}
\end{gathered}
$$

and the terms $\Delta H_{\text {compound }}$ can be acquired as enthalpy of formation for the specific compound.

\subsection{Pressure Drop Sub-model}

The model used to estimate the pressure drop across the particulate filter is the one initially developed by Konstandopoulos in [111] for a clean filter and later extended to the case of loaded filter by the same author in [57]. The basic equation for the pressure 


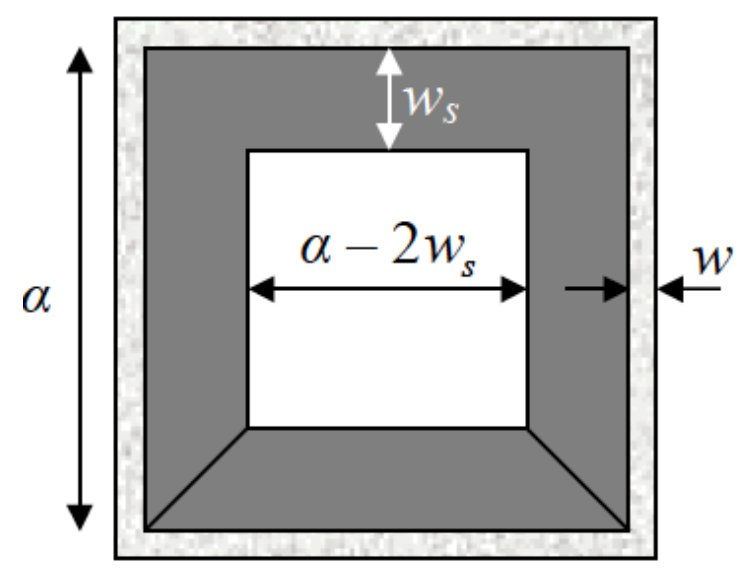

Figure 3.7: Channel geometry, adopted from [57]

drop across a clean filter can be expressed as:

$$
\Delta P_{\text {clean }}=\frac{\mu}{k_{0}} \frac{U \alpha}{4 L} w+\frac{2 \mu F}{3 \alpha^{2}} U L a
$$

In the expression above the first terms represents the pressure drop across the substrate wall of thickness $w$ and permeability $k_{0}$ while the second term takes into account the pressure along the filter channels of width $a$ and length $L$. Following the approach presented in [62], equation 3.88 can be rewritten as:

$$
\Delta P=\frac{\mu Q}{V_{\text {trap }}}(\alpha+w)^{2}\left(\frac{w}{k_{0} \alpha}+\frac{4 F L^{2}}{3 \alpha^{4}}+\frac{4 F L^{2}}{3 \alpha^{4}}\right)
$$

In case of a loaded filter, the pressure drop across the particulate trap can be expressed as composition of four main contributions:

$$
\Delta P=\Delta P_{\text {wall }}+\Delta P_{\text {soot-layer }}+\Delta P_{\text {InletCh. }}+\Delta P_{\text {OutletCh }}
$$

With reference to Figure 3.7, the first two terms can be expressed as:

$$
\begin{gathered}
\Delta P_{\text {wall }}=\frac{\mu Q}{2 V_{\text {trap }}}(\alpha+w)^{2}\left(\frac{w}{k_{0} \alpha}\right) \\
\Delta P_{\text {soot-layer }}=\frac{\mu Q(\alpha+w)^{2}}{L \pi D_{f}^{2} k_{\text {soot }}} \ln \left(\frac{\alpha}{\alpha-2 w_{s}}\right)
\end{gathered}
$$


where, $Q$ denotes the exhaust volumetric flow rate while $k_{\text {soot }}$ and $w_{s}$ represent permeability and thickness of the soot layer respectively.

The amount of PM mass deposited as soot layer on top of the substrate wall can be expressed as:

$$
V_{\text {soot }}=\frac{m_{c}}{N_{\text {cells }} \rho_{\text {soot }, \text { ck }}}=4 \frac{\alpha+\left(\alpha-2 w_{s}\right)}{2} w_{s} L
$$

from where it is possible to obtain the relationship for the soot layer thickness (equation $3.94)$.

$$
w_{s}=\frac{\alpha-\sqrt{\alpha^{2}-\frac{m_{c}}{N_{\text {cells }} L \rho_{\text {soot }, c k}}}}{2}
$$

As PM mass is collected on the surface of the wall, the hydraulic diameter of the inlet channels shrink due to the growth of the soot layer while the outlet channels retain their initial size as all PM particles are assumed to be collected in the filter wall and soot layer. Hence, the pressure drop due to flow friction is given by:

$$
\begin{gathered}
\Delta P_{\text {InletCh. }}=\frac{\mu Q}{2 V_{\text {trap }}}(\alpha+w)^{2}\left(\frac{4 F L^{2}}{3\left(\alpha-2 w_{s}\right)^{4}}\right) \\
\Delta P_{\text {OutletCh. }}=\frac{\mu Q}{2 V_{\text {trap }}}(\alpha+w)^{2}\left(\frac{4 F L^{2}}{3 \alpha^{4}}\right)
\end{gathered}
$$




\section{Chapter 4}

\section{Experimental Setup}

Calibration and validation of the DPF model presented in the previous sections have been performed at the Engine and Emissions Research Laboratory (EERL) of West Virginia University via comparison with experimental data gathered during engine dynamometer testing. Experimental procedures followed the recommendations outlined in the Code of Federal Regulation (CFR), Title 40, Part 1065 protocol.

\subsection{Engine and Aftertreatment System}

The engine selected for this study was a MY2004 Mack ${ }^{\circledR}$ MP7-355E 11 liters heavyduty diesel engine equipped with high-pressure loop Exhaust Gas Recirculation system (EGR) and a Variable Geometry Turbocharger (VGT). The engine was compliant with the US-EPA 2004 HD emission standards and it was calibrated to achieve compliancy without an OEM aftertreatment system. The diesel fuel used in this work was a commercially available ultra-low sulfur diesel (ULSD) with a maximum sulfur content of 15 ppm. Detailed information about the test engine are provided in Table 4.1.

Since the test engine was equipped with no aftertreatment, a "retrofit" approach had to be used. The aftertreatment system that was selected for this study was a Johnson Matthey Continuously Regenerating Trap (C-CRT $\left.{ }^{\circledR}\right)$ (Figure 4.1) which uses a diesel oxidation catalyst in front of the filter to generate the $\mathrm{NO}_{2}$ required to oxidize the collected 
Table 4.1: Test Engine Specifications.

\begin{tabular}{ll}
\hline \hline Model & \multicolumn{1}{c}{ Mack $^{\circledR}$ MP7-355E } \\
\hline Configuration & 6 Cylinders, In-line \\
Aspiration & Sliding Nozzle VGT with Intercooler \\
Injection System & Dual Solenoid Electronic Unit Injector (EUI) \\
Displacement, L (cu-in) & $11(659)$ \\
Compression Ratio & $16: 1$ \\
Bore $\times$ Stroke, mm (in) & $122.94 \times 151.89(4.84 \times 5.98)$ \\
Rated Torque, Nm (ft-lb) & $1844(1360) @ 1200 \mathrm{RPM}$ \\
Rated Power, kW (bhp) & $265(355) @ 1800 \mathrm{RPM}$ \\
\hline \hline
\end{tabular}

PM mass. This fact makes this system a suitable candidate for retrofit applications as no integration with engine controls and calibration is needed. Further, in order to improve conversion efficiency at lower temperatures, the C-CRT technology extends the catalytic coating also to the substrate wall of the filter itself.

These characteristics made the C-CRT a suitable system for the purposes of this study since the DPF model included a passive regeneration sub-routine to account for soot oxidation due to $\mathrm{NO}_{2}$. Manufacturer specifications for the C-CRT system are reported in Table 4.2 .

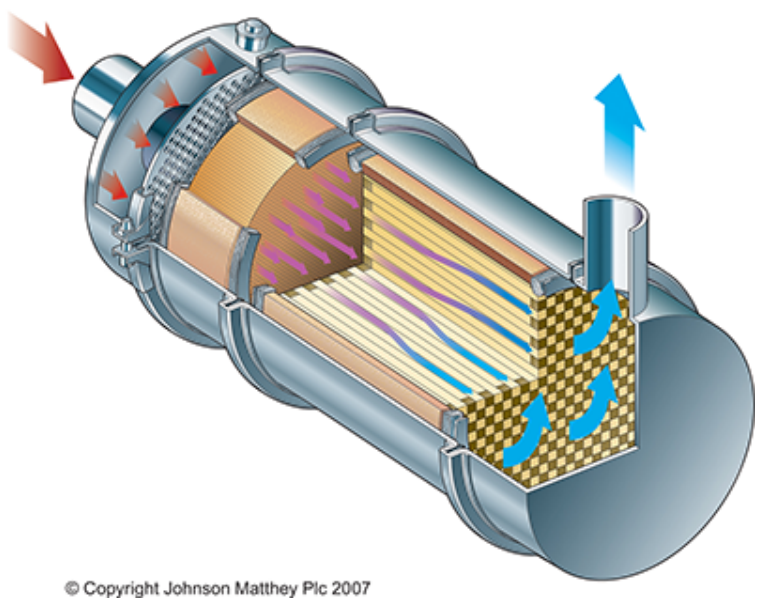

Figure 4.1: Johnson Matthey C-CRT. 


\subsection{Instrumentation and Laboratory Setup}

Regulated gaseous emissions downstream of the C-CRT were sampled diluted using a subsonic venturi constant volume sampling system (CSV-SSV) and measured by a Horiba ${ }^{\circledR}$ MEXA 7200D analytical system. Gaseous emissions measured by the Horiba system comprised: carbon monoxide $(\mathrm{CO})$ and carbon dioxide $\left(\mathrm{CO}_{2}\right)$ via the non-dispersive (NDIR) detection method, nitric oxide $(N O)$ and total oxide of nitrogen $\left(N O_{x}\right)$ using the Chemi-luminescence (CLD) method, total hydrocarbons $(\mathrm{THC})$ and methane $\left(\mathrm{CH}_{4}\right)$ using a heated flame ionization detector (HFID) having a second channel equipped with a methane cutter.

Particulate matter emissions where measured via the gravimetric method: diluted exhaust gas was drawn from the full flow dilution tunnel through a secondary dilution system, and then sampled onto a Pallflex $47 \mathrm{~mm}$ Teflo filter. Sampled filters were weighted in an environmentally controlled clean room (Class 1000) using a Sartorius microbalance with an accuracy of $0.1 \mu g$.

Concurrently with diluted emissions, raw emissions were measured at three different locations: upstream the C-CRT, between the DOC and the DPF and downstream of the C-CRT. The instrumentation comprised: a Horiba non-dispersive infrared (NDIR) $\mathrm{CO}_{2}$ analyzer, Rosemount ${ }^{\circledR} 402$ heated flame ionization detector (HFID) HC analyzer and a California Analytical Instrument ${ }^{\circledR}$ CAI 600 HCLD-C $N O_{x}$ analyzer. Solid fraction of

Table 4.2: Johnson Matthey C-CRT Specifications.

\begin{tabular}{lcc}
\hline \hline Parameter & DOC & DPF \\
\hline Diameter (in) & 12 & 12 \\
Length (in) & 5 & 12 \\
Cell Density (cpsi) & 400 & 100 \\
Wall Thickness (mil) & 4 & 12 \\
Clean Wall Porosity & - & 0.5 \\
\hline \hline
\end{tabular}


particulate matter in the raw exhaust was measured upstream the CCRT by using an AVL-483 Micro Soot Sensor whilst characterization of PM particles size was performed by employing a TSI Engine Exhaust Particle Spectrometer (EEPS). In order to bring particle count concentrations within acceptable levels for the instrument, a double stage partial-flow sampling system was adopted and installed upstream of the C-CRT. The dilution process comprised a first stage dilution with hot air $\left(150^{\circ} \mathrm{C}\right)$ followed by a second stage with air at ambient temperature. A schematic of the experimental setup is given in Figure 4.3.

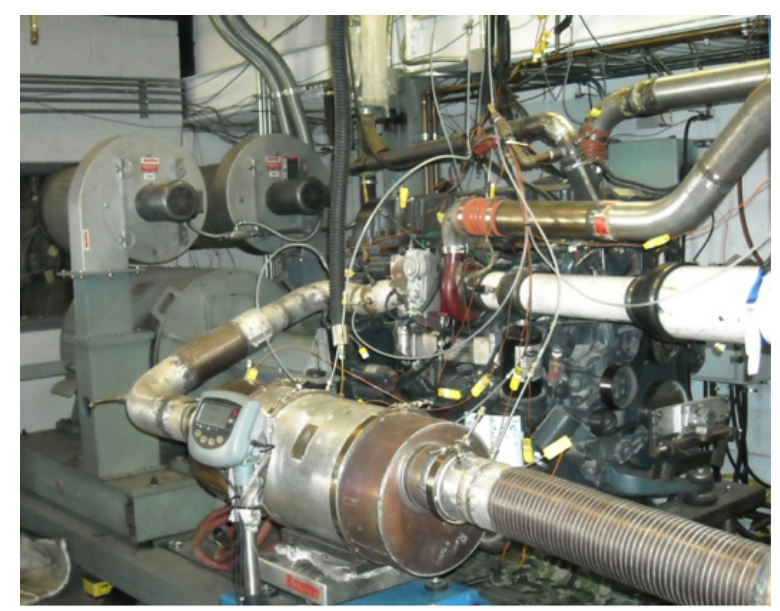

Figure 4.2: Test engine and C-CRT on engine dynamometer at WVU-EERL.

Further, the C-CRT was instrumented with temperature sensors and pressure transducers placed before the DOC, between the DOC and the DPF, and directly downstream of the DPF. The differential pressure across the filter was measured using a Validyne ${ }^{\circledR}$ P55D differential pressure transducer.

A weight scale was placed directly under the DPF-DOC system during the test; this allowed for quick determination of the system mass after detaching the exhaust pipes and removal of the thermocouple/pressure transducer harnessing following each test. The scale used for measuring the mass of the system was an Ohaus CD-11 with a resolution of 5 grams. The initial mass of the system was recorded to be $50.125 \mathrm{~kg}$. 


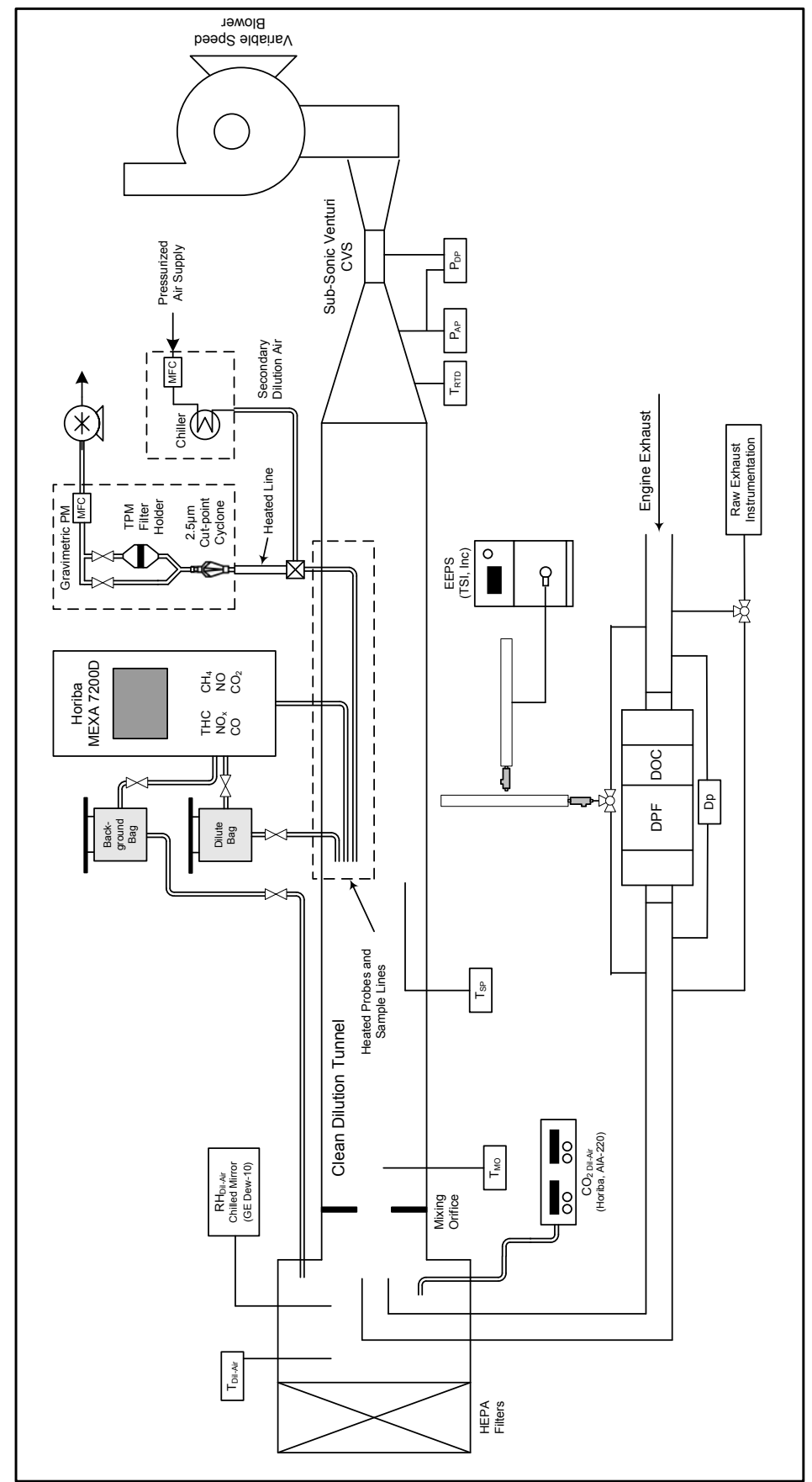

Figure 4.3: Laboratory setup at WVU-EERL.

\subsubsection{Test Matrix}

Selection of the engine modes for DPF loading and regeneration was based on preliminary testing, having the test engine exercised over the 13-Mode European Stationary Cycle (ESC). Since the C-CRT technology is optimized for continuous regeneration, an engine mode characterized by low exhaust temperature was needed in order to limit soot 
oxidation during the loading process. Initially, an engine mode characterized by $25 \%$ load and rated speed (R25) was selected as suitable for this application. However, the measured exhaust temperature $\left(280^{\circ} \mathrm{C}\right)$ appeared to be too high as the DPF reached the equilibrium point (no soot accumulation) after $4 \mathrm{hr}$ from the beginning of the test (left graph of Figure 4.5). This fact was verified by the trend of the differentail pressure measured across the DPF during additional $4 \mathrm{hr}$ of testing (right graph of Figure 4.5).

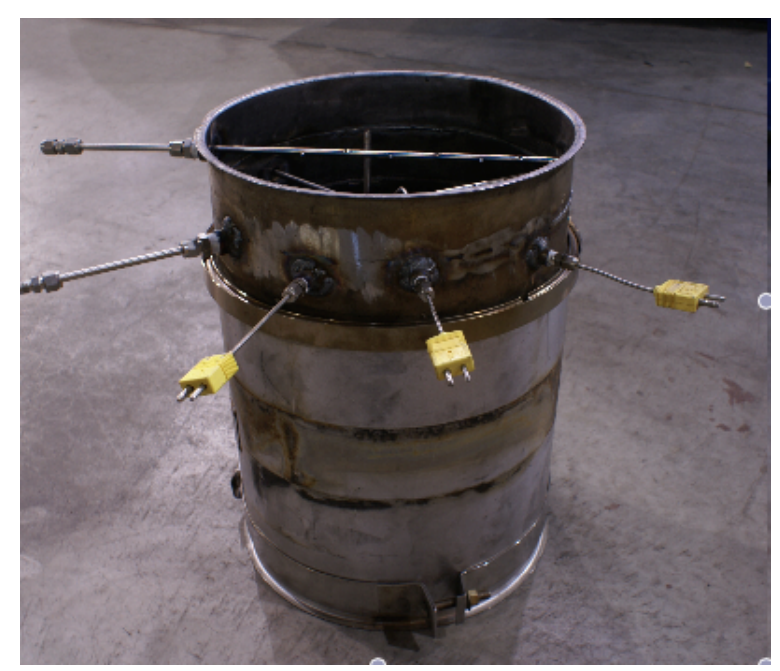

Figure 4.4: Instrumented C-CRT used during engine testing.
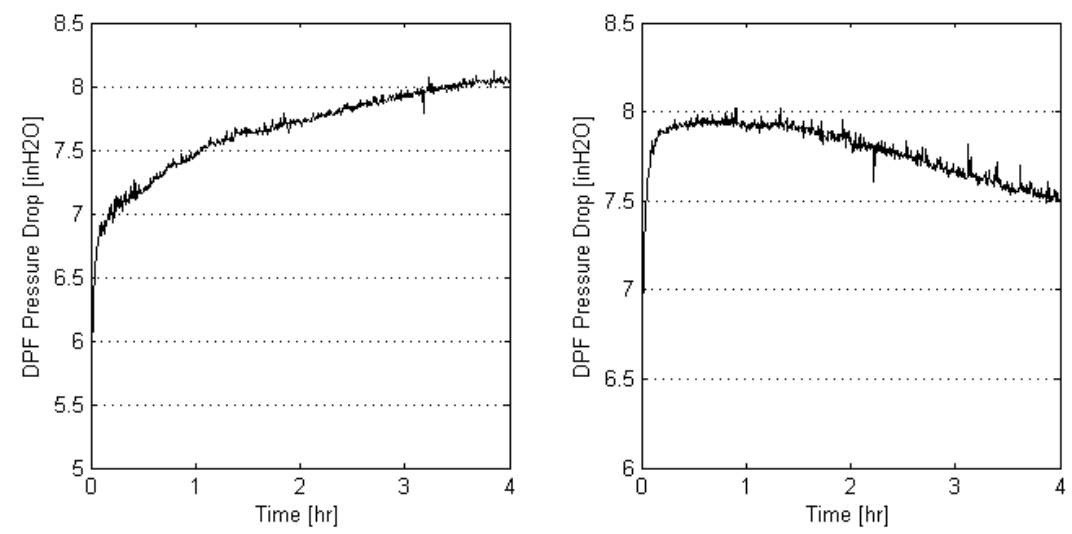

Figure 4.5: Pressure drop measured across the DPF during the R25 engine modes.

In order to guarantee a minimum soot loading within the C-CRT, the engine mode selected for the loading process was characterized by $10 \%$ engine load and rated speed (R10). This engine operation resulted in lower exhaust temperature and $N O_{x}$ emissions, and ultimately in a lower regeneration rate within the DPF. On the other hand, the 
engine mode selected for filter regeneration was characterized by both full engine load and speed (R100) in order to increase the exhaust temperature and enhance the regeneration process. Detailed information about the steady-state engine modes are provided in Table 4.3.

Table 4.3: Characteristics of the steady-state engine modes.

\begin{tabular}{lcc}
\hline \hline Engine Mode & R-10 & R-100 \\
\hline Engine Load [ft-lb] & 105 & 1018 \\
Engine Speed [rpm] & 1800 & 1800 \\
Duration [hr] & 25 & 2 \\
Fuel Consumption $[\mathrm{kg} / \mathrm{hr}]$ & 5.84 & 55.85 \\
CCRT Inlet Temp. $\left[{ }^{\circ} \mathrm{C}\right]$ & 226.5 & 483.8 \\
Exhaust Flow Rate $[\mathrm{scfm}]$ & 256.4 & 588.1 \\
\hline \hline
\end{tabular}

In order to verify the accuracy of the DPF model to track filter conditions during transient operations, the Federal Testing Procedure (FTP) was selected as the transient engine test cycle. The transient portion of the text matrix was performed between filter loading and filter regeneration in order to test the model in capturing passive regeneration with variable exhaust characteristics. In particular, the first half of the FTP cycle is characterized by exhaust temperatures in the range of $150^{\circ} \mathrm{C}$ while the last part of the test corresponds to exhaust temperatures in the order of $350^{\circ} \mathrm{C}$. Therefore, DPF loading is likely to occur during the first portion of the FTP while filter regeneration is more likely to take place over the last 10 minutes of the cycle.

\subsubsection{Experimental Procedure}

During the testing activity, the engine was exercised continuously for 27 hours as result of a text matrix which included loading, regeneration and transient cycles. Before the beginning of the test matrix, the engine was conducted at full load/speed for 1 hour. This preliminary test was needed in order to bring the C-CRT to a "reference level" that could be used as starting point for the loading cycle. In fact, the C-CRT used in this study was an aged system and no information regarding the current loading status were 
available. Therefore, a "forced" regeneration was simulated to limit the amount of PM trapped in the C-CRT before the beginning of the actual test matrix discussed earlier.

Once the C-CRT reached the reference status, the engine was exercised over the mode R10 for a total duration of $24 \mathrm{hr}$. The loading process was interrupted every $4 \mathrm{hr}$ to allow for DPF weighing and re-fueling. In order to maintain consistency with respect to engine and aftertreatment conditions, each pause was scheduled to last always 15 minutes. The weighing procedure was performed as follows: a) engine was shut down, b) sampling lines for raw exhaust emissions measurement as well as harness for thermocouples and pressure transducers were removed from the C-CRT, c) scale was placed underneath the CCRT, d) exhaust clamps on both sides of the C-CRT were removed and the system was placed onto the scale, and e) mass of the C-CRT was recorded. After the loading portion of the test matrix, the engine was conducted over two transient FTP cycles and finally over the R100 engine mode for $2 \mathrm{hr}$. 


\section{Chapter 5}

\section{Calibration Methodology}

One of the critical aspects of aftertreatment modeling is represented by the procedure followed to calibrate the tuning parameters in order to match simulated results with experimental data. This chapter discusses the methodologies followed to tune both DOC and DPF models.

\subsection{DPF Model}

\subsubsection{Virtual Conditioning}

Previous studies available in the literature aimed at the development of DPF models generally presents a correlation between simulated results and experimental data gathered using a new, not-aged experimental apparatus. The main goal of these works has been focused on tuning DPF models in order to capture the transition from deep bed filtration to cake deposition. However, as discussed by Koltsakis [142], this condition is lost quickly after a certain number of loading/regeneration cycles.

The correlation between soot load and pressure drop is deeply altered by the aging process of the filter; while in a clean, un-conditioned filter it is possible to appreciate the contribution of both deep bed filtration and cake deposition (non-linear pressure curve), this is not the case for a conditioned unit (Figure 1.5). As discussed by Rose et al. [56] 
the effect of soot loading on filter pressure drop arises from two contributions: i) increase of flow friction in the inlet channels and ii) increase of the substrate wall resistance. The increase in friction derives from the restriction of the inlet channels due to accumulation of PM mass whilst the increase in substrate wall resistance arises from the combination of two different phenomena. At first PM particles entering the wall during the deep bed filtration mechanism generate a drop in wall permeability which is associated with a steep increase in pressure drop. Then, as the wall becomes loaded with PM mass, a soot layer builds up on the surface of the wall and acts as an additional filter in series.

Although both deep bed and cake filtration mechanisms can be observed on a new, unconditioned filter (Figure 1.5), generally the impact of deep bed filtration has been found to be minimal during actual use of the filter either after few loading/regeneration cycles or once a small amount of ash has been accumulated [56, 143]; these conditions may lead to the formation of a thin membrane over the wall surface, preventing PM soot from penetrating the wall, and hence inhibiting the deep bed filtration mechanism responsible for most of the pressure drop across the filter.

The change in correlation between pressure drop and soot load for a fresh and a conditioned filter is therefore the result of a the cyclic process of loading and regeneration experienced by the filter and it is enhanced in case of catalyzed filters due to partial $\mathrm{NO}_{2}$ regenerations [56]. This fact is shown in Figure 5.1 which represents the conditioning process of a fresh filter. It is worth noting that as result of the aging process, same values of pressure drop can be achieved with different values of soot load.

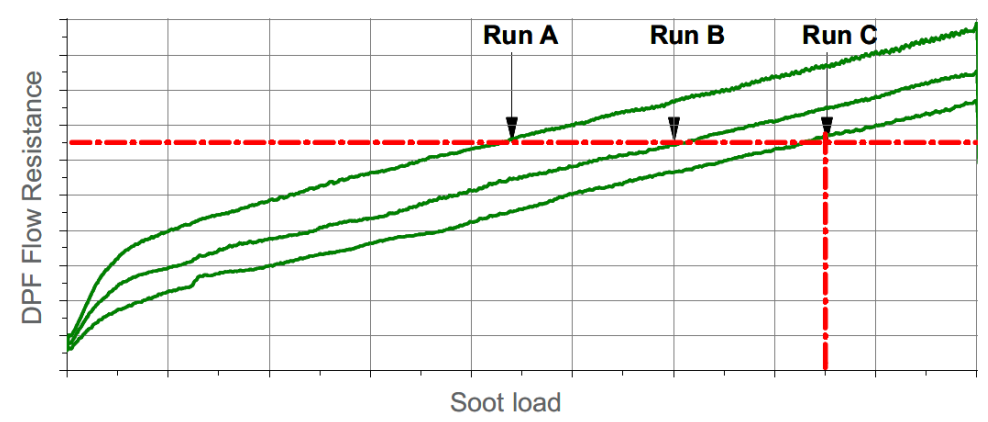

Figure 5.1: Effect of filter conditioning on pressure-soot load correlation [56]. 
In the given background, one of the main challenges encountered in this study was to replicate the behavior of an aftertreatment device with unknown loading/regeneration history. In order to correctly reproduce the behavior of the CCRT, coherent initial conditions had to be imposed to the model. To this aim a procedure called "virtual conditioning" was defined and included in the calibration process of the model parameters. Virtual conditioning allowed to replicate the aging of the real filter by simulating sequences of loading and regeneration cycles until a stabilization in filter backpressure was observed and simulated results matched measured values of the first R10 loading mode. The conditioning procedure was as follows: at first, "clean" filter settings were applied to the DPF model using manufacturer specifications and a loading process (R10) was simulated until a balance point $(d p / d t=0)$ was reached. At this point a regeneration cycle of 2 hrs was applied to the model and the procedure was repeated until no change in pressure drop curve was observed between two consecutive conditioning cycles.

Due to the virtual conditioning, substrate properties evolve from values which are characteristic of a clean filter to those of an aged system. At the beginning of the process, PM particles entering the DPF can easily reach the clean substrate due to the lack of a membrane on top of the filter wall. During this phase the microstructure of the wall changes leading to a drop in permeability and, as a result, an increase in filter pressure drop. The trend of the computed permeability is presented in Figure 5.2 which refers to the first $4 \mathrm{hr}$ of virtual conditioning.

The substrate permeability depicted in Figure 5.2 is presented as a function of the normalized wall thickness at different times during the loading process. At time $t=0$ permeability is constant over the wall thickness (clean filter); as the wall collects more PM particles the permeability is distributed over the wall thickness with areas characterized by more flow resistance (i.e. more soot load) towards the surface of the substrate.

As the porosity of the wall decreases due to accumulation of PM mass, the partition coefficient which controls the rate of cake deposition increases leading to a thicker cake 


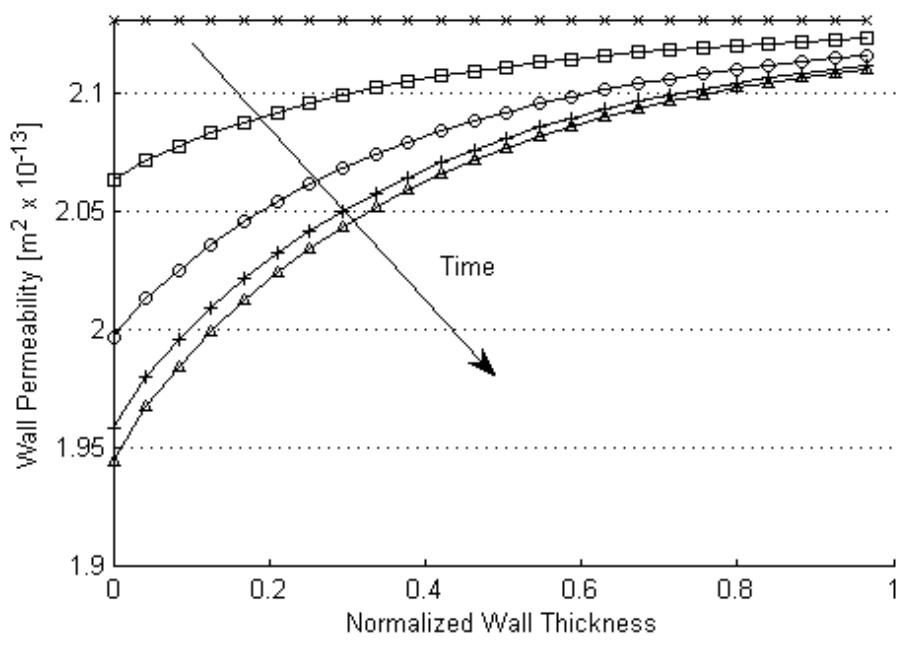

Figure 5.2: Evolution of substrate permeability during the initial phase of virtual conditioning.

layer and, as a result, to an increase of the cake collection efficiency.

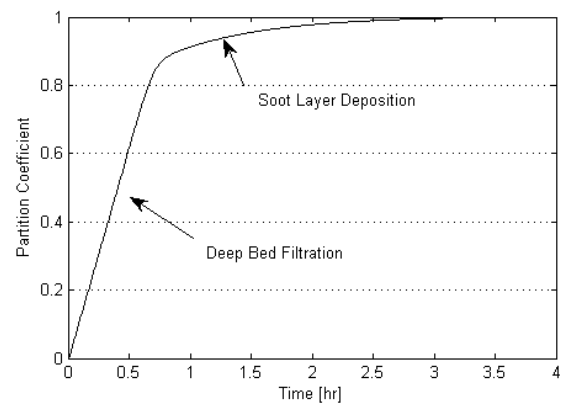

(a) Partition Coefficient

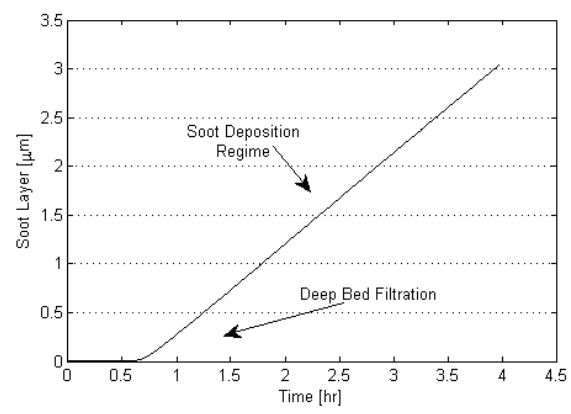

(b) Soot Layer

Figure 5.3: Evolution of Substrate Properties during Virtual Conditioning.

As the process of virtual aging continues the modeled filter eventually reaches a condition in which a small layer of soot is left on top of the substrate wall even after regeneration cycles (R100 mode); this layer limits the amount of PM mass that can reach the substrate wall leading to a condition in which most of the soot trapped in the filter is deposited as cake layer (Figure 5.4).

The last portion of the virtual conditioning process is depicted in Figure 5.5 which shows the simulated pressure drop during the last regeneration cycle of the virtual conditioning used to bring the virtual filter at the same reference status of the experimental 


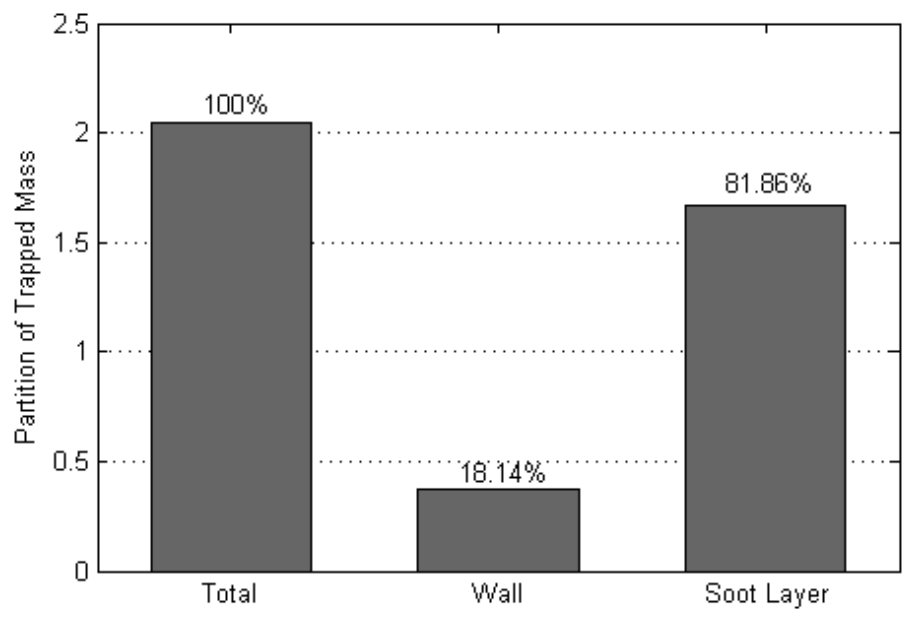

Figure 5.4: Distribution of the PM mass trapped within the DPF during the virtual conditioning.

apparatus followed by a comparison of the simulated and measured pressure drop signals recorded during the first R10 loading cycle. If measured and simulated pressure drop were in agreement the filter was considered to be correctly conditioned and the calibration process was started.

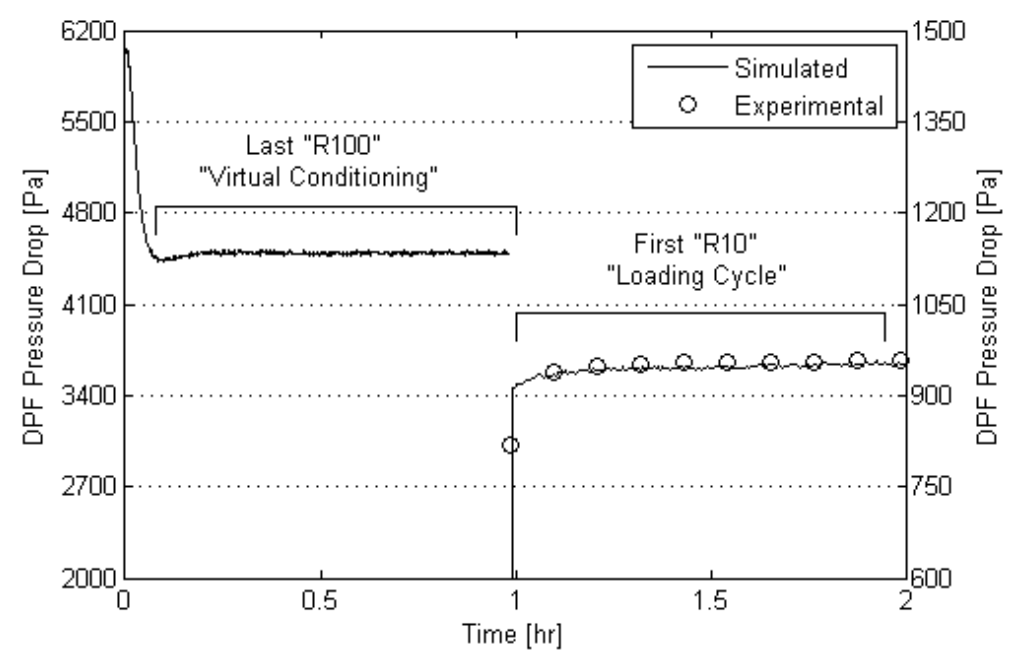

Figure 5.5: Filter pressure drop during last portion of virtual conditioning.

\subsubsection{Model Tuning}

The test matrix described in the previous chapter comprised 24 hrs of loading cycle, two FTP cycles and a final regeneration cycle with a duration of 1.5 hours. The loading cycle was divided in six portions of 4 hours each to allow for filter weighing and engine refueling. 
In order to obtain a strong calibration, capable of replicating different engine cycles with just minor adjustment to the tuning parameters a standard calibration methodology had to be improved. In fact, in most of the studies available in the open literature it is possible to observe that model calibrations are engine-mode dependent and no extensive work is presented in case of transient operations. This means that depending upon the particular steady-state cycle that needs to be simulated, key tuning parameters are adjusted to match the experimental data. The result of this calibration approach is a set of tuned parameters to be used depending upon particular operating condition. This methodology may fail when a combination or sequence of steady-state and transient cycles has to be simulated.

To overcome this limitation and generate a model calibration that allows for simulation of different operating conditions without a continuous refinement of the key values, a specific tuning procedure was defined and organized as follows: $i$ ) initial values for the tuning parameters and clean filter properties were selected, $i i)$ the virtual conditioning was performed following the procedure explained earlier, iii) the conditioned model was used to consecutively replicate experimental data of each engine cycle (6 R10 cycles, 2 FTPs, and 1 R100 cycle), and $i v$ if the $i-t h$ cycle was replicated correctly then the simulation was moved on to the following cycle until the entire set was reproduced, otherwise the tuning parameters were changed and the calibration process was started again (point $i$ ).

The adoption of such tuning procedure ensured that the obtained calibration was not dependent upon the particular engine mode as the key parameters were tuned in order to minimize the difference between simulated and measured data over a composition of different engine cycles (R10, R100 and FTP). This fact led to an improvement in calibration robustness allowing to simulate steady-state and transient cycles without any variation in key parameters. The set of values resulting from the tuning process is given in Table 5.1 while other DPF properties which were held constant during the calibration process are reported in Table 5.2. 
Table 5.1: DPF model calibrated parameters.

\begin{tabular}{llcc}
\hline \hline Symbol & Description & Value & Units \\
\hline$E_{t h, O_{2}}$ & $\mathrm{O}_{2}$ Thermal Activation Energy & $1.610^{4}$ & $\mathrm{~K}$ \\
$E_{c a t, \mathrm{O}_{2}}$ & $\mathrm{O}_{2}$ Catalytic Activation Energy & $1.410^{4}$ & $\mathrm{~K}$ \\
$E_{\mathrm{NO}_{2}}$ & $\mathrm{NO}_{2}$ Activation Energy & $1.1210^{4}$ & $\mathrm{~K}$ \\
$\rho_{\text {soot }, w}$ & Soot Packing Density in the Wall & 8 & $\mathrm{~kg} / \mathrm{m}^{3}$ \\
$\rho_{\text {soot }, c k}$ & Soot Packing Density in the Cake & 35 & $\mathrm{~kg} / \mathrm{m}^{3}$ \\
$k_{0}$ & Clean Wall Permeability & $2.1310^{-13}$ & $\mathrm{~m}^{2}$ \\
$k_{\text {soot }}$ & Soot Permeability & $3.210^{-14}$ & $\mathrm{~m}^{2}$ \\
$\psi$ & Wall Percolation Factor & 0.83 & - \\
\hline \hline
\end{tabular}

Table 5.2: DPF model parameters used in this work.

\begin{tabular}{llcc}
\hline \hline Symbol & Description & Value & Units \\
\hline$A_{t h}$ & $\mathrm{O}_{2}$ Thermal Rate Constant & 25.08 & $\mathrm{~m} / \mathrm{sk}$ \\
$A_{c a t}$ & $\mathrm{O}_{2}$ Catalytic Rate Constant & 2.84 & $\mathrm{~m} / \mathrm{sk}$ \\
$A_{\mathrm{NO}_{2}}$ & $\mathrm{NO}_{2}$ Catalytic Rate Constant & 2.9 & $\mathrm{~m} / \mathrm{sk}$ \\
$S_{p}$ & Soot Specific Area & $5.510^{7}$ & $1 / \mathrm{m}$ \\
$\epsilon_{0}$ & Porosity of Clean Filter & 0.5 & - \\
$\lambda_{D P F}$ & DPF Substrate Thermal Conductivity & 1.0 & $\mathrm{~W} / \mathrm{mK}$ \\
$c p_{w, D P F}$ & DPF Substrate Specific Heat & 1000 & $\mathrm{~J} / \mathrm{kg} \mathrm{K}$ \\
\hline \hline
\end{tabular}

\subsection{DOC Model}

One of the challenges associated with modeling of diesel oxidation catalyst lies in the development of a robust calibration. As discussed by Sampara [86], it is a common practice in the literature to use reaction rate forms suggested by Voltz et al. [117] and calibrate the rate constants using engine data.

The data needed to calibrate the rate constants can be either in the form of light-off curves or in form of conversion rates measured over an entire FTP engine cycle. The problem with using light-off data is that the data is confined to a narrow temperature window and refers to nominal inlet concentrations. This means that theoretically more than one light-curve is needed to cover several inlet concentration levels, gas temperature and space velocity. On the other hand, FTP data may contain little information to 
calibrate the rate constants and is often in the transport controlled regime.

Since the objective of this work is the development of a DOC+DPF model for OBD monitors development, a detailed analysis of the kinetics taking place within the DOC would be beyond the scope of the thesis. Instead, apparent values for kinetic rate constants and mass transport parameters can be approximated using a methodology similar to what presented by Voltz [117] and Sampara [86] and based on the minimization of an objective function.

Following the approach of Voltz [117], the sum of squares of the errors between experimental data and simulated values was selected as objective function reducing the calibration process to a non-linear least squares problem. A set of calibration parameters was obtained when minimization of the objective function was completed. The algorithm that was used for the minimization process was the Levenberg-Marquardt method for non-linear least squares.

As long as the conservation of energy is concerned, the parameters used for the optimization process included:

1. Constants $A_{N}$ and $B_{N}$ appearing in the correlation used for the Nusselt number (Equation 2.33).

2. Thermal conductivity of the substrate $\left(\lambda_{s}\right)$.

3. Density of the substrate $\left(\rho_{s}\right)$.

Initial and calibrated values for the variables introduced above are listed in Table 5.3.

The sum of squares of the errors used to optimize the calibratable parameters was computed using the temperature of the exhaust gas downstream of the diesel oxidation catalyst. This signal contains information about the thermal inertia of the DOC in the form of time delay with respect to the exhaust temperature upstream of the DOC and 
Table 5.3: Thermal parameters used in this study.

\begin{tabular}{lccc}
\hline \hline Parameter & Initial Value & Calibrated Value & Units \\
\hline$A_{N}$ & 0.571 & 1.088 & - \\
$B_{N}$ & $2 / 3$ & 0.64 & - \\
$\lambda_{s}$ & 1 & 17.5 & $\mathrm{~W} / \mathrm{mK}$ \\
$\rho_{s}$ & 1800 & 2700 & $\mathrm{~kg} / \mathrm{m}^{3}$ \\
\hline \hline
\end{tabular}

therefore it appeared suitable to tune parameters that are related to heat transfer. As an example, a comparison between measured and simulated outlet temperature before the calibration process is shown in Figure 5.6; it is worth noting the considerable discrepancy between the two sets of data.

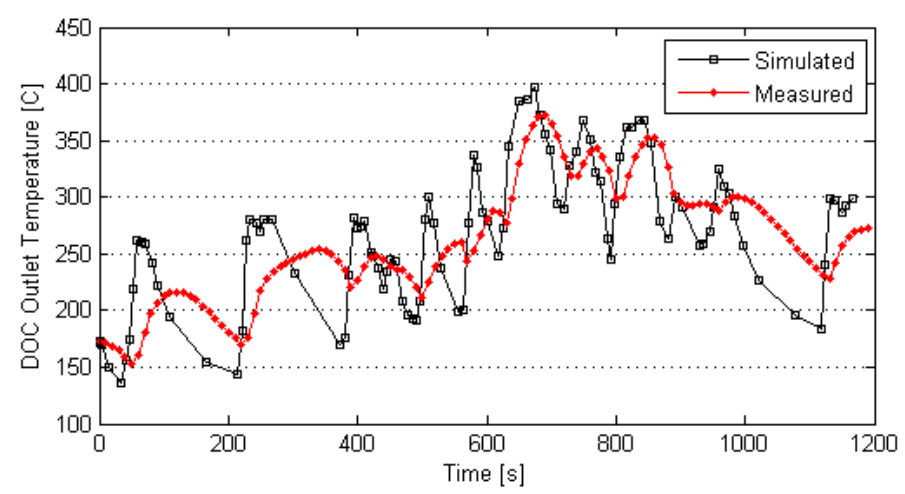

Figure 5.6: Simulated vs. measured exhaust temperature before the calibration process.

As long as gaseous species concentrations are concerned, for each chemical reaction included in the DOC model the optimization process involved the following properties:

1. Activation Energy $\left(E_{i}\right)$

2. Frequency Factor $\left(A_{i}\right)$

3. Constants $A_{S}$ and $B_{S}$ appearing in the generic Sherwood number correlation adopted in this work (equation 2.34).

The objective functions were computed using the experimental and simulated concentrations downstream of the diesel oxidation catalyst. Due to the effect of the cross 
inhibition factors the optimization process needed to be performed several times in order to obtain simultaneous minimization of the objective functions associated with $C O$, $H C$ and NO. Further, specific Sherwood number correlations were used for each species as previous studies showed that Sherwood number correlations may be chemical species dependent [85].

The experimental data used for the tuning of the DOC model included both steadystate (R10 and R100) and transient (FTP) data in order to improve the robustness of the calibration. The resulting set of parameters used to replicate the experimental data presented in this work are listed in Table 5.4 while comparisons between experimental and simulated results are discussed in the next chapter.

Table 5.4: Species conservation parameters used in this study.

\begin{tabular}{lccc}
\hline \hline Parameter & Initial Value & Calibrated Value & Units \\
\hline$E_{C O}$ & $1.1510^{5}$ & $1.1610^{5}$ & $\mathrm{~J} / \mathrm{mol}$ \\
$A_{C O}$ & $1.8210^{19}$ & $1.7810^{19}$ & $\mathrm{~m}^{3} \mathrm{~K} / \mathrm{mol} \mathrm{s}$ \\
$A_{S, C O}$ & 0.128 & 0.107 & - \\
$B_{S, C O}$ & 0.62 & 0.67 & - \\
$E_{H C}$ & $910^{4}$ & $9.42410^{4}$ & $\mathrm{~J} / \mathrm{mol}$ \\
$A_{H C}$ & $4.710^{20}$ & $4.60610^{20}$ & $\mathrm{~m}^{3} \mathrm{~K} / \mathrm{mol} \mathrm{s}$ \\
$A_{S, H C}$ & 0.128 & 0.0129 & - \\
$B_{S, H C}$ & 0.62 & 0.984 & - \\
$E_{N O}$ & $8.610^{7}$ & $7.68810^{7}$ & $\mathrm{~J} / \mathrm{mol}$ \\
$A_{N O}$ & $2.0810^{12}$ & $6.2410^{11}$ & $\mathrm{~m}^{3} \mathrm{~K} / \mathrm{mol} \mathrm{s}$ \\
$A_{S, N O}$ & 0.128 & 0.0117 & - \\
$B_{S, N O}$ & 0.62 & 0.706 & - \\
\hline \hline
\end{tabular}




\section{Chapter 6}

\section{Results and Discussion}

This chapter discusses the comparison between measured data and simulated values, in an effort to provide validation for the DOC-DPF model. Results have been organized per engine cycle (R10, FTP, R100). As long as the DOC model is concerned, results from both the base and simple models are presented and compared to experimental values.

\subsection{DPF Loading Cycle}

\subsubsection{Engine Performance and Emissions}

Results in terms of engine performance and average brake specific emissions for the R10 engine cycle are presented in Table 6.1.

Table 6.1: Average brake specific emissions for the R10 engine cycle.

\begin{tabular}{lcc}
\hline \hline Parameter & Value & Units \\
\hline EngineSpeed & 1800 & $\mathrm{rpm}$ \\
EngineTorque & 105 & $\mathrm{ft}-\mathrm{lb}$ \\
FuelRate & 1.621 & $\mathrm{~g} / \mathrm{s}$ \\
$\mathrm{HC}$ & 0.0234 & $\mathrm{~g} / \mathrm{bhp}-\mathrm{hr}$ \\
$\mathrm{NO}_{x}$ & 2.399 & $\mathrm{~g} / \mathrm{bhp}-\mathrm{hr}$ \\
$\mathrm{CO}$ & 0.0349 & $\mathrm{~g} / \mathrm{bhp}-\mathrm{hr}$ \\
$\mathrm{CO}_{2}$ & 982.6 & $\mathrm{~g} / \mathrm{bhp}-\mathrm{hr}$ \\
\hline \hline
\end{tabular}


Further, exhaust emissions were sampled raw and results in terms of concentrations are provided in Table 6.2 at three different locations over the aftertreatment system.

Table 6.2: Average raw emissions measured over the R10 cycle.

\begin{tabular}{lccc}
\hline \hline Pollutant & Pre-DOC & Pre-DPF & Post-DPF \\
\hline$H C[p p m]$ & 96.8 & 19.3 & 2.7 \\
$C O[p p m]$ & 162 & 1.9 & 2.2 \\
$N O_{x}[p p m]$ & 170 & 171 & 168.7 \\
$N O[p p m]$ & 136 & 56 & 53 \\
$N O_{2}[\mathrm{ppm}]$ & 34 & 114 & 112 \\
$P M\left[\mathrm{mg} / \mathrm{m}^{3}\right]$ & 1.8 & - & - \\
\hline \hline
\end{tabular}

It is worth noting that $N O_{x}$ emissions are constant over the three locations as the CCRT system is not supposed to reduce oxides of nitrogen; the slight oscillations that can be observed in Table 6.2 are attributed to experimental errors. On the other hand, the $\mathrm{NO} / \mathrm{NO}_{x}$ ratio is altered significantly going from 0.8 at the engine-out to 0.33 downstream of the diesel oxidation catalyst.

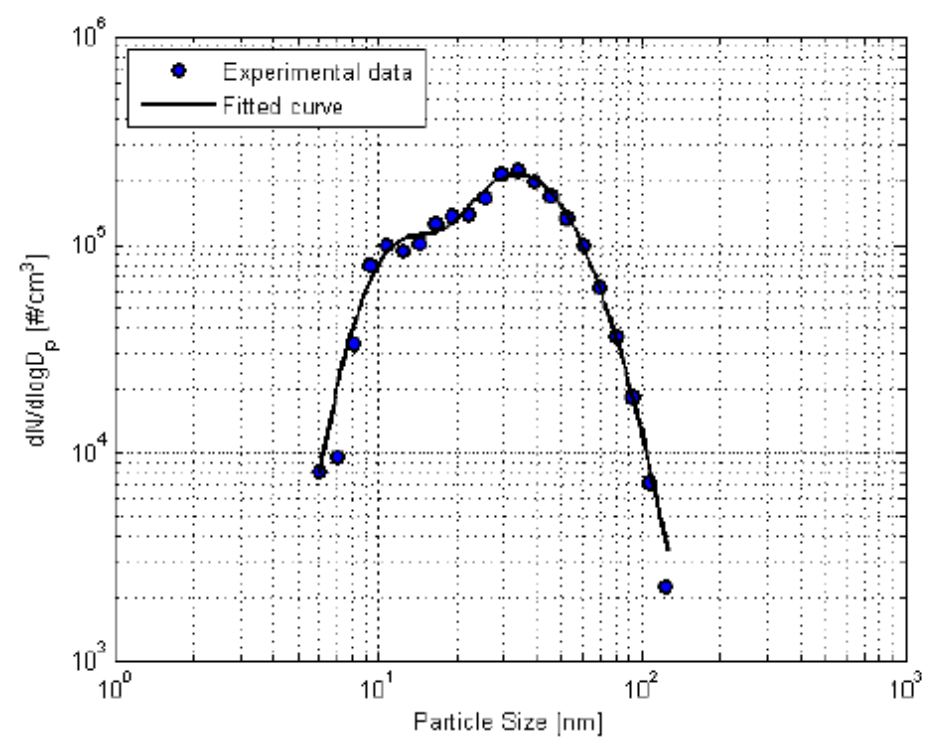

Figure 6.1: Time averaged particle size distribution entering the C-CRT over the R10 cycle. 
Concurrently to gaseous emissions, engine-out PM concentration and particle size distribution have been acquired to complete the set of data required as input to the DPF model. Access to size resolved PM measurements allows for correct computation of filtration efficiencies used for the replication of the deep bed filtration mechanism. Although cake filtration has been showed to be the primary collection mechanism for the C-CRT used in this study, correct estimation of wall filtration efficiency is of primary importance during the virtual conditioning process. Particle size average spectrum collected upstream the diesel oxidation catalyst is provided in Figure 6.1 in terms of the 32 EEPS channels and fitted lognormal distribution.

\subsubsection{DOC Model}

The diesel oxidation catalyst model provides estimations for the outlet concentrations of $N O, C O$, and $H C$ as well as outlet exhaust temperature. Since the calibration process was performed on the base model, at first simulated results from this model were compared to measured data. Once the accuracy of the base model was verified, the validation was moved forward to the simple model.

As discussed in the previuos chapters, differences between the two DOC models are confined to the methodology used to solve the equation of species conservation. Therefore, results from the thermal module in terms of exhaust outlet temperature and substrate temperature are common to the two models and can be discussed independently.

Comparison between simulated and measured values for the outlet exhaust temperature in terms of continuous traces is provided in Figure 6.2. Accuracy of the thermal module in replicating exhaust temperature downstream the DOC appears to be satisfactory, with an average simulated value of $222.4^{\circ} \mathrm{C}$ compared to a measured value of $224.7^{\circ} \mathrm{C}$. Further, simulated values appear to catch also the initial transient behavior depicted in the insertion of Figure 6.2. 


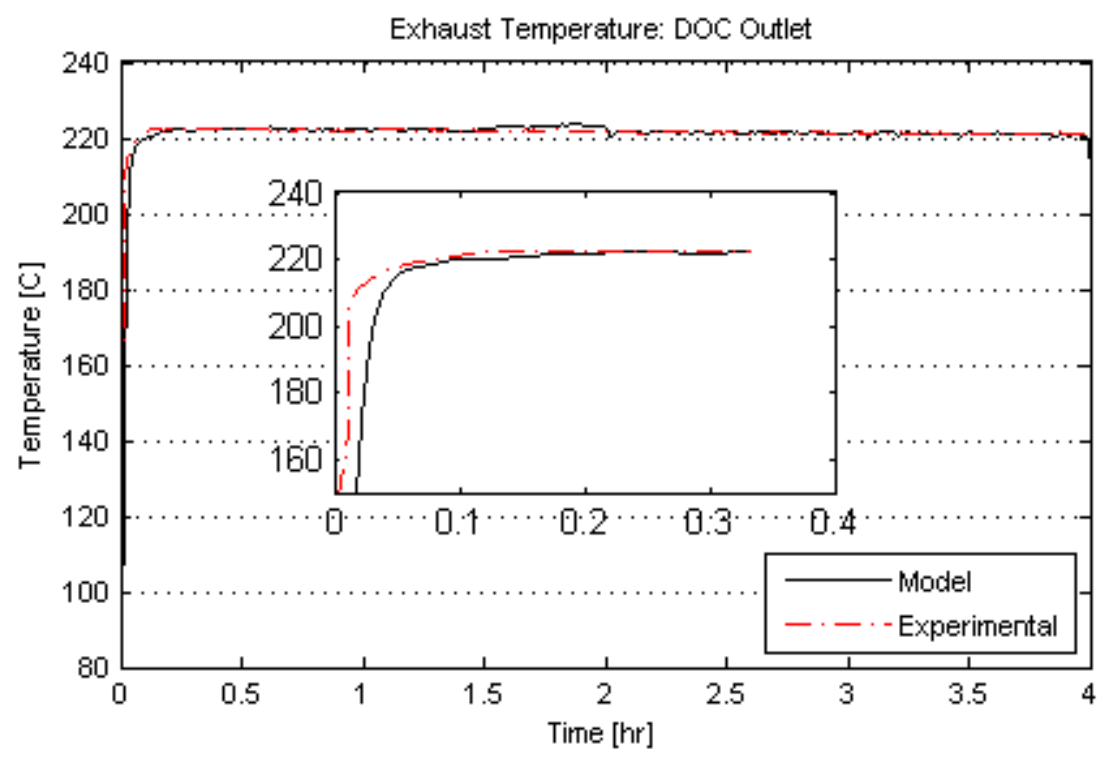

Figure 6.2: Simulated and measured outlet temperature during the R10 cycle.

Additional results generated by the thermal module are in terms of substrate temperature. A critical aspect that has major impact on the estimation of the conversion efficiencies is in fact the warming-up of the substrate. Evolution of the substrate temperature at different axial locations as a function of time is provided in Figure 6.3.

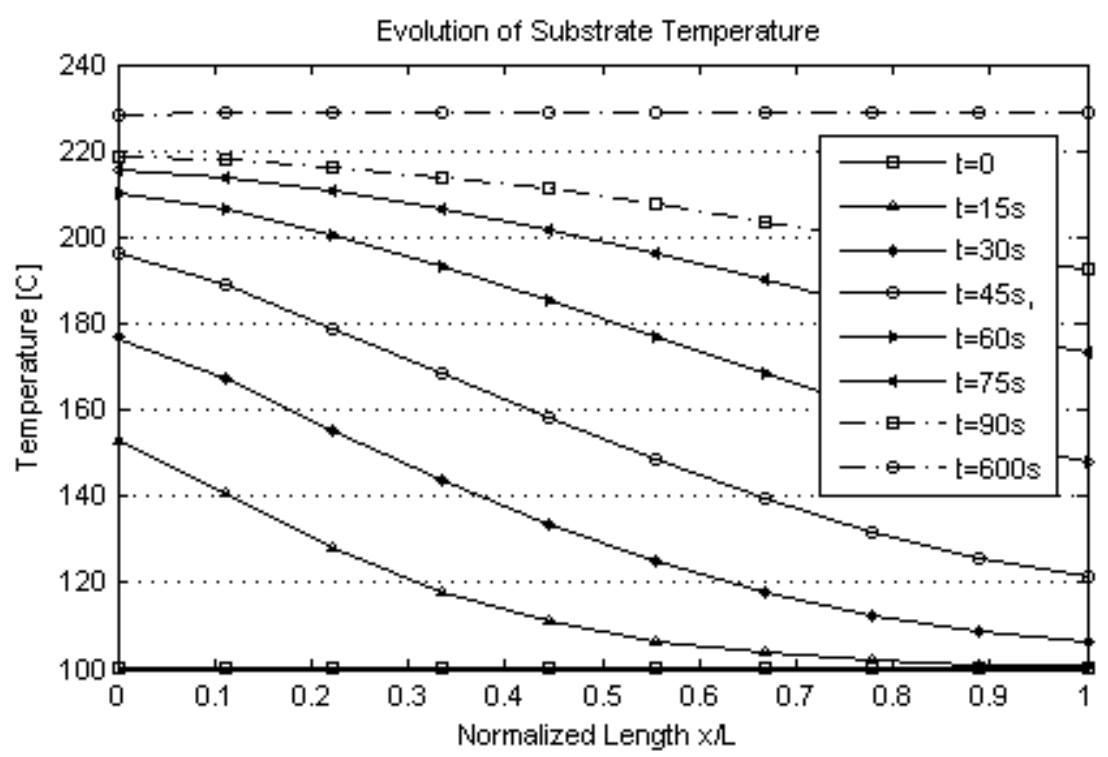

Figure 6.3: Evolution of substrate temperature during DOC warm-up.

It is worth noting how the modeled thermal inertia and heat conduction affect the temperature of the wall, with the front part of the DOC reaching a steady-state condition in a shorter time than the back. 
The DOC+DPF model configuration including the complete solution of the species conservation equation was capable of replicating 4 hours of experimental data in a time $t_{\text {base }, R 10}=12.8 s$ when running on a machine equipped with a Pentium $41.83 \mathrm{GHz}$. On the same machine, the simplified model was abale to replicate the same experimental data in a time $t_{\text {simple }, R 10}=5.2 \mathrm{~s}$. Results in terms of average concentrations downstream the diesel oxidation catalyst are reported in Table 6.3 for one of the R10 cycles, specifically test \#2.

Table 6.3: Simulated and measured average raw concentrations downstream the DOC over the R10 cycle.

\begin{tabular}{lccc}
\hline \hline Pollutant & Base Model $[\mathrm{ppm}]$ & Simple Model $[\mathrm{ppm}]$ & Measured [ppm] \\
\hline$H C$ & 15.65 & 14.78 & 18.2 \\
$C O$ & 0.43 & 0.52 & 0.61 \\
NO & 60.16 & 57.3 & 58.85 \\
\hline \hline
\end{tabular}

Results from Table 6.3 indicate that raw emissions concentrations are estimated within a $14 \%$ error in case of $C O$ and $H C$, while the error reduces to $2.3 \%$ in case of $N O$. The discrepancies that are observed in case of carbon monoxide and total hydrocarbons can be attributed to: i) the simplified speciation of the hydrocarbons which in this study were assumed to be represented by $\mathrm{C}_{3} H_{6}$ and ii) by the extremely low levels of $C O$ measured downstream the diesel oxidation catalyst. However, it is noteworthy to point out that discrepancies of $14 \%$ on emissions concentrations of the order of 1-10 ppm can be considered small and have minimal effect on the computation of emissions on a mass basis. Continuous traces for $N O, H C$, and $C O$ concentrations downstream the DOC are presented in Figure 6.4 which shows good agreement between measured and experimental time resolved data.

Concentration distribution over the channel length for the three species of interest is depicted in Figure 6.5; it can be noted how the final model calibration is such to have very efficient conversion of carbon monoxide in just half of the available length. This 

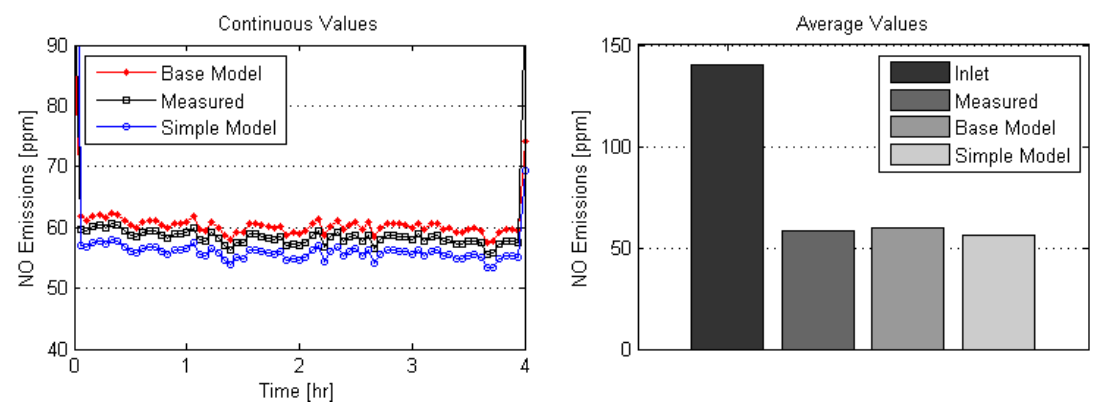

(a) Nitric Oxide
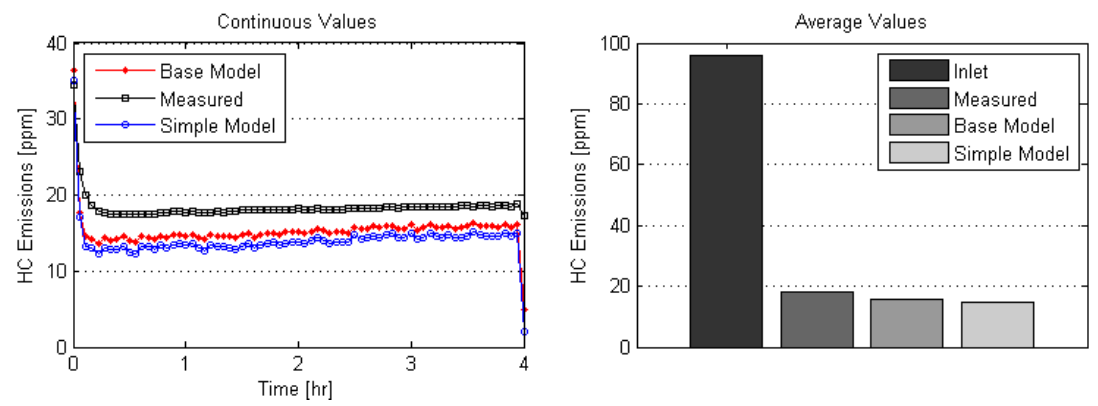

(b) Hydrocarbons
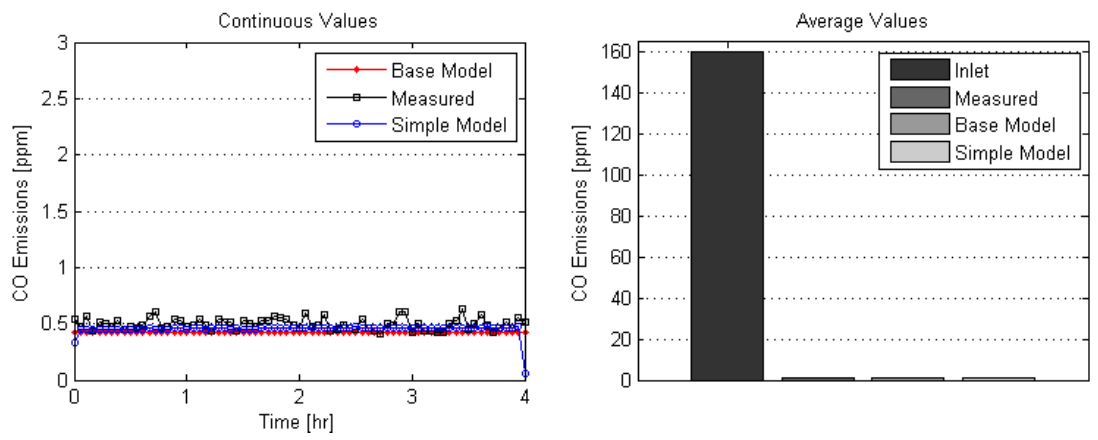

(c) Carbon Monoxide

Figure 6.4: Continuous emissions traces during the R10 cycle.

fact is in agreement with other studies available in the literature and it is explained by the values of frequency factor and activation energy used to compute $C O$ oxidation rate. On the other hand, maximum conversion efficiencies of hydrocarbons and nitric oxide are attained at the outlet of the diesel oxidation catalyst due to the lower reaction rates and, in case of $N O$, the onset of thermodynamic equilibrium. Data displayed in Figure 6.5 were obtained using the base model.

Further comparison between simulated and experimental is presented in terms of cumulative mass of pollutants leaving the diesel oxidation catalyst. For each species the cumulative mass was computed by integration of mass flow rate over time, as shown in 


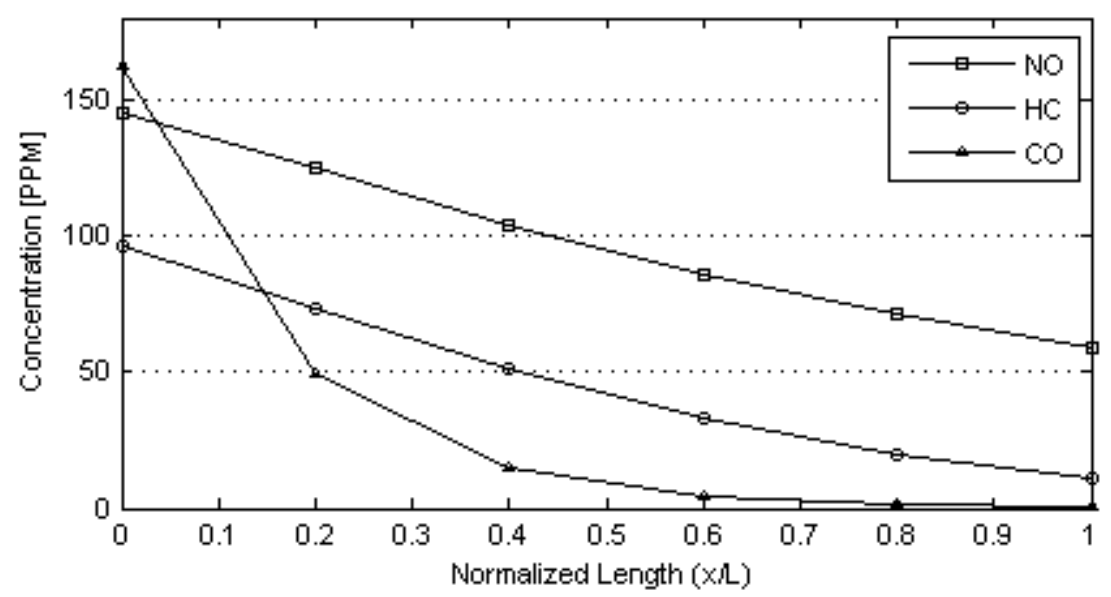

Figure 6.5: Evolution of inlet concentrations over the length of the DOC.

equation 6.1:

$$
m_{i, t_{\text {end }}}=\int_{0}^{t_{\text {end }}} \dot{m}_{i}(t) d t
$$

where $i=N O, C O, H C$. The mass flow rate of each species was computed using the molar concentration in the raw exhaust according to equation 6.2:

$$
\dot{m}_{i}(t)=y_{i}(t) \times M W_{i} \times \dot{V}_{\text {exhaust }}(t)
$$

In equation $6.2, \dot{V}_{\text {exhaust }}$ denotes the exhaust molar flow rate (units $\mathrm{mol} / \mathrm{s}$ ) while $M W_{i}$ is the molecular weight of component $i$. Analysis of the results depicted in Figure 6.6 reveals that:

- Adoption of the diesel oxidation catalyst led to a reduction of $58.72 \%$ in nitric oxide emissions. Further, after 4 hours of steady-state operation the discrepancies between experimental data and simulated values are limited to $+3.85 \mathrm{~g}(3.14 \%$ error $)$ and $-4.79 g$ (3.91\% error) for the base and simple model respectively. It is worth noting that although the same calibration was used for the two models, the model with the simplified chemistry tends to underestimate NO emissions when compared to the more complex model.

- Results in terms of hydrocarbons show a reduction of $83.84 \%$ in total HC mass 
emitted; comparison between simulated and measured data shows discrepancies of $-2.01 g(4.49 \%)$ and $-3.16 g(7.05 \%)$ for the complete and simplified model respectively. In case of hydrocarbons, both models appear to underestimate the measured values.

- Carbon monoxide experiences a reduction of 99.65\%. Relative discrepancies and errors are not as meaningful as in case of $\mathrm{NO}$ and $\mathrm{HC}$ due to the extremely low concentrations measured downstream the diesel oxidation catalyst (order of $1 \mathrm{ppm}$ ).
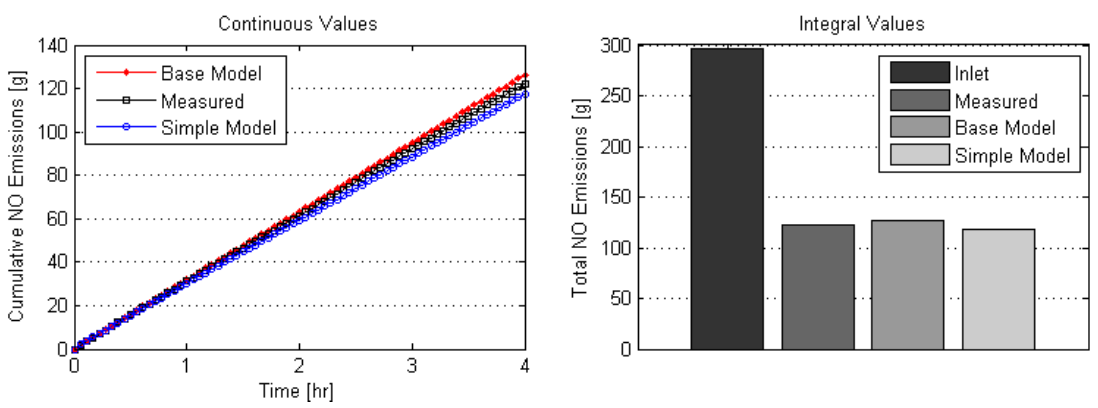

(a) Nitric Oxide
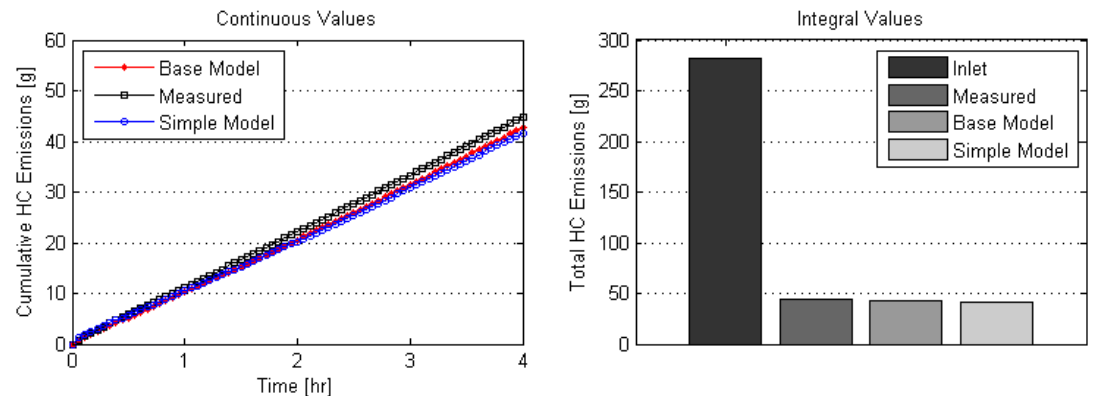

(b) Hydrocarbons
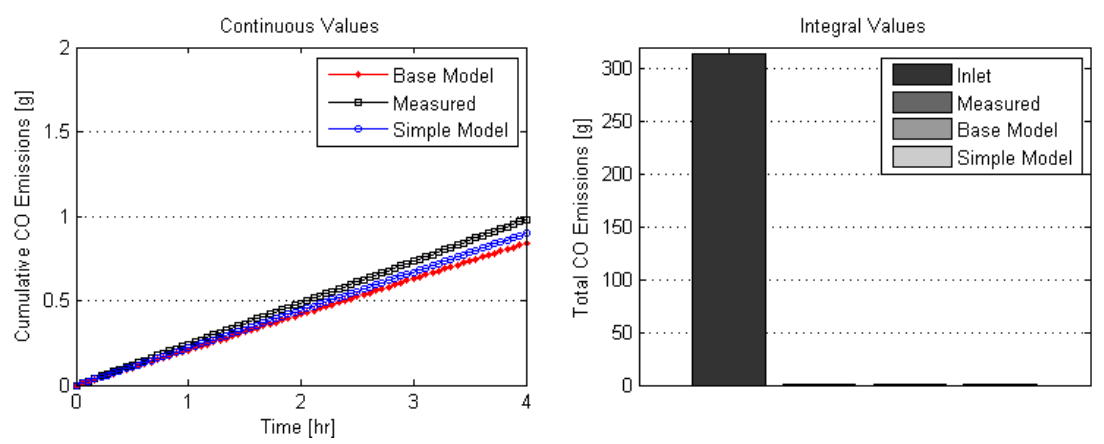

(c) Carbon Monoxide

Figure 6.6: Cumulative and integral mass emissions over the R10 cycle. 
In order to provide an overview of the performance of the two DOC models during the whole DPF loading process, results in terms of total mass emitted have been summarized and organized in Figure 6.7.
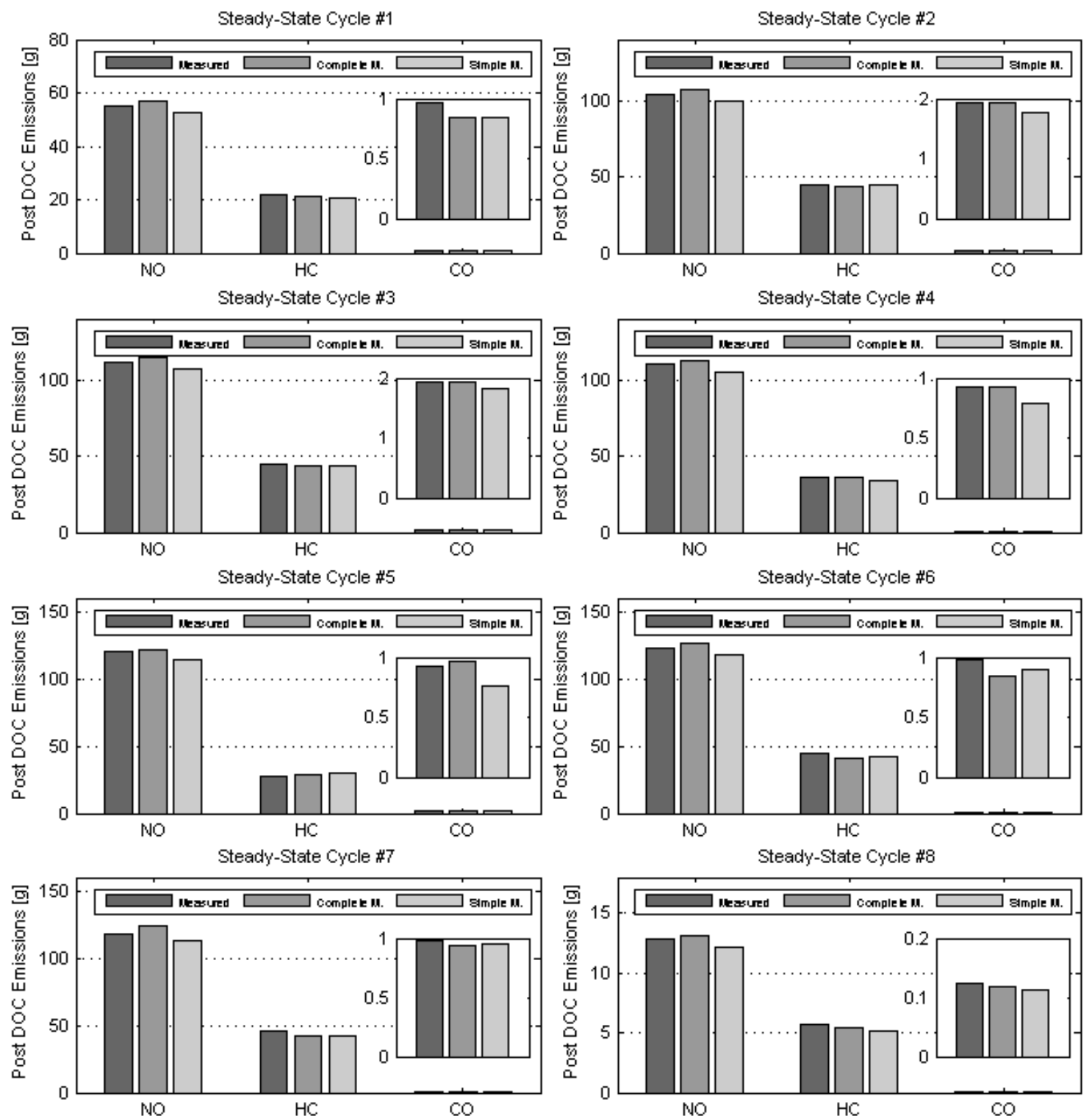

Figure 6.7: Overview of the performance of the two models during DPF loading.

\subsubsection{DPF Model}

The comparison between experimentally gathered data and calculated results has been performed via two indicators: differential pressure across the diesel particulate filter and weighed mass as function of time. At first, a valid proof for the hyphotesis of conditioned 
filter is presented in Figure 6.8 which shows an experimental pressure drop obtained by fitting the pressure curves associated with the individual loading cycles (R10s).

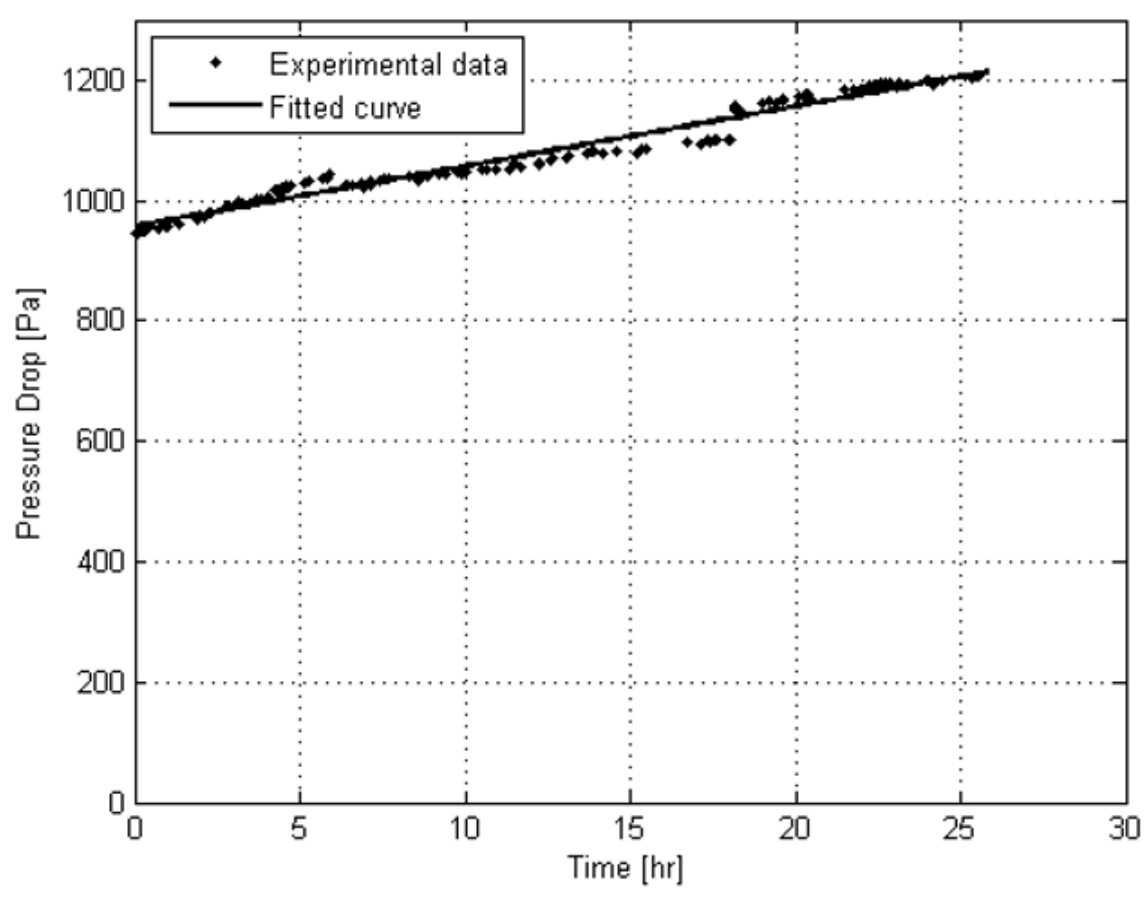

Figure 6.8: Overall filter pressure during the loading cycle.

Typical pressure drop curves of clean DPFs show a non-linear behavior during the initial phase of the loading process; as explained in the previous chapter the non-linear profile results from the deposition of soot particles within the substrate wall. Even small amounts of mass can lead to sudden increase of pressure drop across the filter wall. However, this non-linear behavior is not present in Figure 6.8 which, on the contrary, shows a linear increse of pressure drop with time. The linearity in pressure drop curve is a characteristic of conditioned/aged filters collecting PM mass by accumulation over the wall surface, hence it can be retained a valid proof for the assumption used in this work.

The loading process is summarized in Figure 6.9 and Figure 6.10 which show experimental versus simulated results in terms of filter pressure drop over the individual R10 cycles. 

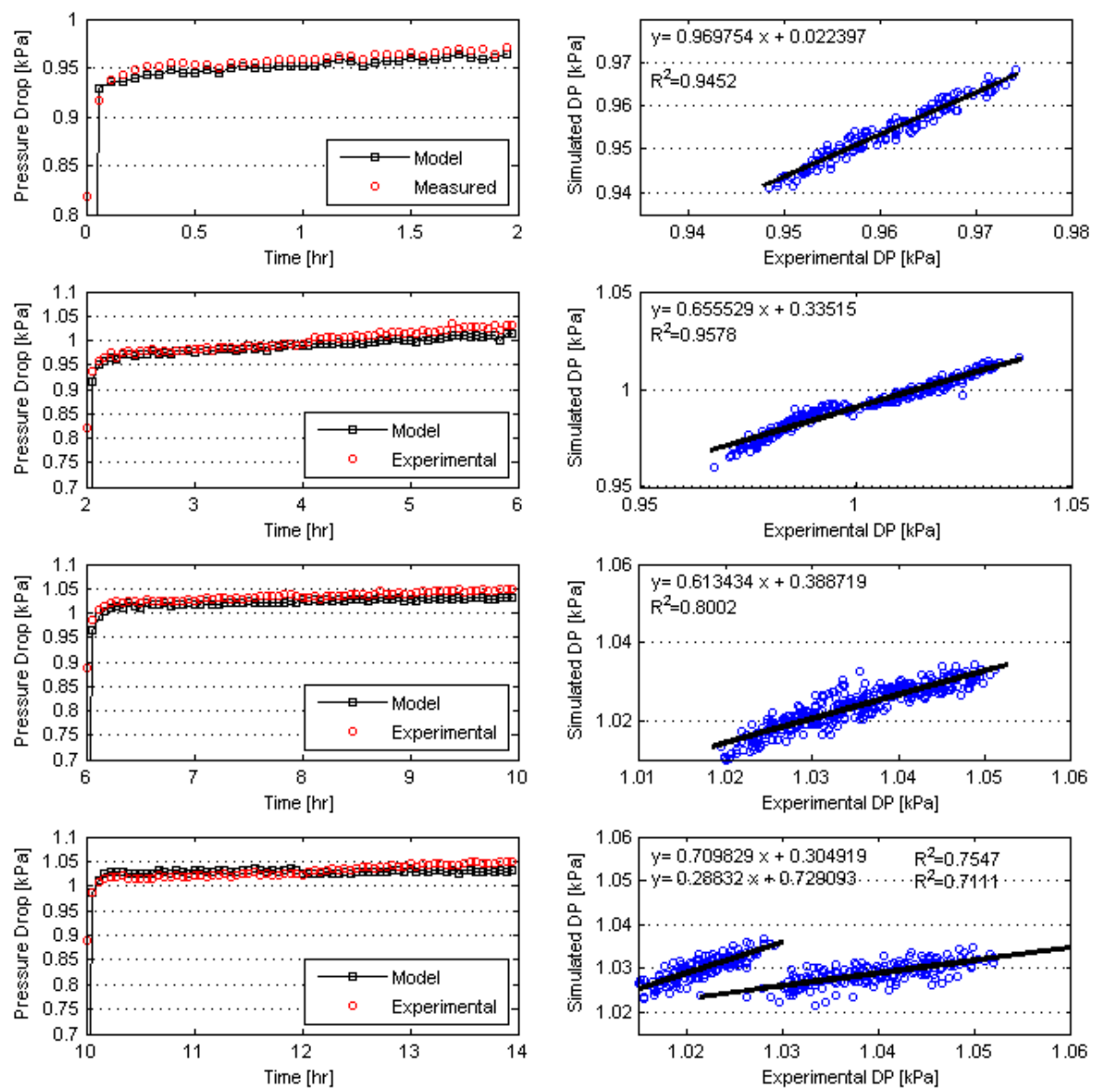

Figure 6.9: Measured versus simulated filter pressure drop during the first phase of the loading process

Analysis of Figure 6.9 and Figure 6.10 reveals the presence of a non-linear behavior during the first part (15-20 min) of each loading cycle; although this can appear contradictory with the assumption of conditioned filter, a different reason for the non-linear trend can be provided if the temperature of the DPF is taken into account. In fact, the first portion of each loading cycle represents a small transient cycle in which the DPF goes from an initial temperature $T_{0}$ to the equilibrium temperature $T_{\text {end }}$ as depicted in Figure 6.11.

It is worth noting that the values shown in Figure 6.11 are for simulated DPF substrate temperature and measured post-DPF exhaust gas temperature; this is due to the 1-D assumption used to build the DPF model and implies that, at steady-state conditions, 

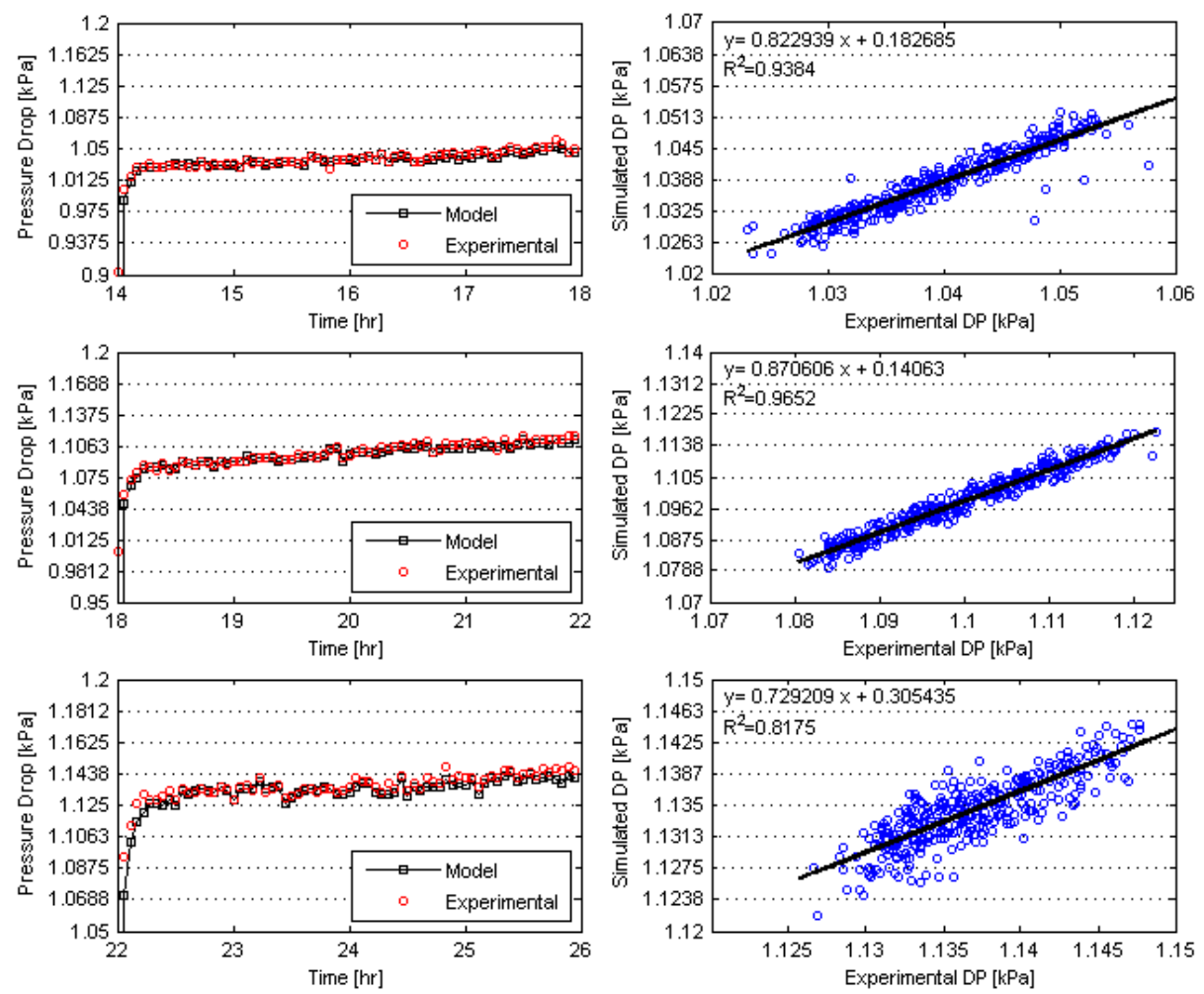

Figure 6.10: Measured versus simulated filter pressure drop during the last phase of the loading process

substrate and exhaust gas leaving the filter are close to thermal equilibrium.

Results in terms of filter pressure drop testify the model capabilities in tracking this parameter over time for consecutive engine cycles giving results in agreement with both thermal transient operation and steady-state conditions. According to the pressure drop model, the thickness of the soot layer is directly proportional to the filter pressure drop and, as it can be noted in Figure 6.9 and Figure 6.10, the gradient of the pressure curves during the first part of the loading process is higher than that of the last portion. This indicates the presence of passive $\mathrm{NO}_{2}$ regeneration taking place at low exhaust temperature. The reaction rate for the regeneration process appears to increase with time as more soot is accumulated over the wall surface. This process is represented in Figure 6.12 which shows the PM mass balance within the DPF during the whole loading process. 

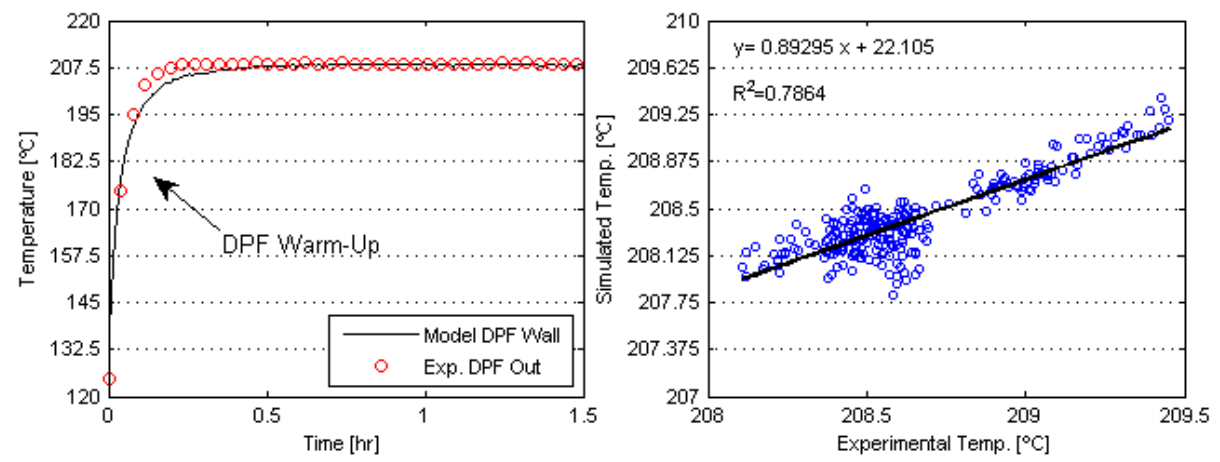

Figure 6.11: Measured post-DOC exhaust temperature compared to simulated DPF temperature.

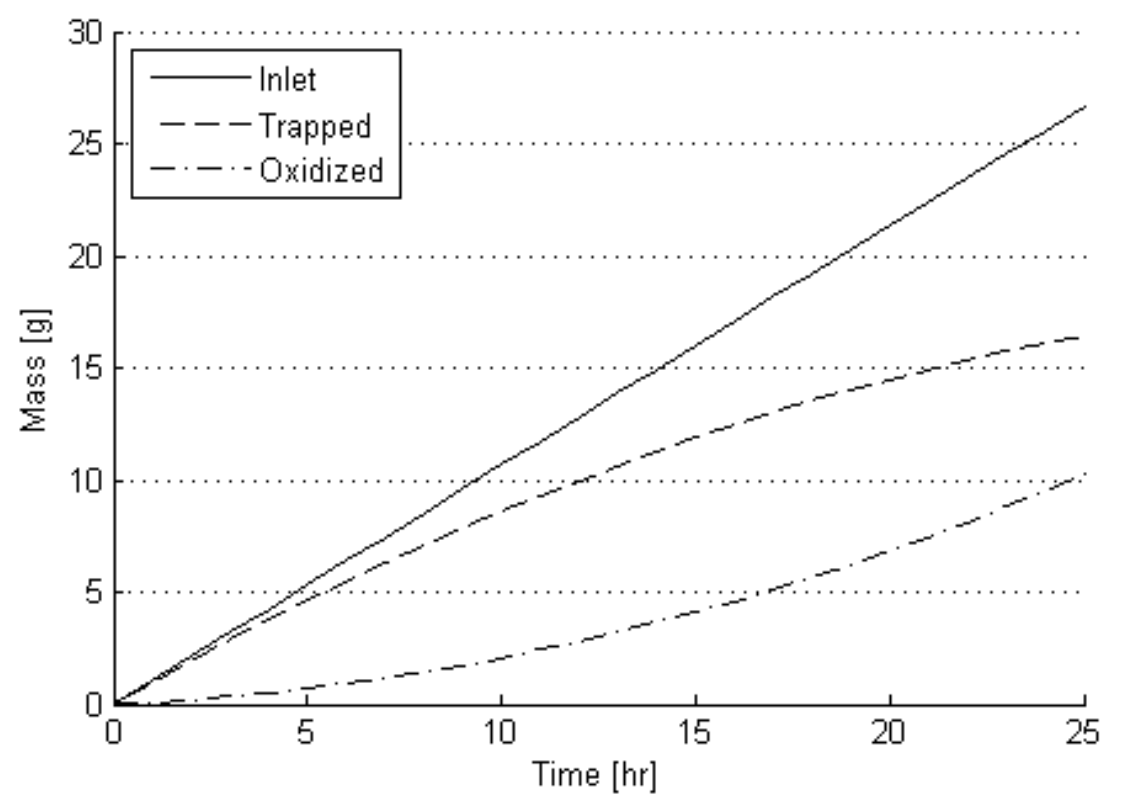

Figure 6.12: Simulated PM mass balance within the DPF.

Inspection of Figure 6.12 reinforces what was discussed earlier, with the oxidation rate of trapped PM increasing as more particles are collected in the filter. As soot accumulation increases over time, low temperature oxidation becomes more efficient leading the C-CRT to end the loading process with a net soot content of approximately $0.8 \mathrm{~g} / \mathrm{l}$. This behavior further depends on the performance of the DOC which operates already very efficiently as discussed in the previous section. Results presented in Figure 6.13 show the distribution of the total trapped and oxidized mass by location. The figure points out to the fact the total PM oxidized in the PM cake layer is much higher than the PM oxidized within the substrate wall. This is due to the PM cake acting like a filter and retaining the 
majority of PM in the cake layer itself. This ultimately enhance the oxidation process in the cake layer since more PM is available for oxidation at this location.

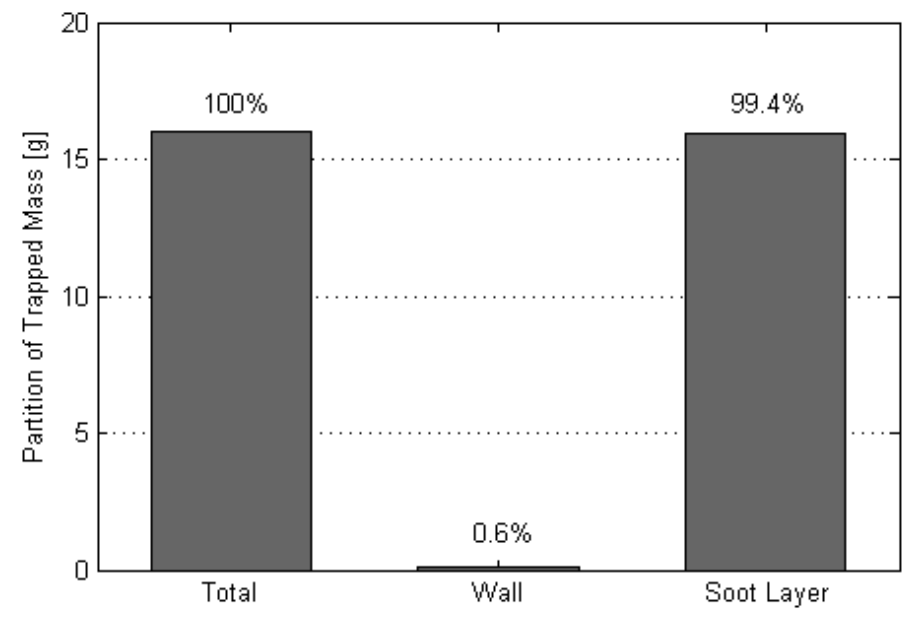

(a) PM Trapped Mass

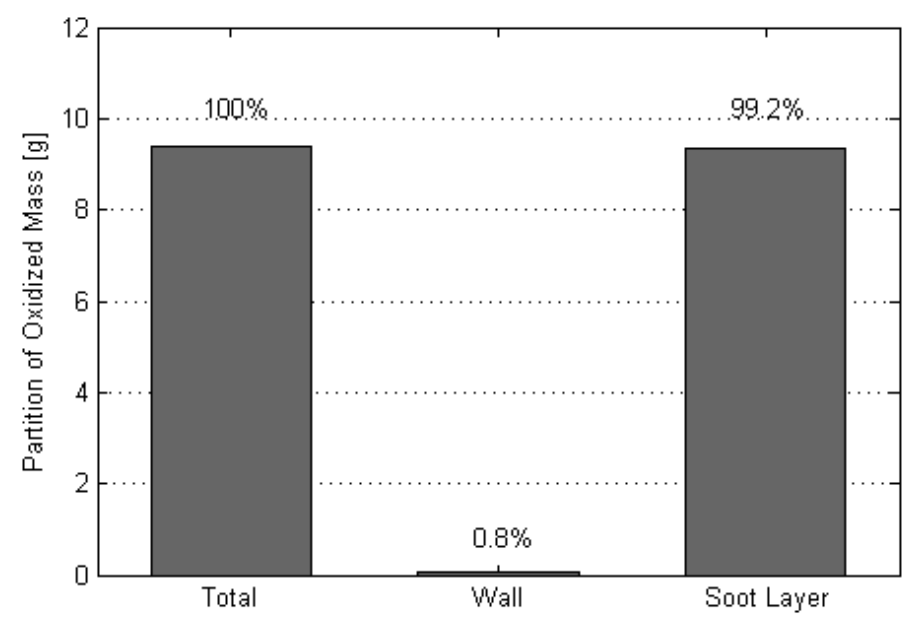

(b) PM Oxidized Mass

Figure 6.13: Distribution of computed PM mass within the DPF.

It is worth noting that although the filter is considered to be conditioned there is still a small portion of the inlet PM mass which is trapped in the substrate wall. This is ultimately made possible by the original mass balance that was implemented in the DPF model and that uses a combination of the wall percolation factor and cake layer filtration efficiency to manage the deposition of PM mass (Figure 3.5). This is believed to be a critical aspect in DPF modeling as minimal amount of PM loading in the wall can result in significant increase in filter pressure drop due to changes in wall properties. 
An example of the evolution of wall properties during the loading process is depicted in Figure 6.14 which shows both wall permeability and virtual soot later per unit thickness at different points in time as function of the normalized wall thickness.

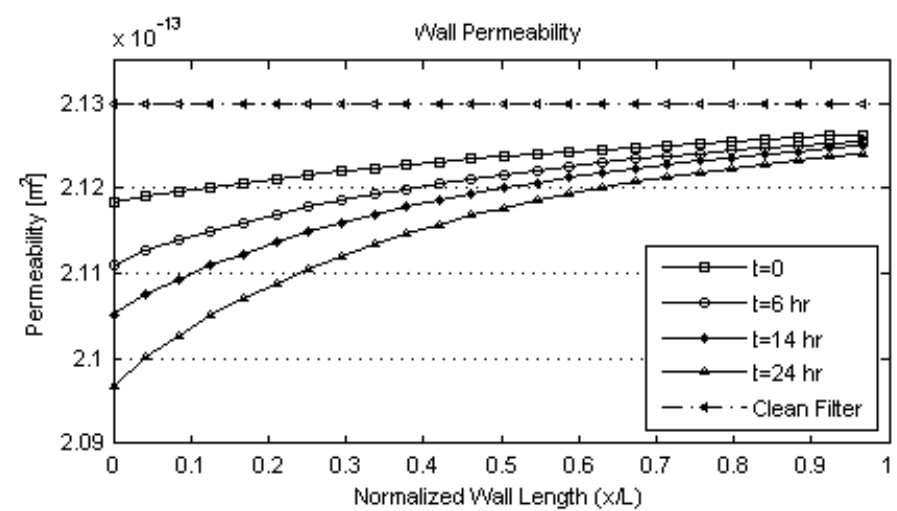

(a) Wall Permeability

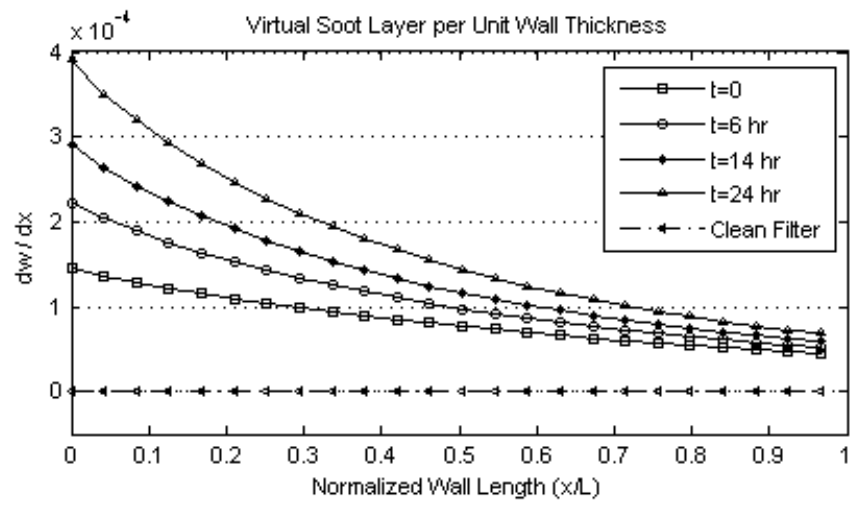

(b) Soot Layer per Unit Wall Thickness

Figure 6.14: Distribution of computed PM mass within the DPF.

It is worth noting that properties at time $t=0$ differ from those of a clean filter as result of the virtual conditioning process. Although final values are still significantly low, a variation with respect to time is observable for both the parameters. Moreover, the frontal section of the wall appears to experience higher soot loading than the rear section indicating that the theoretical wall capacity in terms of particle collection is never fully exlpoited. Further, the importance of correctly capture the evolution of wall properties is clear when Figure 6.15 is analyzed. Partition of total pressure drop into different pressure components shows how the pressure drop across the wall represents the main contribution even in case of conditioned filter. Soot loading and oxidation in the substrate wall are therefore key parameters that need to be simulated accurately. 


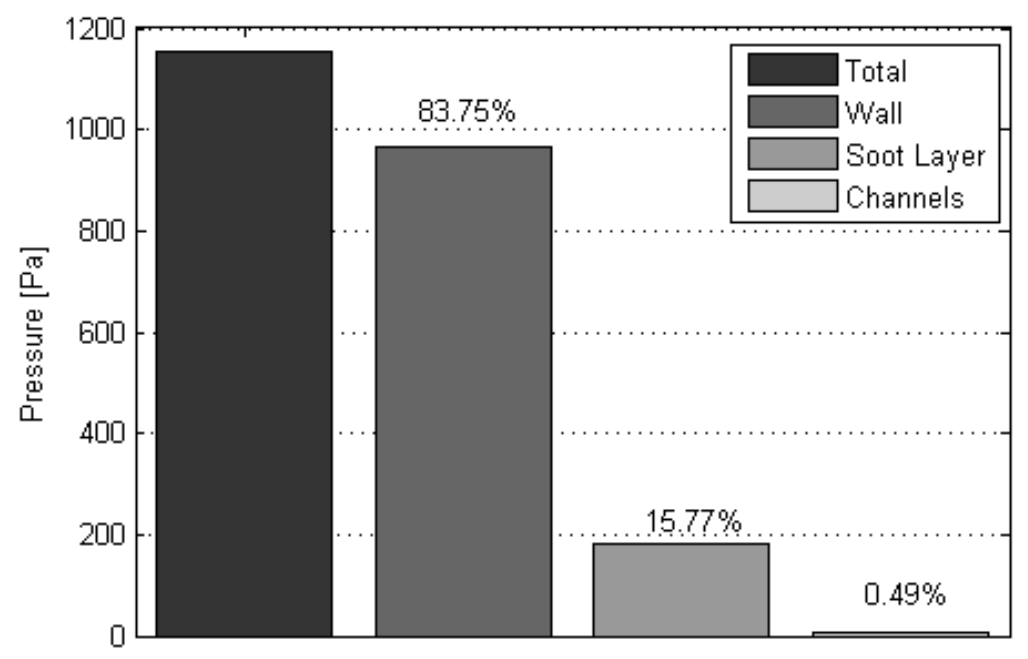

Figure 6.15: Partition of overall pressure drop into sub-components.

Model prediction for the total PM mass trapped within the filter as function of time is illustrated in Figure 6.16. The figure shows satisfactory agreement between experimental and simulated results. Deviations between the two results are within the resolution of the scale used to perform the weighing measurements and appear to increase slightly towards the end of the loading process. It is worth noting that the maximum discrepancy of $3.1 \mathrm{~g}$ is smaller than the accuracy of the scale used in this work $( \pm 5 \mathrm{~g})$. In light of a tuning process involving data generated over different engine cycles, the error tolerance observed during the loading process has been considered satisfactory.

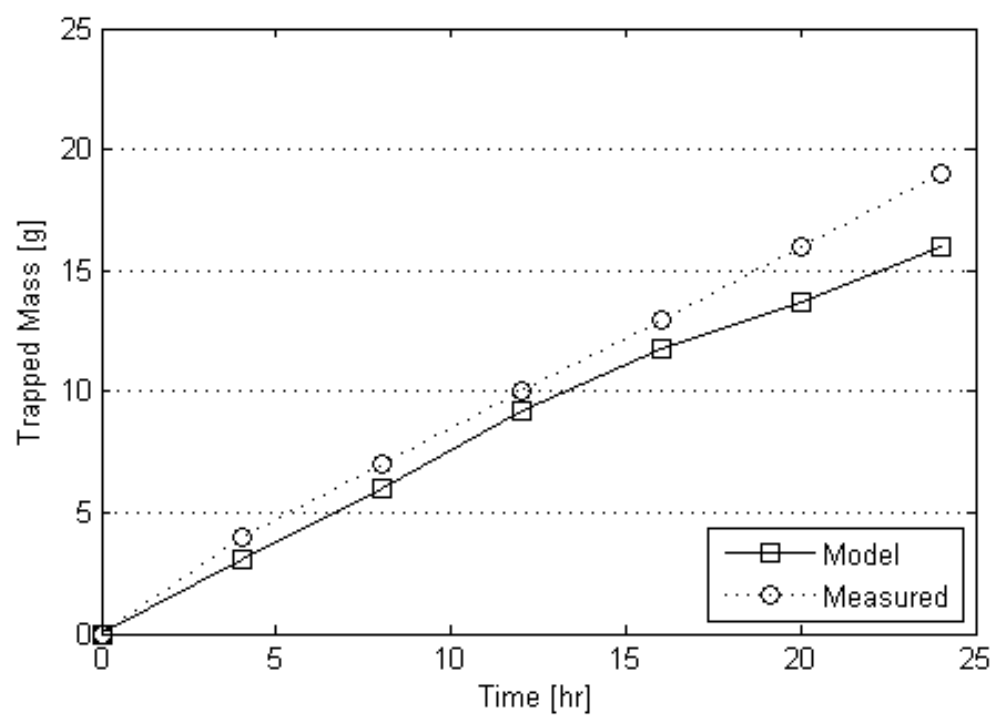

Figure 6.16: Filter soot load: experimental versus simulated results. 


\subsection{Transient Cycle: Federal Testing Procedure (FTP)}

\subsubsection{Engine Performance and Emissions}

The transient portion of the test matrix included two consecutive FTP engine cycles after the $25 \mathrm{hr}$ of filter loading. FTP cycle was selected as transient cycle mainly because the performance of any OBD monitor has to be tested over the FTP cycle before it can be implemented in the engine ECU. Therefore, it is imperative for aftertreatment models to be able to reproduce FTP experimental results if OBD monitors have to be developed following a model-based approach. Further, this engine cycle can be divided in two main portions depending on the average exhaust temperature. This fact gives the opportunity to test the model calibration on a wide range of operating points as discussed later in the chapter.

Inputs to the DOC and DPF model are provided in Figure 6.17 whilst emissions results in terms of brake specific values are presented in Table 6.4.

Table 6.4: Engine emissions over the FTP cycles: brake specific results.

\begin{tabular}{lccc}
\hline \hline Gaseous Specie & FTP 1 & FTP 2 & Units \\
\hline $\mathrm{HC}$ & 0.001343 & 0.001413 & $\mathrm{~g} / \mathrm{bhp}-\mathrm{hr}$ \\
$\mathrm{NO} \mathrm{O}_{x}$ & 2.136 & 2.141 & $\mathrm{~g} / \mathrm{bhp}-\mathrm{hr}$ \\
$\mathrm{CO}$ & 0.0431 & 0.0452 & $\mathrm{~g} / \mathrm{bhp}-\mathrm{hr}$ \\
$\mathrm{CO}_{2}$ & 528.56 & 527.12 & $\mathrm{~g} / \mathrm{bhp}-\mathrm{hr}$ \\
$\mathrm{PM}$ & 0.0128 & 0.0134 & $\mathrm{~g} / \mathrm{bhp}-\mathrm{hr}$ \\
\hline \hline
\end{tabular}

It is worth noting that the adoption of a transient cycle in the calibration/validation process is of primary importance to explore the capabilities of the in-house models to capture the physical behavior of the modeled components. In fact, steady-state engine modes such those used in standard model calibrations are not fully representative of realworld applications which, on the contrary, include transient operation. Further, adoption 
of raw signals such those depicted in Figure 6.17 allow for accurate estimation of the quantities needed as input by the computational model, overcoming the limitations of methods such those based on the extrapolation of emissions data from static maps and that have been shown to lead to unacceptable numerical deviations [144].
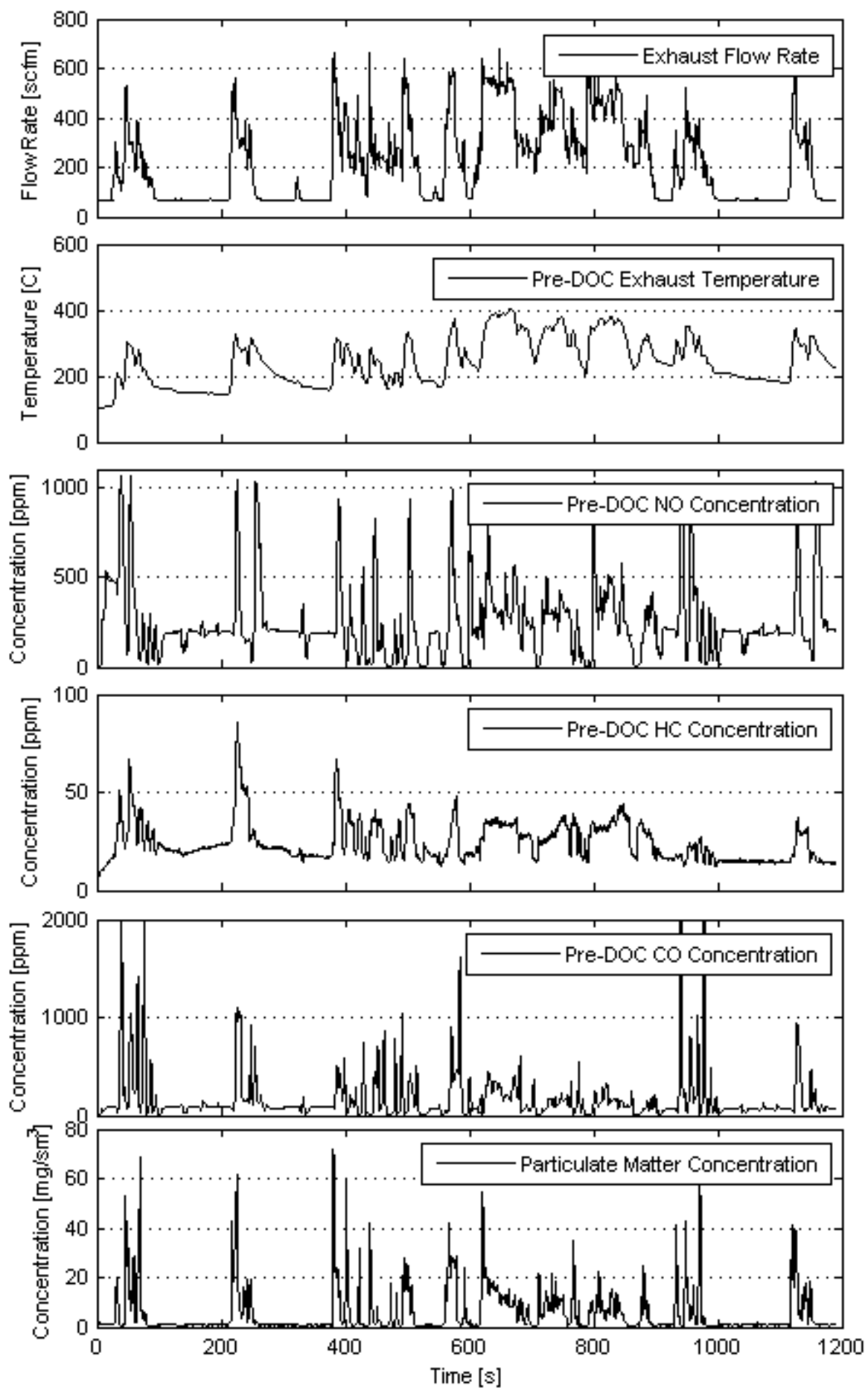

Figure 6.17: Input to the DOC and DPF model during the FTP cycle. 


\subsubsection{DOC Model}

Similarly to what presented in the previuous section, results generated by the DOC model are in terms of outlet concentrations for the species of interest. However, given the transient nature of the engine cycle, accurate prediction of the temperature of both susbstrate wall and engine exhaust exiting the DOC are of primarily importance as well.

Correct estimation of wall temperature allows for correct computation of the reactions rates used in the species conservation equation. On the other hand, the temperature of the exhaust leaving the DOC is critical as it represents an input for the DPF model and affects directly the oxidation process within the DPF.

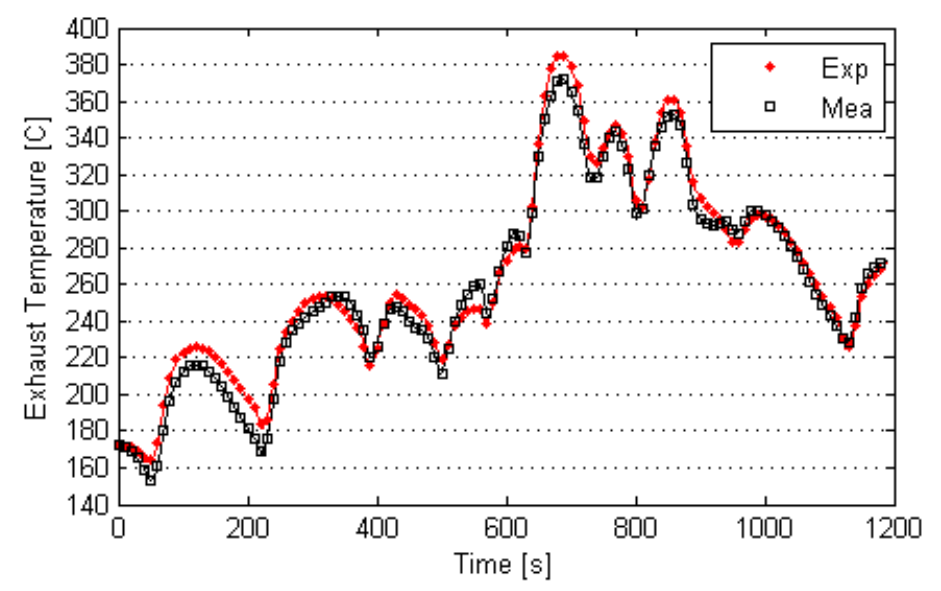

(a) FTP Cycle 1

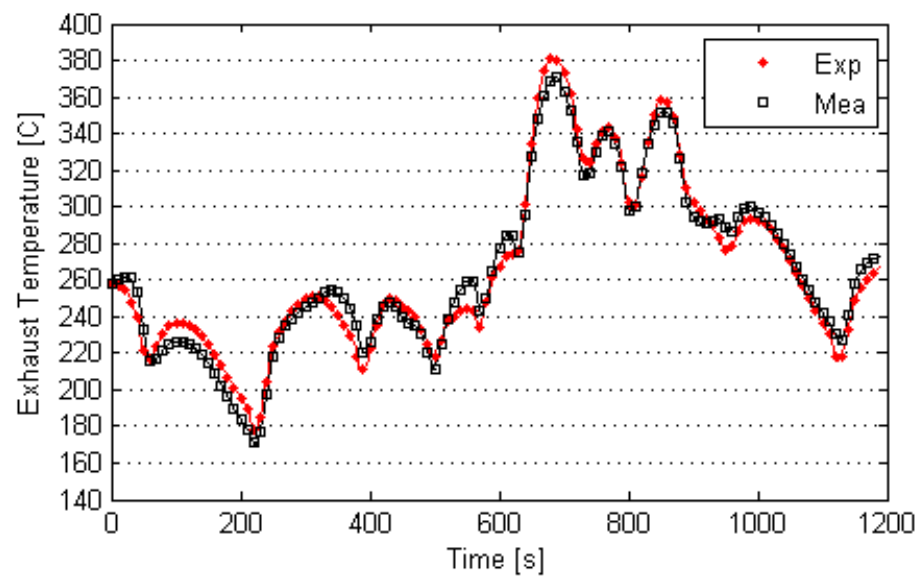

(b) FTP Cycle 2

Figure 6.18: Distribution of computed PM mass within the DPF. 
Results in terms of DOC outlet exhaust temperature are depicted in Figure 6.18 which shows values for both the first and the second FTP cycle. Simulated and measured values appear to be in satisfactory agreement, with a maximum discrepancy of $10.9^{\circ} \mathrm{C}$ measured during the Los Angeles Freeway (LAFY) portion of the FTP cycle. This can be attributed to the percentage of time spent at certain temperatures; in fact, measurements of exhaust temperature downstream the diesel oxidation catalyst reveal that the values are mostly in the temperature window $225-300^{\circ} \mathrm{C}$. This fact has an impact on the calibration process which is based on the minimization of the sum of squares of the errors between measured and simulated data. This fact is depicted in Figure 6.19 which shows the parity plot for the exhaust temperature of FTP cycle 1.

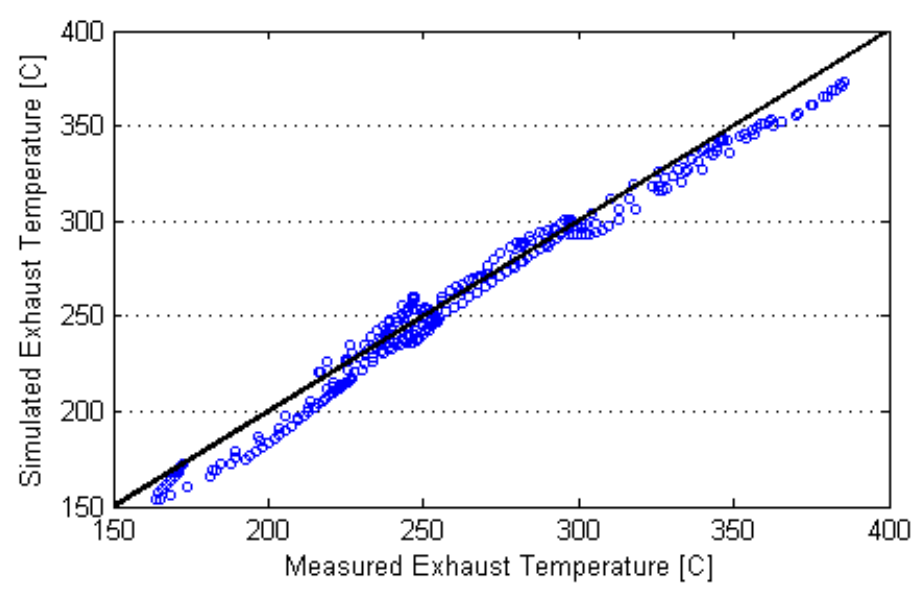

Figure 6.19: Parity plot for engine exhaust temperature downstream the DOC during FTP 1.

Results in terms of species concentrations are reported in terms of several metrics, in a similar manner to what presented in case of the steady-state loading cycle. Given the highly transient nature of the engine cycle, it was of primarily importance to verify if both the DOC models were able to replicate the emissions traces. Comparison between simulated and measured values downstream the diesel oxidation catalyst are provided in Figure 6.20, Figure 6.21 and Figure 6.22.

Results in terms of continuous emissions are in good agreement with the experimental data gathered during the FTP cycle. In particular, the fidelity of the model in reproducing 

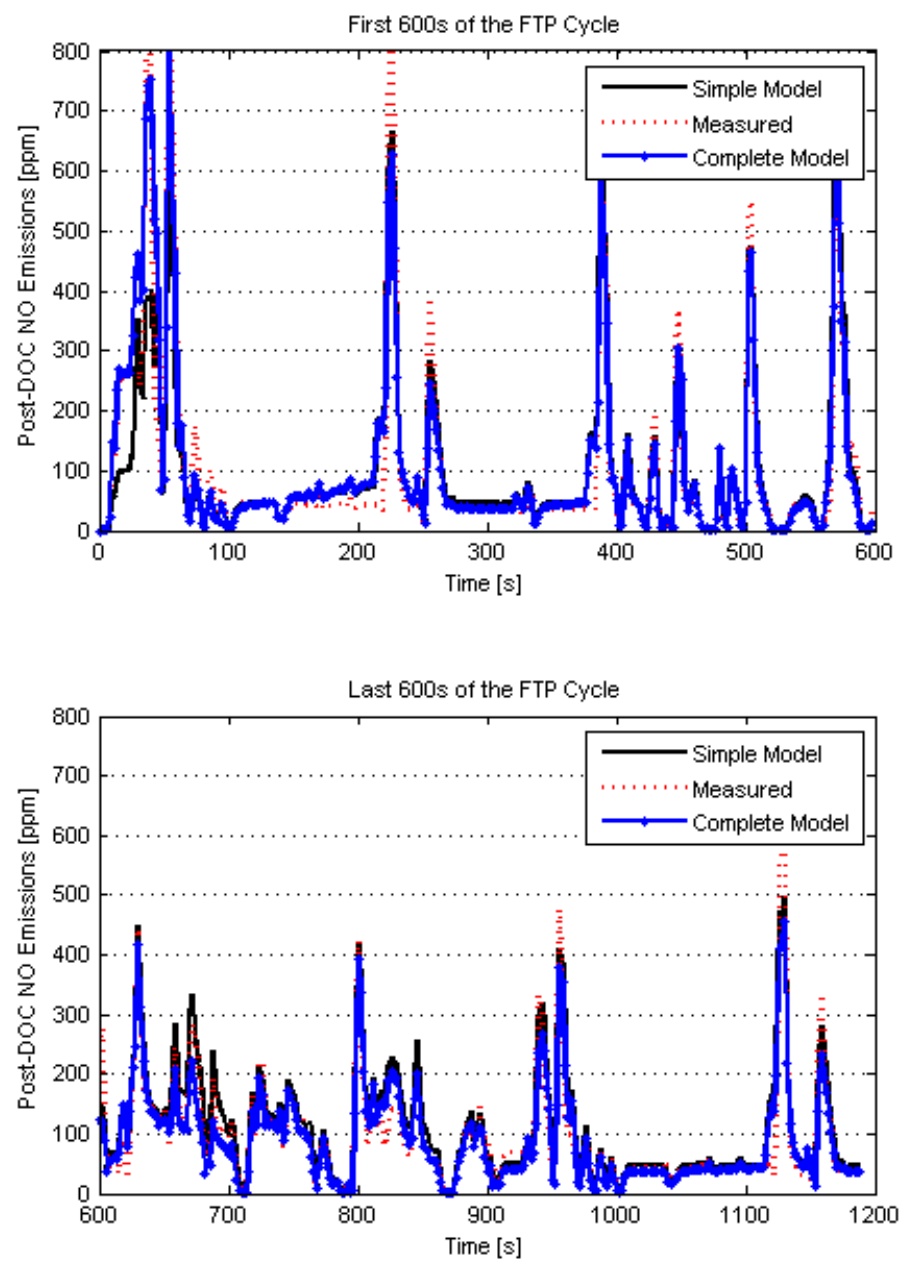

Figure 6.20: Post-DOC NO emissions trace: simulated versus measured values.

NO oxidation appears to be satisfactory at both high and low engine exhaust temperature and flow rate. This suggests that the calibration process although capable of offering only apparent values for key parameters was able to generate a robust model calibration. Further, discrepancies between simulated and measured values appear to be localized in the first $50 \mathrm{~s}$ of the cycle and between $t=650 \mathrm{~s}$ and $t=700 \mathrm{~s}$. Finally, both the simple and complete model struggle to simulate peak events.

The agreement between the two models starts to diminish in case of hydrocarbons results, as depicted in Figure 6.21. At first, it is worth noting that the complete model is capable of reproducing experimental data only on average values. Differently from the measured values which do not appear to fluctuate during transient operations, simulated 


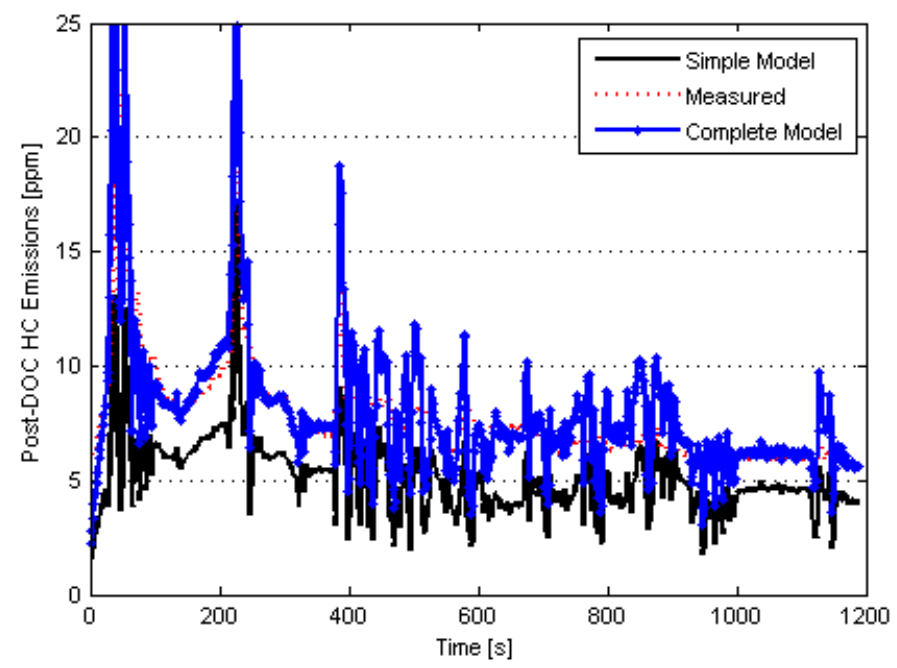

Figure 6.21: Post-DOC HC emissions trace: simulated versus measured values.

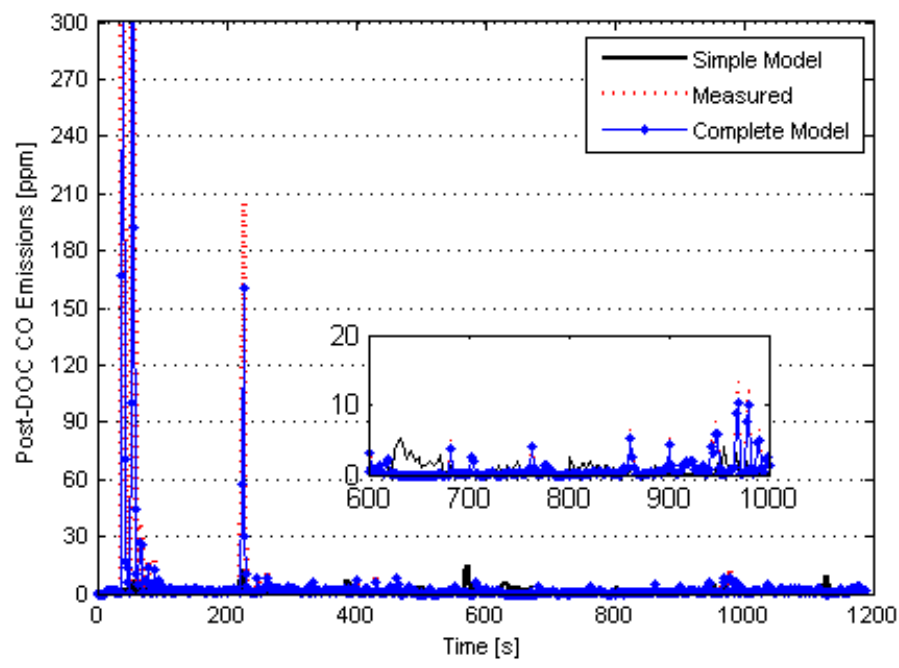

Figure 6.22: Post-DOC CO emissions trace: simulated versus measured values.

values are more noisy suggesting that the effects of mass transfer is overestimated in the models. Further, in case of the simple model, results appear to be scaled by a 1.8 factor. The discrepancy between the two models can be explained considering the different level of detail used to implement and solve the equations for species conservation. Simulated values appear to be in good agreement with experimental results also in case of carbon monoxide. The trend observed during the steady-state cycle with outlet concentrations of the range of 1-2 ppm is present also in case of the FTP cycle. It is worth noting that results from the complete model show good agreement also during peak events. 
From analysis of results in terms of continuous traces it can be concluded that the DOC model is capable to replicate NO oxidation with good accuracy, with discrepancies that are limited to peak events. The limits of the model start to appear in case of hydrocarbons where the signal generated by the model is characterized by a higher level of noise. This could be due to a calibration that results in a model particularly sensitive to quick changes in exhaust flow and therefore in mass transfer efficiencies. Further, poor speciation of total hydrocarbons, modeled as pure $C_{3} H_{6}$ in this study, may represent an additional limiting factor.

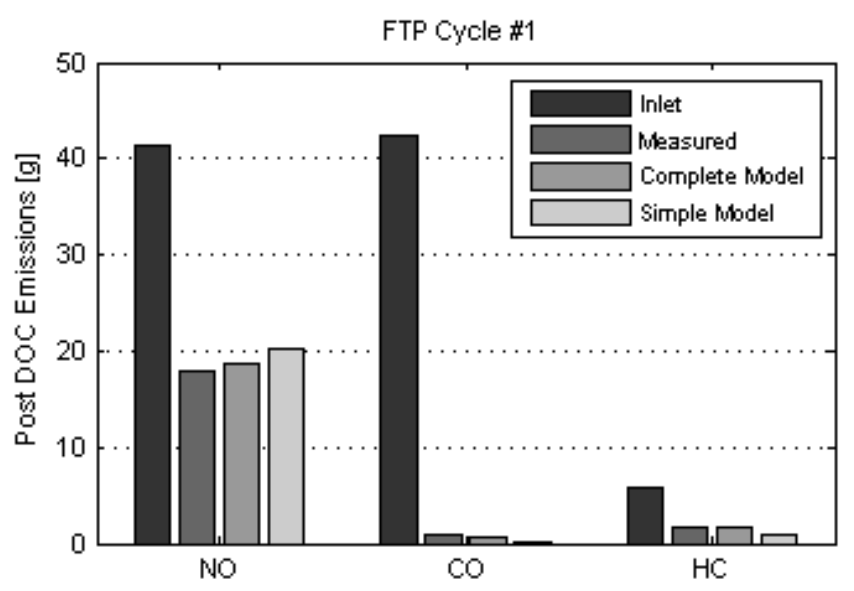

(a) FTP Cycle 1

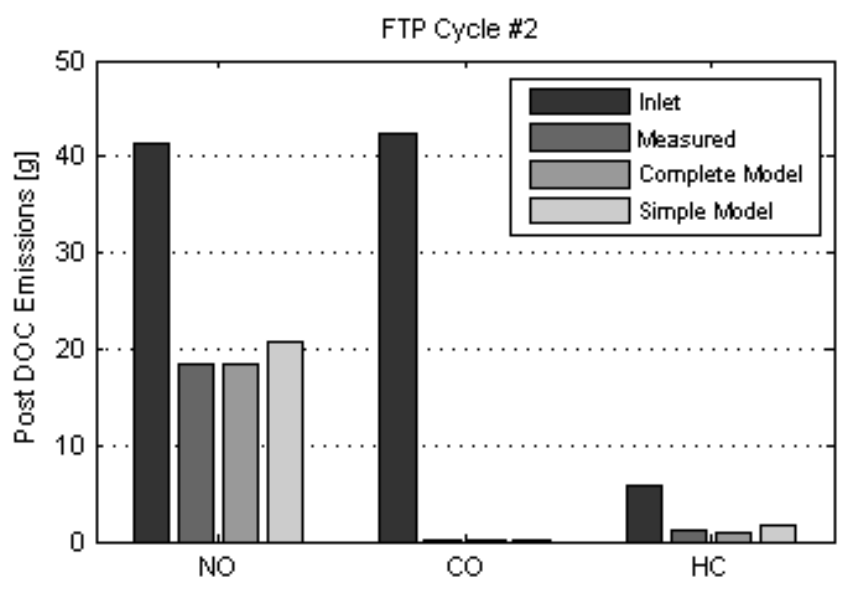

(b) FTP Cycle 2

Figure 6.23: Total Mass Emissions over FTP Cycle: Simulated versus Measured Values

Comparison between simulated and measured results in terms of total mass emissions over the FTP cycle are provided in Figure 6.23. In case of $N O$ emissions, the complete model $(18.7 \mathrm{~g})$ is able to replicate experimental results $(17.92 \mathrm{~g})$ with a relative error of 
$+4.3 \%$ whilst the discrepancy of the simple model $(20.29 \mathrm{~g})$ results to be of $+13.22 \%$.

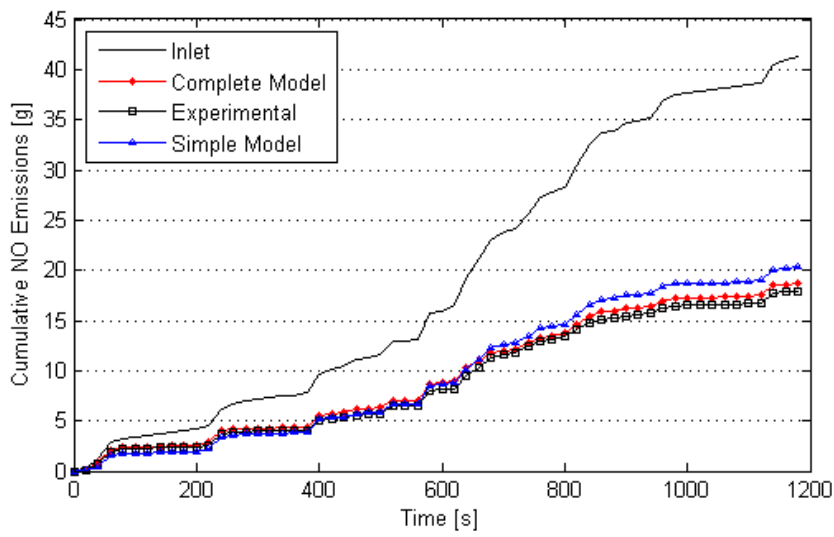

(a) NO Mass Emissions

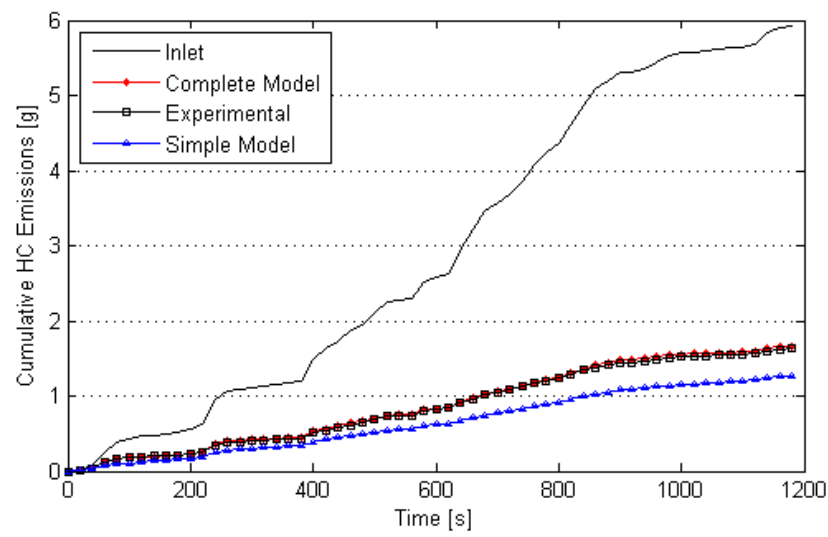

(b) HC Mass Emissions

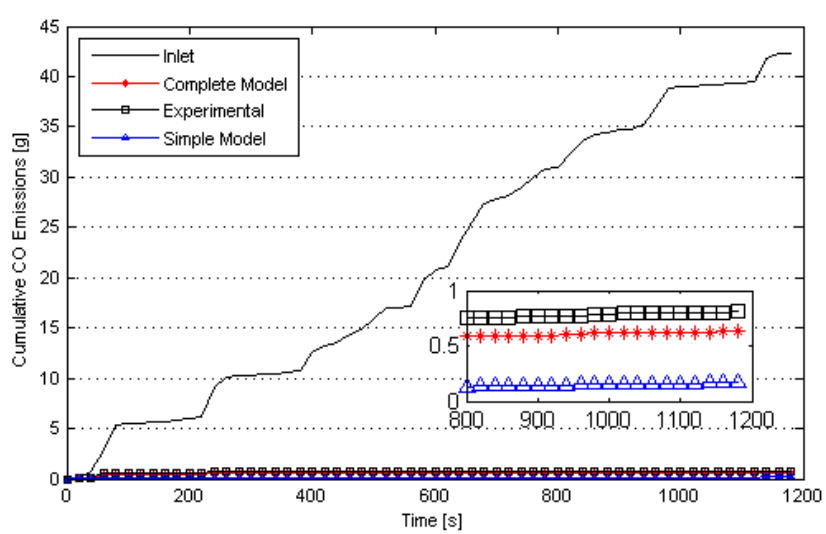

(c) CO Mass Emissions

Figure 6.24: Cumulative mass emissions over the FTP cycle: comparison between simulated and measured values.

Results in terms of hydrocarbons show that the complete model $(1.67 \mathrm{~g})$ estimates total mass emissions within a $+2.45 \%$ error while the error of the simple model $(0.97 \mathrm{~g})$ reaches 
$-40.4 \%$. In case of carbon monoxide emissions the discrepancies from the experimental measurements are $-22 \%$ and $-74 \%$ for the complete and simple model respectively. Finally, cumulative mass emissions over the FTP cycle are depicted in Figure 6.24 where they are compared to the total mass entering the diesel oxidation catalyst. Also, computational time for the complete model was $48.6 \mathrm{~s}$ while the simplified model was capable of replicating a full FTP cycle in $12.3 \mathrm{~s}$.

\subsubsection{DPF Model}

The DPF model used to validate experimental results gathered during the loading cycle was used to predict filter pressure drop and filter PM mass content over the FTP cycle. The tuning parameters commented in Chapter 5 (Table 5.1), were kept constant as the capabilities toward real-time applications had to be demonstrated in this context. From a first inspection of the input data depicted in Figure 6.17, it appears evident that during the $L A F Y$ section of the FTP cycle, the highest exhaust flow rate and temperature conditions occur thus giving the best conditions over the cycle in terms of regeneration potential.

Results in terms of filter pressure drop are provided in Figure 6.25 which shows satisfactory agreement between the experimental and the simulated values. It is worth noting that the DPF model is capable of replicating the measured pressure drop during both heavily transient regimes with high degree of precision. Further, good agreement is achieved also during idle conditions which, at the contrary, are characterized by low values of exhaust flow. In this regard, two aspects play a major role in determining the accuracy of model and therefore need to be correctly estimated: the temperature of the substrate wall and the amount of PM mass trapped in the filter. The effect of the temperature on filter pressure drop can be observed in Figure 6.25a noting that the two New York Non-Freeway (NYNF) portions of the FTP cycle, identical in terms of engine load and speed, give almost $50 \%$ difference in terms of pressure drop. 


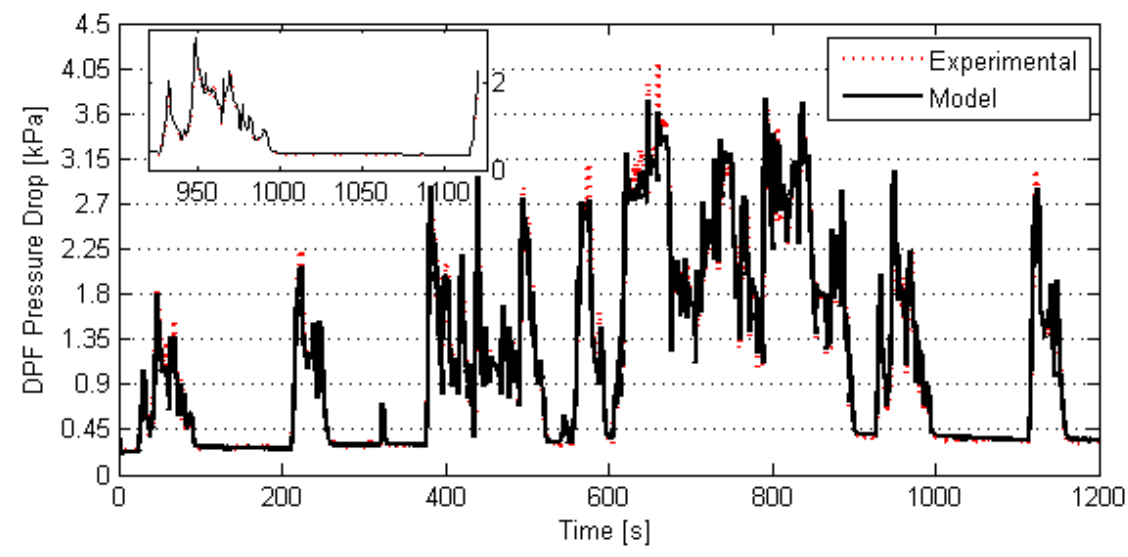

(a) FTP Cycle 1

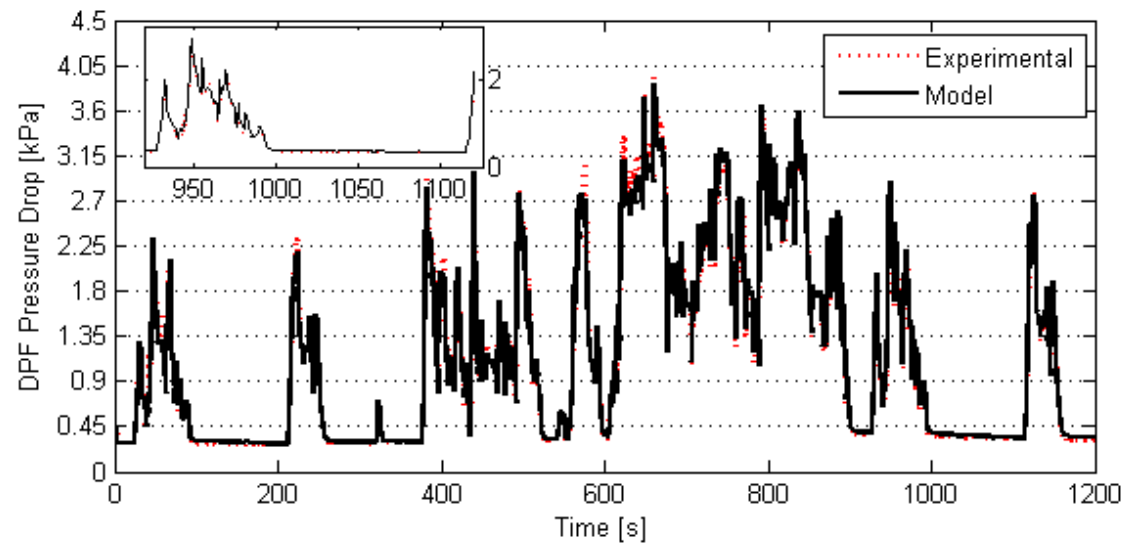

(b) FTP Cycle 2

Figure 6.25: Filter pressure drop during the FTP Cycle: simulated versus measured values.

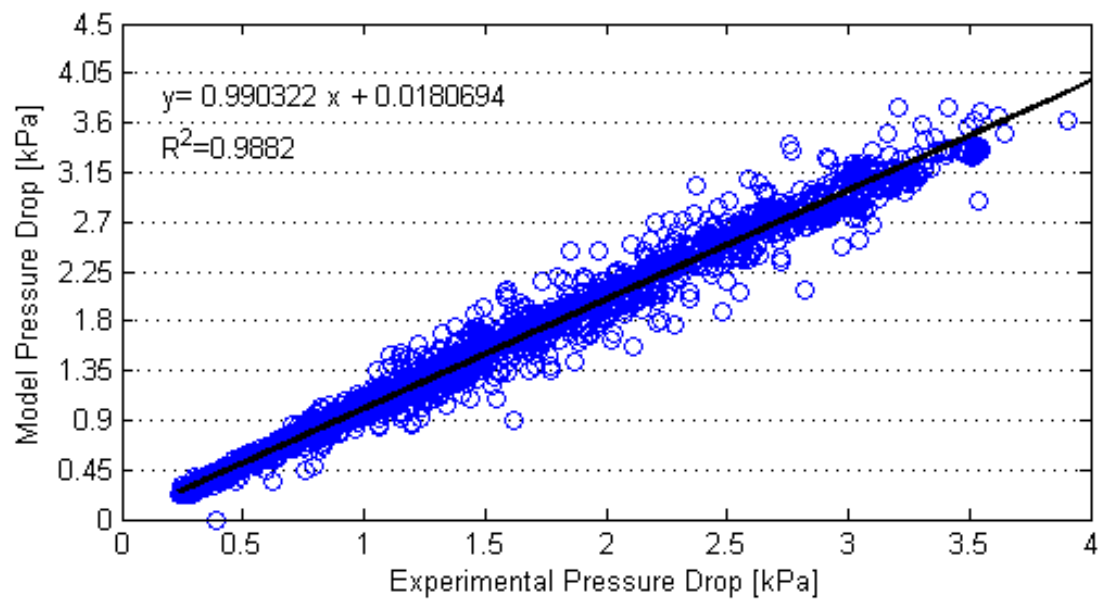

Figure 6.26: Simulated and measured filter pressure drop: parity plot.

Figure 6.27 shows a comparison between the experimental exhaust temperature measured downstream of the DPF and the computed substrate temperature. Discrepancies 
between the two values appear to be significant only during the first $400 \mathrm{~s}$ of the FTP cycle where the error reaches value of the order of $30^{\circ} \mathrm{C}$, especially in case of the second FTP (hot start). However, results appear to be satisfactory considering the 1-D nature of the DPF model and the simplifying hypotheses assumed in this study.

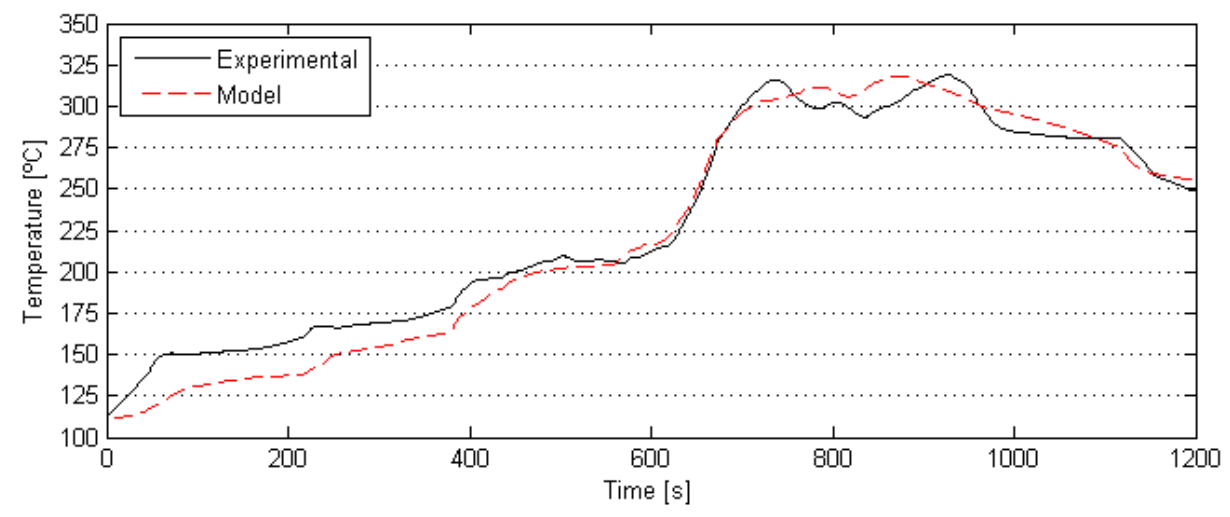

(a) FTP Cycle 1

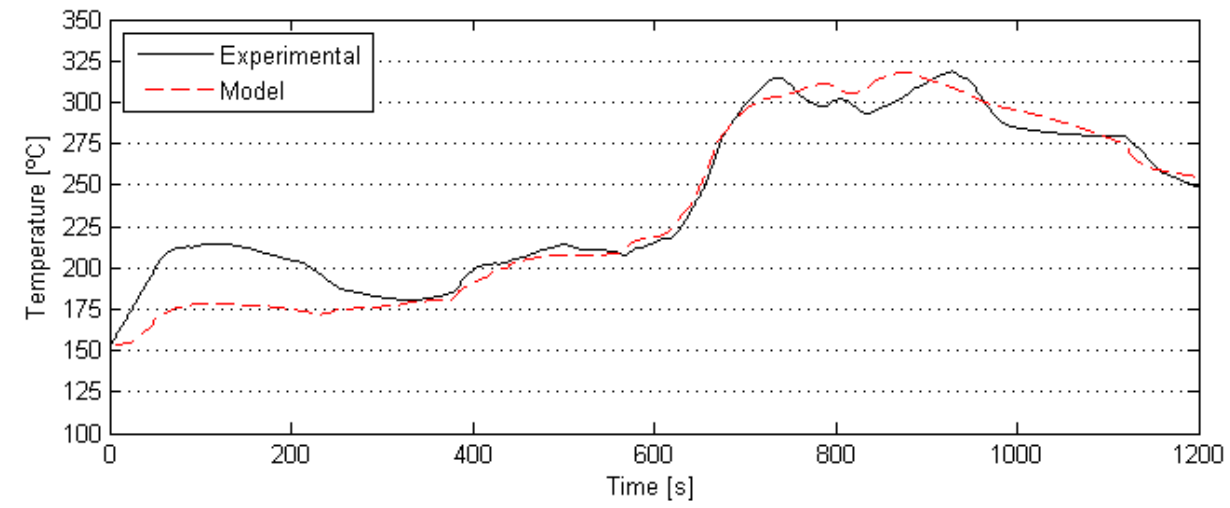

(b) FTP Cycle 2

Figure 6.27: Modeled substrate temperature versus measured post-DPF exhaust temperature over the FTP cycle

Model outputs in terms of PM loading during the two FTP cycles are depicted in Figure 6.28 which shows the mass balances for DPF. Results are plotted against two yaxes, with the "Trapped mass" curve referring to the right axes whilst "Oxidized mass" and "Inlet mass" refers to the left axes. Values reported in Figure 6.28 are restricted to the FTP cycles, meaning that the depicted cumulative mass values refer just to that specific engine cycle. With reference to Figure 6.28a, analysis of the results shows that during the FTP the engine produces a total of $1.293 \mathrm{~g}$ of PM mass, computed by integration of the 
signal recorded by the AVL Soot-Sensor. Further, comparison between the three curves shows that all of the PM mass entering the DPF is initially trapped in the cake layer, with PM loading reaching the maximum value of $0.74 \mathrm{~g}$ at $t=674 \mathrm{~s}$. After this point in time, soot oxidation starts to become significant due to the higher exhaust temperatures characterizing the second half of the FTP cycle. In this regard, it is worth noting that the oxidation rate is faster than the rate of soot entering the DPF. This ultimately results in oxidation of part of the PM mass accumulated during the loading cycle leading to the negative values for the "Trapped mass".

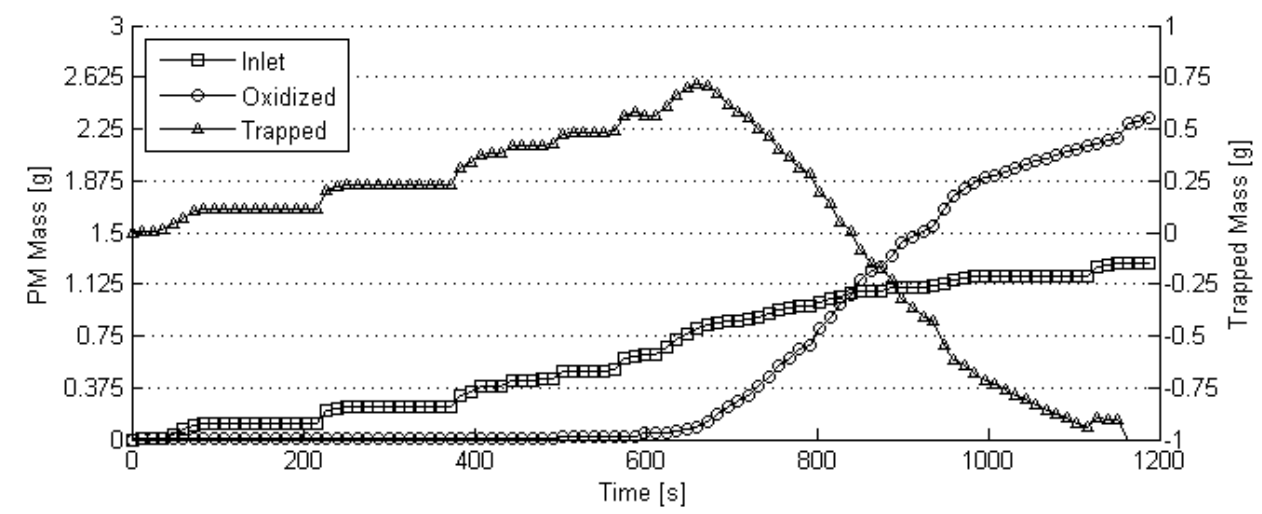

(a) FTP Cycle 1

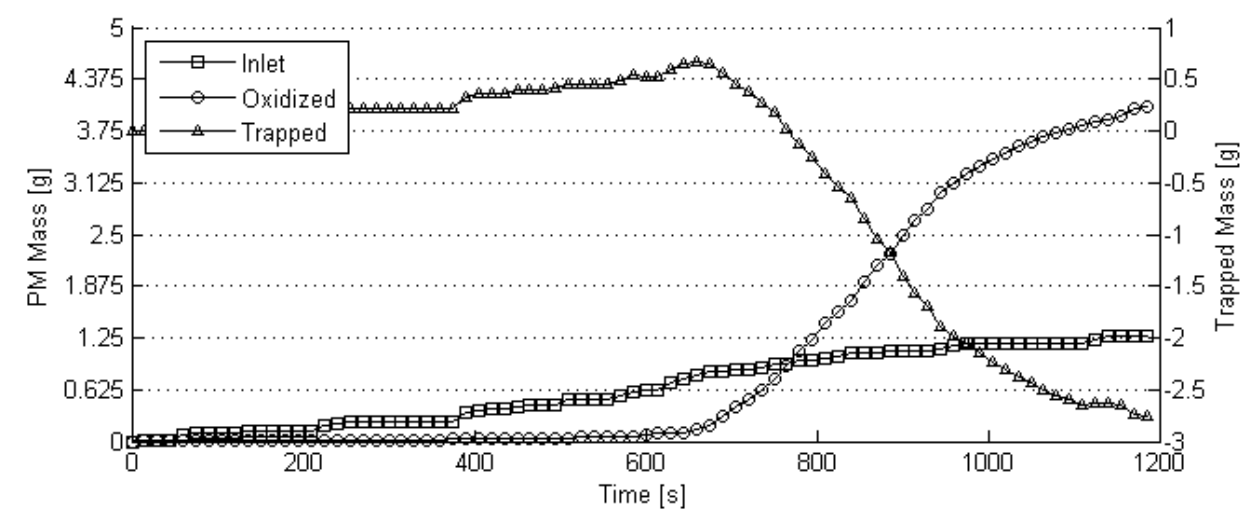

(b) FTP Cycle 2

Figure 6.28: Filter mass balance over the FTP cycles.

Figure 6.29 shows the same results in terms of thickness of the cake layer. It is worth noting that the red horizontal line depicts the value of the soot layer thickness at the beginning of each FTP cycle whilst the vertical arrow on the right side of the figures points to the final thickness of the soot layer as an indication of the amount of layer 
length that was depleted during the oxidation process. As discussed during the analysis of the results of the loading cycle, the status of the filter is such that $99 \%$ of the trapped mass is deposited as soot layer on top of the substrate wall. Further, the oxidation process is more efficient in this location due to the presence of more PM mass when compared to the filter substrate.

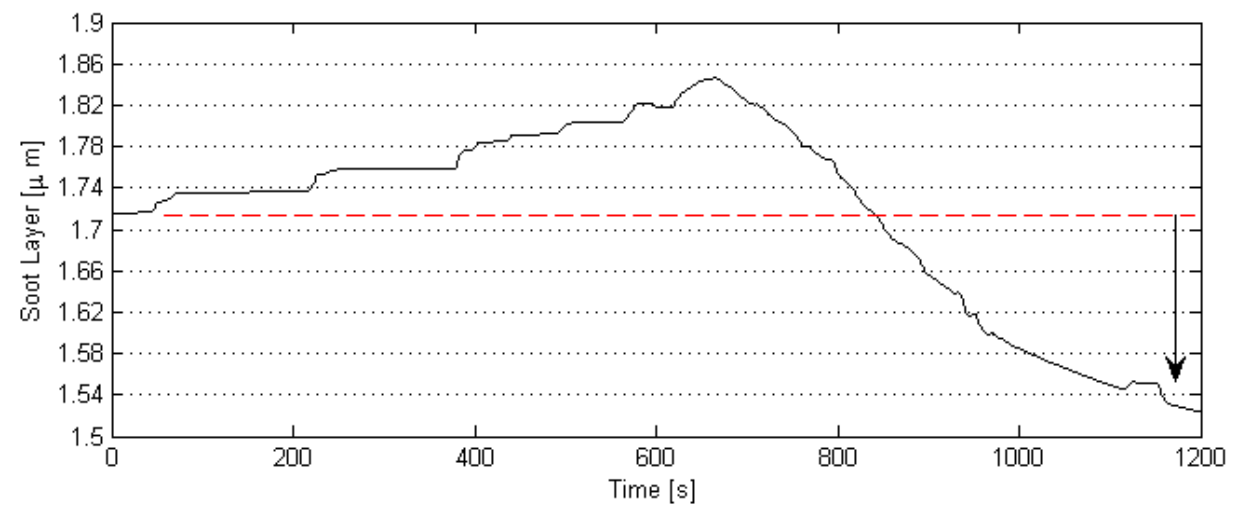

(a) FTP Cycle 1

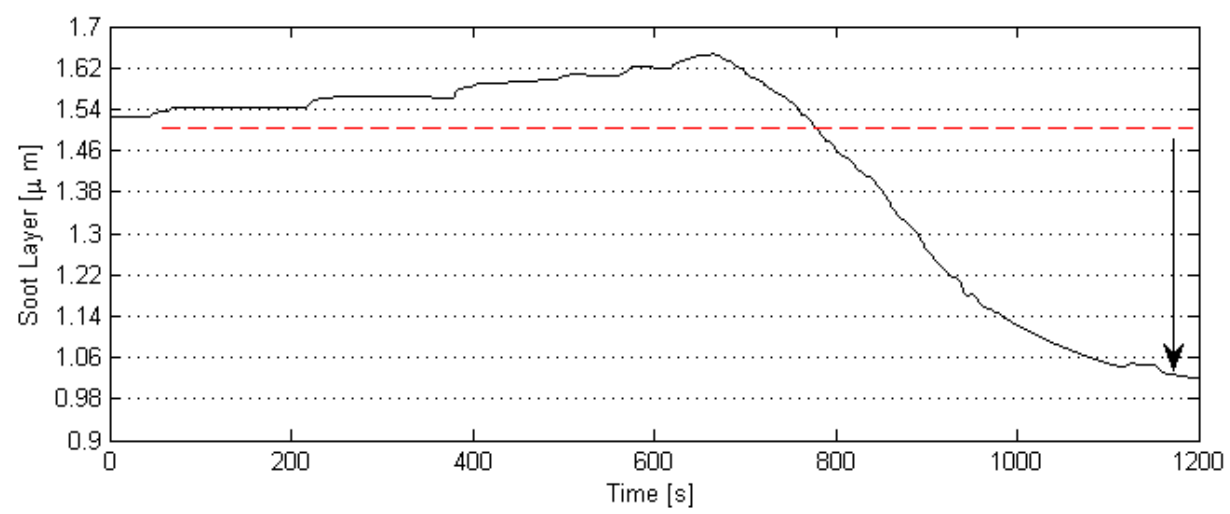

(b) FTP Cycle 2

Figure 6.29: Evolution of soot layer thickness during the FTP cycle.

To conclude this section, analysis of the results gathered during the FTP cycles indicate that the DPF model can be employed to simulate transient operating conditions. Results in terms of filter pressure drop show very good agreement with experimental results for two consecutive FTP cycles, suggesting that dynamics taking place within the filter are captured. In this regard, simulated values for PM mass balance indicate that the FTP is, on average, a regenerative engine cycle. This fact is due to the high exhaust flow rates and temperature generated by the engine during the $L A F Y$ portion of the FTP. 


\subsection{DPF Regeneration Cycle}

\subsubsection{Engine Performance and Emissions}

Results in terms of engine performance and average brake specific emissions for the R100 engine cycle are presented in Table 6.5. Consistently with what presented for the case of loading and FTP cycles, exhaust emissions were also sampled from the raw exhaust (not-diluted) and results in terms of concentrations are provided in Table 6.6 at three different locations over the aftertreatment system.

Table 6.5: Average brake specific emissions for the R100 engine cycle.

\begin{tabular}{lcc}
\hline \hline Parameter & Value & Units \\
\hline EngineSpeed & 1800 & $\mathrm{rpm}$ \\
EngineTorque & 1018 & $\mathrm{ft}-\mathrm{lb}$ \\
FuelRate & 15.51 & $\mathrm{~g} / \mathrm{s}$ \\
$\mathrm{HC}$ & $8.0610^{-4}$ & $\mathrm{~g} / \mathrm{bhp}-\mathrm{hr}$ \\
$\mathrm{NO}_{x}$ & 1.618 & $\mathrm{~g} / \mathrm{bhp}-\mathrm{hr}$ \\
$\mathrm{CO}$ & $3.710^{-4}$ & $\mathrm{~g} / \mathrm{bhp}-\mathrm{hr}$ \\
$\mathrm{CO}_{2}$ & 476.4 & $\mathrm{~g} / \mathrm{bhp}-\mathrm{hr}$ \\
\hline \hline
\end{tabular}

Table 6.6: Average raw emissions measured over the R100 cycle.

\begin{tabular}{lccc}
\hline \hline Pollutant & Pre-DOC & Pre-DPF & Post-DPF \\
\hline$H C[p p m]$ & 38.3 & 7.9 & 5.8 \\
$C O[p p m]$ & 389.2 & 1.1 & - \\
$N O_{x}[p p m]$ & 487.4 & 486.2 & 488.6 \\
$N O[p p m]$ & 438.3 & 379.4 & 370.2 \\
$N O_{2}[p p m]$ & 48.7 & 107.6 & 113.3 \\
$P M\left[\mathrm{mg} / \mathrm{m}^{3}\right]$ & 11.14 & - & - \\
\hline \hline
\end{tabular}

It is worth noting that although the $N O_{x}$ concentration is considerably higher than that observed during the R10 mode, the $N O / N O_{x}$ ratio downstream of the diesel oxidation catalyst is still on values of the order of $77 \%$. In terms of $N O$ conversion efficiency, comparison between experimental values gathered during loading and regeneration cycles 
shows a drop from $58.8 \%$ to $13.5 \%$ in case of mode R100. This suggests that the diesel oxidation catalyst is operating in equilibrium limited regime, as far as the oxidation of nitric oxide is concerned. As long as particulate matter emissions are concerned, characterization of the particle size distribution is provided in Figure 6.30.

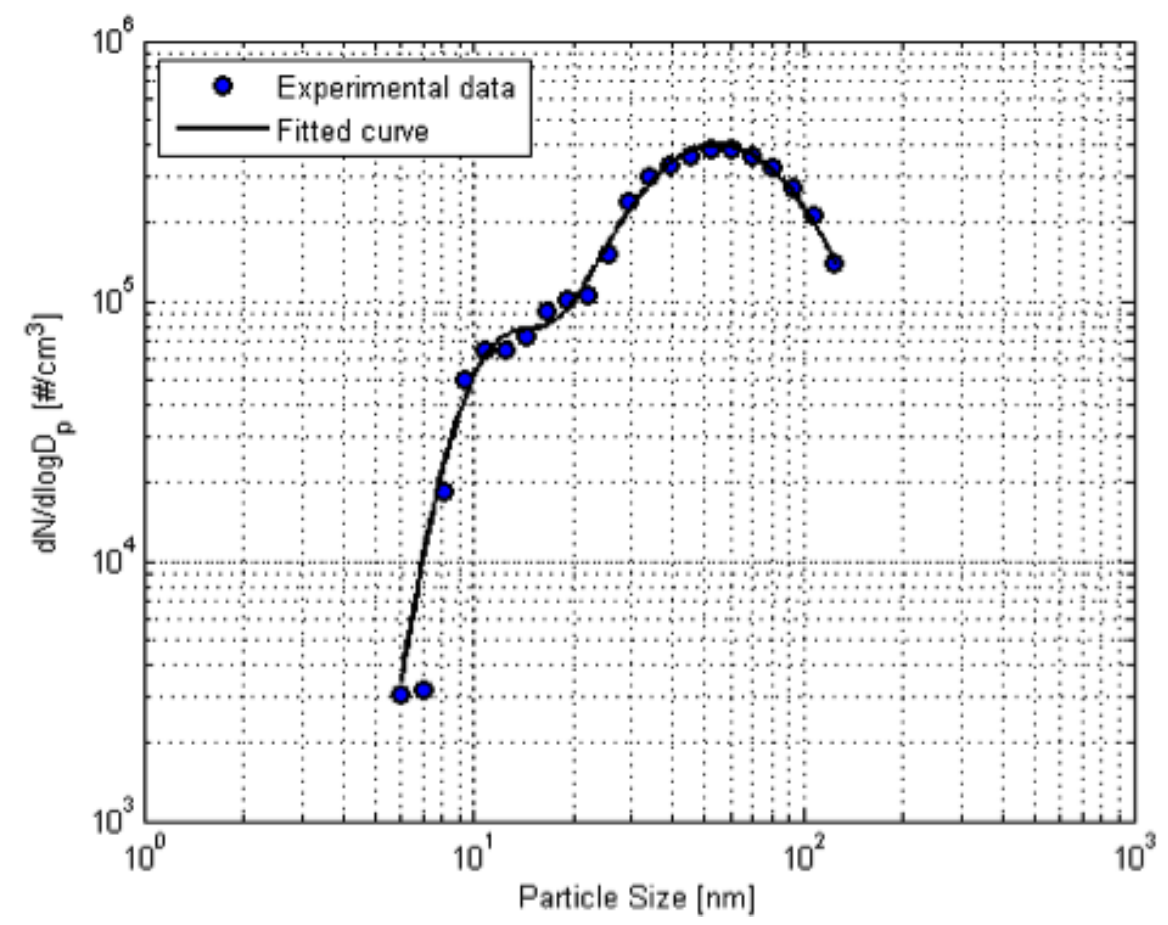

Figure 6.30: Time averaged particle size distribution entering the C-CRT over the R100 cycle.

\subsubsection{DOC Model}

During a filter regeneration mode, the diesel oxidation catalyst has to ensure that a) the exhaust temperature entering the DPF is sufficient to support soot oxidation (this is required in case of active regenerations) and b) create the optimal conditions in terms of $\mathrm{NO} / \mathrm{NOx}$ ratio to enhance soot oxidation by $\mathrm{NO}_{2}$. Although oxidation of diesel fuel was implemented in the DOC model, this tool could not be tested as the experimental apparatus used in this study was a passively regenerating trap. Results in terms of outlet exhaust temperature are provided in Figure 6.31; deviations of simulated values from experimental results are of the order of $1^{\circ} \mathrm{C}$. 


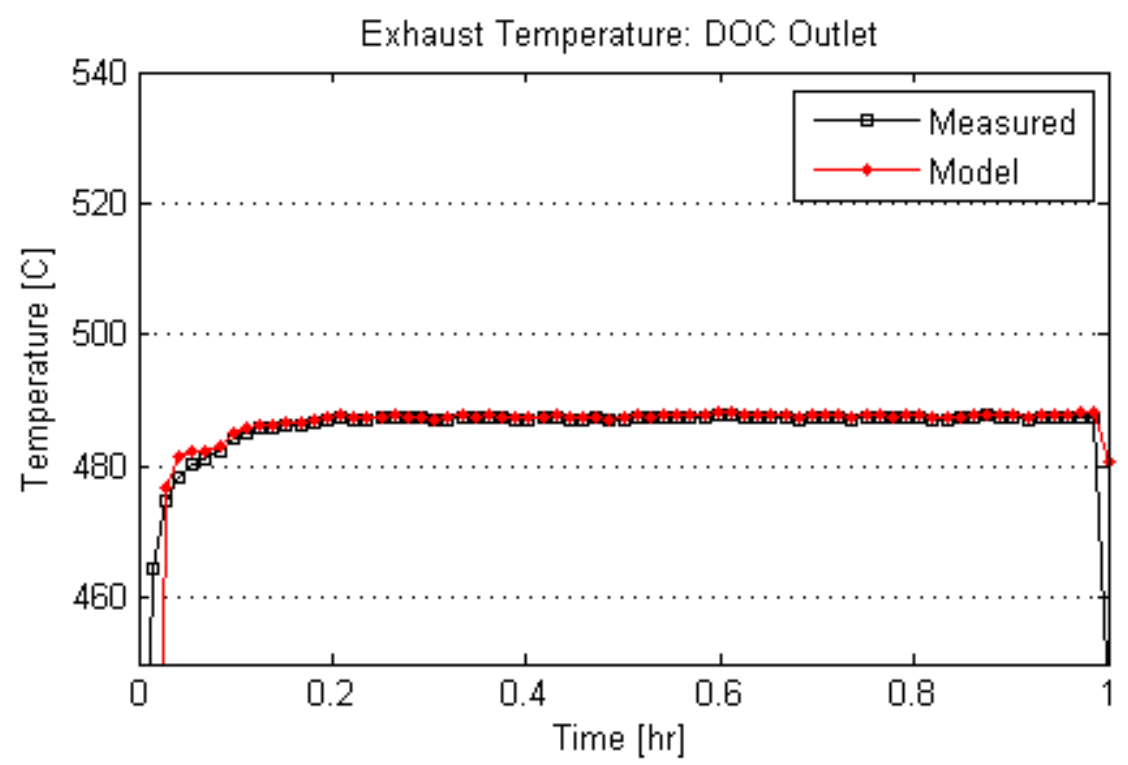

Figure 6.31: Post-DOC exhaust temperature: measured versus simulated results.

Time averaged concentrations in the raw exhaust (not-diluted) are listed in Table 6.7 which offers a comparison between simulated and measured values.

Table 6.7: Simulated and measured average raw concentrations downstream the DOC over the R100 cycle.

\begin{tabular}{lccc}
\hline \hline Pollutant & Complete Model [ppm] & Simple Model [ppm] & Measured [ppm] \\
\hline HC & 9.8 & 6.5 & 7.9 \\
$\mathrm{CO}$ & 0.43 & 0.26 & 0.52 \\
$\mathrm{NO}$ & 383 & 374.5 & 379 \\
\hline \hline
\end{tabular}

Also, in the case of the R100 mode, experimental and simulated results appear to be in satisfactory agreement with raw exhaust concentrations being estimated within a $10 \%$ error. Results in terms of continuous traces are depicted in Figure 6.32.

It should be noted that $N O$ concentrations estimated by the DOC models are in agreement with experimental values suggesting that limitations due to thermodynamic equilibrium are correctly captured by the models. The equilibrium limited conversion of $N O$ can be appreciated in Figure 6.33 which shows the evolution of the inlet concentrations over the length of the diesel oxidation catalyst. It is worth noting that $N O$ concentration 

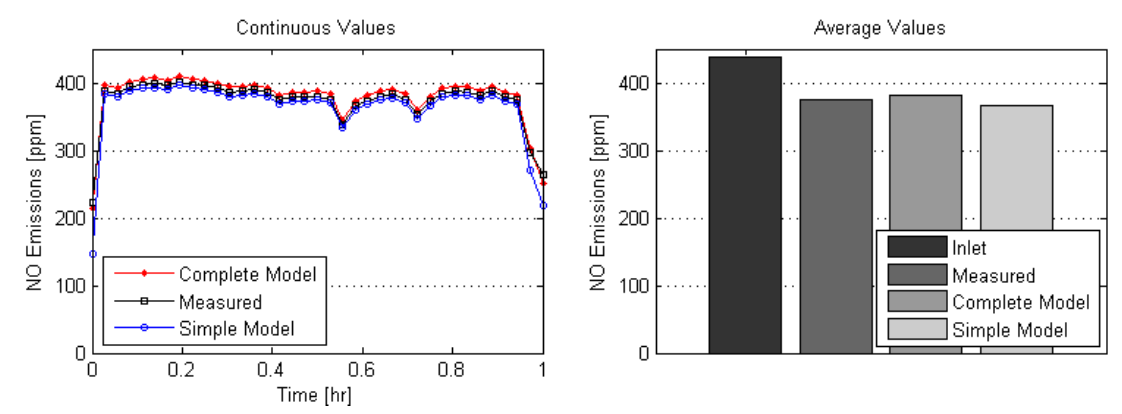

(a) Nitric Oxide
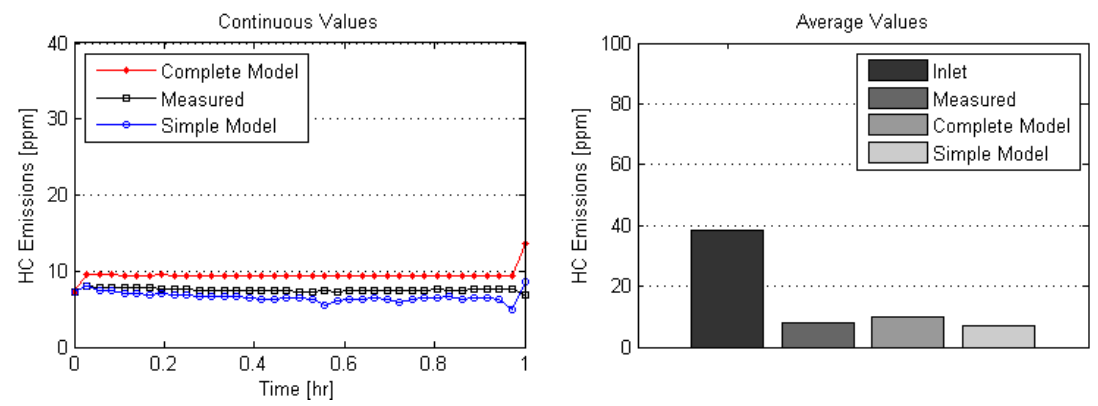

(b) Hydrocarbons
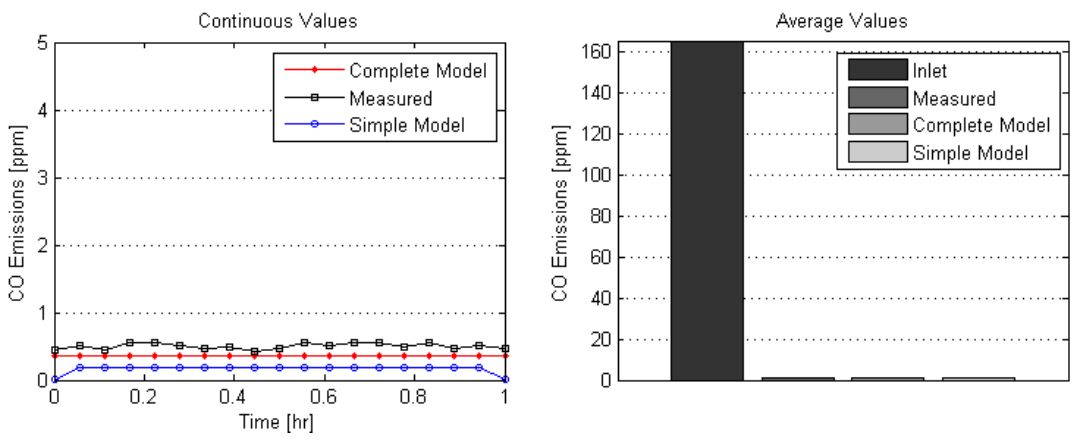

(c) Carbon Monoxide

Figure 6.32: Continuous emissions traces during the R100 cycle.

initially decrease reaching a minimum value at $x / L=0.3$. At this location the thermodynamic conditions within the DOC channel are such that part of the $\mathrm{NO}_{2}$ is converted back to $N O$ and the system reaches the equilibrium at $x / L=0.4$. This behavior is completely different from what observed during the loading cycle (R10) where inlet $N O$ concentrations decrease continuously to reach a minimum value at the outlet of the DOC.

The good agreement between modeled and measured values is maintained in the computation of cumulative mass emissions as well. Figure 6.34 depicts the cumulative mass for $N O, H C$ and $C O$ emitted over the R100 mode. Measured values are listed in Table 6.8. In case of nitric oxide emissions, the discrepancy between simulated and measured 


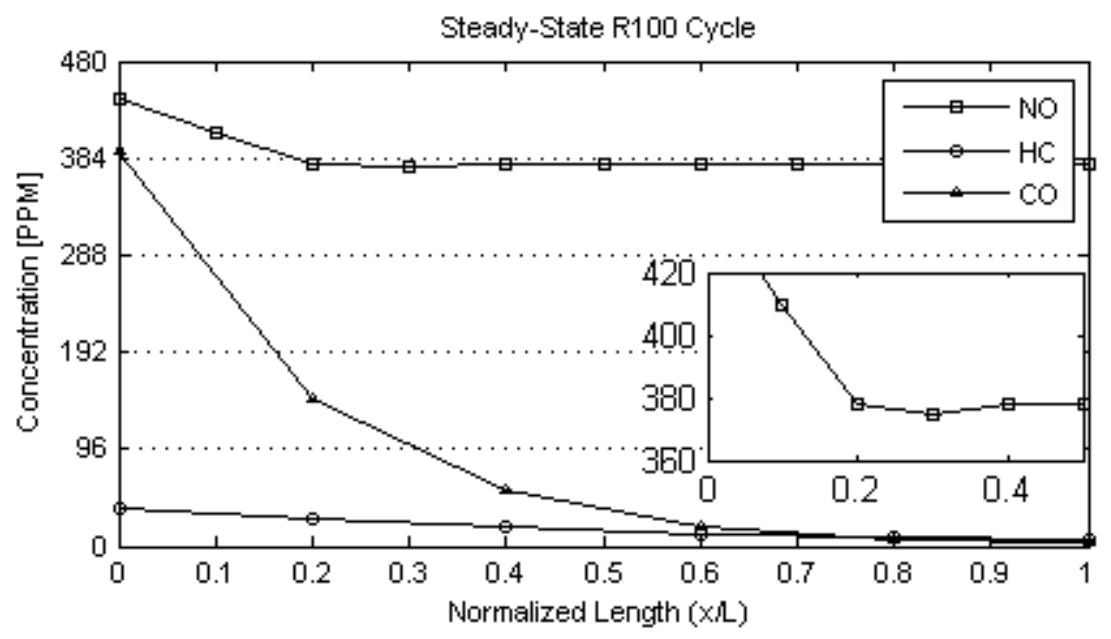

Figure 6.33: Evolution of inlet concentrations over the length of the DOC.

values is limited to $-1.98 \%$ and $+2.03 \%$ for the simple and complete models, respectively. Similarly to what was observed in case of the R10 and FTP cycles, deviations of simulated values from experimental results approach larger values in case of hydrocarbons; the relative error for the complete and simple models are $+27.4 \%$ and $-10.4 \%$, respectively.

Table 6.8: Measured mass emissions over the R100 mode.

\begin{tabular}{lcc}
\hline \hline Gaseous Specie & Value & Units \\
\hline$H C$ & 12.42 & $\mathrm{~g}$ \\
$N O$ & 444.7 & $\mathrm{~g}$ \\
$\mathrm{CO}$ & 0.54 & $\mathrm{~g}$ \\
\hline \hline
\end{tabular}

Finally, results in terms of carbon monoxide show that the descrepancy between simulated and measured values are $-29.86 \%$ and $-63.54 \%$ for the complete and simple models, respectively. In agreement with what was presented earlier in this chapter, both DOC models appear to capture the main phenomena taking place in the diesel oxidation catalyst providing estimations characterized by an accuracy which is species dependent. In case of nitric oxide, model estimations are always within a $15 \%$ from measured values whilst in case of both hydrocarbons and carbon monoxide, simulated values appear to deviate more from the experimental results. The reasons for descrepancies are attributed to the simple speciation of the hydrocarbons (treated in this study as pure $C_{3} H_{6}$ ) and 
the very low concentrations of carbon monoxide measured downstream the DOC.
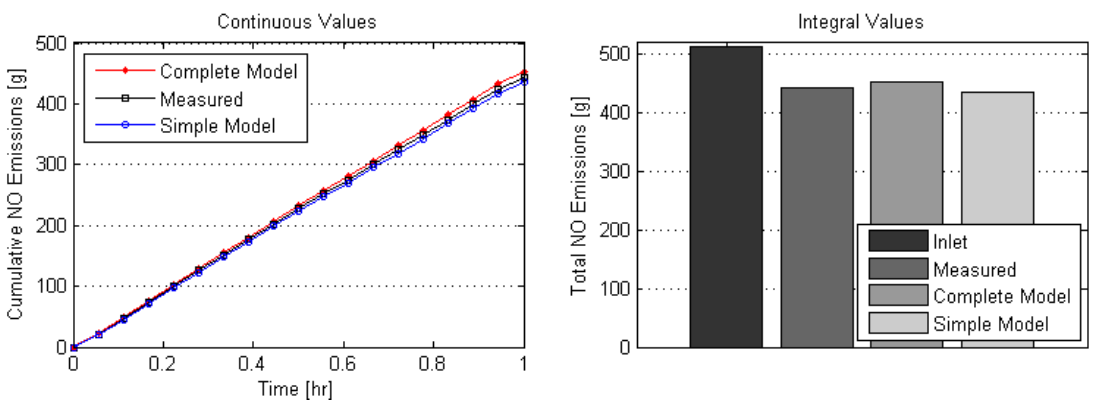

(a) Nitric Oxide
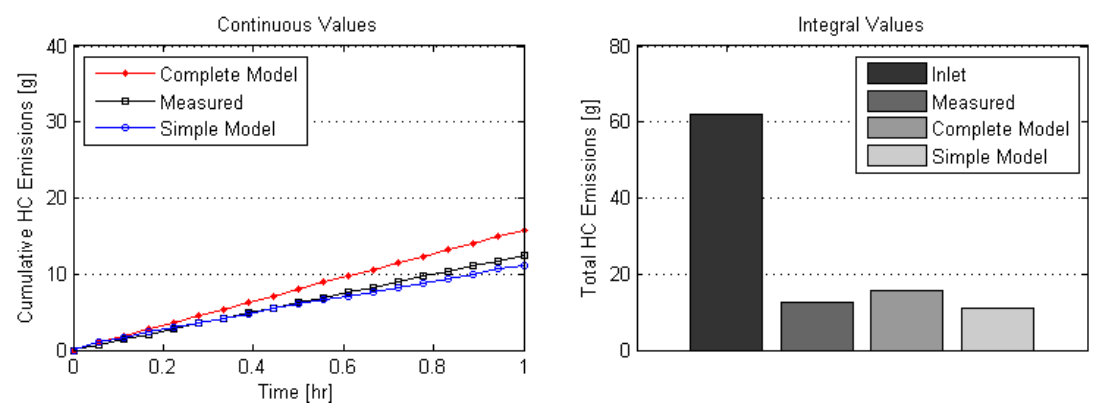

(b) Hydrocarbons
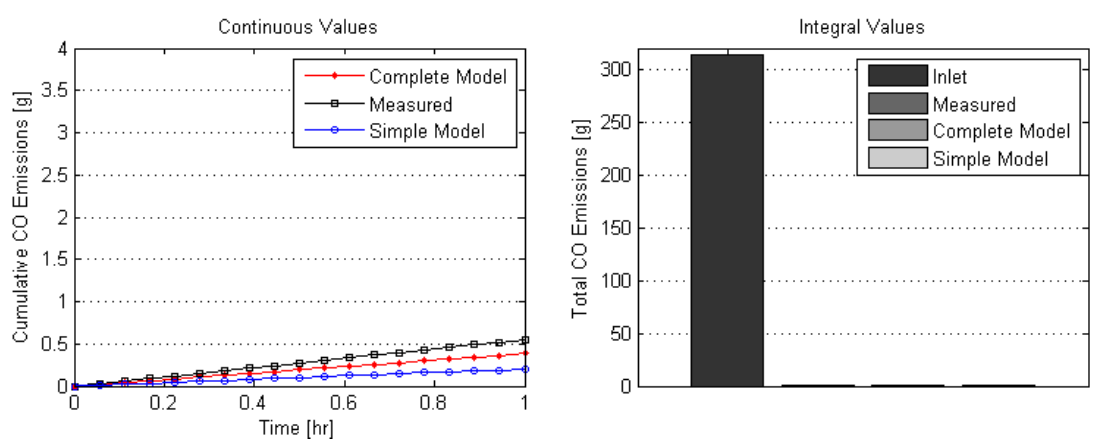

(c) Carbon Monoxide

Figure 6.34: Cumulative mass emissions over the R100 cycle.

\subsubsection{DPF Model}

Results obtained during the regeneration mode reveal a totally different behavior with respect to what it was presented in case of R10 and FTP cycles. At first, the potential for soot loading is much higher at R100 mode as the inlet total mass reaches a value of $4.2 \mathrm{~g}$ in just $1 \mathrm{hr}$. With reference to Figure 6.35, it is worth noting that catalytic and thermal regenerations pathways allow not only to oxidize the PM mass previuosly trapped by the DPF ( $6 g$ after the two FTPs), but also the entire amount of PM entering the DPF 
during the regeneration process itself. This is translated into an oxidation rate which is initially higher than the rate at which PM enters the filter (first $10 \mathrm{~min}$ ) and then reaches an equilibrium (parallel lines in Figure 6.35). The higher PM concentration entering the $\mathrm{DPF}$ is one of the reasons for the small amount of mass left inside the filter at end of the regeneration process; it can be noted that the line representing "Trapped Mass" does not reach a null value although the operating temperature reaches $476^{\circ} \mathrm{C}$ (Figure 6.36). Weighing of the DPF provided a measured value for the soot load of $0.53 \mathrm{~g} / \mathrm{l}$, whilst the computed soot load at the end of the regeneration cycle was of $0.073 \mathrm{~g} / \mathrm{l}$.

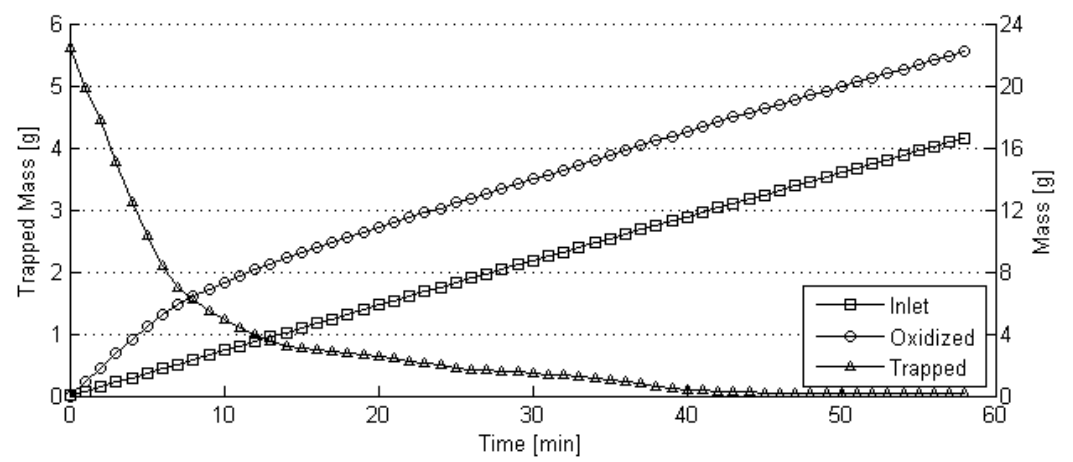

Figure 6.35: Filter mass balance during the regeneration process.

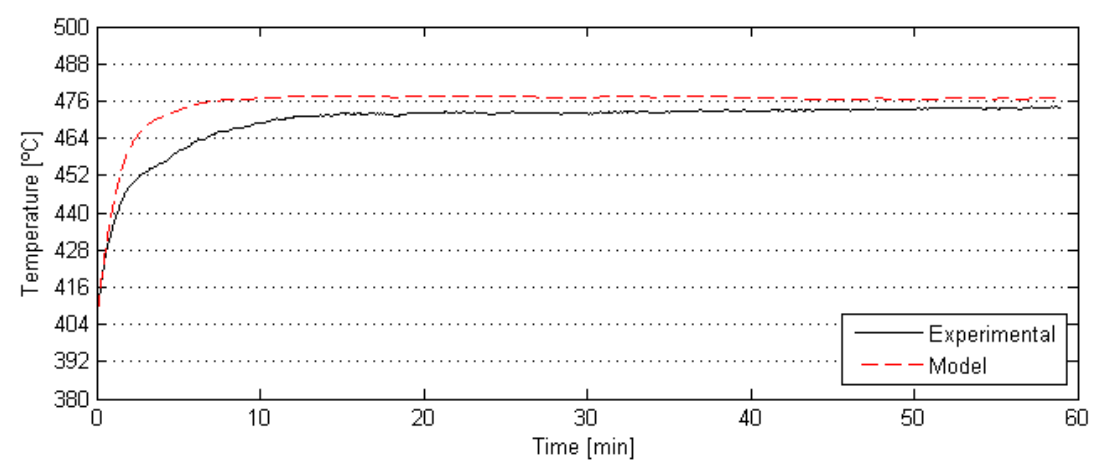

Figure 6.36: DPF substrate temperature versus measured outlet exhaust temperature over the R100 mode.

Characteristics of the oxidation process taking place within the PM filter are depicted in Figure 6.37 which shows results in terms of two parameters: the cake layer on top of the substrate wall and the virtual soot layer per unit thickness defined in Chapter 3. Analysis of Figure 6.37 allows for some considerations. At first, it is worth noting how 
the cake layer reaches an equilibrium condition which differs from $w_{s}=0$, in agreement with the residual trapped mass discussed earlier. Further, a residual layer of soot on top of the substrate wall is also in agreement with the hypothesis of conditioned filter and the formation of a soot membrane after filter loading/regeneration cycles.

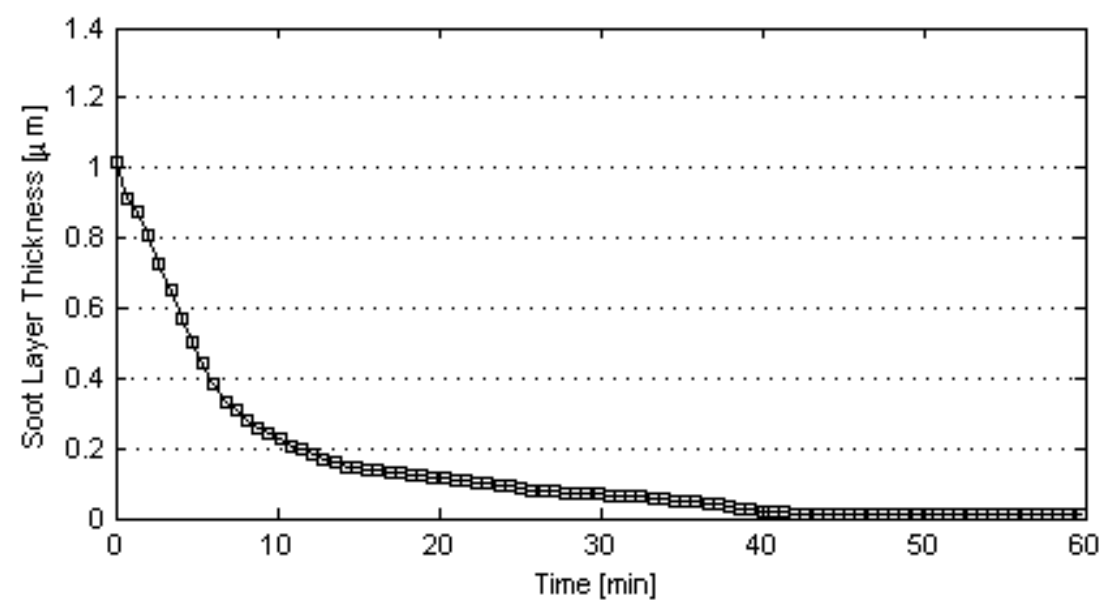

(a) Soot layer thickness.

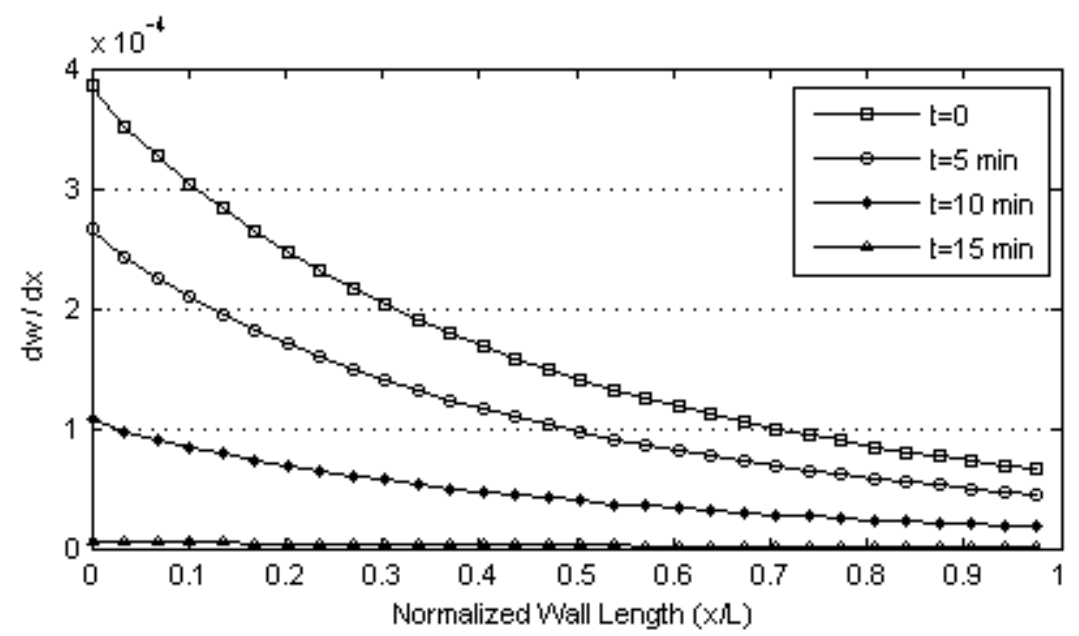

(b) Virtual soot layer per unit length.

Figure 6.37: Evolution of DPF properties during the regeneration process.

On the other hand, wall behavior over time for the R100 mode is completely different and represented by the soot layer per unit wall thickness. This variable is very useful to treat soot oxidation in the substrate wall and in the cake layer using a unified approach, allowing the use of the same constants and reaction rate definitions as discussed in Chapter 3. The value of $d w / d x$ in the washcoat/cake layer is always equal to unity, as it is the derivative of the thickness with respect to itself. On the other hand, since 
the wall obeys to different particle deposition rules, $d w / d x$ achieves a totally different order of magnitude and becomes a function of both time and wall thickness due to the differential soot loading across the thickness of the wall itself. Further, it is worth noting that the wall reaches an equilibrium condition in a shorter time, according to a more efficient oxidation process taking place in the wall rather than in the soot layer. Finally, results in terms of filter differential pressure are provided in Figure 6.38.
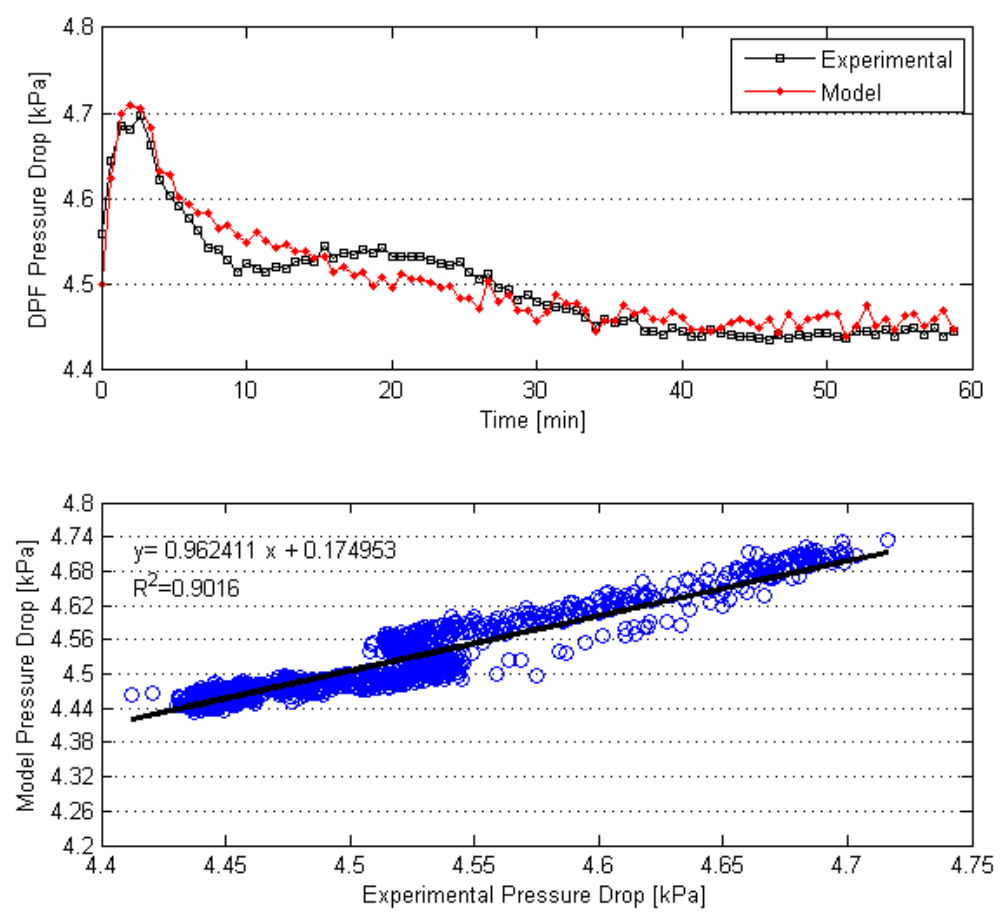

Figure 6.38: DPF differential pressure: simulated versus measured values.

Analysis of the results showed that the DOC-DPF model is capable of capturing the main phenomena taking place within both the DOC and DPF during a regeneration cycle. At first, the correct amount of $\mathrm{NO}$ and $\mathrm{NO}_{2}$ is computed by the DOC model together with the exhaust temperature. These signals are then provided to the DPF model which estimates the oxidation rate of PM mass and provides a simulated signal for the filter pressure drop. Finally, soot oxidation during the regeneration cycle is represented by means of the unified regeneration theory showing the link between oxidation of PM mass in the soot layer and substrate wall. 


\section{Chapter 7}

\section{Conclusions and Future Work}

\subsection{Conclusions}

This work reported the development and the calibration of a computational 1-D model for a diesel oxidation catalyst and a diesel particulate filter. The work was intended to provide a reliable model to be used for calibration and development of on-board diagnostics monitors. To this aim, the requirement that needed to be satisfied was the achievement of satisfactory accuracy without intensive computational effort and the capability to replicate experimentally measured values over a FTP transient engine cycle. Hence, the implementation of both DOC and DPF was aimed at achieving these goals.

For the purpose of generating a reliable DOC model, the following tasks have been accomplished:

- At first, a standard methodology was applied to generate a 1-D DOC model based on findings available in the open literature. This model is characterized by the solution of a set of non-linear algebraic equations involving surface concentrations, a set of differential equations for the computation of species concentrations in the bulk flow as a function of DOC axial position, and a set of differential equations representing the energy balance of the DOC.

- An innovative approach based on the definition of two limiting cases was then pro- 
posed to improve the efficiency of the computation. The resulting solution methodology neglects the estimation of the instantaneous concentration at the surface of the washcoat and implements two distinct solutions depending on the particular operating regime (kinetic or mass transfer limited).

- The need to improve model efficiency led also to the definition of a unique implementation strategy based on modular programming. The result of this approach is the DOC "Unit Cell" which contains all the correlations needed to simulate a virtual portion of the DOC. Further, an original implementation of the energy equation for the substrate wall aimed at including the effects of heat conduction was presented and compared to a classic methodology based on the utilization of Crank-Nicholson scheme.

- A calibration method based on the minimization of an objective function via the Levenberg-Marquardt algorithm was proposed and implemented to tune mass transfer parameters, frequency factors, and activation energies for the chemical reactions of interest.

For the purpose of generating a reliable DPF model, the following tasks have been achieved:

- A theoretical based, 1-D model over the wall thickness was generated and implemented to follow the evolution of a catalyzed diesel particulate filter.

- In order to lower the computational effort required by the model, an innovative approach based on analytical solution of the equations of interest was developed and used to track soot mass balance in the substrate wall.

- The resulting continuous wall filtration theory was coupled to an advanced treatment for the oxidation of soot in the wall. This led to the definition of a new variable, the virtual soot layer per unit wall thickness, which allowed for the representation of two regeneration dynamics utilizing a common theoretical treatment. 
- A new model calibration methodology called "Virtual Conditioning" was defined and applied to generate a robust set of tuning parameters which could be held constant during steady-state and transient operating conditions.

Validation of the models was performed by comparison of the simulated values with data gathered experimentally by coupling a Johnson Matthey C-CRT system with a MY2004 Mack MP7 heavy-duty diesel engine. The latter was exercised over a combination of steady-state and transient engine cycles to replicate loading and regeneration of the diesel particulate filter. According to the results obtained from the DOC model, the following conclusions could be drawn:

- The calibration methodology based on the minimization of the sum of squares of the error generated a set of apparent parameters which are in the ranges reported in previous studies (frequency factors, activation energies).

- The complete DOC model was capable of replicating experimental results in terms of DOC outlet concentrations, cumulative mass emissions and exhaust temperature during both steady-state and transient operation. The accuracy of the model to provide continuous traces for the DOC outlet concentrations and temperature over a full FTP cycle appeared to be in satisfactory.

- Although results in terms of nitric oxide emissions appeared to experience a deviation of less than 10\% from measured values, results for hydrocarbons and monoxide emissions presented larger discrepancies. This fact was attributed to the poor HC speciation used in this work, which led to representation of total hydrocarbons as a pure $C_{3} H_{6}$, and to the very low $C O$ concentrations measured downstream the DOC.

- The simplified model captured the main phenomena taking place within the diesel oxidation catalyst offering a much faster computation when compared to the complete model. Results indicate that the model based on limiting cases was able to replicate experimental results with an accuracy comparable to that of the complete 
model, requiring less than $1 / 3$ of the computational time.

- Oxidation of nitric oxide within the DOC appeared to be limited by thermodynamic equilibrium during the LAFY portion of the FTP cycle and during the regeneration mode due to the high temperature of the exhaust.

Discussion of the results generated by the DPF model led to the following conclusions:

- The model was capable of replicating the physical conditions of an aged DPF, resulting from subsequent loading/regeneration cycles. This was possible thanks to the improved filtration/regeneration theory which links deep bed filtration to the wall percolation factor and the filtration efficiency of the soot layer.

- The tuning methodology based on virtual conditioning generated a model calibration which resulted to be independent from the particular engine operating condition. The DPF model presented in this study has been tested holding constant calibration parameters such as activation energies, soot packing densities, and wall percolation factor. These parameters are usually adjusted manually depending on the particular engine mode whilst in this study no change was needed thanks to the tuning procedure based on virtual conditioning.

- The improved robustness of the model allowed for replication of a combination of engine cycles with a total duration of almost $30 \mathrm{hrs}$. Comparison of measured and simulated values showed that the calibrated DPF model is able to track filter soot loading with an accuracy of $\pm 5 g$, and filter pressure drop with an accuracy of $\pm 20 P a$. The capability to track filter properties over extended periods of operation suggests that the DPF model could be employed for OBD purposes.

- The substrate wall shows totally different regeneration and collecting dynamics than the washcoat/cake layers. This behavior is primarily important to capture backpressure and collected mass evolution over time.

- Analysis of the evolution of DPF properties during conditioning of the model showed that minimal amount of PM mass can produce significant changes in filter perme- 
ability with considerable effects on the overall pressure drop. In this regard, detailed simulation of the dynamics taking place in the wall is of primary importance also in case of aged/conditioned filter since the contribution of the wall accounts for more than $80 \%$ of the total pressure drop.

\section{2 $\quad$ Future Work}

The current work could be used as a framework for all the following future developments:

- The implementation strategy applied in this study could be utilized to generate a model for the Selective Catalytic Reduction (SCR) $N O_{x}$ abatement system. Further, the capabilities of the DOC model to estimate the transient $N O / N O_{x}$ ratio could be used to investigate the response of the SCR system in case of not-optimal feed-gas composition.

- The simulated engine exhaust temperature at the outlet of the diesel oxidation catalyst could be utilized to develop OBD monitors for the detection of missing substrate based on the time shift resulting from the thermal inertia.

- The DPF model could be utilized to define maps for the filter pressure drop as function of exhaust temperature and flow rate, for both a fully loaded and a clean filter. A soot loading monitor could then be developed using the filter pressure drop signal as input and interpolating between the two limiting cases to estimate the soot content of filter. 


\section{References}

[1] S.R. Katare, J.E. Patterson, and P.M. Laing. Diesel aftertreatment modeling: a systems approach to nox control. Industrial Engineering Chemistry Product Research and Development, 46:2445-2454, 2007.

[2] G. Corro. Sulfur impact on diesel emission control. Reaction Kinetics and Catalysis Letters, 75:89-106, 2002.

[3] W. Pulkrabek. Engineering Fundamentals of the Internal Combustion Engine. Pearson Prentice Hall, 2004.

[4] D. Iverach, K.S. Basden, and N.Y. Kirov. Formation of nitric oxide in fuel-lean and fuel-rich flames. Symposium on Combustion, 14:767-775, 1973.

[5] Y. Kitamura, A. Mohammadi, T. Ishiyama, and M. Shioji. Fundamental investigation of $\mathrm{NO}_{x}$ formation in diesel combustion under supercharged and EGR conditions. SAE Technical Paper 2005-01-0364, 2005.

[6] J.B. Heywood. Internal Combustion Engine Fundamentals. McGraw Hill, Inc., 1988.

[7] A. Cozzolini, M.C. Besch, R. Ardanese, M. Ardanese, and M. Gautam. Determination of optimal engine parameters for exhaust emissions reduction using the Taguchi method. ASME ICEF2011-60134, 2011.

[8] M. Ardanese, R. Ardanese, T. Adams, M.C. Besch, V. Sathi, B. Shade, and M. Gautam. Development of ADECS to meet 2010 emission levels: optimization of $\mathrm{NO}_{x}$, $\mathrm{NH}_{3}$ and fuel consumption using high and low engine-out $\mathrm{NO}_{x}$ calibrations. 14th DEER Conference, 2008.

[9] M. Yamamoto, S. Yoneya, T. Matsuguchi, and Y. Kumagai. Optimization of heavyduty diesel engine parameter for low exhaust emissions using the design of experiments. SAE Technical Paper 2002-01-1148, 2002.

[10] U.S. Environmental Protection Agency. U.S. EPA Air quality criteria for ozone and related photochemical oxidants. Report EPA600/R-05/004aF, 2006. 
[11] M.L. Bell, A. McDermott, S.L. Zeger, J.M. Samet, and F. Dominici. Ozone and short-term mortality in 95 US urban communities, 1987-2000. Journal of the American Medical Association, 292:2372-2378, 2004.

[12] M.S. Goldberg, R.T. Burnett, J. Brook, J.C. Bailar III, M.F. Valois, and R. Vincent. Associations between daily cause-specific mortality and concentrations of groundlevel ozone in Montreal, Quebec. American Journal of Epidemiology, 154:817-826, 2000.

[13] J.A. Bernstein, N. Alexis, C. Barnes, I.L. Bernstein, A. Nel, D. Peden, D. DiazSanchez, S. Tarlo, and P.B. Williams. Health effects of air pollution. The Journal of Allergy and Clinical Immunology, 114:1116-1123, 2004.

[14] T. Kamimoto and M. Bae. High combustion temperature for the reduction of particulate in diesel engines. SAE Technical Paper 880423, 1988.

[15] M.V. Twigg and P.R. Phillips. Cleaning the air we breathe - controlling diesel particulate emissions from passenger cars. Platinum Metals Review, 53:27-34, 2009.

[16] D.B. Kittelson, W.F. Watts, and M. Arnold. Review of diesel particulate matter sampling methods. Final Report, University of Minnesota, Center for Diesel Research, Minneapolis, MN., 1998.

[17] B. Giechaskiel, L. Ntziachristos, Z. Samaras, V. Scheer, R. Casati, and R. Vogt. Formation potential of vehicle exhaust nucleation mode particles on-road and in the laboratory. Atmospheric Environment, 39:3191-3198, 2005.

[18] C.A. Pope. Epidemiology of fine particulate air pollution and human health: biologic mechanisms and who's at risk. Environmental Health Perspectives, 108:713723,2000 .

[19] G. Oberdorster, J. Ferin, S. Soderholm, R. Gelein, C. Cox, R. Baggs, and P.E. Morrow. Increased pulmonary toxicity of inhaled ultrafine particles: due to lung overload alone? Annals of Occupational Hygiene, 38:295-302, 1994.

[20] Z.D. Ristovski, B. Miljevic, N.C. Surawski, K.M. Fong, and I.A. Yang. Respiratory health effects of diesel particulate matter. Respirology, 17:201-212, 2012.

[21] DHHS (NIOSH). Carcinogenic effects of exposure to diesel exhaust. Current Intelligence Bulletin 50, Publication Number 88-116, 1988.

[22] U.S. Environmental Protection Agency. Heavy-Duty Highway CompressionIgnition Engines and Urban Buses: Exhaust Emission Standards. Emission Standards Reference Guide, http://www.epa.gov, 2011. 
[23] California Air Resources Board. California Code of Regulations, Section 1971.1, Title 13. http://www.oal.ca.gov/, 2013.

[24] T. Johnson. Diesel engine emissions and their control. Platinum Metals Review, $52: 23-37,2008$.

[25] L.M. Pickett. Soot formation at low flame temperature diesel operating conditions. 9th International Conference "Present and Future Engines for Automobiles", 2005.

[26] P. Adomeit. Laser optical diagnostics and numerical analysis of hsdi combustion systems. THIESEL 2004, 2004.

[27] T. Johnson. Vehicular emissions in review. SAE Technical Paper 2013-01-0538, 2013.

[28] I. Gruden. The new ice age. DEER Conference 2012, 2012.

[29] T.J. Jacobs. Simultaneous reduction in NOx and particulate matter emissions from a ligth-duty diesel engine using combustion development and diesel oxidation catalyst. Ph.D. Thesis, University of Michigan, 2005.

[30] K. Okude, K. Mori, S. Shiino, and T. Moriya. Premixed compression ignition (pci) combustion for simultaneous reduction of $\mathrm{NO}_{x}$ and soot in diesel engines. SAE Technical Paper 2004-01-1907, 2004.

[31] M. Ardanese. Simple strategy-based technique to reduce emissions from SCR-equipped heavy-duty diesel engine over different engine-out calibrations. Ph.D. Thesis, West Virginia University, 2008.

[32] R. Ardanese, M. Ardanese, M. Besch, and T. Adams. Pm concentration and size distributions from a heavy-duty diesel engine programmed with different engine-out calibrations to meet the 2010 emission limits. SAE Technical Paper 2009-01-1183, 2009.

[33] F. Haas and H. Fuess. Structural characterization of automotive catalysts. Advanced Engineering Materials, 7:899-913, 2005.

[34] R.J. Farrauto. New applications of monolithic supported catalysts. Reaction Kinetics and Catalysis Letters, 60:233-241, 1997.

[35] Z. Liu and S.I. Woo. Recent advances in catalytic de- $\mathrm{NO}_{x}$ science and technology. Catalysis Reviews, 48:43-89, 2006.

[36] L. Olsson and E. Fridell. The influence of pt oxide formation and pt dispersion on the reactions $\mathrm{NO}_{2}<->\mathrm{NO}+1 / 2 \mathrm{O}_{2}$ over $\mathrm{Pt} / \mathrm{Al}_{2} \mathrm{O}_{3}$ and $\mathrm{Pt} / \mathrm{BaO} / \mathrm{Al}_{2} \mathrm{O}_{3}$. Journal of Catalysis, 210:340-353, 2002. 
[37] R. Burch, P.J. Millington, and A.P. Walker. Mechanism of the selective reduction of nitrogen monoxide on platinum-based catalysts in the presence of excess oxygen. Applied Catalysis B: Environmental, 4:65-94, 1994.

[38] W. Held, A. Kaonig, T. Richter, and L. Puppe. Catalytic $\mathrm{NO}_{x}$ reduction in net oxidizing exhaust gas. SAE Technical Paper 900496, 1990.

[39] C.S. Kang, Y.J. You, K.J. Kim, T.H. Kim, S.J. Ahn, K.H. Chung, N.C. Park, S. Kimura, and H.G. Ahn. Selective catalytic reduction of NOx with propene over double wash-coat monolith catalysts. Catalysis Today, 111:229-235, 2006.

[40] D. Chatterjee, T. Burkhardt, T. Rappe, A. Guthenke, and M. Weibel. Numerical simulation of DOC $+\mathrm{DPF}+\mathrm{SCR}$ systems: DOC influence on SCR performance. SAE Technical Paper 2008-01-0867, 2008.

[41] M. Li, J. Henao, Y. Yeom, E. Weitz, and W. Sachtler. Low activation energy pathway for the catalyzed reduction of nitrogen oxides to $\mathrm{N}_{2}$ and ammonia. Catalysis Letters, 98:5-9, 2004.

[42] M. Colombo, I. Nova, E. Tronconi, D. Chatterjee, and M. Weibel. A comparative study of the $\mathrm{NH}_{3}$-SCR reactions over a Cu-zeolite and Fe-zeolite catalyst. Catalysis Today, 151:223-230, 2010.

[43] A. Grossale, I. Nova, E. Tronconi, D. Chatterjee, and M. Weibel. $\mathrm{NH}_{3}-\mathrm{NO} / \mathrm{NO}_{2}$ SCR for diesel exhausts aftertreatment: reactivity, mechanism and kinetic modeling of commercial Fe- and Cu-promoted zeolite catalysts. Topics in Catalysis, 52:18371841, 2009.

[44] I. Nova, C. Ciardelli, E. Tronconi, D. Chatterjee, and B. Bandl-Konrad. $\mathrm{NH}_{3}{ }^{-}$ $\mathrm{NO} / \mathrm{NO}_{2}$ chemistry over V-based catalysts and its role in the mechanism of the fast SCR reaction. Catalysis Today, 114:3-12, 2006.

[45] S.R. Katare, J.E. Patterson, and P.M. Laing. Aged doc is a net consumer of $\mathrm{NO}_{2}$ : analyses of vehicle, engine-dynamometer and reactor data. SAE Technical Paper 2007-01-3984, 2007.

[46] W.A. Majewski and M.K. Khair. Diesel emissions and their control. SAE International: Warrendale, PA, 2006.

[47] M. Koebel, G. Madia, and M. Elsener. Selective catalytic reduction of $\mathrm{NO}$ and $\mathrm{NO}_{2}$ at low temperatures. Catalysis Today, 73:239-247, 2002.

[48] J. Despres, M. Elsener, M. Koebel, O. Krocher, B. Schnyder, and A. Wokaun. Catalytic oxidation of nitrogen monoxide over Pt/SiO${ }_{2}$. Applied Catalysis B: Environmental, 50:73-82, 2002. 
[49] M. Shost, J. Noetzel, M. Wu, T. Sugiarto, T. Bordewyk, G. Fulks, and B. Fisher. Monitoring, feedback and control of urea SCR dosing systems for $\mathrm{NO}_{x}$ reduction: utilizing an embedded model and ammonia sensing. SAE Technical Paper 2008-01$1325,2008$.

[50] C.M. Schar, C.H. Onder, and H.P. Geering. Control-oriented model of an SCR catalytic converter system. SAE Technical Paper 2004-01-0153, 2004.

[51] C.Y. Ong and A.M. Annaswamy. An adaptive proportional integral control of a urea selective catalytic reduction system based on system identification models. SAE Technical Paper 2010-01-1174, 2010.

[52] T. Johnson. Diesel emission control in review. SAE International Journal of Fuels and Lubricants, 2, 2009.

[53] M. Twigg. Roles of catalytic oxidation in control of vehicle exhaust emissions. Catalysis Today, 117:407-418, 2006.

[54] C. Tien. Granular Filtration of Aerosols and Hydrosols. Butterworths, 1989.

[55] P. Abeyratne. A two dimensional numerical soot model for advanced design and control of diesel particulate filter. Master Thesis, West Virginia University, 2011.

[56] D. Rose and T. Boger. Different approaches to soot estimation as key requirement for DPF applications. SAE Technical Paper 2009-01-1262, 2009.

[57] A.G. Konstandopoulous, M. Kostoglou, E. Skaperdas, E. Papaioannou, D. Zarvalis, and E. Kladopoulou. Fundamental studies of diesel particulate filters: transient loading, regeneration and aging. SAE Technical Paper 2000-01-1016, 2000.

[58] C.N. Opris and J.H. Johnson. A 2-D computational model describing the flow and filtration characteristics of a ceramic diesel particulate trap. SAE Technical Paper 980545, 1998.

[59] C.N. Davis. Air Filtration. Academic Press Inc., 1973.

[60] E. Xue, K. Seshan, and J.R.H. Ross. Roles of supports, Pt loading and Pt dispersion in the oxidation of $\mathrm{NO}$ to $\mathrm{NO}_{2}$ and of $\mathrm{SO}_{2}$ to $\mathrm{SO}_{3}$. Applied Catalysis B: Environmental, 11:65-79, 1996.

[61] J. Jung, S. Song, and K.M. Chun. Characterization of catalyzed soot oxidation with $\mathrm{NO}_{2}$, NO and $\mathrm{O}_{2}$ using a lab-scale flow reactor system. SAE Technical Paper 2008-01-0482, 2008. 
[62] E.A. Kladopoulou, S.L. Yang, J.H. Johnson, G.G. Parker, and A.G. Konstandopoulos. A study describing the performance of diesel particulate filters during loading and regeneration. SAE Technical Paper 2003-01-0842, 2003.

[63] V. Mulone, A. Cozzolini, P. Abeyratne, D. Littera, M. Thiagaraian, M.C. Besch, and M. Gautam. Soot modeling for advanced control of diesel engine aftertreatment. ASME Journal of Engineering for Gas Turbine, Engine and Power, 133:122804$122816,2010$.

[64] K. Bencherif, F. Benaicha, S. Sada, and M. Sorine. Diesel particulate filter thermal management using model-based design. SAE Technical Paper 2009-01-1082, 2009.

[65] G. Mauviot, F. Le Berr, S. Raux, F. Perretti, L.M. Malbec, and C.N. Millet. 0D modelling: a promising means for after-treatment issues in modern automotive applications. Oil \& Gas Science and Technology, 64:285-307, 2009.

[66] A. Mayer, T. Lutz, C. Lammle, and M. Wyser. Engine intake throttling for active regeneration of diesel particle filters. SAE Technical Paper 2003-01-0381, 2003.

[67] M. Khair. A review of diesel particulate filter technologies. SAE Technical Paper 2003-01-2303, 2003.

[68] A. Setiabudi, M. Makkee, and J.A. Moulijn. The role of $\mathrm{NO}_{2}$ and $\mathrm{O}_{2}$ in the accelerated combustion of soot in diesel exhaust gases. Applied Catalysis B: Environmental, 50:185-194, 2004.

[69] S. Banerjee. Thermal analysis of active catalytic diesel particulate filter regeneration. Master Thesis, University of Windsor, 2007.

[70] R. Isermann and P. Balle. Trends in the application of model-based fault detection and diagnosis of technical processes. Control Engineering Practice, 5:709-719, 1997.

[71] R.M. Heck and R.J. Farrauto. Catalytic Air Pollution Control. Van Nostrand Reinhold: New York, 1995.

[72] P.G. Blakeman, A.F. Chiffey, P.R. Phillips, M.V. Twigg, and A.P. Walker. Developments in diesel emission aftertreatment technology. SAE Technical Paper 2003-01-3753, 2003.

[73] A. Russell and W. Epling. Diesel oxidation catalysts. Catalysis Reviews: Science and Engineering, 53:337-423, 2011.

[74] U. Lassi. Deactivation correlations of $\mathrm{Pd} / \mathrm{Rh}$ three-way catalysts designed for EuroIV emission limits: effect of ageing atmosphere, temperature and time. Oulu University Press, 2003. 
[75] M. Kamijo, M. Kamikubo, H. Akama, and K. Matsushita. Study of an oxidation catalyst system for diesel emission control utilizing $\mathrm{HC}$ adsorption. JSAE Rewview, $22: 277-280,2001$.

[76] K.M. Adams, J.V. Cavataio, T. Sale, W.A. Rimkus, and R.H. Hammerle. Laboratory screening of diesel oxidation catalysts and validation with vehicle testing: the importance of hydrocarbon storage. SAE Technical Paper 962049, 1996.

[77] A. Blackwood, D. Tidmarsh, and M. Willcock. The effect of an oxidation catalyst on cold start diesel emissiosn in the first 120 seconds of running. SAE Technical Paper 980193, 1998.

[78] R.J. Farrauto and K.E. Voss. Monolith diesel oxidation catalysts. Applied Catalysis B: Environmental, 10:29-51, 1996.

[79] S. Verdier. Innovative materials for diesel oxidation catalysts with high durability and early light-off. SAE Technical Paper 2005-01-0476, 2005.

[80] K. Tashiro, S. Ito, A. Oba, and T. Yokomizo. Development of oxidation catalyst for diesel passenger car. JSAE Review, 16:131-136, 1995.

[81] H.C. Yao. Ceria in automotive exhaust catalysts. Journal of Catalysis, 86:254-265, 1984.

[82] S. Cordiner, F. Mecocci, V. Mulone, and M. Nobile. Model based design procedure of after treatment systems for non-road diesel engines. SAE Technical Paper 201124-0186, 2011.

[83] G.C. Koltsakis, P.A. Konstantinidis, and A.M. Stamatelos. Development and application range of mathematical models for 3-way catalytic converters. Applied Catalysis B: Environmental, 12:161-191, 1997.

[84] C.S. Sampara, E.J. Bissett, M. Chmielewski, and D. Assanis. Global kinetics for platinum diesel oxidation catalysts. Applied Catalysis B: Environmental, 46:79938003, 2007.

[85] H. Santos and M. Costa. Analysis of the mass transfer controlled regime in automotive catalytic converters. International Journal of Heat and Mass Transfer, 51:41-51, 2008.

[86] C.S. Sampara. Global reaction kinetics for oxidation and storage in diesel oxidation catalysts.. Ph.D. Thesis, University of Michigan, 2008.

[87] A.K. Neyestanaki, F. Klingstedt, T. Salmi, and D.Y. Murzin. Deactivation of postcombustion catalysts: a review. Fuel, 83:395-408, 2004. 
[88] C.P. Hubbard, K. Otto, H.S. Gandhi, and K.Y.S. Ng. Propane oxidation over platinum supported on zirconia. Journal of Catalysis, 139:268-276, 1993.

[89] S. Cordiner, G. De Simone, and V. Mulone. Influence of washcoat distribution on the performance of diesel oxidation catalysts. SAE Technical Paper 2007-01-4007, 2007.

[90] V. Balakotaiah, N. Gupta, and D. West. A simplified model for analyzing catalytic reactions in short monoliths. Chemical Engineering Science, 55:5367-5383, 2000.

[91] R.E. Hayes and S.T. Kolaczkowski. Mass and heat transfer effects in catalytic monolith reactors. Chemical Engineering Science, 49:3587-3599, 1994.

[92] F. Lafossas, Y. Matsuda, and A. Mohammadi. Calibration and validation of a diesel oxidation catalyst model: from synthetic gas testing to driving cycle applications. SAE Technical Paper 2011-01-1244, 2011.

[93] J. Desprs, M. Elsener, M. Koebel, O. Krcher, B. Schnyder, and A. Wokaun. Catalytic oxidation of nitrogen monoxide over $\mathrm{Pt} / \mathrm{SiO}_{2}$. Applied Catalysis B: Environmental, 50:73-82, 2004.

[94] C.N. Millet and S. Benramdhane. A 3WCC global kinetic model: a calibration method using laboratory scale and engine test bench experiments. SAE Technical Paper 2008-01-0453, 2008.

[95] G.N. Pontikakis, G.C. Koltsakis, A.M. Stamatelos, R. Noirot, Y. Agliany, H. Colas, P. Versaevel, and C. Bourgeois. Experimental and modeling study on zeolite catalysts for diesel engines. Topics in Catalysis, 16-17:329-335, 2001.

[96] T. Shamim, H. Shen, S. Sengupta, S. Son, and A.A. Adamczyk. A comprehensive model to predict three way catalytic converter performance. Journal of Engineering for Gae Turbines and Power, Transactions, 124:329-335, 2002.

[97] G.C. Koltsakis, I.P. Kandylas, and A.M. Stamatelos. Three way catalytic converter modeling and applications. Chemical Engineering Communications, 164:153-189, 1998.

[98] C. Huynh, J. Johnson, S. Yang, and S. Bagley. A one-dimensional computational model for studying the filtration and regeneration characteristics of a catalyzed wall-flow diesel particulate filter. SAE Technical Paper 2003-01-0841, 2003.

[99] A. Konstandopoulos, E. Skaperdas, J. Warren, and R. Allansson. Optimized filter design and selection criteria for continuously regenerating diesel particulate traps. SAE Technical Paper 1999-01-0468, 1999. 
[100] H. Aoki, A. Asano, K. Kurazono, K. Kobashi, and H. Sami. Numerical simulation model for the regeneration process of a wall-flow monolith particulate filter. SAE Technical Paper 930364, 1993.

[101] K. Kuo. Principles of Combustion. John Wiley \& Sons, New York, 1986.

[102] S. Cordiner, G. DeSimone, and V. Mulone. A general 3-D model to analyze particle transport into a partial-flow particulate filter. SAE International Journal of Fuels and Lubricants, 3:452-467, 2010.

[103] S. Cordiner and V. Mulone. 3-D unsteady modeling of the loading process in a diesel engine PM-filter. SAE Technical Paper 2007-01-1132, 2007.

[104] M. Masoudi, A. Heibel, and P. Then. Predicting pressure drop of wall-flow diesel particulate filters - theory and experiment. SAE Technical Paper 2000-01-0184, 2000.

[105] A. Konstandopoulos, E. Skaperdas, and M. Masoudi. Inertial contributions to the pressure drop of diesel particulate filters. SAE Technical Paper 2001-01-0909, 2001.

[106] A.G. Konstandopoulos and M. Kostoglou. Periodically reversed flow regeneration of diesel particulate traps. SAE Technical Paper 1999-01-0469, 1999.

[107] A.G. Konstandopoulos and M. Kostoglou. Reciprocating flow regeneration of soot filters. Combustion and Flame, 121:488-500, 2000.

[108] E.J. Bisset. Mathematical model of the thermal regeneration of a wall-flow monolith diesel particulate filter. Chemical Engineering Science, 39:1233-1244, 1984.

[109] K.W. Lee and J.A. Gieseke. Collection of aerosol particles by packed beds. Environmental Science Technology, 13:466-470, 1978.

[110] S. Oh, J. McDonald, G. Vaneman, and L. Hegedus. Mathematical modeling of fibrous filters for diesel particulate-theory and experiments. SAE Technical Paper 810113, 1981.

[111] A.G. Konstandopolous and J.H. Johnson. Wall-flow diesel particulate filters: their pressure drop and collection efficiency. SAE Technical Paper 890405, 1989.

[112] A. Konstandopoulos and M. Kostoglou. Microstructural aspects of soot oxidation in diesel particulate filters. SAE Technical Paper 2004-01-0693, 2004.

[113] N. Singh, C. Rutland, D. Foster, and K. Narayanaswamy. Investigation into different DPF regeneration strategies based on fuel economy using integrated system simulation. SAE Technical Paper 2009-01-1275, 2009. 
[114] M. Schejbal, M. Marek, M. Kubicek, and P Koci. Modelling of diesel filters for particulates removal. Chemical Engineering Journal, 154:219-230, 2009.

[115] S. Charbonnel and C. Opris. Fundamental diesel particulate filter (DPF) pressure drop model. SAE Technical Paper 2009-01-1271, 2009.

[116] N. Nagar, X. He, V. Iyengar, and N. Acharya. Real time implementation of DOCDPF models on a production-intent ECU for controls and diagnostics of a PM emission control system. SAE International Journal of Commercial Vehicles, 2:222233, 2009.

[117] S.E. Voltz, C.R. Morgan, D. Liederman, and S.M. Jacob. Kinetic study of carbon monoxide and propylene oxidation on platinum catalysts. Industrial Engineering Chemistry Product Research and Development, 12:294-301, 1973.

[118] J.C. Cavendish and S.H. Oh. A computationally efficient Galerkin technique for approximating transient diffusion-reaction equations in composite media. The Chemical Engineering Journal, 17:41-54, 1979.

[119] S.H. Oh and J.C. Cavendish. Transients of monolithic catalytic converters: response to step changes in feedstream temperature as related to controlling automobile emissions. Industrial Engineering Chemistry Product Research and Development, 21:29-37, 1982.

[120] J.C. Schlatter and T.S. Chou. Measuring oxidation rates in a recycle reactor. AIChE 71st Annual Meeting, 1978.

[121] A.P. Triana, J.H. Johnson, S.L. Yang, and K.J. Baumgard. An experimental and numerical study of the performance characteristics of a diesel oxidation catalyst in a continuously regenerating particulate filter. SAE Technical Paper 2003-01-3176, 2003.

[122] I.P. Kandylas, O.A. Haralampous, and G.C. Koltsakis. Diesel soot oxidation with $\mathrm{NO}_{2}$ : engine experiments and simulations. Industrial Engineering and Chemistry Research, 41:5372-5384, 2002.

[123] G.C. Koltsakis, O.A. Haralampous, C.K. Dardiotis, and Z.C. Samaras. Performance of catalyzed particulate filters without upstream oxidation catalyst. SAE Technical Paper 2005-01-0952, 2005.

[124] A.P.E. York, J.P. Cox, T.C. Watling, A.P. Walker, and D. Bergeal. Development and validation of a one-dimensional computational model of the continuously regenerating diesel particulate filter (CR-DPF) system. SAE Technical Paper 200501-0954, 2005. 
[125] G. Groppi, A. Belloli, E. Tronconi, and P. Forzatti. A comparison of lumped and distributed models of monolith catalytic combustors. Chemical Engineering Science, 50:2705-2715, 1995.

[126] H. Sharma and A. Mhadeshwar. A detailed microkinetic model for diesel engine emissions oxidation on platinum based diesel oxidation catalyst (DOC). Applied Catalysis B: Environmental, 127:190-204, 2012.

[127] O.A. Haralampous, G.C. Koltsakis, and Z.C. Samaras. Reaction and diffusion phenomena in catalyzed diesel particulate filters. SAE Technical Paper 2004-010696, 2004.

[128] T. Shamim. Effect of heat and mass transfer coefficients on the performance of automotive catalytic converters. International Journal of Engine Research, 4:129$141,2002$.

[129] S.H. Chan and D.L. Hoang. Heat transfer and chemical reactions in exhaust system of a cold-start engine. International Journal of Heat and Mass Transfer, 42:41654183, 1999.

[130] K.C. Premchand, J.H. Johnson, S.L. Yang, A.P. Triana, and K.J. Baumgard. A study of filtration and oxidation characteristics of a diesel oxidation catalyst and a catalyzed particulate filter. SAE Technical Paper 2007-01-1123, 2007.

[131] E.J. Bisset and F. Shadman. Thermal regeneration of diesel particulate monolithic filters. A.I.Ch.E. Journal, 39:753-758, 1985.

[132] A. Cozzolini, V. Mulone, P. Abeyratne, D. Littera, and M. Gautam. Advanced modeling of diesel particulate filters to predict soot accumulation and pressure drop. SAE Technical Paper 2011-24-0187, 2011.

[133] I.P. Kandylas and G.C. Koltsakis. $\mathrm{NO}_{2}$ assisted regeneration of diesel particulate filters: a modeling study. Industrial Engineering and Chemistry Research, 41:21152123, 2002.

[134] Di. Huang. Modeling of diesel particulate filter filtration and regeneration for transient driving schedules. Ph.D. Thesis, Michigan Technological University, 2011.

[135] H. Mohammed, A.P. Triana, S.L. Yang, and J.H. Johnson. An advanced 1-d 2layer catalyzed diesel particulate filter model to simulate: filtration by the wall and particulate cake, oxidation in the wall and particulate cake by $\mathrm{NO}_{2}$ and $\mathrm{O}_{2}$, and regeneration by heat addition. SAE Technical Paper 2006-01-0467, 2006. 
[136] A.G. Konstandopoulous and M. Kostoglou. Computationally fast implementations of convection, diffusion and chemical reaction phenomena in diesel particulate filters. SAE Technical Paper 2010-01-0890, 2010.

[137] I.P. Kandylas and G.C. Koltsakis. Simulation of continuously regenerating diesel particulate filters in transient driving cycles. Journal of Automotive Engineering, 216:591-606, 2002.

[138] A.P. Walker, R. Allanson, P.G. Blakeman, B.J. Cooper, H. Hess, and P.J. Silcock. Optimizing the low temperature performance and regeneration efficiency of the continuously regenerating diesel particulate filter (CR-CPF) system. SAE Technical Paper 2002-01-0072, 2002.

[139] A. Setiabudi, M. Makkee, and J.A. Moulijn. An optimal NO $\mathrm{N}_{x}$ assisted abatement of diesel soot in an advanced catalytic filter design. Applied Catalysis B: Environmental, 42:35-45, 2003.

[140] B.J. Cooper and J.E. Thoss. Role of NO in diesel particulate emission control. SAE Technical Paper 890404, 1989.

[141] A. Bejan. Convection Heat Transfer. John Wiley \& Sons, 2004.

[142] G.C. Koltsakis, C.K. Dardiotis, Z.C. Samaras, T. Maunula, T. Kinnune, and P. Lundorf. Optimization methodologies for DPF substrate-catalyst combinations. SAE Technical Paper 2009-01-0291, 2009.

[143] A. Heibel. Evaluation of the new Corning DuraTrap AT diesel particulate filter results from engine bench and vehicle tests. 14 Aachener Kolloquium Fahrzeug und Motorentechnik, 14:193-218, 2005.

[144] P.G. Eastwood, K. Tufail, T. Winstanley, A. Darlington, S. Karagiorgis, Y. Hardalupas, and A. Taylor. Estimations of deviations in NO and soot emissions between steady-state and EUDC transient operation of a common-rail diesel engine. SAE Technical Paper 2009-24-0147, 2009. 